\title{
INFRARED AND RAMAN SPECTRA \\ OF GASEOUS OXALIC ACID
}

by

\section{Chitti Oralratmanee}

A thesis sulmitted for the degree of Doctor of Philosophy of the iniversity of Surrey.

Dopartment of Chemical Physics,

March, 1973 University of Surrey 
ProQuest Number: 10804327

All rights reserved

INFORMATION TO ALL USERS

The quality of this reproduction is dependent upon the quality of the copy submitted.

In the unlikely event that the author did not send a complete manuscript and there are missing pages, these will be noted. Also, if material had to be removed, a note will indicate the deletion.

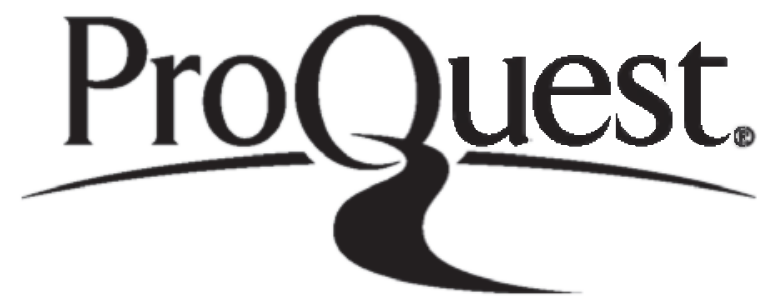

ProQuest 10804327

Published by ProQuest LLC (2018). Copyright of the Dissertation is held by the Author.

All rights reserved.

This work is protected against unauthorized copying under Title 17, United States Code Microform Edition (C) ProQuest LLC.

ProQuest LLC.

789 East Eisenhower Parkway

P.O. Box 1346

Ann Arbor, Ml $48106-1346$ 


\section{ACWNOHLDOCAMNTS}

I would like to thank supervisor, Dr. B. C. Stace, for giving we every assistance during the project and also for providing the necessary background knowledge that hes enabled ino to undertake this project.

A scholarship from the Colombo plan is gratefully acknonledged. 
SURMARY

The vibrational spectrun of gaseous oxalic acid was studied. Oxalic acid is a planar rolecule with $C_{2 h}$ symotry and 18 fundamentals. Tro structures based on clectron diffraction studios have previcusly boen proposed:

I

II
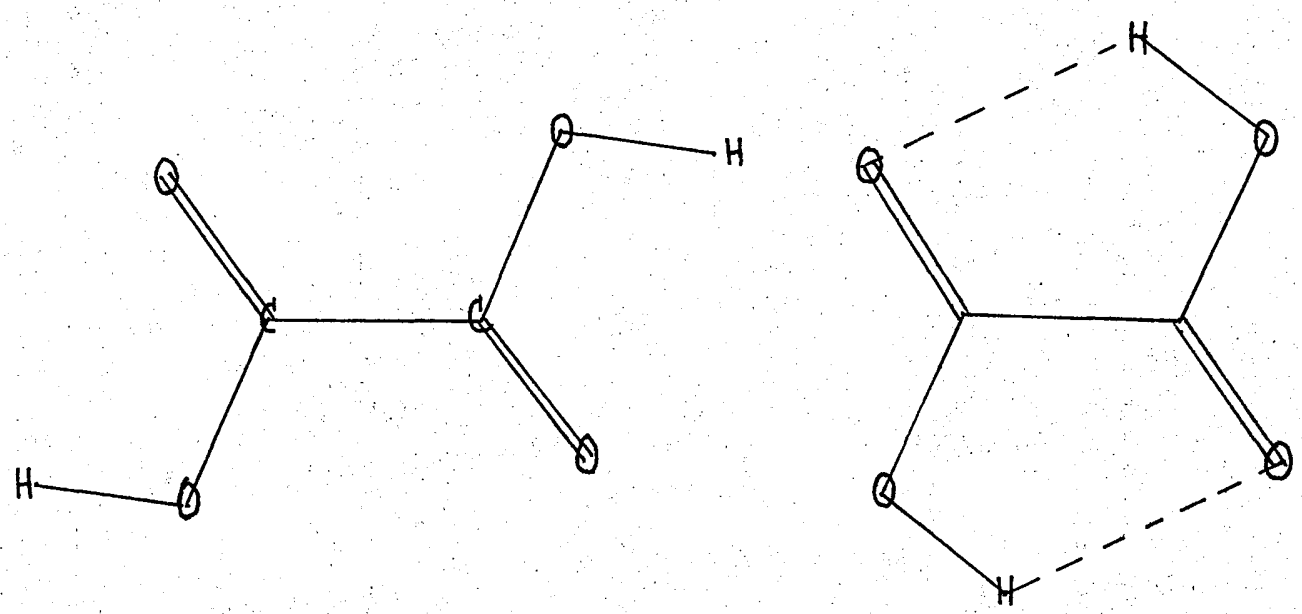

8 infrared baids of gaseous oxalic acid were obsorved in the frequency range $4000-350 \mathrm{~cm}^{-1}$. S were assigned to inplane funciamental modes, 2 were assigned to out of plone fundamental rodes, and only 1 was assigned to combination mode.

5 Raman bands of gaseous oxalic acid were observed and all were assigned to inplane fundegental modss.

Previously 6 infrared bands have been observed but no previous Raman study of tho vapour has been publisined.

A Urey-Bradiey force field (refined by trial and error) was used to calculate frequencies, potantial onergy distributions and normal rodes of vibration for both structures. The spectroscopic assignmonts and vibrational analysis are equally consistent with 
the two proposed structuros and therefore support a centrosyaretric structure.

The contours of the infrared bands do not help in assigaing the fundmentals because the inplane banis are $A-B$ hybrids. In addition the monts of incrtia of both structures are too close to be able to dicide betweon the two structures.

The infrared spectra were recorded with a $60 \mathrm{~cm}$ path gas coll and a Porkin-Elnar 457 spectroneter. Temporatures between $70^{\circ} \mathrm{C}$ and $125^{\circ} \mathrm{C}$ were used.

The Rama spectra ware recorded with a Spex 1401 spectroneter and approxinately 2 watts at $5145 \mathrm{~A}^{\circ}$ from a Spectra-physics 164 1asor. Temperatures between $140^{\circ} \mathrm{C}$ and $185^{\circ} \mathrm{C}$ were used. 


\section{CONTENTS}

Page

Introduction

Chapter 1: Hotion in Cartesian Co-ordinates

1.1 Quantun mechmical description on the basis of the harmonic oscillator model

2.2 Normal modes of vibration 3

1.3 Wotion in cartesian displacement co-ordinates 4

1.4 Hotion in nass weighted cartesian displacenent co-ordinates

1.5 Determination of tho form of the nomal wodes 8

Chapter 2: Motion in Intornal Co-ordinates

2.1 Kinetic energy in terms of internal co-ordinates

2.2 Potential energy in terns of intemai co-ordinates

2.3 The secular equation in terms of internal co-ordinates

Cnapter 3: Motion in Symatry Co-ordinates

3.1 Symetry co-ordinates $\quad 17$

3.2 Construction of symetry co-ordinates 20

Chepter 4: Hotion in Norral Co-ordinates

4.1 Ninetic and potential onergies in nomal co-ordinates:

4.2 Detemination of normal co-ordinates 23

4.3 Potential enorgy distribution 25

Chapter 5: G-Natrix

5.1 Construction of the G matrix 28

5.2 Evaluation of the 3 natrix 28 
Chapter 6: Force Fields

6.1 Force constants 33

6.2 Force fields 34

6.3 Urey-Bradley force field 36

Chapter 7: Force Constane Calculations

7.1 Nethod of trial and error 41

7.2 Method of least squares refinement 42

7.3 Nethod of obtaining the Jacobian matrix 45 for the least squares nethod

7.4 A probien in the least squares mathod 49

7.5 The uncertainties in tine caiculation so

Chapter 8: Infrared Transitions

8.1 Harmonic vibration selection rules 51

8.2 Anharaonicity $\quad 52$

Chapter 9: Ranan Transitions

9.1 Raman polarizability tensor 55

Q.2 Expansion of the polarizability in the
nomal co-ordinates

9.3 Degree of dopolarization $\quad 58$

9.4 Raman selection rules $\quad 60$

Chapter 10: Energy Lovels of Asymatric Top Mlecules

10.1 Rotational energy levels of asymetric top nolecules

10.2 Quantitative formulae for the energy levels 66

10.3 Symotry propertios of the total wave function 67

10.4 The cnergy leved diagran of the asymetric top rolecule in the $\mathrm{C}_{2 \mathrm{~h}}$ point groun $\quad 71$ 
Chapter 11: Rotation-Vibration Solection Rules for Asymotric Top Molecules

11.1 Infrared rotation-vibration selection

rules for asymatric top nolecules

11.2 Theoretical infrared band envelopes of asymetric rotor molecules

11.3 Naman rotation-vibration selection rules for the esymatric top nolecules

Chapter 12: Vibretional wodes of Oxalic Actd and Review of Previous Hork

12.1 Vibrational modes of gaseous oxalic acid

12.2 Reviow of previous work on gaseous oralic acid 83

12.3 Sumbary of previous work on solid oxalic acid and solutions

Chapter 13: Physical and Chenical Propertios of Oxalic Acid

13.1 Dhysical and ciemical properties 93

13.2 Vapour pressure of anhydrous oxalic acld 95

13.3 Molocular structure of gaseous oxalic acta 98

Chapter 14: Principa1 Woments of Inertia and Principal Axes of Gaseous Oxalic Acid

14.1 (a) Principal moments of incrtia of the gascous oxalic acid first model

(b) Principal axes of the gaseous oxalic acid first modol

14.2 Principal moments of inertia and the principal axos of gaseous oxalic acid second model $\quad 106$

Chapter 15: Tho G-Matrix of the Inplano Vibrations of Gaseous Oxalic Acid Fixst lodel

15.1 Evaluation of the is matrix of the inplane vibrations

15.2 D matrix eloments of the inplane vibration of gaseous oxalic acid first model 
15.3 6 matrix eloments in intemal co-ordinates of the inplane vibration of gaseous oxalic acid first model

15.4 Symotry co-ordinatcs of the inplanc vibration of gaseous oxalic ecid first model

15.5 Numorical values of the $G$ ratrix first nodel in symetry co-ordinates

Chapter 10: Construction of the Filatrix of the Inplane Vibrations of Gaseous Oxalic Acid First Mode1

16.1 Urey-iradley forco field of gaseous oxalic acid first model

16.2 Evaluation of F pactix

16.3 F netrix in intcrnal co-ordinetes of gaseous oralic acid first modol

16.4 F matrix in symetry co-ordinates of gaseous oxalic acid first model

Chapter 17: G-Matrix of the Inplane Vibrations of Gascous Oxalic Acid Sccond Model

17.1 Evaluation of the $\mathrm{B}$ matrix of the inplane vibrations of gaseous oxalic acid second mode 1

17.2. D antrix of the inplane vibrations of gascous oxalic acid second nodel

17.3 $G$ matrix in intomal comordinates of the inplane vibrations of gaseous oxalic acid second nodel

17.4 Symotry co-ordinates of the inplane vibrations of gaseous oxalic acid second mode1

17.5 Numerical values of 6 matrix second nodel in symetry co-ordinates

Chapter 18: Construction of the Folatrix of the Inplane Vibrations of Gaseous Oxalic Acid Second ibdel

18.1 Urcy-Bradicy force field of gastous oxalic acia sccond nodel. 
18.2 Evaluation of the F natrix

18.3 $\mathrm{F}$ natrix in intenal co-ordinates of the gaseous oxalic acia second model

18.4 F matrix in symetry co-ordinates of geseous oxalic acid second model

Chapter 19: The Infrared Experiment

10.1 The infrared instrument

19.2 Developing the heated coll and the additional optical systent

19.3 Infrared experiment 204

19.4 Infrared results and the assignment 207

19.5 The expected and observed band envelopes of gescous oxalic acid

Chapter 20: Ranan Experinont

20.1 Laser Raman instmmant 243

20.2 Raman oven 246

20.3 Ranan experinent 252

20.4 Rasan rosults and the assigntent 256

20.5 Comperison between infrared and lantan
exporiments 279

Chapter 21: Far-Infrared Exporiment $\quad 280$

Chapter 22: Calculation and the Assignment of the Observed Bands of Goscous Oxalic Acid

22.1 The implane force constants and the calculated frequencies of gasoous oxalic acid 282

22.2 Calculation using first nodel 283

22.3 Assignnent of the observed infrered and Raman bands, potential onergy distribution and nomel tiodes 288

22.4 Calculation using socond nodel 304 
22.5 Assigunent of the observed infrarcd ma Raman bands, potential energy distribution and nomel moles

22.6 The expected frequencies of deuterated oxalic acid nononer

22.7 The out of plane frequencies 328

Chapter 23: Conclusions and suggestions for future work 329 
INTRODUCTION

Oxalic acid exists in throe solid forns, $\alpha, \beta$, and the dilydrate. The structures have been studied using $X$-rays and Neutron diffraction methods.

Many vibrational studies of carboxylic acids have boon carxied out and complete assignent of the vibrational nodes of formic and acetic acids have been made. Several papers have been published on the infrared and Raman spectra of oxalic acid in both solution and tho solid phase. Hone of then give complete spectra and their assignant. This my be due to the following reasons:-

(1) There is no complete work on the infrared and Ranan spectra of the free nolccule.

(2) In the condensed pliases the vibrational spectmm is not only affected by intramolecular forces but also by internolecular forces.

(3) In solution the spectrum is influnced by the solvent effect.

(4) All forms of solid oxalic acid are hydrogen bonded into polymers. Thus, their spectra are strongly influenced by the hydrogen boncis.

It is clear fron the above that the vibrational modes of oxalic acid in condensed phases aro quite complicated. Differences in their structuros result in the differences in their vibrational spectra. Tixis was clearly shom in the spectra of Bollamy and Pace in $1963(62)$. 
In order to be able to complete the work of assignment and interpretation a knowledge of the vibrational nodes of the free molecules is roquired. With this in mind infrared and Rarazn spectra of gaseous oxalic acid were studied and the results are presented in this thesis.

Before this work started a paper on the infrared spoctrum of gaseous oxalic acid was publishod by pava and Stafford (98). Three bands were observed and were assigned to gasoous oxalic acid. The second peper appoared turing the courso of this wort. This papor was publishod by Bardet, Fleury and Tabacik (109) on the infrared absorption bands of gaseous oxalic acid and the Ranan bands of oxalic acid in solution and in the solid phase. They recorded more infrared gaseous oxalic acid bands. Unfortunately this paper was badly writton and essignments of somo gaseous oxalic actd bands aro wrong and sone are very dowtful as pointed out in section 12.2 .

No Ranan spectrum of gaseous oxalic acid has previously been reportod.

Two papars were published on the structure of gaseous oxalic acia using the electron diffraction mathod. The first paper was published by Shibata and Mimura (104) beforo this work startod. The second paper appeared during the course of this work. This latter paper was published by Nahlovska, Nahlovsky and Strand (78). Both papers interproted their results on the besis of a planar trans conformation. Nio possible rodels due to the positions of II atons were suggested by the lacter paper (soe section 13.3). 
I

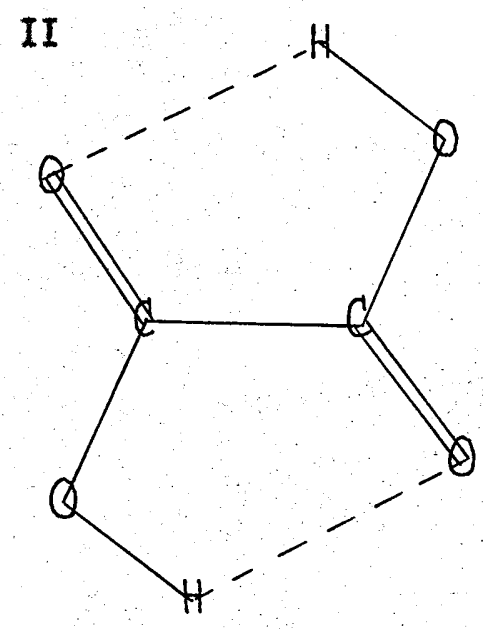

The force field calculations were made using both models for the reason that some evidence may be obtained to distinguish between them. In fact this problen had not been expected before this work started.

An initial set of force constants for gaseous oxalic acid was taken fron the values for gaseous acetic acid published by Nakamoto and Kishida (39).

A Urey-Bradley force field was used for the implane frequancies.

Vibrational frequencies, potential energy distributions and nornal modes were computed for both structures and also for the deuterated forms. 


\section{CLAPTER 1}

\section{MOTION IN CARTESIAN CO-ORDINATES}

1.1 Quantum mechanical description on the basis of the hamonic oscillator model

According to the Bom-Oppenheimer approximation the total wave function for a molecule may be taken as an approximation to be

$$
\Psi=\psi_{\mathrm{E}} \psi_{\mathrm{V}} \psi_{\mathrm{R}} \psi_{\mathrm{T}}
$$

where $\psi_{E}, \psi_{V}, \psi_{\mathrm{R}}, \psi_{\mathrm{T}}$ are the electronic, vibrational, rotational and translational wave functions respectively. Here we are concemed only with the vibrational wave function $\psi_{v^{*}}$ The wave equation in nomal co-ordinetes will then have the form

$$
-\frac{h^{2}}{8 \pi^{2}} \sum_{k=1}^{3 N-6} \frac{\delta^{2} \psi_{v}}{\delta Q_{k}^{2}}+2 \sum_{k=1}^{3 N-6} \lambda_{k} Q_{k}^{2} \psi_{v}=v_{v} \psi_{v} \ldots \ldots 1.2
$$

where $W_{v}$ is the vibrational energy. The wave equation in this form is separalle into $3 \mathrm{~N}-6$ equations, one for each nornal co-ordinate. So

$$
w_{v}=W(1)+W(2)+\ldots+W(3 N-6)
$$


and

$$
\psi_{v}=\psi\left(Q_{1}\right) \cdot \psi\left(Q_{2}\right) \ldots \ldots \psi\left(Q_{3 N-5}\right)
$$

Then it will be seen that the wave equation is satisfied if the function $\psi\left(Q_{k}\right)$ and the energy $w_{k}$ satisfy equations of the type

$$
-\frac{n^{2}}{8 \pi^{2}} \frac{d^{2} \psi\left(Q_{k}\right)}{d Q_{k}^{2}}+\frac{1}{2} \lambda_{k} Q_{k}^{2} \psi\left(Q_{k}\right)=w_{k} \psi\left(Q_{k}\right) \quad \ldots \ldots 1.5
$$

Each of these equations is a differential equation in one variable, $\mathrm{Q}_{\mathrm{k}}$. In fact this equation is the vell-known wave equation for the linear hamonic oscillator, expressed in terms of the nornal co-ordinate $Q_{k}$. The solution $\psi_{v}$ of the vibrational problen is therefore expressible, as a product of hamonic oscillator functions $\psi\left(Q_{k}\right)$ while the total vibrational energ $W_{v}$ is the sum of the energies of $3 N^{-6} 6$ harronic oscillators.

The solutions of the harmonic oscillator equation are calied Hernite orthogonal functions and are of the form

$$
\psi_{v_{k}}\left(Q_{k}\right)=N_{v_{k}} e^{-\frac{1}{2} r_{k} Q^{2} k} H_{v_{k}}\left(r_{k}^{\frac{1}{2}} Q_{k}\right) \quad \ldots \ldots 1.6
$$

in which $N_{v_{k}}$ is the nomalising factor

$$
N_{v_{k}}=\left[\left(\frac{r_{k}}{\pi}\right)^{\frac{1}{2}} \frac{1}{2^{v_{k}\left(v_{k} !\right)}}\right]^{\frac{1}{2}}
$$


$r_{k}$ is the quantity $4 \pi^{2} v_{k} / h$ and $h_{v_{k}}\left(r^{\frac{1}{3}}{ }_{k} Q_{k}\right)$ is the Hernite polynomial of degroe $v_{k}$ in $Q_{k}$.

The energy levels of a linear harmonic oscillator are given by the expression

$$
W_{v}=(v+1) h v
$$

where $v$ is the vibrational quantum number. Consequently, the vibrational energy of a molecule with several harmonic frequencies $v_{k}$ is of the form

$$
\begin{aligned}
W= & \left(v_{1}+\frac{1}{2}\right) h v_{1}+\left(v_{2}+\frac{1}{2}\right) h v_{2}+\ldots \cdot \cdots \\
& +\left(v_{3 N-6}+\frac{1}{2}\right) h v(3 N-6)
\end{aligned}
$$

That is, every nomal co-ordinate $Q_{k}$ has associated with it a quantun number $v_{k}$ and a nomal frequency $v_{k}$ *

\subsection{Normal modes of vibration}

If we assume that the particles behave as harmonic oscillators, each atom is oscillating about its equilibriun position with a simple hamonic motion according to the oquation

$$
q_{i}=A_{i} \cos \left(\lambda^{\frac{1}{2}} t+c\right)
$$

The frequency $\lambda^{\frac{1}{2}} / 2 \pi$ and the phase of motion of each atoms is the same, but the asplitudes $A_{i}$ may be, and usually are, 
different for each co-ordinate $q_{i}$. On account of the equality in phase and froquency, each atom reaches its position of maximum displacement at the same tims and each atom passes through its equilibrium position at the same time. A mode of vibration having all theso characteristics is called a nomal mode of vibration, and its frequency is knom as a normal, or fumdanontal, frequency of the molecule.

The number of degrees of freedon possessed by the molecule is the number of the co-orainates required to specify completely the position of the nuclei. Each nucleus requires 3 co-ordinates to define its position and so a molecule of $N$ atons has $3 N$ degrees of freedora. Three are accounted for by the translational motion of the centre of mass and mother three are accounted for by the rotational motion about the centre of mass. So the romaining $\left(3 y^{-6)}\right.$ are associated.with the internal vibrational motion of the atoms in the non-linear nolecule. Each vibrational degree of freedon corresponds to a normal mode of vibration of the molecule.

\subsection{Motion in cartesian displacernent co-ordinates}

There is no general theory or technique for dealing with an anhamonic force field in molecules, and thus, in order to be able to solve the vibrational problen (i.c. calculating vibrational frequeacies) it is necessary to assume that the vibration of trolecules is harmonic (5).

Consider $N$ nuclei at their equilibrium positions with cartesian axes attached. The displacenents of all nuclei are 
defined by $3 N$ displacenent co-ordinates, $\Delta x_{1}, \Delta y_{1}, \Delta z_{1}, \Delta x_{2}, \Delta y_{2}$, $\Delta z_{2}, \ldots . \Delta x_{N^{\prime}} \Delta y_{i N}, \Delta z_{N^{*}}$ For simplicity we used $q_{i}^{\prime}$ as cartesian displacenent co-ordinates so that $q_{i}^{\prime}$ runs from 1 to 3N.

The kinctic energy of the molecule in cartesian displacement co-ordinates is

$$
2 T=\varepsilon_{i} m_{i} \dot{q}_{i}^{2}
$$

The potential field in which the nuclei nove is a function of the displacentent co-ordinates.

$$
v=v\left(q_{1}^{\prime}, q_{2}^{\prime}, q_{3}^{\prime} \ldots . . q_{3 N}^{\prime}\right)
$$

The potential function $V$ may alvays be expanded in a Taylor series In the $q_{i}^{\prime}$ about the equilibriun positions of the nuclel. Using the subscript zero to denote the equilibrium position gives

$$
V=V_{0}+\sum_{i=1}^{3 N}\left(\frac{\delta V}{\delta q_{i}^{\prime}}\right)_{0} q_{i}^{\prime}+1 \sum_{i, j=1}^{3 N}\left(\frac{\delta^{2} V}{\delta q_{i}^{\prime} \delta q_{j}^{\top}}\right)_{0} q_{i}^{\prime} q_{j}^{\prime}+\ldots
$$

If higher tems in this expression are ignored for small displacements this corresponds to an harmonic potential function. The potential energy is masured relative to the value when the atoms are in their equilibrium positions, hence $V_{0}=0$. The equilibrium positions correspond to a stable minima in the potential 
energy surface, hence all first derivatives are zero, that is,

$$
\left(\frac{\partial V}{\delta q_{i}}\right)_{0}=0
$$

The resulting potential function is

$$
2 V=\sum_{i, j=1}^{3 v}\left(\frac{\delta^{2} V}{\delta q_{i}^{6 q} q_{j}}\right)_{0} q_{i}^{\prime} q_{j}^{i}
$$

By Taylor's Theoren

$$
\begin{aligned}
& \left(\frac{\delta^{2} v}{\delta q_{i}^{\prime} q_{j}^{\prime}}\right)_{0}=\text { constant } \\
& \therefore 2 v=\sum_{i, j=1}^{3 N} b_{i j}^{\prime} q_{i}^{\prime} q_{j}^{\prime}
\end{aligned}
$$

where $b^{\prime}{ }_{i j}=b^{\prime}{ }_{j i}$

1.4 Motion in rasss weighted cartesian displacenent co-ordinates

$$
\text { If we define } q_{i}=\sqrt{m_{i}} q_{i}^{\prime}
$$

The kinetic energy exprossion becones $2 T=\Sigma \dot{q}_{i}{ }^{2}$

The potential energy expression becones

$$
2 V=\sum_{i, j=1}^{3 N} b_{i j} a_{i} q_{i j}
$$

where $b_{i j}=b^{\prime}{ }_{i j} /{ }_{m_{i} m_{j}}$ and $b_{i j}=b_{j i}$

Thelagrangian equation of notion is

$$
\frac{d}{d t}\left(\frac{\delta L}{\delta \dot{q}_{i}}\right)-\frac{\delta L}{\delta q_{i}}=0
$$<smiles>C[13CH][18OH]</smiles>

where $L=T-V$. 
On substituting 1.17 and 1.18 in 1.19 , the equation gives a set of $3 \mathrm{k}$ linear second order homogeneous differential equations

$$
\ddot{q}_{i}+\sum_{j}^{3 N} b_{i j} a_{j}=0
$$

The solution of this form of differential equation may be writton as

$$
q_{i}=A_{i} \cos \left(\lambda^{\frac{3}{2}} \tau+\varepsilon\right)
$$

where $A_{i}$ is the arplitudo, $\lambda^{\frac{1}{2}} / 2 \pi$ is the Erequency, and $\varepsilon$ is the phase angle.

The 3 is values of $\lambda$ may be evaluated by differentiation and substituting back into 1.20. This gives

$$
\sum_{j}^{3 N} b_{i j} A_{j}-\lambda A_{i}=0
$$

which can bo roarranged into

$$
\sum_{j \neq i}^{3 N} b_{i j} A_{j}+\left(b_{i j}-\lambda\right) A_{i}=0
$$

Homogeneous simultaneous equations have solutions other then the trivial one $A_{1}=A_{2}=A_{3}=\ldots .0$ if the determinent of the coefficients is zoro. Hence 


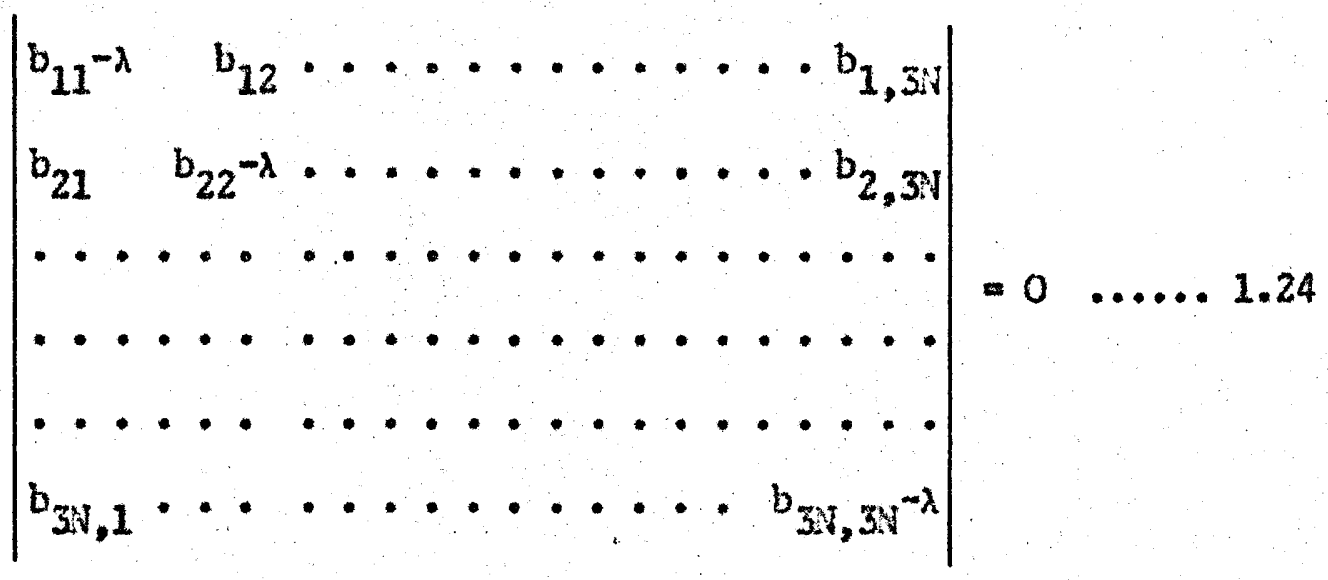

which nay be vititen as

$$
\left|b_{i j}-\lambda \delta_{i j}\right|=0
$$

Equation 1.24 has $3 N$ roots winch are the values of $\lambda$ which cause the determinant to vanish. For a non-linear molecule six roots will be zero and for a linear nolecule 5 roots will be zero. These zero roots represent translational and rotational motions of the molecule which have zero frequency of vibration.

\subsection{Detomination of the form of the nomal modes}

Let one of the roots be $\lambda_{k}$. Substitution in 1.24 gives $3 \mathrm{~N}$ linear homogeneous equations and therefore can only be solved to give the ratio between the amplitudes $A_{i k}$. These amplitudes do not define unique values, which is consistent with the fact that we are dealing with hnonic motion and the frequency is independent of the amplitude.

For convenience a set of nomalised anplitudes are used. Theso can be calculated such that they are in tire same ratio as 
the $A_{1 k^{*}}$ If the constant of proportionality is $\mathrm{K}_{\mathrm{k}}$ then

$$
A_{i k}=K_{k i k}
$$

and the norwalisation condition is that

$$
2 \ell^{2} 1 k=1
$$

So that there is a colum vector $l_{i k}$ corrosponding to the $\lambda_{k}$ * The colum vectors for all $\lambda$ can be written to form a matrix $L$ of dimonsion $3 \mathrm{~N}$ in which

$$
x=10
$$

where $X$ = matrix representing cartesian displacenent co-orcinates

$$
Q=\text { natrix representing nomal co-ordinates }
$$

and

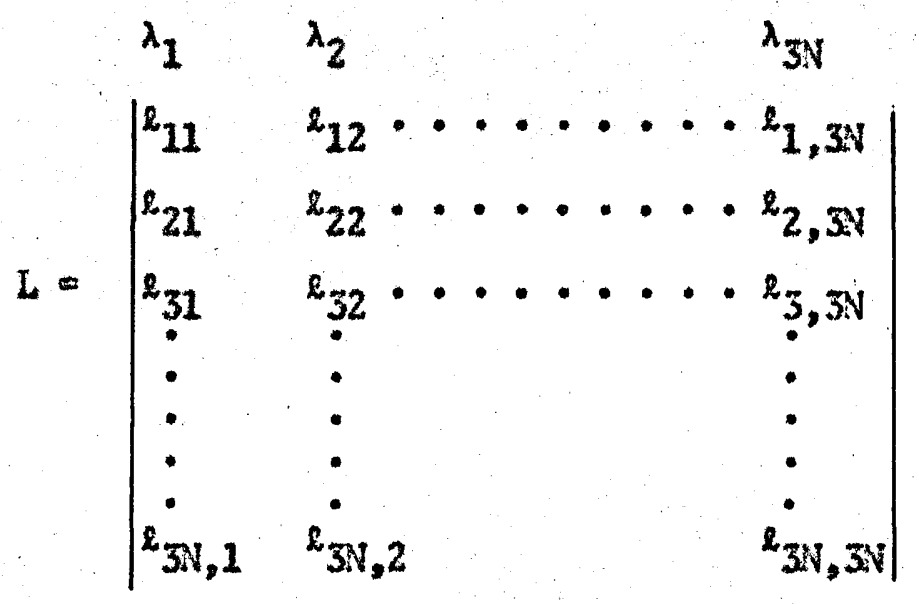


One can construct vectors, attached to each atom, to show the relative magnitude and direction of the displacenonts from the values $\ell_{i k}$. That is from $2_{1 k},{ }^{2} 2 k, \ell_{3 k}$ for atom $1, \ell_{4 k}, \ell_{5 k}$, $\ell_{6 k}$ for atom $2, \ldots . .$, and $\ell_{3 N-2, k}, \ell_{3 N-1, k}, \ell_{3 N, k}$ for aton $N$ for the kth vibration corresponding to $\lambda_{k^{*}}$ Such diagrans ropresent nomal modes of vibration. 


\section{GIATTER 2}

BDTIOU IN INTERNAL OD-ORDINATES

\subsection{Kinetic energy in terms of internal co-ordinates}

The cartesian displacenent co-ordinates can be transformed

into internal co-ordinates $k$ by the equation

$$
\begin{aligned}
\text { or } \quad \mathrm{R} & =\mathrm{Bq} \\
& =\mathrm{DQ}
\end{aligned}
$$

(.....2.1

(.....2.2 2.2

where $\quad R$ a natrix representing internal co-ordinates

$q^{\prime}=$ matrix representing cartesian displacoment co-ordinates

$q=$ matrix representing mass-weighted cartesian displacement co-ordinates

$B$ and $D$ are matrices of transformations from cartesian co-ordinates to internal co-ordinates in which

$D=B m^{-\frac{1}{2}}$

in a diagonal matrix of nasses

In terms of mass-reighted cartesian displacement co-ordinates, the kinetic energy is given by

$$
2 T=q^{t} q^{*}
$$

If $p_{j}$ is the momentum conjugate to $q_{i}$

$$
p_{j}=\frac{\delta T}{\delta q_{j}}=q_{j}
$$

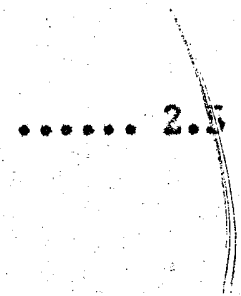


Thus, the kinetic energy can be simply written in terms of the monenta as

$$
2 T=p_{p}
$$

$\ldots \ldots 2.6$

Suppose that $\mathrm{T}$ is now considered as a function of the velocities in the internal co-ordinates. Using the rules for partial differontiation (1)

$$
p_{j}=\frac{\delta T}{\delta q_{j}}=\Sigma_{n} \frac{\delta T}{\delta k_{n}} \cdot \frac{\delta \dot{R}_{n}}{\delta \dot{q}_{j}}
$$

Since $\frac{\delta T}{\delta P_{n}}=P_{n}$ and $\frac{\delta p_{n}}{\delta q_{j}}=p_{n j}$

then equation 2.7 becomes in ratrix fora

$$
p=D^{t} p
$$

a....2.8

and

$$
p^{t}=p^{t}
$$

..... 2.9

Substituting 2.8 and 2.9 in 2.6 , the equation becones

$$
\begin{aligned}
2 T & =p^{t}(D D) P \\
& =p^{t} C P
\end{aligned}
$$


If $|G| \neq 0, G^{-1}$ exists and by Hamilton's equations

$$
\dot{\mathrm{R}}_{\mathrm{n}}=\frac{\delta \mathrm{T}}{\delta \mathrm{P}_{\mathrm{n}}}
$$

then

$$
\dot{R}=\mathrm{QP}
$$

which can be solved for $P$

$$
P=G^{-I} \dot{R}
$$

Thus, the kinetic energy in tems of the intemal co-ordinates is

$$
2 T=\dot{R}^{t} G^{-1} \dot{R}
$$

2.2 Potential energy in tems of internal co-ordinates

The potential energy of a vibrating molecule can be expanded as a power series in the intenal co-ordinate system by Taylor's theorem

$$
V=V_{0}+\sum_{i=1}^{n}\left(\frac{\delta V}{\delta R_{i}}\right)_{0} R_{i}+\frac{1}{2} \sum_{i, j}^{n}\left(\frac{\delta^{2} V}{\delta R_{i} \delta R_{j}}\right)_{0} R_{i} R_{j}+\text { higher terms }
$$

where $\mathrm{n}$ is the number of internal co-ordinates and the subscript o donotes tho equilibrium position. 
Assuning an hamonic potential function the higher toms in 2.10 can be ignored. $V_{0}=0$ when the potential energy is ineasured relative to the value then the atoms are in their cquilibrium position. At equilibrium the potential energy must be minimun, nence

$$
\left(\frac{\partial V}{\delta R_{i}}\right)_{0}=0 \text { for } i=1,2, \ldots \ldots n
$$

The resulting potontial function for the hamonic oscillator nodeI is

$$
V= \pm \sum_{i, j=1}^{n}\left(\frac{\delta^{2} V}{\delta R_{i} \delta R_{j}}\right)_{0} R_{i} R_{j}
$$

By Taylor's theoren

$$
\left(\frac{\delta^{2} V}{\delta R_{i} R_{j}}\right)_{0}=E_{i j}=f_{j i}
$$

where the $f^{\prime} s$ are the force constants in intemal comordinates. The potential energy can be cxprossed as

$$
2 V=\sum_{i, j=1}^{n} \hat{x}_{i j} R_{i} n_{j}
$$

In matrix form equation 2.19 beconss

$$
2 V=R^{t} \geqslant R
$$

where $F$ is the matrix representing the force constants $f_{i j}$. 
2.3 The secular equation in termis of intemal co-ordinates

Both the kinetic enerty and the potential energy can be

expressed in the same interal co-orainates as

$$
\begin{aligned}
& 2 T=R^{t} G^{-1} R \\
& 2 V=R^{t} F R
\end{aligned}
$$

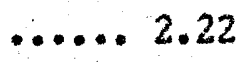

From Lagrange's equation of notion

$$
\frac{d}{d t}\left(\delta \mathrm{T} / \delta \dot{R}_{i}\right)+\delta V / \delta R_{i}=0
$$

Substituting 2.21 and 2.22 in 2.23 the cquation becomes

$$
\sum_{j=1}^{n}\left(\left(G^{-1}\right) \ddot{R}_{j}+f_{i j} R_{j}\right)=0
$$

whero $n$ is the number of internal co-ordinates.

Equation 2.24 forms in honogeneous second order differential equations for which the solutions are

$$
R_{i}=A_{i} \cos \left(\lambda^{\frac{1}{2} t}+\varepsilon\right)
$$

where $\lambda_{i}$ is the atginitude, $\lambda^{\frac{1}{3}} / 2 \pi$ is the frequency, and $\varepsilon$ is phase anglo. 
Substituting 2.25 into 2.24 the vibrational problen leads to a secular equation

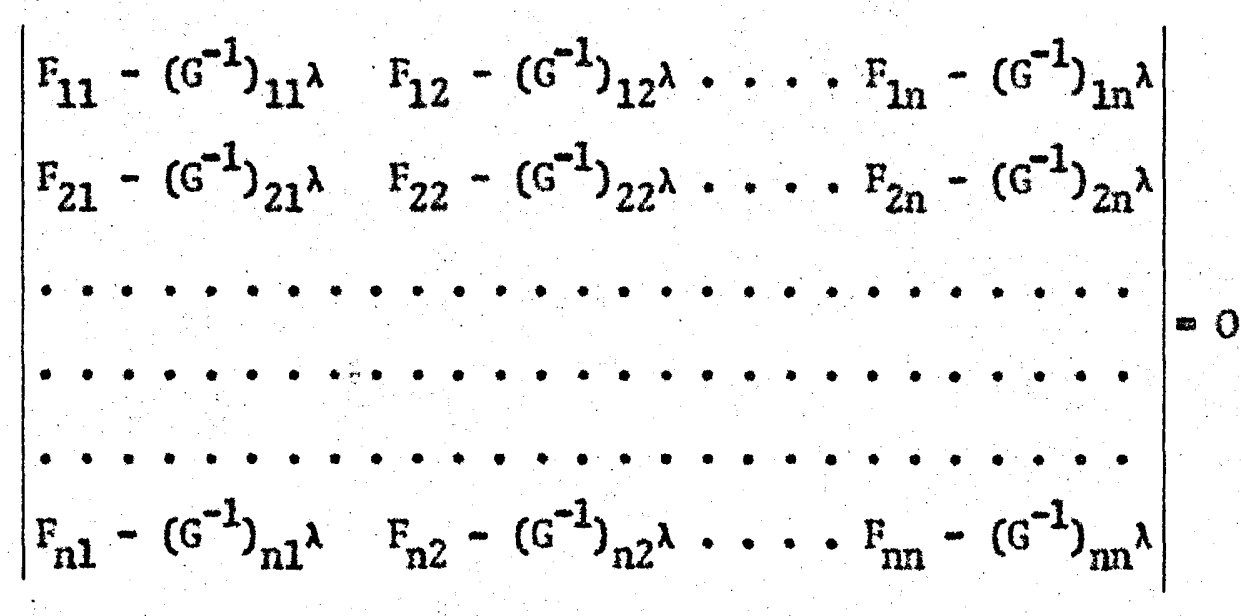

Here $\lambda=4 \pi^{2} v^{2}$.

Symbolically equation 2.26 can be written as

$$
\left|F-G^{-1} \lambda\right|=0
$$

Another form of the secular equation can be obtained by multiplying 2.27 by the deteminant of $G$, that is, by $|G|$, using the rules for the multiplication of deterninants. The secular equation becones

$$
|G F-E \lambda|=0
$$

where $\mathrm{E}$ is the unit natrix ( 3 page $63-65$ ).

Because both $F$ and $G$ are symatrical, rows and colums in 2.28 can be interchanged to yield another form which can be written as

$$
|F G-E \lambda|=0
$$




\section{CHAPTER 3}

MOTION IN SYMETRY CO-ORDINATES

\subsection{Symoetry co-ordinates}

Symetry co-ordinates may be described as follows:-

(1) They are related to the intemal co-ordinates by the transformation

$$
S=U R
$$

where $S$ matrix representing symatry co-ordinates

$R=$ matrix representing intemal co-ordinates

$U=$ matrix of transfomation.

In othor words, they are linear cosbinations of internal co-ordinates.

(2) They must thenselves form representations for the class of vibrations for which they are to be used. This means that the co-ordinates must have the same symetry and antisymetry properties as the vibrational modes themselves (1).

(3) They mast form a complete orthogonal set that is

$$
\sum_{k} s_{j k} s_{k k}=0
$$

$\ell$ and $j$ refer to different symetry co-ordinates (4).

(4) The number of symatry co-ordinates will equal the nunber of fundanental vibrations. If it exceeds the number of vibrations then this implies a redudancy, that is, certain 
combinations of these internal co-ordinates will be equivalent to no vibrational inotion at all and therofore correspond to a zero frequency (1).

(5) With these co-ordinates the vibrational problem for each syninetry class can be solved independently (3). Under symatry co-ordinates both kinetic and potential crergies have the form

$$
\begin{aligned}
& 2 T=\dot{S}^{t} G^{-1} \dot{S} \\
& 2 V=S^{t} \underline{S}
\end{aligned}
$$$$
\text { (.... } 3.4
$$

Substituting 3.1 into 3.3 and 3.4 wo have

$$
\begin{aligned}
& 2 T=\dot{R} U^{t} \underline{G}^{-1} \dot{U R} \\
& 2 V=R U^{t} \underline{F U R}
\end{aligned}
$$

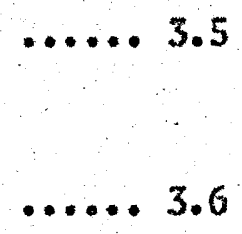

On comparing 3.5 and 3.6 with 2.21 and 2.22 we have

$$
\begin{aligned}
& G=\text { UGU }^{t} \\
& \underline{F}=\mathrm{UFU}^{t}
\end{aligned}
$$


If $s_{j}$ and $s_{k}$ belong to different symetry types there will be at least one symetry operation with respect to which $S_{j}$ and $s_{k}$ behave differently, that is, there will be at least one symetry operation for which $S_{j} S_{k}$ changes sign. Since the potential energy nust be invariant with respect to all symetry operations, it follows that $g_{j . k}$ and $f_{j . k}$ are zero whenever $s_{j}$ and $S_{k}$ belong to different species (2). Therefore the secular equation

$$
|\underline{G} \underline{E}-\mathrm{EX}|=0
$$

assumes the block form as indicated in Fig. 1 where all elements outside the shaded area are zero, since they correspond to $s_{j}$ and $S_{k}$ of different species. Each shaded square corresponds to one species. The determinant can now be written as a product of factors corresponding to each species. Fach of those factors put equal to zero gives the normal frequencies of these species.

\section{Fig. 1}

Form of secular determinant set up

in tenns of symetry co-ordinates

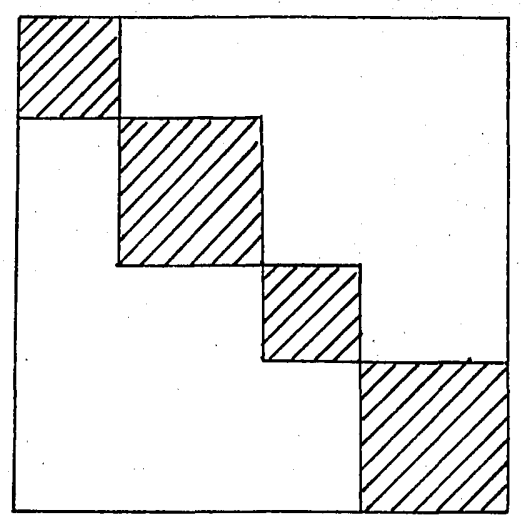




\subsection{Construction of symatry co-ordinates}

Symetry co-ordinates may bo written as

$$
s_{j}=\sum_{k} u_{j k} x_{k}
$$

where $S_{j}$ is the $j$ th symnetry co-ordinate, $x_{k}$ is the kth internal co-ordinate, and $U_{j k}$ is the suitable coefficient for $r_{\mathfrak{k}^{*}}$. In natrix notation equation 3.10 becomes

$$
S=U R
$$

The symetry co-ordinates selected are not necessarily unique but they must have definite properties. Since they are not unique, a perfectly general method for obtaining then cannot be given (4). A simple rule for construction of trial symatry co-ordinates was described by Wilson, Decius and Cross ( 3 pp 117 121) using the expression

$$
s^{A}=N \sum_{\hat{R}} x_{\hat{R}} \hat{R} s_{i}
$$

where $S^{A}$ is the symetry co-ordinate of the vibration species $A$ (Ag, etc.), $x_{\hat{R}}$ is the character corresponding to $\hat{R}$ of the vibration of species $A, \hat{R}$ is the operator in the point group, $s_{i}$ is the generating co-ordinate, and $N$ is the normalising factor. The generating co-ordinate $S_{i}$ may be a single internal co-ordinate or linear combination of intemal co-ordinates. 
The trial symetry co-ordinates must be nomal, orthogonal, and they nust transform properly (4). The condition for nomality is

$$
\sum_{k} u_{j k} u_{j k}=1
$$

where $U_{j k}$ is a coefficient in the $j$ th symuetry co-ordinate and the sumation is carried over the $k$ intemal co-ordinates. The condition therefore requires the sum of the squares of the coefficients in each symetry co-ordinate to be one.

The condition for orthogonality is

$$
\sum_{k} u_{j k} u_{2 k}=0
$$

where $\ell$ and $j$ refer to different symetry co-ordinates. That is, the sur of the products of the coefficients in the corresponding internal co-ordinates (same intemal co-ordinates) of two difforent symetry co-ordinates is equal to zero and this must be true for every pair of symuetry co-ordinates. 


\section{CHAPTER 4}

BOTION IN HORAAL CO-ORDINATES

The normal co-ordinates $q_{k}$ can be cxpressed in terms of the nass-weighted cartesian displacenent co-ordinates $q_{i}$ by the linear equation

$$
Q_{k} \cdot \sum_{i=1}^{3 N} e_{k i} q_{i}
$$

in which the coefficients $\&_{k i}$ have been chosen so that in terms of the new co-ordinates the kinetic and potential energies have the forms

$$
\begin{aligned}
& 2 \mathrm{~T}=\sum_{\mathrm{k}} \dot{\mathrm{Q}}_{\mathrm{k}} \\
& 2 \mathrm{~V}=\sum_{\mathrm{k}} \lambda Q^{2} \mathrm{k}
\end{aligned}
$$

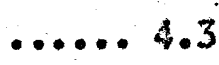

In other worls, the potential energy in tems of the nornal co-ordinates involves no cross products but only squares of Q's.

In matrix notation equations 4.2 and 4.3 become

$$
\begin{aligned}
& 2 T=\dot{Q}^{t} \dot{Q} \\
& 2 V=Q^{t} \wedge Q
\end{aligned}
$$

where $A$ is the diagonal matrix of the $\lambda$ values.

The transformation between the mass-weighted cartesian co-ordinates and the normal co-ordinates is an orthogonal trans- 
formation. This can be proved as follows:-

$$
\begin{aligned}
& 2 T=\dot{Q}^{t} \dot{Q} \\
& \text { - } e^{t} e^{t} d \dot{a} \\
& =a^{t} l_{1} \text { only if } \ell^{t}=e^{-1}
\end{aligned}
$$

That is, 2 is an orthogonal natrix.

The diagrans which represent normal nodes of motion can also be used to represent normal co-ordinates if the vectors are dratw so as to represent displacenents, not only in ordinary wits, but in the mass-adjusted scale of co-ordinates $q_{i}$. Then the component of a vector along the direction of co-ordinates $q_{i}$ is proportional to $\ell_{i k^{*}}$. That is, the diagran represents not only the relative amplitudes of the atoms during the nomal mode of notion, but also the coefficients in the transformation.

4.2 Detemination of nomal co-ordinates

For a vibrating molecule there will be only one nomal co-ordinate $Q_{k}$ essociated with one mode of vibration $v_{k}$. The nomal co-ordinate is related to the internal co-ordinates by

$$
R=L Q
$$

$$
\text { where } \begin{aligned}
R & =\text { matrix of internal co-ordinates } \\
L & =\text { matrix of transfomation } \\
Q & =\text { matrix of nomal co-ordinates }
\end{aligned}
$$


$L$ is chosen so that

$$
\begin{aligned}
& 2 T=\dot{Q}^{t} Q \\
& 2 V=Q^{t} A Q
\end{aligned}
$$

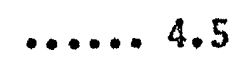

(....4.6

whore $\Lambda$ is the diagonal matrix of the latent roots. Substituting 4.4 in 4.5 and 4.6 we have

$$
\begin{aligned}
& 2 T=\dot{R}^{t}\left(L^{-1}\right)^{t} L^{-1} \dot{R} \\
& 2 V=R^{t}\left(L^{-1}\right)^{t} A L^{-1} R
\end{aligned}
$$

......4.8

Comparing 4.7 and 4.8 with 2.21 and 2.22 we have

$$
G=L L^{t}
$$

$$
\text { and } \Lambda=L^{t_{F L}}
$$

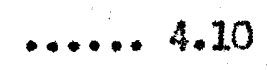

Application of Newton's equations of motion in the Lagrange forn and substitution of a trial periodic solution of the form

$$
R_{i}=A_{i} \cos \left(\lambda^{\frac{1}{2}} t+c\right)
$$

where $A_{i}$ is the amplitude, $\lambda^{\frac{1}{3}} / 2 \pi$ is the froquency, and $\varepsilon$ is phase angle, leads to the equations 


$$
\begin{aligned}
& {\left[\mathrm{F}_{11}-\left(G^{-1}\right)_{11^{\lambda}}\right] A_{1}+\left[\mathrm{F}_{12}-\left(G^{-1}\right)_{12}\right] A_{2} \ldots \cdot\left[F_{1 n}-\left(G^{-1}\right)_{1 n^{\lambda}}\right] A_{n}=0} \\
& {\left[E_{21}-\left(G^{-1}\right)_{21}\right] A_{1}+\left[F_{22}-\left(G^{-1}\right)_{22^{\lambda}}\right] A_{2}+\cdots\left[F_{2 n}-\left(G^{-1}\right)_{2 n} \lambda\right] A_{n}=0}
\end{aligned}
$$

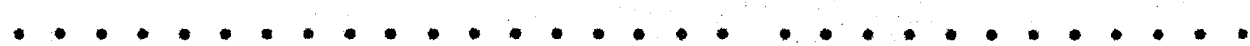

$$
\begin{aligned}
& \ldots \ldots \ldots \ldots \ldots \ldots \ldots \\
& \left.\left[F_{n 1}-\left(G^{-1}\right)_{n 1^{\lambda}}\right] A_{1}+\left[F_{n 2}-\left(G^{-1}\right)_{n 2}\right] A_{2} \cdots\left[F_{n n^{-(G}} G^{-1}\right)_{n}\right] A_{n}=0
\end{aligned}
$$

For a given value of $\lambda$, these equations can be solved for the relative anplitudes of $A_{i}$.

In natrix notation 4.12 becomes

$$
\left(F-G^{-1} \lambda_{k}\right) A_{k}=0
$$

Equation 4.13 can be transformed into

$$
\left(G F-E \lambda_{k}\right) L_{k}=0
$$

where $k_{i k}-N_{k} A_{i k}$ and $L_{k}$ is the $k$ th columa of the matrix $L_{i k}$.

Solution of these equations for the normalised $\mathrm{L}_{\mathrm{ik}}$ can be carried out (3pp $71-74)$.

\subsection{Potential energy distribution}

The potential energy of a molecule in symetry co-ordinates can be written as

$$
2 \mathrm{~V}=\mathrm{S}^{\mathrm{t}} \mathrm{F} \cdot \mathrm{S}
$$


in which symatry co-ordinates and nomal co-ordinates are related by the equation

$$
S=L
$$

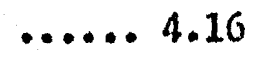

thus

$$
S^{t}=Q^{t} L^{t}
$$

(......4.17

Substituting 4.16 and 4.17 into 4.15 wa have

$$
2 V=p^{t} L \mathrm{~T} L
$$

.... 4.18

In nonal co-ordinates the potential energy may be expressed as

$$
2 V=Q^{t} n Q
$$

On comparing 4.18 with 4.19 we have

$$
\Lambda=L^{t} E L
$$

For one nomal vibrational frequency $\lambda_{k}$ the relation from 4.20 may be written as

$$
\lambda_{k}=\sum_{i, j} f_{i j{ }^{\ell} k^{\ell} j k}
$$

in wirich the potential energy is 


$$
V\left(\lambda_{k}\right)=1 Q^{2} k \sum_{i, j} f_{i j}{ }^{k} k^{k} j k
$$

where $f_{i j}{ }^{l} i k^{l} k_{k}$ is the potential enorgy distribution in each symetry co-ordinate. The tom is generally large when $1-j$, thich is quite useful for band assignants. For example, if this tern is oxceodingly large for $\mathrm{S}_{i}$, tilis nomal vibration can bo assigned to the group vibration represented by $S_{1}$. If boti $f_{i i^{2}}{ }^{2}$ and $f_{j j} \ell^{2}{ }_{j k}$ are relatively large comared with those of other syantry co-ordinates, the nomal vibration is assigned to a coupled vibration bstween those represented by symatry comordinatos $s_{i}$ and $s_{j}$. 


\section{CIAPTER 5}

\section{G-inTRX}

5.1 Construction of the G matrix

Gnerally the $G$ matrix in intemal co-ordinates can be constructed by the following steps:-

(a) Evaluate the matrix $B$ winch determines the contribution of eacil cartesian displacenent co-ordinate $q_{i}$, to the intemal co-ordinates $\Delta R_{t}$ ( $\Delta R$ is from now on being used in place of $R$ for the intemal displacement co-ordinate).

(b) Evaluate the $D$ natrix from

$$
D=B \mu^{2}
$$

where $\mu^{2}=$ diagonal matrix of square roots of reciprocal masses.

(c) The $G$ matrix in termis of internal co-ordinates can be evaluated fron

$$
G=D D^{t}
$$

\subsection{Evaluation of the $B$ matrix}

The $B$ matrix elezents are evaluated using Wilson's $S$ vector technique $(2,3)$ where the unit vectors $c_{i j}$ are assumed along bonds connecting atoms $i$ and $j$. Thus, for any bond between atom $i$ and $j$

$$
e_{\mathbf{i j}}=-e_{\mathbf{j i}}
$$


These bond vectors can easily be expressed in terms of cartesian displacement vectors on each atom. The novenent of each aton is described by a vector $S_{t_{i}}$, related to the bond vector $e_{i j}$.

1. Bond stretching co-orcinates ( $\triangle P$ )

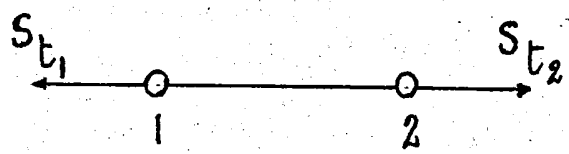

Fig. 2

Let $\Delta R$ be the increase in the distance batween atoms $I$ and

2. Cleariy

$$
\begin{aligned}
& s_{t_{2}}=e_{21}=-e_{12} \\
& s_{t_{2}}=e_{12}
\end{aligned}
$$

(..... 5.4

(..... 5.5

If these are expressed in cartesian displacenont vectors, the coefficient of $S_{t_{i}}$ will be the B matrix elenents associated with the co-ordinate $\mathrm{AR}$.

2. Valence angle bending co-ordinates

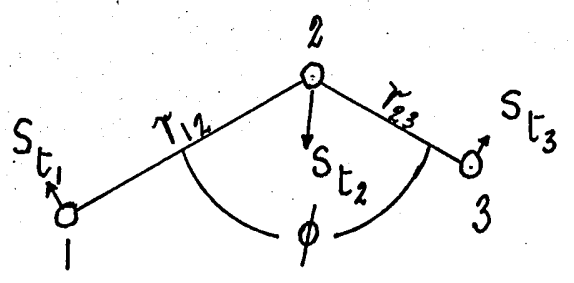

Eig. 3

Considor the bond system shown in Fig. 3 and defined by acons 1,2 and $3 . e_{21}$ and $e_{23}$ are unit vectors directed along 
the bonds $2-1$ and $2-3$ respectiveiy.

The $S_{\boldsymbol{t}_{\mathbf{i}}}$ vectors are given as follows:- .

$$
\begin{aligned}
& s_{t_{1}}=\frac{\cos e_{21}-e_{23}}{r_{12} \sin \phi} \\
& s_{t_{3}}=\frac{\cos \phi e_{23}-e_{22}}{r_{23} \sin \phi}
\end{aligned}
$$

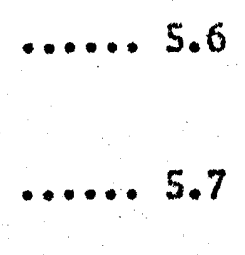

and $s_{t_{2}}=-s_{t_{1}}-s_{t_{3}}$

The coefficients of $S_{t_{i}}$ in terns of cartesian displacenent voctors are the $B$ matrix elements corresponding to the particular angle bending co-ordinate concerned.

3. Angle between a bosd and a plane defined by tiro bonds

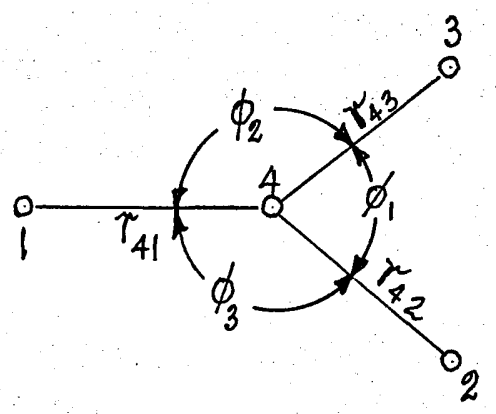

II. 4

This type of the co-ordinate is the angle forred by a band 4,1 and the plane of the three atoms 2,3 and 4, all four atoms being in one plane. For this situation the magnitudes of the vectors aro

$$
s_{t_{1}}=\frac{1}{r_{41}}
$$

"end aton" ...... 5.9 


$$
\begin{aligned}
& s_{t_{2}}=\frac{\sin \phi_{2}}{r_{42} \sin \phi_{1}} \\
& s_{t_{3}}=\frac{\sin \phi_{3}}{r_{43} \sin \phi_{1}} \\
& s_{t_{4}}=-s_{t_{1}}-s_{t_{2}}-s_{t_{3}}
\end{aligned}
$$

\section{Torsion}
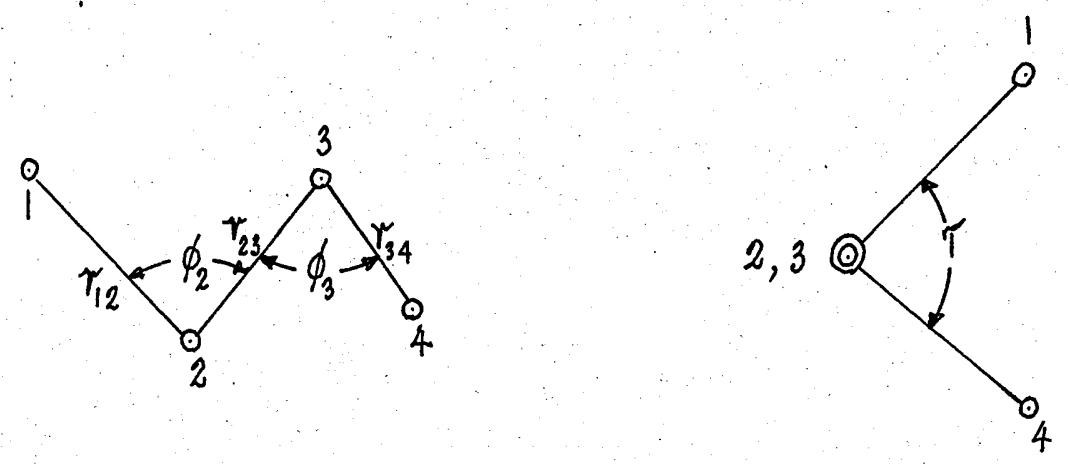

Fig. 5

The torsion angle $\Delta T$ is defined as the change from the equilibrium configuration in the angle between the planes dofined by the nuclei 1,2 and 3 and 2,3 and 4 . The $s_{t_{i}}$ vectors may be written as

$$
\begin{aligned}
& s_{t_{1}}=\frac{1}{r_{12} \sin \phi_{2}} \cdot \frac{e_{21} \times e_{23}}{\sin \phi_{2}} \\
& s_{t_{4}}=\frac{1}{r_{34} \sin \phi_{3}} \cdot \frac{e_{34} x e_{32}}{\sin \phi_{3}} \\
& s_{t_{2}}=-\frac{r_{23}-r_{12} \cos \phi_{2}}{r_{23} r_{12} \sin _{2}} \cdot \frac{e_{21} x e_{23}}{\sin \phi_{2}}-\frac{\cos \phi_{3}}{r_{23} \sin \phi_{3}} \cdot \frac{e_{34} x e_{32}}{\sin \phi_{3}} \\
& \text {..... } 5.15
\end{aligned}
$$




$$
s_{t_{3}}=-\frac{r_{23}-r_{34} \cos \phi_{3}}{r_{23} r_{34} \sin _{3}} \cdot \frac{e_{34} \times e_{32}}{\sin \phi_{3}}-\frac{\cos \phi_{2}}{r_{23} \sin _{2}} \cdot \frac{e_{21} \times e_{23}}{\sin \phi_{2}} \ldots .16
$$

NOTE

1. The sum of the $S_{t_{i}}$ vectors is zero.

2. AlI $S$ vector expressions are caken from Steele (2) and hilson, Decius and Cross (3). There is a sign error in the expression for $s_{t_{2}}$ in $(3 \mathrm{p} .61)$. The sign before the second tern should be and not -. 
CHAPTER 6

FORCE MIBLDS

\subsection{Force constants}

The vibration frequencies and the form of (3N-5) nonnal vibrations of an $\mathrm{N}$ atomic nolecule depends upon

(1) the atowic masses;

(2) geometrical distribution of the vibrating nuclei;

(3) the force field which tends to restore the molecule to its intomal equilibriun configuration during eny distortion.

The atonic masses and their geonecrical distribution must be imom before any force constant celculation can be attempted. The force field arises from the fact that the equilibriun positions of tho nuclei correspond to a minimum in the electronic energy of the molecule. Displacenent of the muclei causes a slight increase in the electronic energy.

lowever, in so far as one is concerned with the nomal state of the molecule, one of tire best representations of its condition is provided by the force field for the molecule; that 1s, a reprosentation of the forces that arise when the atons are displaced fron their equilibriun positions. This becones a detemination of the various 'force constants' for the bonds in the molecule and for the angular. Ioration of the atons. The force constant calculations heve also been used as supporting evidence for molecular geonatry. 
One should not attempt to define more force constants than there are frequencies available. This often means that all the terns in the general quadratic expression cannot be evaluated and the problem must be approximated by writing at lenst sone force constants equal to zero (5).

Practically, as an aproxination, a first set of force constants for a melecule nay be obtained from other molecules under the restrictions that

(1) They are of similar types (e.g. bond stretch + bond stretch, angle bending $\rightarrow$ angle bending, etc.).

(2) They are acting betteen the sane nuclei.

(3) They were calculated using the same type of force field.

In many cases thoy also be obtained by extrapolation of the sets of force constants of similer nolecules, e.g. a set of halogenated molecules.

\subsection{Force fiolds}

Athough the general valence force fiold is supposed to be the best force flicld to apply to the nolecule because it consists of all possible interactions betreen atons in a miecule, the number of forse constants required is moro than the number of frequencies available. This makes it difficult to apply to poiyatonic molecules.

As in the oxalic acia rolecule, for oxample, there are altogether 63 force constants (using 1st mode1 - see section 15.1, 16.2) in the potential field of the inplane vibrations and only 
13 inplane fundanantal frequencies. Thus, it is impossible to work out all the force constants. It is also very difficult to apply the modified valence force field because there are too many force constants to climinate, $0 . \mathrm{g}$. there are 50 force constants of the inplane vibrations of oxalic acid to eliminate in order to make the calculation possible.

Two force ficlds that are often appliod to polyatomic molecules are the valence force field and the Urey-Bradiey force fleld.

In the valence force field it is assured that

(a) there is a strong restoring force in the line of every valence bond if the distance of the two atoms bound by this bond is changed;

(b) there is also a restoring force opposing a change of the angle between two valence bonds.

The potential energy in the valence force field is a purely quadratic function of the changes of the valence bonds and valence angles and there is no cross tem in the potential energy. In many cases tinis force field failed to give a close tit of calculated to observed frequencies for polyatonic nolecules.

One of the most well lanow force fieldis for polyatomic molecules is the Urey-Bradley force field. In this force field the potential energy is expressed in terms of force constants and geointry. It is supposed to be botter tian the valence force field for the following reasons:-

(1) In Urey-ibradley force fields a large number of force constants is reduced systenatically and the potential 
energy not only includes the pure quadratic terms, but also sone cross terms.

(2) For many cases the Urey-Bradley force field gives a better fit of the calculated to the observed frequencies.

For nost polyatomic molecules the force constants have been calculated using the Urey-Bradley force field. Thus, transference and extrapolation of force constants anong nolecules can be made easier using this force field.

For the reasons nentioned above, the Urey-Bredley force field was selocted to aply to the oxalic acid molecule.

\subsection{Urey-Bradley force fiele}

In the Urey-Bradley force field the potential energy change due to a change in bond length $\left(\Delta r_{i}\right)$ and bond angle $\left(\Delta \alpha_{i}\right)$ can be expressed as:

$$
\begin{aligned}
V= & \sum\left[\mathrm{K}_{i} \mathrm{r}_{i 0}\left(\Delta \mathrm{r}_{i}\right)+\frac{1}{i} \mathrm{~K}_{i}\left(\Delta \mathrm{r}_{i}\right)^{2}\right] \\
& +\sum\left[\mathrm{H}_{i} \mathrm{r}_{i \alpha^{2}}\left(\Delta \alpha_{i}\right)+\mathrm{H}_{i} \mathrm{r}_{i a^{2}}{ }^{2}\left(\Delta \alpha_{i}\right)^{2}\right] \\
& +\sum\left[\mathrm{F}_{i} q_{i o}\left(\Delta q_{i}\right)+i \mathrm{~F}_{i}\left(\Delta q_{i}\right)^{2}\right]
\end{aligned}
$$

where $\Delta r_{1}=$ change in bond length

$\Delta \alpha_{i}=$ change in bond angle

$\Delta q_{i}=$ cinnge in distance between non bonded ators

$\mathrm{K}$ a stretching force constant

$H=$ bonding force constant

$F=$ repulsive force constant 
$r_{10}, r_{i \alpha}$ and $q_{i 0}$ are the equilibriun values of distances wich are inserted in order to nate all the force constants, $K_{i}$, $H_{i}^{\prime}, E_{i}, K_{i}, H_{i}$ and $F_{i}$ diminsionally similar.

Here $A q$ in the above expression needs to be elininated.

The following expressions for the $\Delta q$ 's are in (1), (13), (25).

There are two types of $\Delta \mathrm{q}_{\mathrm{ij}}$ :

(i) That where atoms $i$ and $j$ are bonded to a coranon atom

k. $\Delta q$ can be eliminated as follows:-

$$
\begin{aligned}
& \text { From } q_{i j}{ }^{2}=r_{i}^{2}+r_{j}^{2}-2 r_{i} r_{j} \cos \alpha_{i j} \\
& \left(q_{i j}+\Delta q_{i j}\right)^{2}=\left(r_{i}+\Delta r_{i}\right)^{2}+\left(r_{j}+\Delta r_{j}\right)^{2}-2\left(r_{i}+\Delta r_{i}\right)\left(r_{j}+\Delta r_{j}\right) \cos \left(\alpha_{i j}+\Delta \alpha_{i j}\right) \\
& =\left(r_{i}+\Delta r_{i}\right)^{2}+\left(r_{j}+\Delta r_{j}\right)^{2}-2\left(r_{i}+\Delta r_{i}\right)\left(r_{j}+\Delta r_{j}\right) \\
& \left(\cos \alpha_{i j} \cos \Delta \alpha_{i j}-\sin a_{i j} \sin \Delta \alpha_{i j}\right)
\end{aligned}
$$

in wich for a small angle chango

$$
\begin{aligned}
& \cos \left(\Delta \alpha_{i j}\right)=1-\Delta \alpha_{i j}{ }^{2 / 2} \\
& \sin \left(\Delta \alpha_{i j}\right)=\Delta \alpha_{i j}
\end{aligned}
$$

Then $\left(q_{i j}+\Delta q_{i j}\right)^{2}=\left(r_{i}+\Delta r_{i}\right)^{2}+\left(r_{j}+\Delta r_{j}\right)^{2}-2\left(r_{i}+\Delta r_{i}\right)\left(r_{j}+\Delta r_{j}\right)$

$$
\left\{\cos \alpha_{i j}\left(1-\Delta \alpha_{i j}{ }^{2 / 2}\right)-\sin \alpha_{i j} \cdot \Delta \alpha_{i j}\right\}
$$


Subtacting 6.2 fron 6.4 and then neglecting toms of higher than second order in snall quantities we have

$q_{i j} \Delta q_{i j}=\Delta r_{i}\left(r_{i}-r_{j} \cos \alpha_{i j}\right)+\Delta r_{j}\left(r_{j}-x_{i} \cos \alpha_{i j}\right)+r_{i} r_{j} \sin \alpha_{i j} \cdot \Delta \alpha_{i j}$

$+\left(\Delta r_{i}\right)^{2} / 2+\Delta r_{j}^{2} / 2-\Delta r_{i} \Delta r_{j} \cos \alpha_{i j}+\left(r_{i} \Delta r_{j}+r_{j} \Delta r_{i}\right) \Delta \alpha_{i j} \sin \alpha_{i j}$

$-\left(\Delta q_{i j}\right)^{2 / 2}+\left(r_{i} r_{j}\left(\Delta a_{i j}\right)^{2} / 2\right) \cdot \cos a_{i j}$

On squaring 6.5 , retaining terms only to the second order as before and substituting the resulting expression for $\left(\Delta q_{i j}\right)^{2}$ back into 6.4 we get

$$
\begin{aligned}
& \Delta q_{i j}=s_{i j}\left(\Delta r_{i}\right)+s_{j i}\left(\Delta r_{j}\right)+\left(t_{i j} t_{j i} r_{i} r_{j}\right)^{\frac{1}{2} \cdot \Delta a_{i j}}+\frac{2}{2 q_{i j}}\left\{t_{i j}{ }^{2}\left(\Delta r_{i}\right)^{2}\right. \\
& +t_{j i}{ }^{2}\left(\Delta r_{j}\right)^{2}-s_{i j} S_{j i} r_{i} r_{j}\left(\Delta \alpha_{i j}\right)^{2}-2 t_{i j} t_{j i} \Delta r_{i} \Delta r_{j}+2 t_{i j} s_{j i} r_{j} \Delta r_{i}: \\
& \left.\Delta \alpha_{i j}+2 t_{j i} s_{i j} r_{i} \Delta r_{j} \cdot \Delta \alpha_{i j}\right\}
\end{aligned}
$$

whero

$$
s_{i j}=\left(r_{i}-r_{j} \cos \alpha_{i j}\right) / a_{i j}
$$

and

$$
t_{i j}=r_{j} \sin a_{i j} / q_{i j}
$$

By substituting $\Delta \mathrm{q}$, the potentinl energy is then expressed in an independent set of co-ordinates. However, this substitution hes the effect of introducing the $F^{\prime}{ }^{\prime}{ }^{\prime}$ into the coefficients of the quadratic terns. The coefficients of $F_{i f}$ and $F_{i j}$ are shown in Table 1 below: 
Table 1

The coefficients of $F_{i j}$ and $F^{\prime}{ }_{i j}$ when atons $i$ and $j$ are bonded to a comon aton 1

\begin{tabular}{|c|c|c|}
\hline \& vector & $p_{i j}$ & $F_{i j}$ \\
\hline$\left(\Delta x_{i}\right)^{2}$ & $s_{i j}{ }^{2}$ & $t_{i j}{ }^{2}$ \\
\hline$\left(\Delta r_{j}\right)^{2}$ & $s_{j i}{ }^{2}$ & $t_{j i}{ }^{2}$ \\
\hline$\left(r_{i} \Delta \alpha_{i j}\right)^{2}$ & $t_{i j} t_{j i}\left(r_{j} / r_{i}\right)$ & $-s_{i j} s_{j i}\left(r_{j} / r_{i}\right)$ \\
\hline$\Delta \mathbf{r}_{\mathbf{i}} \Delta \mathbf{r}_{\mathbf{j}}$ & $S_{i j} S_{j i}$ & $-t_{i j} t_{j i}$ \\
\hline$\Delta x_{i}\left(x_{i} \Delta \alpha_{i j}\right)$ & $s_{i j} \sqrt{t_{i j} t_{j i}}\left(r_{j} / r_{i}\right)^{\frac{1}{2}}$ & $t_{i j} s_{j i}\left(r_{j} / r_{i}\right)$ \\
\hline$\Delta r_{j}\left(r_{i} \Delta \alpha_{i j}\right)$ & $S_{j i} \sqrt{t_{i j} t_{j i}}\left(r_{j} / r_{i}\right) l$ & $t_{i j} s_{i j}$ \\
\hline
\end{tabular}

(ii) Thut where atoms $i$ and $f$ are bonded respectively to two othor atoms $k$ and $l$ which are in tum connected by $a$ bond ke. The cosfficionts of $F_{i j}$ and $F_{i j}$ may be obtainea in a sinilar way and are shom in Table 2.

\section{Table 2}

The coefficients of $F_{i j}$ and $F_{i j}$ when atons $i$ and $j$ are bonded to two other atoms $k$ and $\ell$ which are in tum connected by a bond ke

\begin{tabular}{|l|l|l|}
\hline f vector & $F_{i j}$ & $F_{i j}^{\prime}$ \\
\hline$\left(\Delta r_{i}\right)^{2}$ & $v_{i j}{ }^{2}$ & $\left(1-v_{i j}{ }^{2}\right)$ \\
$\left(\Delta r_{j}\right)^{2}$ & $v_{j i}{ }^{2}$ & $\left(1-v_{j i}{ }^{2}\right)$ \\
$\left(\Delta R_{k \ell}\right)^{2}$ & $a_{i j}{ }^{2}$ & $b_{i j}{ }^{2}$ \\
\hline
\end{tabular}




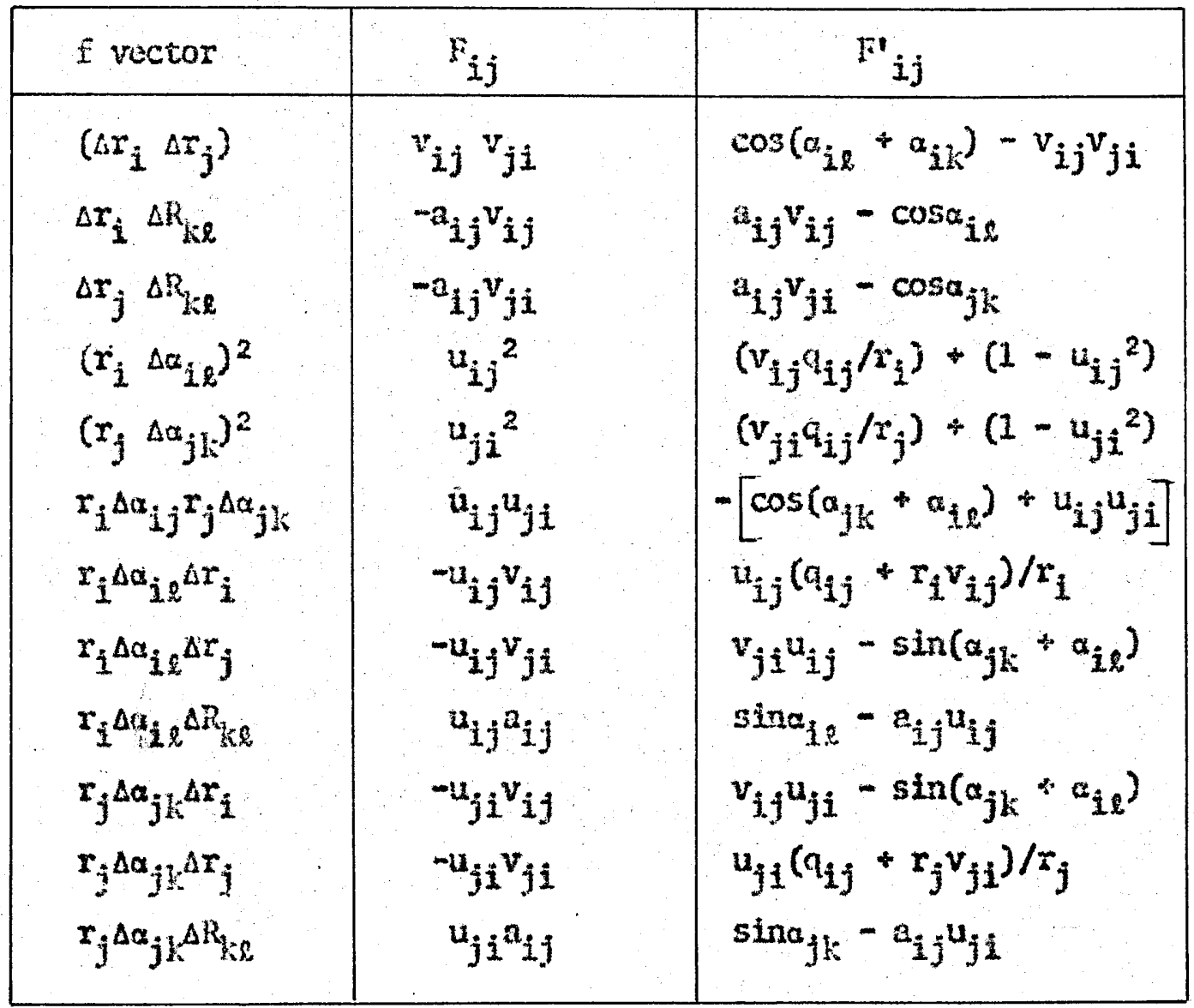

in which $a_{i j}-\left(R_{k t}-r_{i} \cos \alpha_{i 2}-r_{j} \cos \alpha_{j k}\right) / a_{i j}$

$$
\begin{aligned}
& b_{i j}=\left(x_{i} \sin \alpha_{i l}-x_{j} \sin \alpha_{j k}\right) / a_{i j} \\
& u_{i j}=a_{i j} \sin \alpha_{i \ell}+b_{i j} \cos a_{i k} \\
& u_{j i}=a_{i j} \sin \alpha_{j k}-b_{i j} \cos \alpha_{j l} \\
& v_{i j}=a_{i j} \cos \alpha_{i \ell}-b_{i j} \sin \alpha_{i l} \\
& v_{j i}=a_{i j} \cos \alpha_{j k}+b_{i j} \sin \alpha_{j l}
\end{aligned}
$$




\section{CIAPTER 7}

ForCe CONSTATE CuCULATONS

\subsection{Nethod of trial and error}

The main part of the calculation in vibrational problens is to find a gooi set of force constants in a specific force field Which will give calculated frequencies fitting well to the observed values. Thus, the observed values and their assignnents must be obtained before a calculation is perforned. The mothod of trial and error nay be applied by first guessing a set of force constants and thon calculating the freguencies. If both observed and calculated values do not agree well, that set of force constants is modifled. The process is repated until a satisfactory set of force constants is formd.

Gonerally the vibrational frequencies are calculated using the secular equation

$$
|g \mathrm{D}-I \lambda|=0
$$

If $G$ and $D$ are expressed in synmetry co-orainates, the secular determinant nay be factorised into several smaller blocks. Each block belongs to a species and each species can bo solved separately. In this way the calculation for a reoleculo of high synotry can be solved more easily.

In the case of oxalic acid the number of independent force constants required by the urey-bradley foree field to describe the 13 inplane vibrations is 13 . Thoy are onitnarily found by trial 
and orror methods and nay bo judged acceptable if they appear to be reasonble in magnitudo and if the nomal frequencies to which they lead are in good agreemont with the observed fundanontals. The inplane vibrational frequencios of oxalic acid can be calculeted from tise $13 \times 13$ matrix which contains two smaller matrices. A $7 \times 7$ matrix belongs to the Ag species and a $6 \times 6$ matrix belongs to the Bu species. Each of these is too large to be solved by hand. With tho aid of tine computer it is possible to carry out the calculation without difficulty.

7.2 Hethod of least squares refinement (24)

Goneraliy the first set of force constants hay be obtained by guessing or transferring the force constants fron another wolecuie. The refinement of this approximate set of force constants will be porfomed by trial and error. The criterion for this is that the percontage discrepancy betweon calculated and observed frequencies should be as snall as possible with the largest discropancy not greater than $5 \%$.

A further systenatic refinomant of force constants may be obtained by the least squares mothod. Whis mothod is used to adjust or refine the force constants in accordance with a prescribed and definite mathenatical process. This mathod is very convenieit because it can be easily adapted for matrix operation.

It is supposed that a set of d experimental froguencies $\left(v_{0}\right)$ is aveilable and the problen imolves $r$ indepencent force constants 
in which $d \geqslant r$. Here our rumpse is to aljust the force constents weil the calculated frequencies are fitted to the observod froquancios: in other words, co got the minimu variance. Ideally for the best fit the variance will becons zero, this will not be so because of experinatal error and the epproximate force field used.

We define the neasure of fit or the variance as

$$
s^{i-1}=\sum_{n=1}^{d} m_{n}\left(v_{n}^{i}-v_{n}^{9}\right)^{2}
$$

where $n=1,2, \ldots \ldots d$

$\nu_{n}^{0}=$ observed frequencies

$\nu_{n}{ }^{i}=$ calculatod frequancies whoro tho index i labels the stage of calculation and hay have the value $1,2,3, \ldots$

$\mathrm{w}_{\mathrm{n}}=$ weighting factors

The frequencios calculated from the ith set of force constants are indicatod by $v^{i}$. The differences between the frequencies of the $(\mathfrak{i}+1)$ th and ith sets are given by

$$
\Delta v_{n}^{i}=v_{n}^{i+1}-v_{n}^{i}
$$

A corrosponding expresston

$$
\Delta k_{n}^{i}=k_{m}^{i+1}-k_{n}^{i}
$$

applies to the adjustments in the force constants. The new variance beconses 


$$
s^{i}=\sum_{n=1}^{d} w_{n}\left(v_{n}^{i+1}-v_{n}{ }^{0}\right)^{2}
$$

Applying 7.3 the equation 7.5 becomes

$$
s^{i}=\sum_{n=1}^{i} v_{n}\left(v_{n}^{i}+\Delta v_{n}^{i}-v_{n}^{0}\right)^{2}
$$

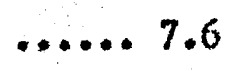

In matrix notation oquation 7.6 can be written as

$$
s^{i}=\left[v^{i}+\Delta v^{i}-v^{0}\right] \mathfrak{t} n\left[v^{i}+\Delta v^{i}-v^{0}\right]
$$

whore the brackets denote a colum or row matrix. $\mathrm{W}$ is a diagonal

natrix whose elcments represent the statistical weights of the observed frequencios.

If $w$ is taken as $1 / v$, then the best fit will be on ax cbsolute basis in the frequencies. On the other land, if if is taken as $1 / v^{2}$, the bese fit will be a percentage basis in the frequencios.

A rolation betrien $\Delta v^{i}$ and $\Delta k^{i}$ is assumed by

$$
\Delta v^{2}=J \Delta k^{1}
$$

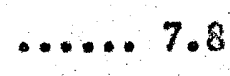

whore $\mathrm{J}$, the Jacobian matrix, is defined with olemonts

$$
J_{n \pi}^{i}=\frac{\delta v_{m}^{i}}{\partial x_{m}^{i}}
$$

and dinension $d x r$. 
Sustituting 7.8 in 7.7 and collecting the terns, the expression becomes

$$
\begin{aligned}
s^{i}= & {\left[v^{i}-v^{0}\right] t\left[v^{i}-v^{0}\right]+2\left[v^{i}-v^{0}\right] t w J^{i}\left[\Delta k^{i}\right] } \\
& +\left[\Delta k^{i}\right] \mathrm{t}_{j i} t_{\| J^{i}}\left[\Delta k^{i}\right]
\end{aligned}
$$

If $\mathrm{s}^{\mathrm{i}}$ is nininum

$$
\frac{\delta S^{i}}{\delta \Delta k^{i}}=0=2\left[v^{i}-v^{o}\right]_{t} w J^{i}+2\left[\Delta k^{i}\right]^{t} J^{i t} w^{i}
$$

Transposing tile tems we have

$$
\Delta K^{i}=\left(J^{i} t_{W J^{i}}\right)^{-1} J^{i t} W\left[x^{i}\right]
$$

where $\left[x^{i}\right]=\left[v^{i}-v^{0}\right]$

Then $\left[\Delta k^{i}\right]$ values deternined from 7.12 are used to form a new set of force constants.

The vector $\left[x^{i}\right]$ is ecsily constructed from the differences between the experimental frequencios and the calculated frequencios fron the ith set of force constants.

The elements of the dingeanl satrix are usually taken to be $1 /\left(v_{n}^{0}\right)^{2}$ in order to ensure that the frequencies are fitted on e porcentage rather than an absolute basis.

7.3 Method of obtaining the Jacobian natrix for the least squares method

There are two metiods of deterinining the Jacobian elements. 1st method

The first nothod was suggosted by Mann, Shinanouchi, Meal and 
Fano (24). They calculated the Jacobien matrix by making a successive variation of each force constant in tum, followed by repeated calculation of the frequencies. In this method it is necossary that the force constants of the first set are not very far from the final values. This noans that the adjustronts are in the nature of refinenents rather than gross changes. The Jacobian natrix is constructed in the following way.

Let the Eirst set of force constants be the ith set. The $(i+1)$ th set of force constants can be obtained by giving a small increnent $0.01 \mathrm{~m} z / \mathrm{A}$ to one of the $\mathrm{r}$ force constants. Both sets are then used to calculate the $s$ fundamental frequencies. Thus, a colum of the Jacobian matrix is obtained by

$$
[J]=\frac{\Delta v_{n}^{i}}{\Delta k}=\frac{v_{n}^{i+1}-v_{n}^{i}}{\Delta k}
$$

in which $\Delta \mathrm{k}=0.01 \mathrm{md} / \mathrm{A}$.

The same incremant is then given to each of the other force constants in turn. In this way all the $r \times s$ elonents of the Jacobian matrix are obtained.

2nil nethod

The second method is described in Hilson, Decius and Cross (3), Overend and Seinerer (25), thills (68), and Steele (1).

This rathod gives the Jacobian eletwents in tems of the latent vectors $L$ of the secular equation $|G F-I \lambda| L=0$. As mentioned bofore in equation 4.9 and 4.10

$$
G=L^{2}
$$




$$
L_{\text {FL }}=\Lambda
$$

It is assumed that the change in the latent vectors are insignificant for small changes in the calculated frequencies, that is, $\Delta v$ is quite small. A small correction $\Delta F$ is required to reduce the error in the calculated frequencies.

Let the error be given by

$$
v_{c a l}-v_{\text {obs }}=\Delta v
$$

If we define

$$
H=L^{t} \Delta F
$$

the secular deterninant for the purturoed problem would have the form

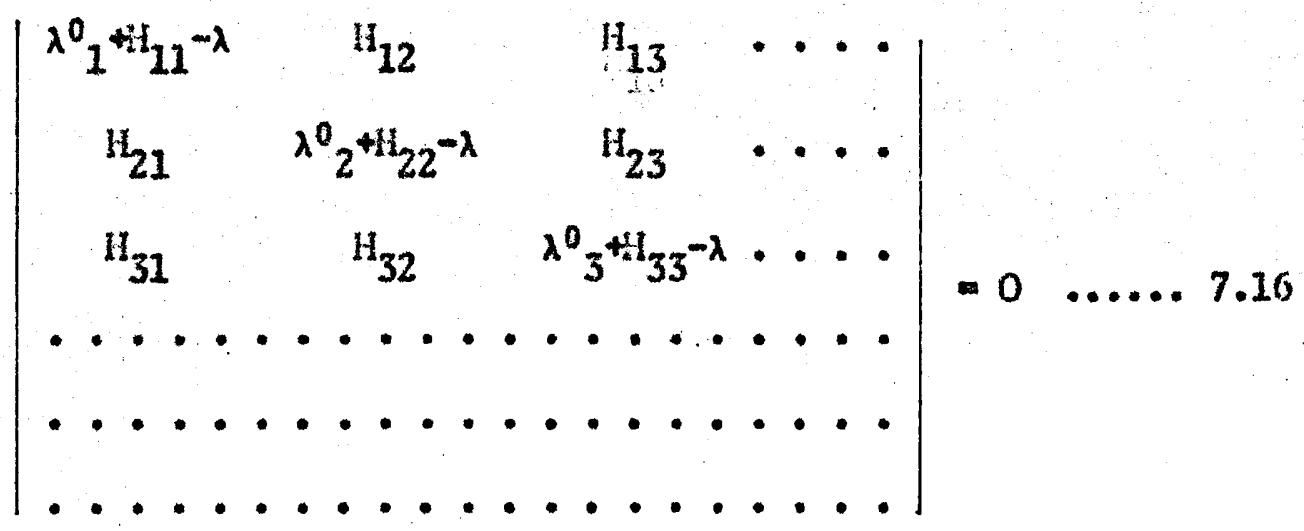


The first order approximation to this would give the nth root as

$$
\lambda_{n}=\lambda_{n}^{0}+H_{t t}
$$

Substituting 7.15 into 7.17 we have

$$
\lambda_{n}=\lambda_{n}^{0}+\sum_{t^{\prime} t^{\prime \prime}} e_{t^{\prime} n^{2} t^{\prime \prime} n^{\Delta F} t^{\prime} t^{\prime \prime}}
$$

or

$$
\Delta \lambda_{n}=\sum_{t^{\prime} t^{\prime \prime}} e_{t} n^{2} t^{\prime \prime} \Delta^{\prime} F_{t} t^{\prime \prime}
$$

If we define $\mathrm{J}$ as

$$
J=\frac{\delta \lambda_{n}}{\delta t^{\prime} t^{\prime \prime}}
$$

we have

$$
\frac{\delta \lambda_{n}}{\delta F_{t^{\prime} t^{\prime \prime}}}=2 \varepsilon_{t^{\prime} n^{2} t^{\prime \prime} n}
$$

and

$$
\frac{\delta \lambda_{n}}{\delta F_{t}^{\prime} t^{\prime}}-e_{t n^{\prime} t^{\prime} n}
$$

where $t$ and $t^{\prime \prime}$ denote row and colum numbers in $F$, and $t_{t} n$ is the entry in the nth row of $L_{h}$.

Thus one row of the Jacobian natrix can be obtained by combinations of $l_{n}$ elements, where $l_{n}$ is the unpurturbed vectors corresponding to the nth root. The complete Jacobian can be 
canstructed by taking combinations of elements of all $\ell$ vectors by using equations 7.19 and 7.20 .

\subsection{A problem in the least squares method}

Although the least squares method provides a convenient methenatical expression for a computer to handle, and also provides a new set of calculated frequencies which are quite close to the observed frequencies, there are still sone difficulties that nay occur in the calculation.

(a) It was assuned that the relationship botween $\Delta \lambda$ and $\Delta \mathrm{K}$ is linear

$$
\Delta \lambda=J \Delta k
$$

This is not true in most cases and if the initial force constants are bad guesses, a bad fit will be obtained in the calculation.

(b) Singularity of the matrix ( $\mathrm{J}^{t_{\mathrm{W}}}$ ) is the other najor problem. In the least squares method the force constant refinements are performed using the expression.

$$
\Delta k=\left(J^{t}+J\right)^{-1} J t h[x]
$$

The main part is the term ( $\mathrm{J}^{\mathrm{t}}$ WJ). If this matrix is singular, that is, $\left|\mathrm{J}_{\mathrm{H}} \mathrm{J}\right|=0$, the problem is unrosolvable. (This maans that there is a set or sets of force constants with can be varied without affecting the vector $\mathrm{J}^{t}[\mathrm{X}]$ ). When the matrix $\left(\mathrm{J}^{t_{\mathrm{WJ}}}\right.$ ) is nearly singular, orrors ray occur in taking its inverse, causing corresponding errors in the calculated force constent 
correction ( $\Delta \mathrm{k})$. This also implies that the original $\mathrm{J}=\Delta \lambda / \Delta \mathrm{k}$ is ill conditioned, in other words the initial data do not suffice to fix the force fiold. The degree of singularity varies with the choice of the initial sot of force constants.

It is possible to check how close the matrix is to singularity by looking at the product of its diagonal elements. If the product of its diagonal elexpnts is much greater than the value of its deteminant the natrix is close to being singular. If the two values are close together, the natrix is nonsingular (123).

7.5 The uncertainties in the calculation

Uncertainties in the calculatian may occur for the following reasons:-

(1) The uncertainty caused by the experinental errors in neasuring the frequencies which usually are only a fow wave numbers from the true values.

(2) The force field is only approxinate, and so there must be a related uncertainty in the calculated frequencies.

(3) The use of an hamonic force field to fit observed frequencies which have not been corrected for anharmonicity.

(4) The uncertainty caused by the assumption of linearity in the relationship botween $\Delta \lambda$ and $\Delta k$. 
CHAPTER 8

INFRARED TRANSITIONS

\subsection{Hamonic vibration selection rules}

A transition with emission or absorption of radiation can occur between the vibrational states $v^{\prime}$ and $v^{\prime \prime}$ if one or more of

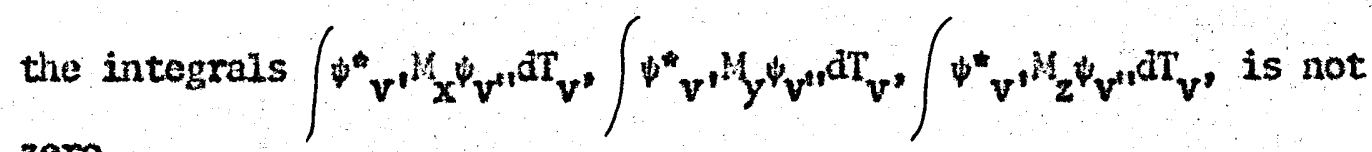
zero.

In general the electric moment is not necessarily a linear function of the co-ordinates of the atons, it can be expanded as a power series in the nomal co-ordinates (2), (3).

$$
M_{x}=M_{x}^{0}+\sum_{k=1}^{3 N-6}\left(\frac{\delta M}{\delta Q_{k}}\right)_{0} Q_{k}+\text { higher terms }
$$

with sinilar equations describing $\mathrm{M}_{\mathrm{y}}$ and $\mathrm{H}_{\mathrm{z}}$.

For the harinonic oscillator model the higher terms are assumed to be relatively small. Thus, $M$ can be written as

$$
M=N^{P}+\sum_{k=1}^{3 N-6}\left(\frac{\delta M}{\delta Q_{k}}\right)_{0} Q_{k}
$$

In order that a vibrating mode shall interact with radiation it is necessary that the dipole moment should change during the vibration, 1.e. $\frac{8 \mathrm{~K}}{8 \mathrm{Q}_{\mathrm{k}}} \neq 0$. This condition will hold if at least one of the components $\frac{\delta M_{x}}{\delta Q_{k}} \cdot \frac{\delta M_{y}}{\delta Q_{k}}$, or $\frac{\delta M_{z}}{\delta Q_{k}}$ along the co-ordinate axes changes during the vibration. The integral for $\mathrm{M}_{x}$ becomes 


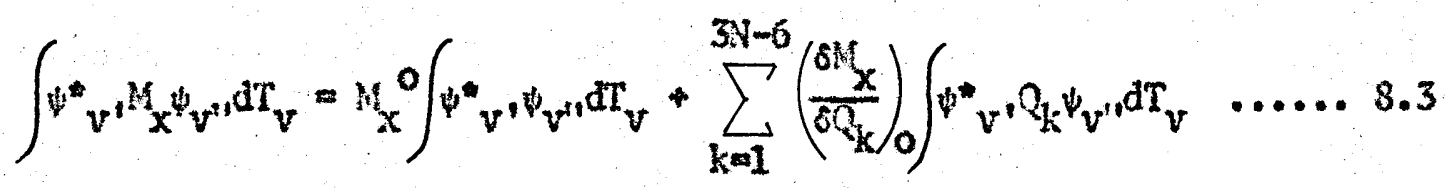

The first tem vanishes unless $v^{\prime}=v^{\prime \prime}$ because of orthogonality of the functions $\psi_{v}$. Therefore the permanent electric moment $s^{P}$ has no influence on the intensity of the vibrational transitions. The integral in the second term cen be split into factors as shown below:-

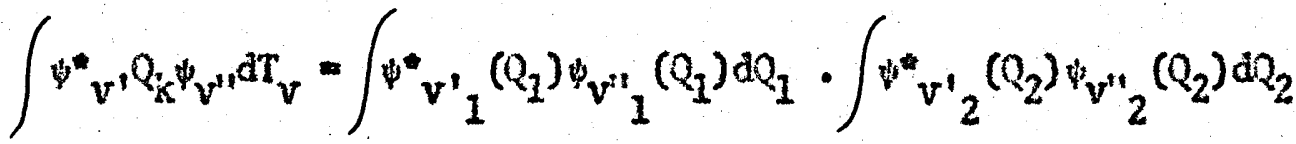

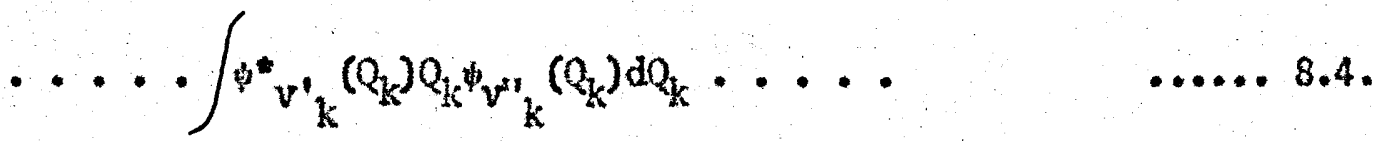

Because of the orthogonality of the functions $\varphi_{v}(Q)$ the integral will vanish unless $v_{1}^{\prime}=v_{1}^{\prime \prime}, v_{2}^{\prime} v_{2}^{\prime \prime}, \ldots$ etc., with the exception of $v_{k}^{\prime}$ and $v^{\prime \prime}{ }_{k}$. For these quantum numbers, it nust be true that $v_{k}^{\prime \prime}-v_{k}^{\prime}+1$ or $v_{k}^{\prime}-1$ if the factor $\int \psi^{*} v_{k}^{\prime}\left(Q_{k}\right) Q_{k}^{*} v_{k}^{\prime \prime}\left(Q_{k}\right) d Q_{k}$ is to be different fron zero (3 pp 42 43).

So on the basis of an harmonic oscillator model the only vibrational transitions which occur with the emission or absorption or radiation are those in which only one quantum number is changed by one unit only. That is $\sum \Delta v= \pm 1$.

\subsection{Anharmonicity}

On the basis of the hamonic oscillator nodel only fundamentals should appear in the absorption or emission spectra. Not 
all fundanentals are allowed but only those associated with normal modes of vibration which cause a change in the electric dipole moment of the nolecule.

Experimentally, i.e. for some nolecules, it is found that not only fundamentals appear, but also overtones and conbinations with relatively weaker intensities. It is certain from the evidence that the haxwonic oscillator approximation is not perfect, the actual behaviour of the particles is anharmonic.

There are two types of anhamonicity:

(1) Mechanical anharmonicity: This is dealing with the anharwonicity in the potential function. That is, the cubic, quartic and higher terms in the potential energy expression

$$
v=1 \sum_{j, k}^{3 N-6}\left(\frac{8^{2} V}{8 Q_{j} \delta Q_{k}}\right) Q_{j} Q_{k}+\text { higher terms } \ldots \ldots 8.5
$$

must be considered. These terns give rise to the occurdnce of the overtones and combinations. So there must be a snoll correction to the frequencies for the anarmonicity. The factor itself could be of much interest in many cases, and correction for it will normally nake only small chmges of the order of a few per cent in the force constants (5).

(2) Electrical anhamonicity: This type of anharminicity is concerned with the dipole monent expression.

$$
M=M^{0}+\sum_{k}^{3 N-6}\left(\frac{\delta M}{\delta Q_{k}}\right)_{0} Q_{k}+\text { higher terns } \quad \ldots \ldots 8.6
$$

Those higher terms also cause overtones and combinations to occur. 
Thus, they should not be ignored.

Due to the anharmonic nature of vibration the hamonic selection rule, $\Delta v= \pm 1$, no longer holds, that is

$$
\sum \Delta v= \pm 1, \pm 2, \pm 3, \ldots \ldots \pm n
$$

and such overtones as appear may not be exactly integral multiples of the fundanental frequencies. Transitions which correspond to sraller value of $\sum \Delta v$ are generally more intense.

In order to be able to nake a small correction for anharmonicity, it is necessary to have information not only on fundamontals, but also on overtane, combination and difference bands. Unfortunately, there is a great dearth of information on anharmonicity and only for a very linited number of polyatonic molccules can this correction be made. As for oxalic acid the information is not enough to deal with anhamonicity wo will assume that the particles behave as hamonic oscillators and neglect the small anount of correction in the frequencies for anhamonicity. 


\section{CIAPTER 9}

RAMAN TRANSITIONS

\subsection{Raman polarizability tensor}

A Ranan transition can occur only if the amplitude of the dipole monent induced by the incident radiation changes during the vibration considered.

For an isotropic molecule the magnitude of the induced dipole moment $P$ is given by

$$
p=a E
$$

where $\alpha$ is the polarizability and $E$ is the electric vector of the incident radiation of froquency $v$.

In general, however, for an anisotropic molecule equation 2.1 nust be replaced by the complote expression

$$
\begin{aligned}
& p_{x}=a_{x x} E_{x}+a_{x y} E_{y}+\alpha_{x z} E_{z} \\
& p_{y}=a_{y x} E_{x}+a_{y y} E_{y}+\alpha_{y z} E_{z} \\
& p_{z}=a_{z x} E_{x}+a_{z y} E_{y}+a_{z z} E_{z}
\end{aligned}
$$

in which the quantitios $\alpha_{\mathrm{FF}}$, are independent of the components of the electric vector, but are dependent upon the orientation of the nolecule relative to the non-rotating axes $x, y, z$. The quantities $a_{\mathrm{FF}}$ are called the components of a tonsor by virtue of their transformation under changes of the co-ordinate system (2), (3). 
In general the direction of the induced dipole is not parallel to the direction of the incident electric field vector. It can be shom, however, that a set of axes in the molecule exists such that the relation between $P$ and $E$ when referred to these axes, assumes the simple fom

$$
\begin{aligned}
& p_{1}=a_{1} E_{1} \\
& p_{2}=a_{2} E_{2} \\
& p_{3}=c_{3} E_{3}
\end{aligned}
$$

Such axes are called the principal axes of polarizability and the associated $\alpha_{i}, i=1,2,3$, the principal values of the polarizability. For an isotropic case

$$
a_{1}=a_{2}=\alpha_{3}=\alpha
$$

\subsection{Expansion of the polarizability in the nomal co-ordinntes}

The components of the polarizability have been assumed to be independont of the time. For a molecule executing small vibrations the polarizability may be expanded in terns of the nornal co-ordinates

$$
\alpha_{i}=\alpha_{i}^{0}+\sum_{k}\left(\frac{\delta \alpha_{i}}{\delta Q_{k}}\right)_{0} Q_{k}+\text { higher terns }
$$

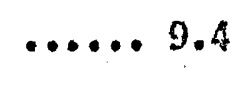

where

$$
Q_{k}=Q_{k}^{\circ} \cos \left(2 \pi v_{k} t+\varepsilon\right)
$$

in which $Q_{k}^{\circ}$ is the anditude of the kth nomal co-ordinate, 
$v_{k}$ is the associated frequency and $\varepsilon$ is the phase angle.

on applying equations 9.4 and 9.5 together with the

expression for the oscillating electric vector

$$
\begin{array}{rr}
E_{i}=E_{0 i} \cos 2 \pi v_{0} t & \ldots \ldots 9.6 \\
\text { into } P_{i}=\alpha_{i} E_{i} & \ldots \ldots .9 .7
\end{array}
$$

the corresponding component of the oscillating induced dipole

moment $p_{i}$ would, therufore, be of the form

$$
\begin{aligned}
P_{i}= & E_{o i}\left[a_{i}^{0} \cos 2 \pi v_{o} t+\sum_{k}\left(\frac{\delta a_{i}}{\delta Q_{k}}\right)_{0} Q_{k}{ }^{0}\left(1 \cos 2 \pi\left(v_{0}+v_{k}\right) t\right.\right. \\
& \left.\left.+\frac{1}{d} \cos 2 \pi\left(v_{0}-v_{k}\right) t\right\}+ \text { higher terms }\right] \quad \ldots \ldots 9.8
\end{aligned}
$$

Here $v_{0}$ is the incident or the Rayleigh Erequency and $v_{0} \pm v_{k}$ is the Raman frowuency.

The higher terns in the polarizability expansion leads to overtones and combinations, in a fashion similar to the infrared transitions. 


\subsection{Degree of depolarization}

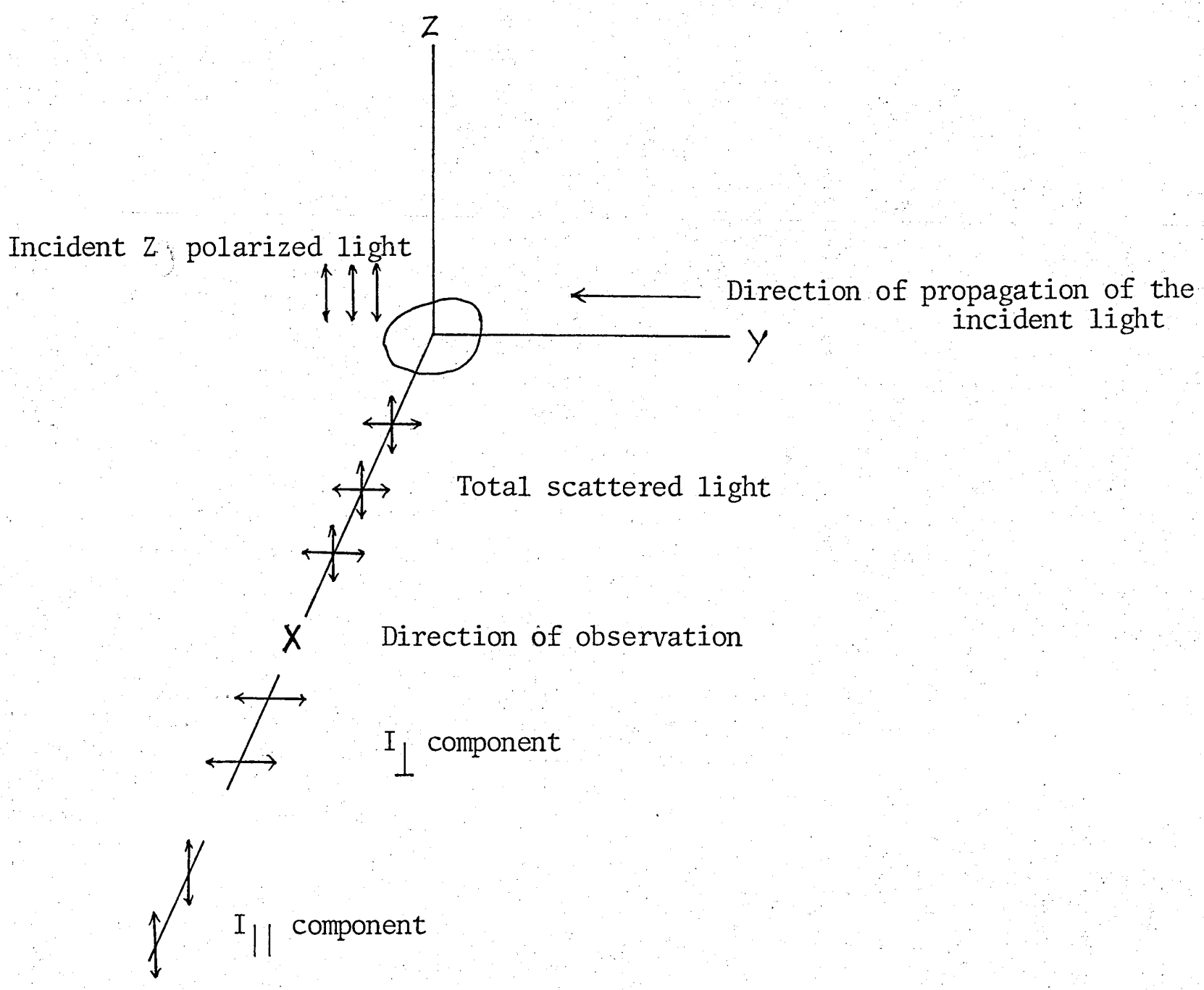

Fig. 6

Scheatic representation of exporinental conditions

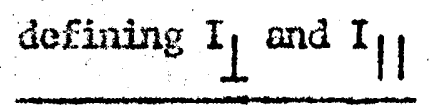

Here the $y$ axis is taken in the direction of propagation of the incident light, $z$ is the direction of the electric vector $\mathrm{E}$ of the incident radiation, and $x$ is the direction of the observation. 
The degree of depolarization $p$ is defined as the ratio of the intensity of the scattered light polarized perpendicular to the incideat electric vector $E$, called $I_{L}$, to that polarized parallel to $\mathrm{E}$, called $\mathrm{I}{ }^{\circ}$

$$
0=\frac{I}{I_{I}}
$$<smiles>C[13CH][13CH]</smiles>

In liquid and gas phases the polarizability can be averaged over all orientations of the system $(3$ p. 46$)$. Thus, when the linear polarized incident light is used and the observations are nade in a direction perpendicular to the electric vector $E$, the degree of depolarization is

$$
\rho_{\ell}=\frac{3 \beta^{2}}{45 \alpha^{2}+4 \beta^{2}}
$$

where $\alpha$ is the spherical part of polarizability and $\beta$ is the anisotropic part, which are defined by

$$
\begin{aligned}
& \alpha=\frac{1}{3}\left(\alpha_{1}+\alpha_{2}+\alpha_{3}\right) \\
& \beta^{2}=\frac{1}{2}\left[\left(\alpha_{1}-\alpha_{2}\right)^{2}+\left(\alpha_{2}-\alpha_{3}\right)^{2}+\left(\alpha_{3}-\alpha_{1}\right)^{2}\right]
\end{aligned}
$$

If $B=0, p_{2}=0$

If $\alpha=0$, and $\beta \neq 0, \rho_{\ell}^{\max }=\frac{3}{4}$ 
Thus, for a linear polarized incident bean when the degree of depolarization is $\frac{3}{4}$, it is called depolarized. If the degree of depolarization is sraller than $\frac{3}{4}$, it is satd to be polarized (partly or completely).

Moasurements of the degree of depolarization are very useful for the assignment of the Raman bends. This is because only the Paman bands corresponding to totally symetric vibrations can have a degree of depolarization smaller than $\frac{3}{4}$, that is, can be polarized (2).

\subsection{Raman selection rules}

A transition in the Ranan spectrm can occur between two vibrational states $v^{\prime}$ and $v^{\prime \prime}$ only if at least one of the integrals

$$
\begin{aligned}
& \int v^{*} v^{*} P_{X^{\psi} v^{\prime}} d T_{v} \\
& \int \psi^{*} v^{\prime \prime} y^{*} v^{\prime} d r_{v} \\
& \int v^{*} v^{\prime \prime} \mathrm{P}_{z^{\prime \prime}} \cdot d T
\end{aligned}
$$

is not zero where $v^{\prime \prime}$ and $v^{\prime}$ represent lower and upper vibrational states, $\psi^{*} v^{\prime \prime}$ represents the complex conjugate of the vibrational wave function of the lower state. $P_{x}, P_{y}$ and $P_{z}$ are the components of the induced dipole moment along $x, y$ and $z$ axos respectively. Each of the components of tho induced dipole monent depends not only on one corponent of the incident cloctric vector but on all $x, y$ and $z$ components as shom in equation 9.2 .

Every one of the six components of polarizability in general changes whei the nuclei are displaced fros? their equilibriun 
positions. For smaIl displacencnts the polarizability can be expmed as

$$
a_{x x}+\alpha_{x x}^{0}+\left(\frac{\delta a x x}{\delta Q_{i}}\right)_{0} Q_{i}+\text { higher terns }
$$

sinilarly for $\alpha_{y y}, c_{z z}, a_{x y}, \alpha_{x z} \& \alpha_{y z}$. The incident electric vectors nay be iritten as

$$
\begin{aligned}
& E_{x}=E^{\circ} \cos 2 \pi v t \\
& E_{y}=E_{y}^{\circ} \cos 2 \pi v t \\
& B_{z}=E_{z}^{\circ} \cos 2 \pi v t
\end{aligned}
$$

On applying equations 0.14 and 9.15 to equation 9.2 , it becomes

$$
\begin{aligned}
& p_{x}=\left(\alpha_{x x} E_{x}^{0}+\alpha_{x y} E_{y}^{0}+a_{x z^{2}}{ }_{z}\right) \cos 2 \pi v t+\sum\left[\left(\frac{\delta \alpha x}{\delta C_{i}}\right)_{0} z_{x}^{0}\right. \\
& \left.\left(\frac{\delta \alpha_{x y}}{\delta Q_{i}}\right)_{0} E^{0} y+\left(\frac{\delta \alpha_{x z}}{\delta Q_{i}}\right)_{0} E^{0} z\right] Q_{i}^{0} x:\left[\cos 2 \pi\left(\nu+v_{i}\right) t+\right. \\
& \left.\cos 2 \pi\left(v-v_{i}\right) t\right]+ \text { higher terns }
\end{aligned}
$$

and similarly for $\mathrm{P}_{y}$ and $\mathrm{P}_{z}$. That is, the induced dipole moment oscillates with the frequency $v$ of the incident radiation leading to Rayleigh scattering, and with froquency $v \pm v_{i}$, leasing to the Ranari scattering. Thus, a transition will appear in the Raman spectrute if and only if at least one of the six components of the change of polarizability $\left(\frac{\delta a_{x x}}{\delta y_{i}}\right)_{0},\left(\frac{\delta a_{y y}}{\delta Q_{i}}\right)_{0},\left(\frac{\delta a_{z z}}{\delta Q_{i}}\right)_{0},\left(\frac{\delta \alpha_{x y}}{\delta Q_{i}}\right)_{0},\left(\frac{\delta a_{x z}}{\delta a_{i}}\right)_{0}$ and $\left(\frac{b a y z}{\delta Q_{i}}\right)_{0}$ is different frot zero 
As in intrared, if we assume that the molecuie behaves as an hamonic oscillator, the solection rule is

$$
\Delta V= \pm 1
$$

That is, only fundamentals are allowed.

Experimentally it is found that the fundementals aro usually most intense but other transitions such as overtones and coabinations also appeor. This suggests that the hamonic oscillator approximetion is not porfect, the actual bohaviour of the molecule is anhamonic.

As in infrared spectra, both mechanical and eloctrical anhamonicity account for overtone and combination bands. The anhamonic selection mule is

$$
\Delta v= \pm 1, \pm 2, \cdots \cdots
$$




\section{CAapter 10}

ENBRGY LEVELS OF ASTMASTRTC TOP MOLECULES

10.1 Rotational energy levels of asymetric top molecules

According to quantur nechanics, the energy levels of asymetric top nolecules cannot be ropresented by an explicit formula analogous to that for the symetric top. There are $J+1$ sublevels of different energy for a symatric top molecule for cach value of $\mathrm{J}$, nately those with $\mathrm{K}=0,1,2 \ldots \mathrm{J}$, of which all but one $(h=0)$ are doubly degenerate with respect to the tirections of rotation. For an asynetric top moleculo this degeneracy is removed, thus, for each value of $\mathrm{J}$ there are $2 \mathrm{~J}+1$ different energy levels. This splitting may be called $k$-type doubling. Each energy level is distinguished by a subscript $T$ added to $\mathrm{J}$ suci that $\mathrm{T}$ takes tho values

$$
T=-J,-J+1, \ldots+.
$$

It is assuned that for the lowest level in the group $T=-J$, for the noxt level $T=-J+1$, and so on.

The three principal mones of inertia of an asymetric top are $I_{A}, I_{B}$, and $I_{C}$ in wich $I_{A}<I_{B}<I_{C}$. The rotational constants in wich the vibrational infiusace is taken into account can be expressed as follows:-

$$
\begin{aligned}
& A_{v}=A_{e}-\sum \alpha_{i}^{(A)}\left(v_{i}+\frac{d_{i}}{b_{i}}\right) \\
& B_{v}=A_{e}-\sum \alpha_{i}^{(B)}\left(v_{i}+\frac{i}{2}\right) \\
& C_{v}=A_{e}-\sum \alpha_{i}^{(C)}\left(v_{i}+\frac{d}{2}\right)
\end{aligned}
$$


The respective $\alpha_{i}$ are small compared to the rotational constants for the equilibrium position.

$$
\begin{aligned}
& A_{e}=\frac{h}{8 \pi^{2} \mathrm{CI}_{\mathrm{A}}^{(e)}} \\
& \mathrm{B}_{e}=\frac{\mathrm{h}}{8 \pi^{2} \mathrm{C}_{\mathrm{B}}^{(\mathrm{e})}} \\
& \mathrm{C}_{\mathrm{e}}=\frac{\mathrm{h}}{8 \pi^{2} \mathrm{C}_{\mathrm{C}}^{(e)}}
\end{aligned}
$$

There is no direct method of getting the energy levels of the asymmetric top molecule. An approximate illustration of the energy levels of the asymmetric top molecule is given by connecting with smooth curves the two limiting cases of the symmetric top molecule (2). One in which $I_{B}=I_{C}$ (prolate) and the other in which $I_{B}=I_{A}$. (oblate). By letting $I_{B}$ decrease gradually from $I_{B}=I_{C}$ to $I_{B}=I_{A}$ we expect to find a continuous change of the energy levels.

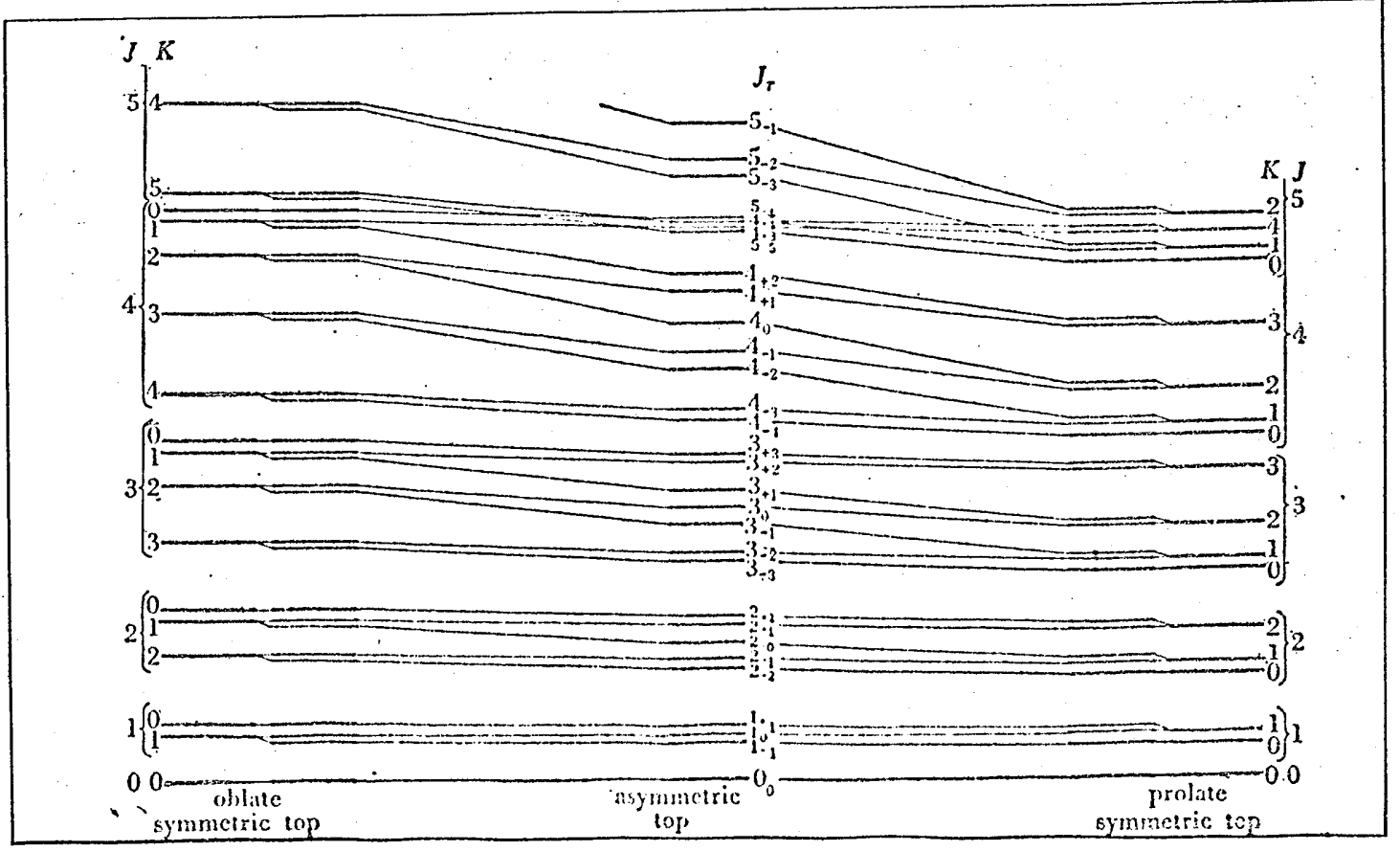

Fig. 7 - Energy levels of the asymmetric top; correlation to those of symmetric tops 
The diagram in Fig. 7 taken from Herzberg (2 page 45) shows the approximate energy levels of asymmetric top molecules obtained by joining by smooth curves the levels of a given $\mathrm{J}$ on the right, without intersection for low values of $\mathrm{J}$, with the levels of the same $J$ on the left.

In 1929 Wang (102) has shown that the splitting (K-type) increases with increasing $\mathrm{J}$ but much less rapidly for the higher $K$ values. Furthermore, the average of the levels of the same $\mathrm{K}$ deviates from a horizontal, that is, does not follow exactly $\frac{1}{2}(B+C) j(J \div 1)$. The average of all levels with a certain $\mathrm{J}$ follows accurately the formula

$$
\frac{\sum F\left(J_{T}\right)}{2 J+1}=\frac{1}{3}(A+B+C) J(J+1)
$$

This relation is very useful in checking the calculated levels and the correct assignment of the observed levels.

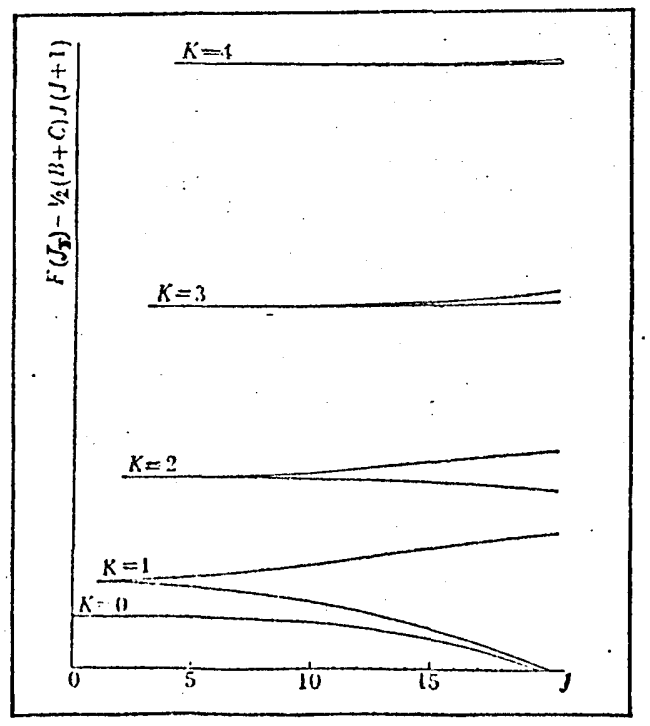

Fig. 8 - Rotational energy of a slightly asynmetric top (b about 0.01 ) as a function of $J$ (after Dieke and Kistiakowsky $(127))$ - The term $\frac{1}{2}(B+C) J(J+1)$ is subtracted from the energy, that is, the deviations of the curves from horizontal lines represent the deviations from the levels of the synmetric top. (2 page 49) 
10.2 Quantitative fomulas for the energy levels

The quantitative formule of the energy levels of the asymetric top molecule have been datemined by many authors. Arong them tho have been used in numbrical calculations. The first ono which is due to hang (102) is

$$
F\left(J_{T}\right)=1(B+O) J(J+1)+(A-1(B+C)) n_{T}
$$

The second one, due to kay (120) as corrected by King, fiatinor End Cross (121) is

$$
F\left(J_{T}\right)=\frac{1}{2}(A+C) J(J+1)+1(A-C) E_{T}
$$

The $(2 \mathrm{~J}+1)$ values of $\mathrm{H}_{\mathrm{T}}$ or $\mathrm{E}_{\mathrm{T}}$ for a given $\mathrm{J}$ are the roots of a secular deterainait of degree $(2 J+1)$. Fortunately this determinate can be factored into a nuber of deteminants of smaller degree leading to a numor of algobraic equations. The values of $n_{\mathrm{T}}$ or $\mathrm{E}_{\mathrm{T}}$ have been calculated for at least up to $\mathrm{J}=40$ (14).

For a given $\mathrm{J}$

$$
\dot{s}_{T}(k)=\ddot{B}_{-T}(-n)
$$

So Ray's equation is preferable because it is only needed to calculace the energy levels on one side of the anst asymotric 
case $(\mathrm{F}=0)$. Those on the other side are thea innediately given.

\subsection{Symetry properties of the total vave function}

In quantum mecinanics, according to the Eorn-Oppenheiner aproximation the total wave finction for a molecule can be expressed as a product of functions of various co-ordinates,

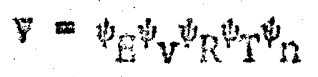

where the subscripts $E, V, R, T, n$ represent electronic, vibrational, rotational, translational, and nuelear spin co-ordinates respactively. Since the translational function $y_{T}$ does not involve the internal co-orinates it is therefore neglected. For most stable molecules the electronic ground state is totally symetric (7 p.129). Hence, in such a case, the symetry of the total wave function a bo obtained by finding the symetry of $\psi_{v}$, $\varphi_{R}$, and $\psi_{n}$ separately end tiren taking the direct product of their irreducible components. Symatry proporties of the vibracional weve function Since the vibrational cigenfunction is a function of the nomal co-orainates, its behaviour with respect to symetry operations deyonds upon the betaviour of tho nornal co-ordinates witi respect to tho symerry operations. Both the normal coordinates and the vibrationa wavefunctions have symotry properties corresponding to the various irreducible representations of the symatry point group for the rolecule. Thus, the 
symatry of the wavefunctions can bo explaind using group theory.

For cvery molecule the vibrational ground state is totally symetric end non-degenorate. The fundamontal wavefunction belongs to the same symetry species as the nomal comordinates. For noi-degenerate vibrations symetry sinecies of the n overtones nay be obtained fron the direct product of the irreducible representation of the corresponding fundanentel taken $(n+1)$ tines. That is, if a is odd the wavefuaction is totally symetric, if $\mathrm{n}$ is even its symetry spocies will be tire same as the fundamentel wavofunction. Symetry of the conbination and difference wavefunctions $\mathrm{cm}$ also be taken from the direct product of the irreducible representations of all the fundanental components in that level.

Symetery properties of the rotational wave function of asymatric top moleculics

For planar asymetric top moleculos there is no inversion coubling (2). The asymatric top rotor functions are distinguished by their bohaviour with respect to rotation by $180^{\circ}, \mathrm{c}_{2}^{\mathrm{a}}, \mathrm{c}_{2}^{\mathrm{b}}, \mathrm{c}_{2}^{\mathrm{c}}$, about the three principal axes (7).

For a rotation by $180^{\circ}$ about the axis of largest monent of inertia (c axis) in a totally syatetric vibrational anci electronic grourd stato the largest level $J_{+J}$ of each set of a given $J$ is tve, the tro next highest are $-v a$, the two next tro, and so on. For a rotation by $180^{\circ}$ about the axis of smallest moment of nertia (a axis) in a totally symetrical viorational and electronic Grouni state, the lowest icvel $J_{-J}$ of each set of a 
given $j$ is $+v e$, tite tho next higier are -ve, the next wo tve, and so on.

The syandry of the rotational energy levels with respect to a rotation by $180^{\circ}$ about the axis of internediate noment of inertia (b axis) in a totally symetrical vibrational and clectronic ground state can be easily found from the product of the tro signs (one belongs to the rotation about $c$ axis, the other boiongs to rotation about a nxis) for ench energy level. Thus, the symatry of the rotational energy levols of an asymatric top molecule nay be distinguished by their behaviour + or - with respect to the three operation, $\mathrm{C}_{2}^{a}, \mathrm{C}_{2}^{b}, \mathrm{C}_{2}$. Since one of these operations is equivalent to the other two carried out in succession, it is sufficient to ieteraine the behaviour with respect to tro of then; usually $\mathrm{C}_{2}{ }_{2}$ and $\mathrm{C}_{2}^{\mathrm{a}}$ are chosen. Therefore there are four different types of levels briefly described by,,+++-+ , and $-\rightarrow$ whore tho first sign rofers to the behaviour with respect to $c_{2}$, the second to the behaviour with respect to $\mathrm{C}_{2}^{\mathrm{a}}$.

Symatry properties of muclear spin wave function and statistical weight

In those molecules in which an exchange of idontical nuelei can be brought about by a rotation about one of the principal axes, tho total eigonfunction must be symotric (Bose-Einstoin statistics) or antisyzastric (Fomi-ilirac statistics) with respect to an exchange of the identical nuclei. 
In cases where there is only one pair of identical nuclei to exchange, the nubber of the synnetric nuclear spin wave functions is $(2 I+1)(I+1)$, and the number of the antisymetric nuclear spin wave functions is $(2 I+1) I$. Altogether there is a total of $(2 I+1)^{2}$ nuclear spin wave functions (18).

In cases of molecules in which there are more than one pair of identical nuciei that are exchanged by a rotation about a twofold axis, the resultant statistics must be considered. In general, the number of the symatric nuclear spin wave function can be derived fron the expression

$$
\frac{1}{2}\left[\left(2 I_{x}+1\right)^{2}\left(2 I_{y}+1\right)^{2}\left(2 I_{z}+1\right)^{2} \ldots+\left(2 I_{x}+1\right)\left(2 I_{y}+1\right)\left(2 I_{z}+1\right) \ldots\right]
$$

and the number of the entisymetric nuclear spin wave functions cen be derived from

$$
\left[\left(2 I_{x}+1\right)^{2}\left(2 I_{y}+1\right)^{2}\left(2 I_{z}+1\right)^{2} \cdots-\left(2 I_{x}+1\right)\left(2 I_{y}+1\right)\left(2 I_{z}+1\right) \ldots\right]
$$

10.8

where $I_{x}, I_{y}, I_{z} \ldots$ are the nuclear spin of each paix of identical nuclei that are exchanged by a rotation of $180^{\circ}$. The resultant statistics is Bose statistics if there is an even number of nuclei following Femi statistics in the group (XYZ . . ), it is Femi statistics if there is an odd number of nuciei following Fermi statistics (2). 
If all exchnged nuelei have zero spin, only those levels occur where the total eigenfunction is symetric vith respect to an excingnge of identical nuclei, that is, for a totally symatric electronic and vibrational state the antisymotric rotational; lovels aro missing.

For an asymatric top nolecule of point group $C_{2 h}$ with more tian one pair of ilentical nuclei that are exchanged by a rotation about the two-fold axis, the above symetry properties apply when $\mathrm{C}_{2}$ coincides with one of the three axes. Which levels have the greater statistical weight depends on the resultant statistics.

For gaseous oxalic acid there are four pairs of identical nuclei that are exchangea by a rotation of $180^{\circ}$ about the $z$ axis (symzetry axis). All oxchanged nucloi have spin zero except that of hydrogen with $\frac{1}{2}$ spin qumtum number. Thus, it is essumed that the rosultant statistics is Formi statistics. On applying 10.7 and 10.8 together with the Feni statistics the ratio of the statistical veight of the symetric and antisymetric rotational levels is $\frac{1}{3}$.

10.4 The energy level diagrat of the asymetric top molecule in the $\mathrm{C}_{2 \mathrm{~h}}$ point group

The rotational energy levels of the asymotric top malecule in the $\mathrm{C}_{2 \mathrm{~h}}$ point group may be distinguished by $\mathrm{A}$ (symetric) or B (antisymotric) with respect to a rotation by $180^{\circ}$, that is, by their behaviour + or - with respect to the same rotntion (2). 
If $\mathrm{C}_{2}$ coincides with the axis of the largest mont of inertia, the rotational levels t+ and t- are $A$, and tho levels at and -- are $\mathrm{B}$ rospectively.

If $C_{2}$ coincides with the axis of the internediate monent of incrtia, the rotational levels +\%, and - are A and the levels 4- and -4 are $B$ respectively.

If $\mathrm{C}_{2}$ coincicos with the axis of the smallest momont of inertia, the rotation levels to and -+ are $A$ and the levels +and - are B respectively. Hore the $+4,+-,+$ and - lovels heve the sme meaning as previously.

The rovibronic species may be obtained by

(1) the multipication rules, which for point group $C_{2 h}$ are

$$
\begin{aligned}
& A \times A=A \\
& A \times B=B \times A=B \\
& B \times B=A
\end{aligned}
$$

(2) adding tive subscript $g$ or $u$ into the rotational sub-group, depending on whether the vibronic state is $g$ or $u(7)$. Figs. 9 and 10 illustrate the energy levels of the symmetric top wolecule in the $\mathrm{C}_{2 \mathrm{~h}}$ point group (i.e. gaseous oxalic acid) when $\mathrm{C}_{2}$ coincides with the axis of Iargest moment of inertia. The firse sigas of the rotational levels $(+,+,-,+,-)$ wives for planar nolocules the 'parity', the behaviour witi raspect to the inversion, for vibrational states that are symetric with respect to the piane of the nolecule ( $\mathrm{Ag}$ and $\mathrm{Bg}$ ). For viorational states that are antisynutric with respect to this plane (Au, Bu) the parities are reversed. In these diegrans it is assumed that

(a) the electronic ground stato is totally symetric,

(b) the resultant statistics is Ferni statistics. 


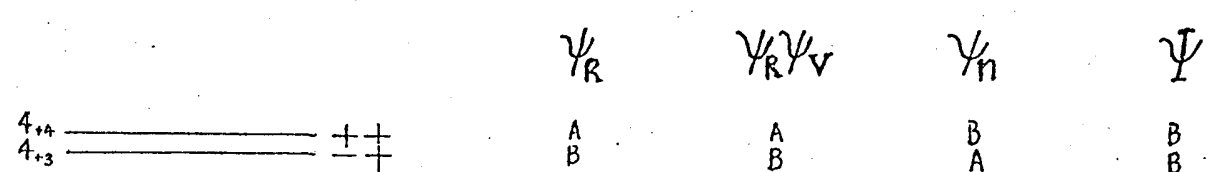

$\begin{array}{lllll}4+2= & B & B & A & B \\ 4+1= & B & A & B & B \\ 40 & & & & \\ 4-1= & A & A & B & B \\ 4-2= & & & & \\ = & B & B & A & B \\ B & B & B & A & B\end{array}$

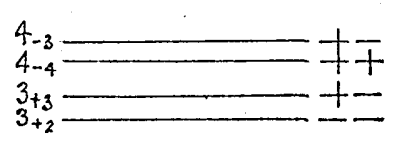

$\begin{array}{llll}A & A & B & B \\ A & A & B & B\end{array}$

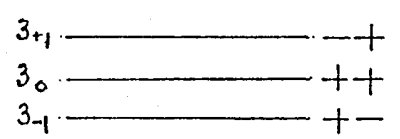

$\begin{array}{llll}B & B & A & B \\ A & A & B & B \\ A & A & B & B\end{array}$

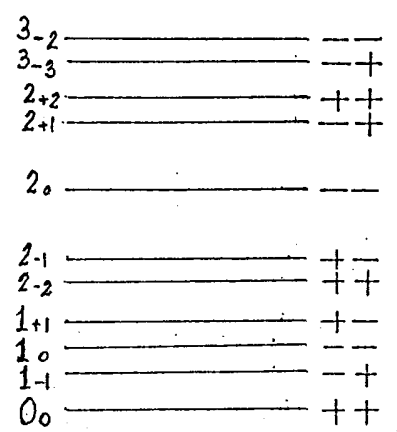

$\begin{array}{ll}B & B \\ B & B \\ A & A \\ B & B \\ B & B \\ A & A \\ A & A \\ A & A \\ B & B \\ B & B \\ A & A\end{array}$

$\begin{array}{ll}A & B \\ A & B \\ B & B \\ A & B \\ A & B \\ & B \\ B & B \\ B & B \\ B & B \\ A & B \\ A & B \\ B & B\end{array}$

\section{A vibrational level}

Fig. 9 - the rotational energy levels for $\mathrm{Ag}$ and $\mathrm{Au}$ vibrational levels of a $\mathrm{C}_{2} \mathrm{~h}$ molecule when $\mathrm{C}_{2}$ coincides with the axis of largest moment of inertia 

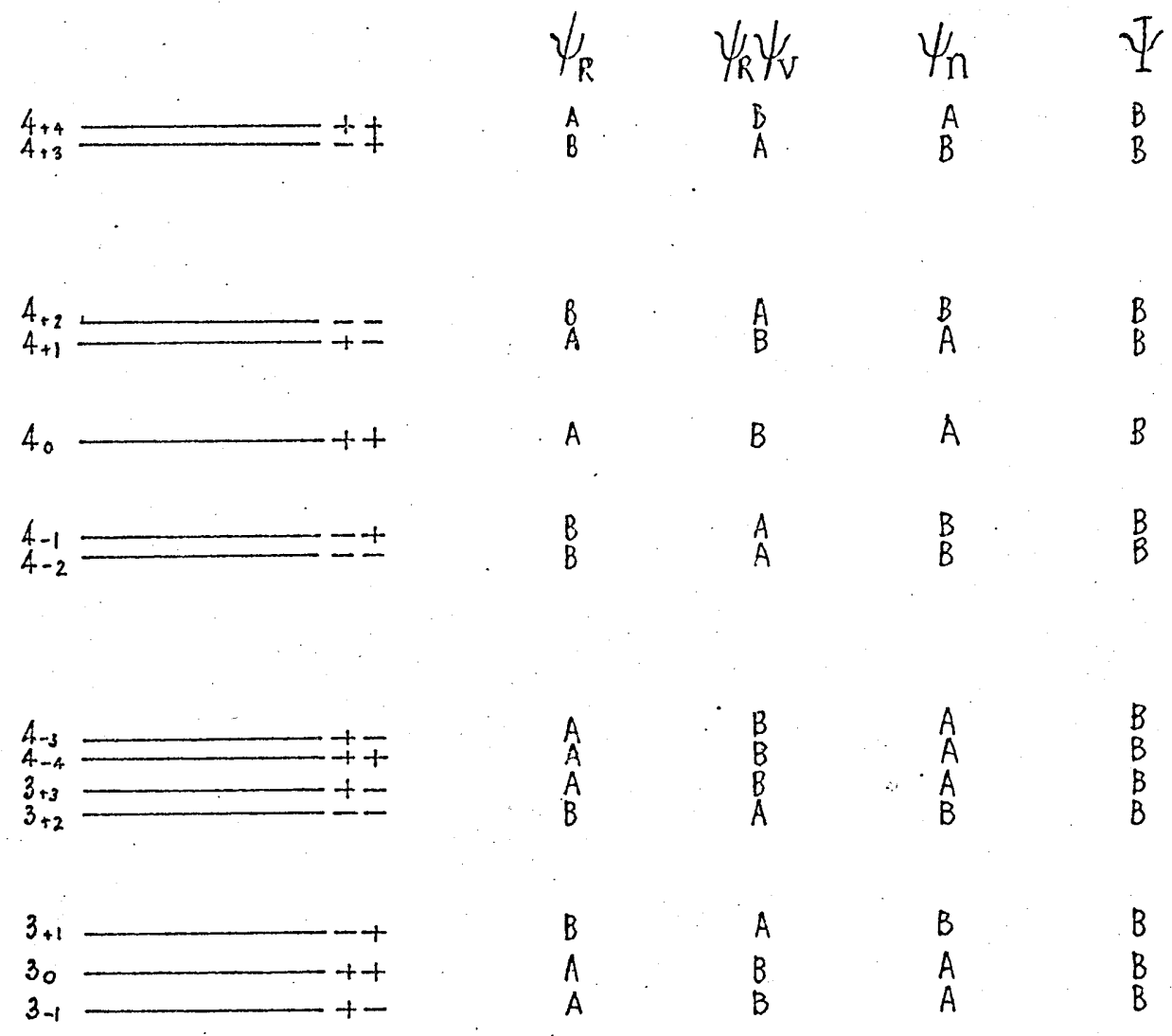

$\begin{array}{ll}B & A \\ A & B \\ A & B\end{array}$

$\begin{array}{ll}B & B \\ A & B \\ A & B\end{array}$
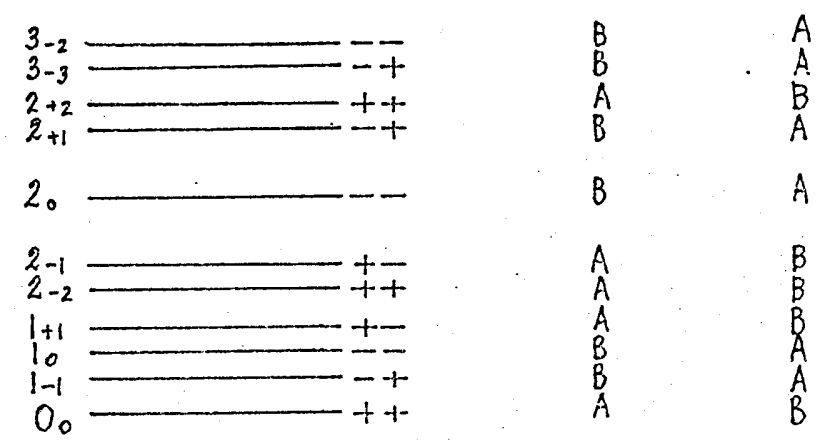

$\begin{array}{ll}B & B \\ B & B \\ A & B \\ B & B \\ B & B \\ A & B \\ A & B \\ A & B \\ A & B \\ B & B \\ A & B\end{array}$

B vibrational level

Fig. 10 - the rotational energy levels for the $\mathrm{Bg}$ and $\mathrm{Bu}$ vibrational levels of a $\mathrm{C}_{2} \mathrm{~h}$ molecule when $\mathrm{C}_{2}$ coincides with the axis of largest moment of inertia 


\section{CAPTLR 11}

\section{BOTATIOV-VIBRATION BELSCTION RULAS FOR}

ASTMETRTC TOR WLECULSS

11.1 Infrared rotation-vibration selection rules of asymetric top noleculos

The infrared rotation-vibration selection rules are assunad to be the sane as those for rotation and vibration soparateiy, except that it is now the direction of the change of dipole moment during the vibration that matters for the rotational selection rules (2). Thus, the selection rules are

$$
\begin{aligned}
& \Delta V= \pm 1, \pm 2, \ldots \ldots \ldots \mathrm{n} \\
& \Delta J=0, \pm 1 ; \quad J=0 \longleftrightarrow \mathrm{J}=0
\end{aligned}
$$

Furthenore, the selection rules for asynotric top molecules are different for the different orientations of the transition monent.

If the transition monent lies in the axis of least moment of inertia $(A-t y p e$ band), only the trausitions

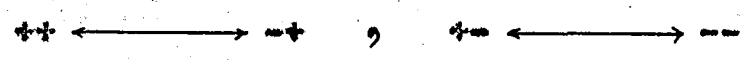

can take place.

If the transition monent lites in the exis of intenediate moant of inertia (B-type band), only the transitions

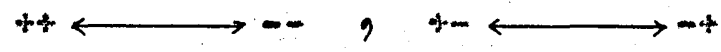


If the transition monent lies in the axis of largest monent of inertia ( $C$-type band), only the cransitions

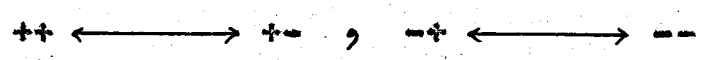

can taise place.

If the transition monents do not lie along any axis of moment of inertia, that is, it has more than one component along the principal axes, that transitionawill give rise to a hybrid badd. Thus, a hybrid band consists of a sunerposition of two or all three of $A-, B-$, and $C$ - types. The relative intensity contributions in a hybrid band depend upon the square of the dipole moment components along the inertial axes.

11.2 Theoretical infrared band envelopes of asymetric rotator molecules

For polyatonic rolecuies the resolution and the analysis of the rotational structure of the infrared bands appeax to be ratiner Iinited. The structure of the unresolved bands of esynnetric top nolecules has been stubied in great detail by many autiors.

The first paper was pullshed in 1938 by Badger and Zumfalt (26). They cerried out calculations conceming tine band envelopes of asyratetric top nolecules. Tiree types of bands, $A, B, C$, corresponding to the transitions in which the dipole romonts lie along the rinor, internaliate and major axes respectively, were calculated and drata for different sots of paraneter $p\left(=\frac{1}{3}, \frac{1}{2}\right.$, $\frac{3}{4}$ and $\left.\frac{5}{4}\right)$ and $S\left(=\frac{1}{2}, 0,+\frac{1}{2}\right)$ whore $\rho=\frac{A-C}{B}$ and $S=\frac{2 \pi-A-C}{A-C}$. 
The second paper was published by Varsanyi (112) in 1959 on the bend contours of the infrared vapour spectra of some dihalogenobenzene nolecules. Ho had reported an approximate procedure for deternining band contours and also applied it with success in analysing contours of bands in the infrared spectra of 1.3 diflurorbenzene, o-bromo-fluorobenzene and m-bronofluorobenzene.

The third paper was published by parkin (69) in 1965 . A computer program was developed to save the computer time and also allow the computer to accontodate a rucli larger scale problem. This progran calculated the frequencies and intensities of a band for a molecuie with any degree of asymetry in either of the conbining states, and integrated these ines into a contour which was printed out from the corputer in e direct usable form. The integrated envelope of the band was computed from assumed rolecular constants. It was then conpared with the observed contour, and then the constants were nodified until a sufficiently good fit was obtainod.

The fourth paper was pujlished by Seth paul and Dijkstra (55) on calculations of PR soparations for both symetric top and asymetric top molecules. A for useful expressions and tables of variable paranotors were given. The calculated PR separation of 87 molocules together with their observed values were also given in this paror.

Seth paul also pwishod 2 more papers in 1968. One of then published by himsolf (126) was a review paper on calculations 
of PR separations for all kinds of molecules. The other paper was pwilisted by hin and woyer (125) on the calculation of PK separations of hybrid bands in vapour phase infrared spectra. - The last paper was published in 1068 by Veda and Shinanouchi (71). The progran for the calculation of the band envelopes of asymatric top molecules that they used was similar to that of parkin. The $A, B$ and $C$ types envelopes were calculated for 40 typical molecules and were presented in this paper in grapinical fom. This is currentiy the most useful set of calculations available.

11.3 Raman rotation-vibration selection rules for the asymatric top inolecules

The Ranan rotation-vibration selection rules for asymetric top molecules have been derived by placzek and Teller (122). The vibrational selection rules are the sam as discussed before. The rotational selection rules are

$$
\Delta J=0, \pm 1, \pm 2\left(J^{\prime}+J^{\prime \prime} \geqslant 2\right)
$$

- and "indicate the initial and final states. All transitions between various rotational levels of the two vibrational states that obey these rules are possibie.

Furthenore, we have additional selection rules which depend on the components of the polarizability that are not zero for the particular vibrational transition. Let $x, y, z$ be the $a, b, c$ axes respectively. 


$$
\begin{aligned}
& \text { Generally, if the magnitude of at least one of }\left[\alpha_{x x}\right]^{n m} \text {, } \\
& {\left[\alpha_{y y}\right]^{\text {nn }} \text {, and }\left[\alpha_{z z}\right]^{n n} \text { are not zero, the transitions in which }} \\
& ++\longleftrightarrow++,+\longleftrightarrow+,-+\longleftrightarrow-+,-+\longleftrightarrow-
\end{aligned}
$$

are allowed.

If the magnitude of $\left[{ }^{\alpha_{x y}}\right]^{\mathrm{nn}}$ is not zero, the transitions in which

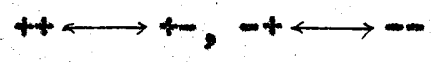

are allowed.

If the nagnitude of $\left[\alpha_{x z}\right]^{n n}$ is not zero, the transitions in which

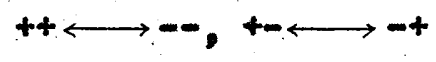

are allowed. which

If the magnitude of $\left[\alpha_{y z}\right]^{\mathrm{nn}}$ is not zero, the transitions in

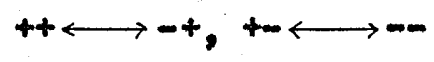

are allowed. 
For the totally symetric Raman bands the transitions between the rotational levels of the sane symmetry species of the two vibrational states are allowed. Since the rotational constants in the uppor and the lower states are very nearly the sane and the transitions in wich $\Delta J=0$ is possible, it is obvious that a large number of lines of the Q branch will coincide near the band origin. This sharp strong central maximm vill in general be the only feature that is observed.

For non-totally symetric Ranan bands the transitions between the rotational levels of different symetry species of the two vibrational states are allowed. Therefore, the lines of the $Q$ brancin do not, in general, coincide at the origin. Whether the bands form a central naxirum or not depends on the asymintry of the molecule and on the polarizability of the molecule. The three cases in which $\left[\alpha_{x y}\right]^{\mathrm{nm}} \neq 0,\left[\alpha_{x z}\right]^{\mathrm{nm}} \neq 0$, and $\left[\alpha_{y z}\right]^{\mathrm{nm}} \hat{\gamma}^{\prime} 0$ are comparable witin C-type, B-type, and A-type bands in infrared, except for the additional transitions $\Delta J= \pm 2$. In general the bands with $\left[a_{x z}\right]^{n m} \neq 0$ will have a central maximum, the other non-totally symetric Raman bands will not have such a sharp central maximu of outstanding intensity as to the totally symetric Raman bands, but will be more or less broad.

The observation of the width of Ramen lines gives a very significant way of observation of their state of polarization. In the case when a degree of dopolarization of $\frac{3}{4}$ is observed for a Raman line it is probabje, but not certain, that it corresponds to a non-totally symetric vibration, the observation 
of the line-width would confirn the assignnent. on the otirer hand, if the line is sharp, only the ones for which the degree of depolarization is less then will bo a totaliy symetric Raman lines (2). 


\section{CHAPTER 12}

VIBRATIONAL NDDES OF OXALIC ACID ARD REVIEI

OF PREVIOUS WORK

\subsection{Vibrational modes of gascous oxalic acid}

Gaseous oxalic acid is a trans-planar molecule. It contains a centre of symetry, a mirror plane $\left(\sigma_{h}\right)$, and a two-fold axis of rotation. Thus, it is classified in $\mathrm{C}_{2 \mathrm{~h}}$ point group.

Table 3

\begin{tabular}{|c|r|r|r|r|l|l|}
\hline $\mathrm{C}_{2 \mathrm{~h}}$ & $\mathrm{E}$ & $\mathrm{C}_{2}$ & $\mathrm{i}$ & $\sigma_{\mathrm{h}}$ & & \\
\hline $\mathrm{Ag}$ & 1 & 1 & 1 & 1 & $\mathrm{R}_{\mathrm{z}}$ & $\mathrm{x}^{2}, \mathrm{y}^{2}, \mathrm{z}^{2}, \mathrm{xy}$ \\
$\mathrm{Bg}$ & 1 & -1 & 1 & -1 & $\mathrm{R}_{\mathrm{x}}, \mathrm{R}_{\mathrm{y}}$ & $\mathrm{xz}, \mathrm{yz}$ \\
$\mathrm{Au}$ & 1 & 1 & -1 & -1 & $\mathrm{~T}_{z}$ & \\
$\mathrm{Bu}$ & 1 & -1 & -1 & 1 & $\mathrm{~T}_{x}, \mathrm{~T}_{y}$ & \\
\hline
\end{tabular}

Being an eight atomic molecule, the gaseous oxalic acid has 18 findanental modes of vibration.

Table 4

\begin{tabular}{|l|c|c|c|c|}
\hline$c_{2 h}$ & $\mathrm{I}$ & $c_{2}(z)$ & $\mathrm{i}$ & $\sigma(x y)$ \\
\hline$r_{\text {total }}$ & 24 & 0 & 0 & 8 \\
$r_{\text {trans. }}$ & 3 & -1 & -3 & 1 \\
$r_{\text {rot }}$ & 3 & -1 & 3 & -1 \\
$r_{\text {vib. }}$ & 18 & 2 & 0 & 8 \\
\hline$r_{\text {vib. }}-7 \mathrm{Ag}+2 \mathrm{Bg}+3 \mathrm{Au}+6 \mathrm{Bu}$ \\
\hline
\end{tabular}


Table 4 shows the symatry species of the modes of vibration of gaseous oxalic acid as obtained by group theory. A11 atons are in the $x y$ plane and $z$ is the two-fold axis.

Applying the rule of mutual exclusion it is not expected to see Ranan fundanentals in the infrared spectrum, and vice versa. The types of vibration and activity are show in Table 5.

Table 5

\begin{tabular}{|c|c|l|l|}
\hline Species & Nunber & \multicolumn{1}{|c|}{ Type } & Activity \\
\hline $\mathrm{Ag}$ & 7 & in plane & Raman \\
$\mathrm{Bg}$ & 2 & out of plane & Raman \\
$\mathrm{Au}$ & 3 & out of plane & Infrared \\
$\mathrm{Bu}$ & 0 & in plane & Infrared \\
\hline
\end{tabular}

12.2 Review of previous work on gaseous oxalic ecid

One paper on gaseous oxalic acid was published before this work started by Pava and Stafford (93). The infrared spectrum was recorded between $4000 \mathrm{ca}^{-1}$ to $400 \mathrm{~cm}^{-1}$. Only 4 gaseous oxalic acid bands were observed at $3485 \mathrm{~cm}^{-1}, 1820 \mathrm{~cm}^{-1}, 1325 \mathrm{~cm}^{-1}$, and $1240 \mathrm{~cm}^{-1}$ at temperatures between $110^{\circ}-140^{\circ} \mathrm{C}$. tho gaseous oxalic acid bands were observed between $1000 \mathrm{~cm}^{-1}$ to $400 \mathrm{~cm}^{-1}$. All observed frequencios agree woll witi the present work except the band at $1240 \mathrm{~cm}^{-1}$ which was observed at $1275 \mathrm{~cm}^{-1}$ in tmy work. A spectrun of deuterated oxalic acid was also recorded at approximately the same teanorature and was presented in their paper. 
Three bands of deuterated oxalic acid were observed at $2575 \mathrm{~cm}^{-1}$, $1810 \mathrm{~cm}^{-1}$, and $1220 \mathrm{~cm}^{-1}$ respectively. Assignment of two bands, $0-\mathrm{H}$ stretch and $\mathrm{C}=\mathrm{O}$ stretch, were made without any calculation. It was reported that spectra of gaseous oxalic acid and deuterated oxalic acid were first observed at approximately $105^{\circ} \mathrm{C}$.

According to their work the spectrum of gaseous oxalic acid recorded was subject to interference not oniy by solid oxalic acid fram the condensation process, but also by the high background caused by decomposition products.

\section{Table 6}

Inirared absorption frequencies of gaseous oxalic acid

and deuterated oxalic ecid observed by

Pava and Stafford

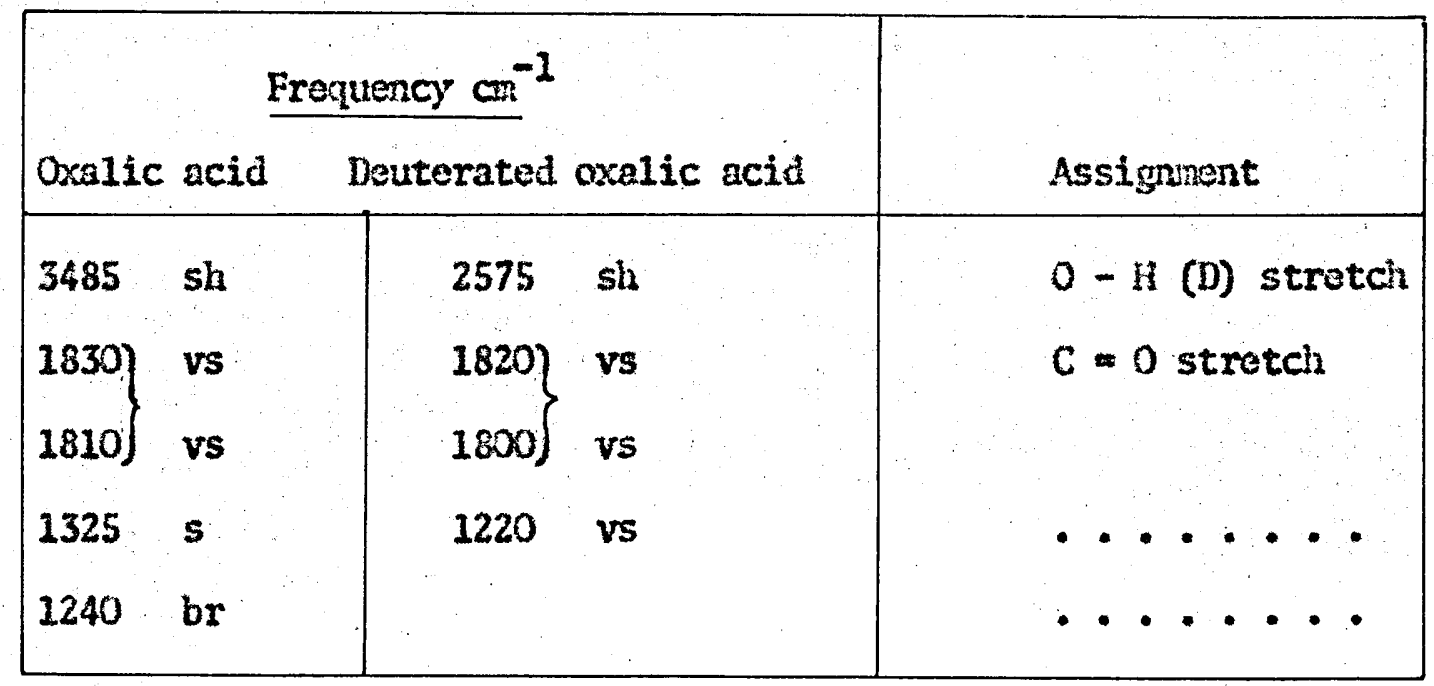

sh $=$ sharp, vs = vory strong, s = strong, br = broad 
A second paper appeared during the course of this work. This was published by Bardet, Meury and Tabacik (109). Infrared bands of gaseous oxalic acid were observed at approxinately $112^{\circ} \mathrm{C}$. Raman spectra of oxalic acid in aqueous solution and in the crystal phase ( $a$-form) were also roported in the same paper. Assignments of both infrared and Raman bands were made.

Unfortmately, although they observed more gaseous oxalic acid infrared absorption frequencies than proviously, this paper was badly written for the following reasons:(1) No spectra were presented in this paper. (2) Although the authors natitioned that the calculations were made using the structure given by Shibata and Kinura (104) and the Urey-Bradley force field, they did not give any details of their calculations at all.

(3) If they had made a calculation at least

(a) a set of force constants should be given;

(b) a set of calculated frequencies should be given. (4) No relative intensitios for either infrared or Ranan bands were given.

(5) Some assignments are very doubtful. Firstiy, they did not nention the presence of impurity bands. Tho bands at $1760 \mathrm{~cm}^{-1}$ and $1105 \mathrm{~cm}^{-1}$, which they assigned to gaseous oxalic acid, wore found in my work to be formic acid bands. Secondly, they assigned difforence bands by ignoring the corresponding conbination bands. Two bands at $1364 \mathrm{~cm}^{-1}$ and $720 \mathrm{~cm}^{-1}$ wore assigned to difference bands without the corresponding bands at $2119 \mathrm{cta}^{-1}$ and 
$1825 \mathrm{~cm}^{-1}$ being observed. According to theory (2) it is expected that a conbination band is stronger than the difforence band by the Boltzman factor

$$
\frac{I\left(v_{j}-v_{i}\right)}{I\left(v_{j}+v_{i}\right)}<e^{-h c v_{i}} \frac{k T}{k T}
$$

where $v$ is expressed in wave numbers.

\section{Table 7}

Infrared absorption frequencies of gaseous oxalic acid observed by Bardet, Floury, and Tabacik

\begin{tabular}{|c|c|c|}
\hline $\begin{array}{l}\text { band position } \\
(\mathrm{cm})\end{array}$ & $\begin{array}{l}\text { their assigment } \\
\text { mode species }\end{array}$ & Compare with the present work \\
\hline 3484 & $\mathrm{Bu}$ & agree \\
\hline 1820 & $v_{9}$ & agree \\
\hline 1760 & $v_{10^{+} v_{7}}$ & disagree (formic acid) \\
\hline 1364 & $v_{2}-v_{13}$ & disagree (no combination band) \\
\hline 2324 & $v_{10}$ & agree \\
\hline 1280 & $v_{11}$ & agree \\
\hline 1105 & $v_{7}+v_{16}$ & disagree (formic acid) \\
\hline 949 & $v_{13}+v_{15}$ & not observed \\
\hline 913 & $v_{7}+v_{17}$ & not observed \\
\hline 821 & $v_{7}+v_{13}$ & disegree (impurity) \\
\hline 720 & $v_{11}-v_{15}$ & disagree (no combination band) \\
\hline 668 & $v_{16}$ & agreo \\
\hline
\end{tabular}




\begin{tabular}{|c|lc|l|}
\hline $\begin{array}{c}\text { band position } \\
\left(\mathrm{cm}^{-1}\right)\end{array}$ & $\begin{array}{l}\text { their assignnent } \\
\text { mode }\end{array}$ & species & Compare with the present work \\
\hline 565 & $v_{12}$ & $\mathrm{Bu}$ & not observed \\
452 & $v_{17}$ & $\mathrm{Au}$ & agres \\
380 & $v_{13}$ & $\mathrm{Bu}$ & not observed \\
\hline
\end{tabular}

Thiey have given different symbols for the inplane vibrational ardes. Thus, for simplicity, Table 8 is given.

\section{Table 8}

Inplane fundanental nodes of gaseous oxalic acid

ny symbois their synbols planar modes of vibration

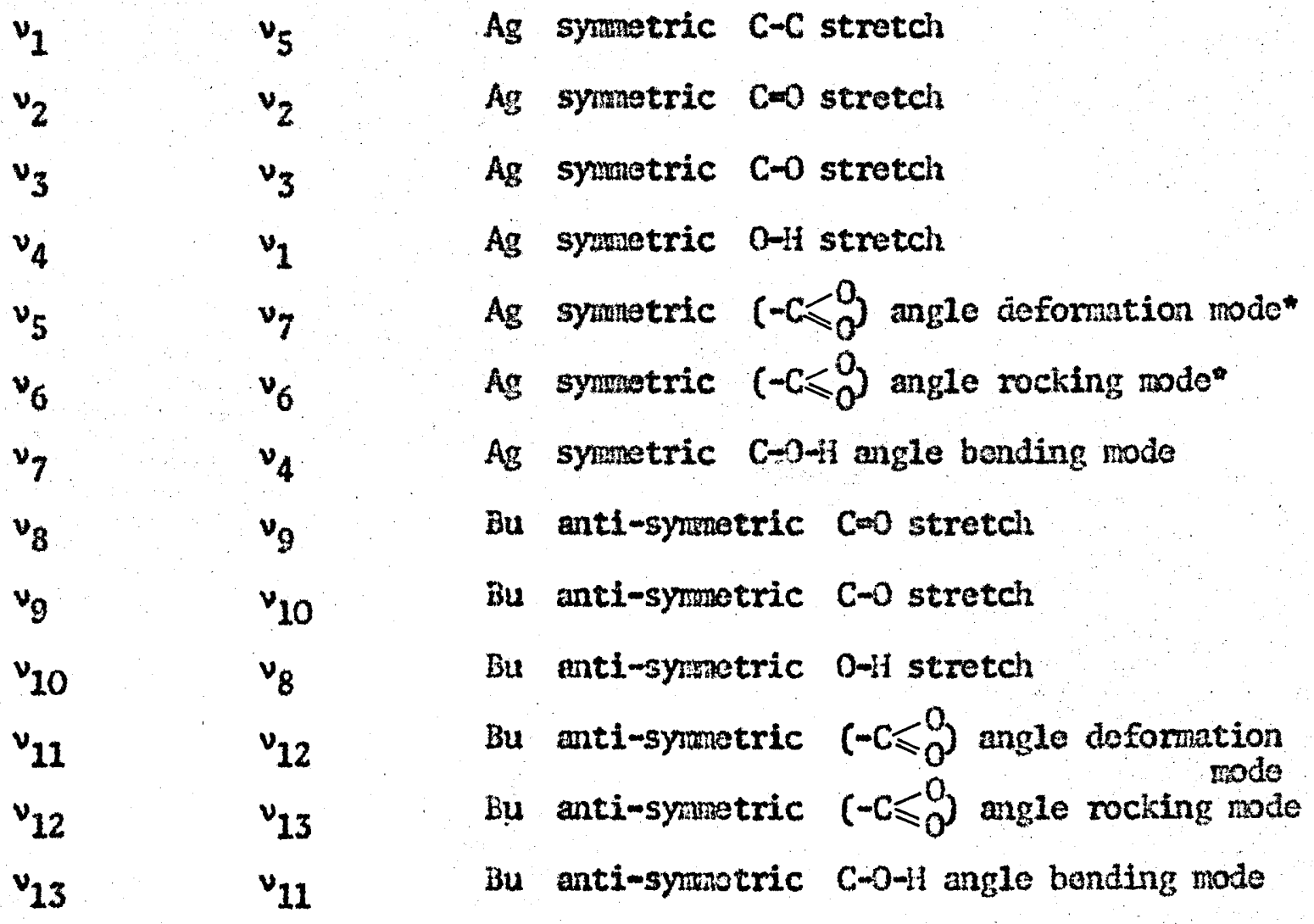


A third paper on gascous oxalic acid also appeared during the course of this work. This paper by Cyvin and Alfneim (79) deals with molecular vibrations and mean amplitudes of oxalic acid nonower. They did not perfom any experiment. The calculation was made using one oxalic acid band fron Nahlovska, Nahlovsky and Serand (78), the observed data from Marata and Kavai (51) wisich was the Ranan spectrum of oxalic acid solution in mothanol, and the observed infrared dota of Pava and Stafford (98) nentioned above. The out of plane frequencies were calculated from force constants from fomic acid and acetic acid nonomers.

\section{Table 9}

Inplane calculated frequencies for oxalic acid nonomer

from Cyvin and Alfheim (79)

\begin{tabular}{|c|c|c|l|l|}
\hline Species & Wo. & $v\left(\mathrm{~cm}^{-1}\right)$ & origin & approximate mode \\
\hline $\mathrm{Ag}$ & 1 & 3505 & calc. & $v(\mathrm{O}-\mathrm{i})$ \\
$\mathrm{Ag}$ & 2 & 2237 & caic. & $v(\mathrm{C}=0)$ \\
$\mathrm{Ag}$ & 3 & 1762 & soln. (51) & $v(\mathrm{C}-\mathrm{C})$ \\
$\mathrm{Ag}$ & 4 & 1457 & soln. (51) & $\delta(\mathrm{C}-0-\mathrm{H})$ \\
$\mathrm{Ag}$ & 5 & 852 & soln. (51) & $\delta(\mathrm{C}-\mathrm{C}=0)$ \\
$\mathrm{Ag}$ & 6 & 590 & soln. (51) & $v(\mathrm{C}-0)$ \\
$\mathrm{Ag}$ & 7 & 432 & soln. (51) & $\delta(\mathrm{C}-\mathrm{C}-0)$ \\
$\mathrm{Bu}$ & 8 & 3475 & vapour(78) & $v(\mathrm{O}-\mathrm{H})$ \\
$\mathrm{Bu}$ & 9 & 1820 & vapour(98) & $v(\mathrm{C}=0)$ \\
$\mathrm{Bu}$ & 10 & 1325 & vapour(98) & $\delta(\mathrm{C}-\mathrm{O}-\mathrm{H})$ \\
\hline
\end{tabular}




\begin{tabular}{|c|c|c|l|l|}
\hline Species & No. & $v\left(\mathrm{ca}^{-1}\right)$ & origin & approxinate node \\
\hline $\mathrm{Bu}$ & 11 & 1240 & vapour $(98)$ & $v(\mathrm{C}-0)$ \\
$\mathrm{Bu}$ & 12 & 656 & $\mathrm{calc}$. & $\delta(\mathrm{C}-\mathrm{C}=0)$ \\
$\mathrm{Bu}$ & 15 & 480 & calc. & $\delta(\mathrm{C}-\mathrm{C}-0)$ \\
\hline $\mathrm{Bg}$ & 1 & 869 & calc. & - \\
$\mathrm{Bg}$ & 2 & 505 & calc. & - \\
$\mathrm{Au}$ & 1 & 696 & calc. & - \\
$\mathrm{Au}$ & 2 & 471 & calc. & - \\
$\mathrm{Au}$ & 3 & 90 & calc. & - \\
\hline
\end{tabular}

Although most of the calculated infrared frequencies (Bu) are fitted well with the observed frequencies, it was found that:-

(1) Their calculated Ramen frequencies do not agree with the observed Raman frequencies of gaseous oxalic acid in my present work, e.g. the observed Raman band at $1185 \mathrm{~cm}^{-1}$.

(2) Their calculated Ranan Erequencies and their assignients are completely different from my work.

(3) The calculated Raman frequency $\left(2237 \mathrm{~cm}^{-1}\right)$ of the symetric Coo stretch is ruch higher than one would expect for acid carbonyl vibrations.

12.3 Surnary of provious rork on solid oxalic acid and solutions

Solid oxalic acid exists in three foras $a, \beta$, and dihydrate as shown in Figures 11, 12,13 respectively. The structure of eacin form has boen published in many papers using 
both $X$-rays and neutron diffraction methods (86), (87), (88), (89), (90), (91), (92), (93).

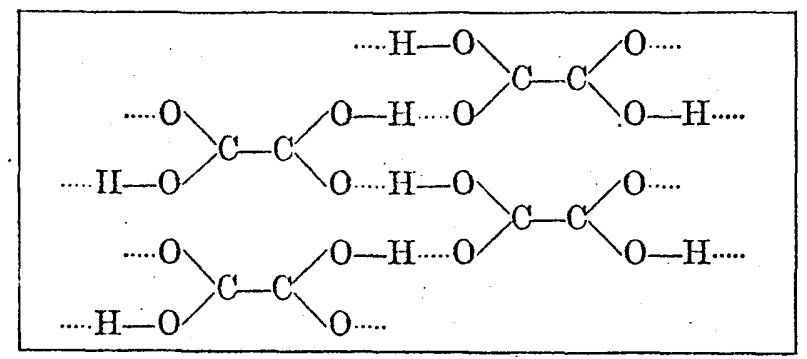

Fig. 11

$\alpha$-oxalic acid

In $\alpha$-oxalic acid the carbonyl and hydroxyl groups of a single $\mathrm{COOH}$ are each associated with different oxalic acid molecules giving rise to a planar sheet structure.

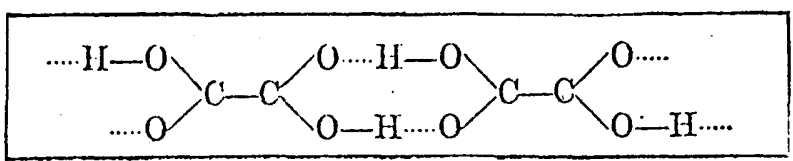

Fig. 12

$\underline{\text { B-oxalic acid }}$

In $\beta$-oxalic acid the molecules are linked together in long chains by cyclic hydrogen bonds. 


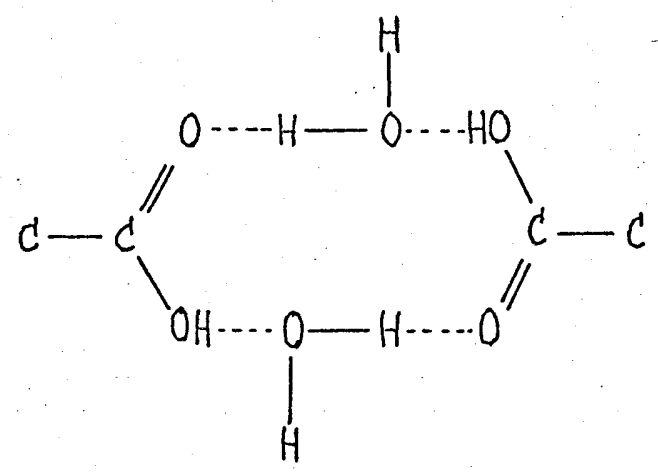

\section{Fig. 13}

\section{Oxalic acid dihydrate}

The structure of the dihydrate is similar to that of the $\beta$-form but with two molecules of water symmetrically placed between the carbonyl groups.

So far no complete work on the vibrational spectrum of solid oxalic acid has been made. The following papers have been published on the spectra of oxalic acid in solution and in the solid phase.

(1) Marigan and Bardet (124) published a paper on the Raman spectrum of oxalic acid dihydrate crystal. In this paper 7 bands were observed, one band at $1647 \mathrm{~cm}^{-1}$ was assigned to the vibrational mode of $\mathrm{H}_{2} \mathrm{O}$, the other six were assigned to oxalic acid dihydrate bands at $449 \mathrm{~cm}^{-1}, 575 \mathrm{~cm}^{-1}, 724 \mathrm{~cm}^{-1}, 850 \mathrm{~cm}^{-1}$, $1469 \mathrm{~cm}^{-1}$, and $1761 \mathrm{~cm}^{-1}$.

(2) Murata and Kawai (51) published their work on the infrared and Raman spectra of oxalic acid solution in methanol. Four 
strong bands at $432 \mathrm{~cm}^{-1}, 852 \mathrm{~cm}^{-1}, 1457 \mathrm{~cm}^{-1}$, and $1762 \mathrm{~cm}^{-1}$. and one weak band at $590 \mathrm{chin}^{-1}$ were observed in the Ranan spectrum. In the infrared only three bands at $720 \mathrm{~cm}^{-1}, 1243 \mathrm{~cm}^{-1}$, and $1650 \mathrm{~cm}^{-1}$ were observed.

(3) Bellamy and Pace (62) published a long paper on the infrared spectrun of solid oxalic acid. In this paper infrared spectra of $\alpha, \beta$, and the dihydrate forms of oxalic acid including a table of bands and some assigments were given.

(4) Lorenzelli and Alcnagna (110) published a paper on the far infrared spectrum of oxalic acid dihyrate betreen $400 \mathrm{~cm}^{-1}$ $70 \mathrm{~cm}^{-1}$.

(5) The work of Bardet, Fleury and Tabacik (109) has been considered in section 12.2. Thoy included some data on solution and solid phase spectra as well as the vapour phase results already discussed.

Fro: the papers above it is obvious that the difforences in the solid oxalic acid structures result in differences in their vibrational spectra. Since the structure of gaseous oxalic acid is difforont from that of the solid and also this work is dealing with the infrared and Raman spectra of gaseous oxalic acid, we will not discuss the vibrational spectre of solid oxelic acid anymore. 


\section{QIAPTER 13}

PIYSICAL AND CEEMTAL PRORERTIES OF OXALIC ACID

13.1 physical and chomical proportios

The physical and chomical properties of oxalic acid were published in (73). The sublimation of the anhydrous oxalic acid starts at a temperature sonewhat below $100^{\circ} \mathrm{C}$ and proceeds rapidiy at $125^{\circ} \mathrm{C}$. Partial decomposition takes place before the melting point at $185-190^{\circ} \mathrm{C}$, and its decomposition products include fomic acid, carbon nonoxide, carion dioxide, and water. Dahydrating agents, such as sulphuric acid, accelerate the themal decomposition of all forms of the acid. The anhydrous acid is quito soluble in anhydrous ethyl ether, $23.6 \mathrm{gm}$ por $100 \mathrm{gm}$ of solvent, while the corresponding value for the dihydrate is $1.47 \mathrm{gm}$ per 100 gm. Anlydrous oxalic acid nay be crystallised from wara nittric or sulphuric acid.

Oxalic acid crystallises from water as the dihydrate but readily loses its water of crystallisation wen heated rapidly. Complete removal of water may be accomplished by heating under reduced pressuro or by refluxing in a low boiling solvent capable of foming a water azcotrope. Its solubility in water increases with temperature. Approxinate solubility values are given by the following formulas: from $0^{\circ} \mathrm{C}$ to $60^{\circ} \mathrm{C}$ the solubility is given by

$$
S=3.42+0.168 t+0.0048 t^{2}
$$


and from $50^{\circ} \mathrm{C}$ to $90^{\circ} \mathrm{C}$ by

$$
S=0.333 t+0.003 t^{2}
$$

where $S=g(C o d)_{2}$ per $100 \mathrm{gm}$ solution, $t={ }^{\circ} \mathrm{C}$.

\section{Table 10}

Pinsical and chemical properties of oxalic acid

Oxalic acid anhydrous, $(\mathrm{COOH})_{2}$ (presumably a form)

lolting point

vapour pressure, range $60^{\circ} \mathrm{C}-105^{\circ} \mathrm{C}$

jiecific heat, range $-200-+50^{\circ} \mathrm{C}$

hoat of conbustion

heat of formation at $18^{\circ} \mathrm{C}$

heat of solution (in water)

heat of sublination

heat of decomposition

themal conductivity at $0^{\circ} \mathrm{C}$

ionization constant, $k_{1}$

$x_{2}$

coefficient of expansion at $25^{\circ} \mathrm{C}$ $187^{\circ} \mathrm{C}$

$\log _{\mathrm{ym}}=-(4726.95 / \mathrm{T})+12.2229$

$c_{\mathrm{p}}=0.259+0.00076 \mathrm{~T}$

$60.1 \mathrm{kcal} / \mathrm{mole}$

$105.36 \mathrm{kcal} / \mathrm{rrolo}$

$-9.58 \mathrm{~kJ} / \mathrm{mole}$

$21.65 \mathrm{kcal} / \mathrm{mole}$

$197.60 \mathrm{keai} / \mathrm{kolo}$

$9.0 \times 10^{-3}$ watt $/\left(\mathrm{cm}^{2}\right)\left({ }^{\circ} \mathrm{C} / \mathrm{cm}\right)$

$6.5 \times 10^{-2}$

$6.0 \times 10^{-5}$

$1.784 \times 10^{-4} \mathrm{ml} /(\mathrm{gm})\left({ }^{\circ} \mathrm{C}\right)$

Oxalic acid dihydrate $(000 \mathrm{H})_{2} \cdot 2 \mathrm{H}_{2} \mathrm{O}$

melting point

density, $\mathrm{d}^{20}$

refractive index, $\mathrm{n}^{20} \mathrm{D}$

heat of solution (in water)
101.5

1.653

1.475

$-35.5 \mathrm{~kJ} / \mathrm{mole}$ 
13.2 Vapour pressure of anhydrous oxalic acid

In $1926 \%$. Albore Woyes Ir., and Delbert E. Wobbo worked out an approximate equation to calculate the vapour pressure of anhydrous oxalic acid at different temperatures.

Since at tarperatures higher than $105^{\circ} \mathrm{C}$ the oxalic acid decomposes quite rapidly it is obvious that

(a) direct measurenents of the vapour pressure are very difficult to carry out;

(b) the values obtained would be of doubtful accuracy. Hence they performed the vapour pressure weasurements at temperatures ranging from $60^{\circ} \mathrm{C}$ to $105^{\circ} \mathrm{C}$. The deserved values agreo well with the calculated values using the expression

$$
\log _{10} p=-(4726.95 / T) \div 12.223
$$

$P$ is expressed in millinetres and temperature is expressed in degrees absolute.

It is assumed that this expression can be used for the purpose of extrapolation. A graph which shows the relation between the vapour pressure and the temperature is then obtained. 


\section{Table 11}

The calculated pressure of aninydrous oxalic acid at different tomperatures

\begin{tabular}{|c|c|}
\hline Temperature ${ }^{\circ} \mathrm{C}$ & Calculated pressure in ma \\
\hline 80 & 0.068 \\
90 & 0.159 \\
100 & 0.355 \\
110 & 0.759 \\
120 & 1.567 \\
130 & 3.116 \\
\hline
\end{tabular}




\subsection{Mblecular structure of gascous oxalic acid}

Two papers ware published on the structure of gaseous oxalic acid using the electron diffraction method.

The first paper was published before this work started by Shibata and xinura (104). They interpretei their results on the basis of a planar trans-confomation (Fig. 15) with the following paransters:-

$$
\begin{aligned}
C-C \text { distance } & =1.54^{\circ} \mathrm{A}=0.00^{\circ} \mathrm{A} \\
\mathrm{C}=0 \text { distance } & =1.22^{\circ} \mathrm{A} \pm 0.02^{\circ} \mathrm{A} \\
\mathrm{C}-O \text { distance } & =1.37^{\circ} \mathrm{A}=0.02^{\circ} \mathrm{A} \\
\angle O=C-O & =125^{\circ} \pm 3^{\circ} \\
\angle O=C-C & =122^{\circ} \pm 5^{\circ}
\end{aligned}
$$

In thear calculation they assumed that the scattering effect of hydrogen atons is negligible.

The second paper appeared during the course of this work and was publishod by Nahlovska, Nahlovsky and Strand(78). They also interpreced their results on the basis of planar trans-conformation with the following parameters:-

$$
\begin{aligned}
C-C \text { distance } & =1.548 \mathrm{~A} \pm 0.004 \mathrm{~A} \\
\mathrm{C}=\mathrm{O} \text { distance } & =1.203 \mathrm{~A} \pm 0.001 \mathrm{~A} \\
\mathrm{C}-\mathrm{O} \text { distance } & =1.339 \mathrm{~A} \pm 0.002 \mathrm{~A} \\
O-\mathrm{H} \text { distance } & =1.056 \mathrm{~A} \pm 0.014 \mathrm{~A} \\
\angle O=\mathrm{C}-\mathrm{O} & =125^{\circ} \pm 0.2^{\circ} \\
\angle O=\mathrm{C}-\mathrm{C} & =123.1^{\circ} \pm 0.9^{\circ} \\
\angle \mathrm{C}-\mathrm{O}-\mathrm{H} & =104.4^{\circ} \pm 2.3^{\circ}
\end{aligned}
$$


Two models of gaseous oxalic acid were suggested in the latter paper. In the first model the $\mathrm{O}-\mathrm{H}$ bonds pointed away from the neighbouring carbonyl oxygens as shown in Fig. 15. The second model was obtained by rotating the hydrogen atoms $180^{\circ}$ around the $\mathrm{C}-0$ bonds as shown in Fig. 16. With the $0-\mathrm{H}$ bonds pointing towards the neighbouring carbonyl oxygens, hydrogen bonds are formed. Both models fitted the electron diffraction data quite well.

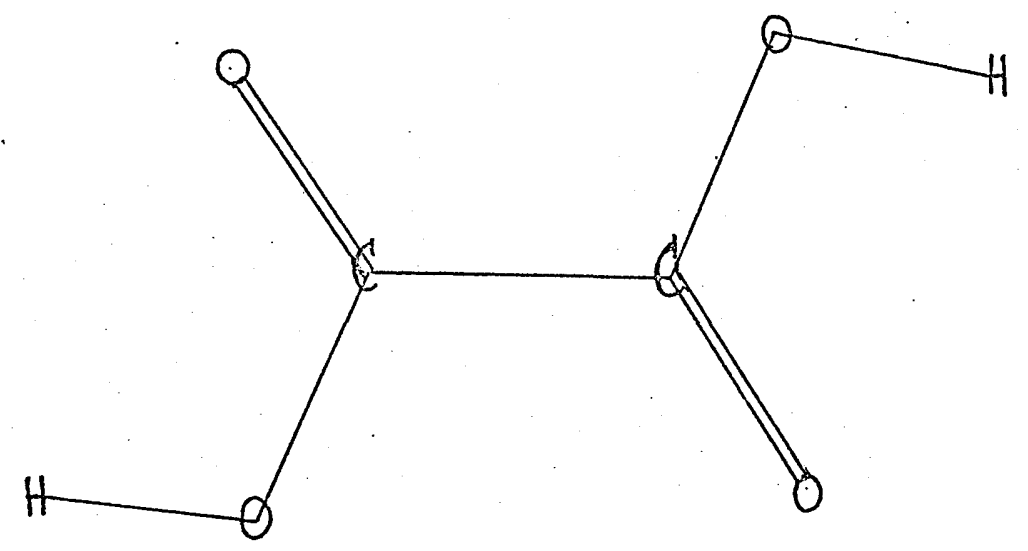

Fig. 15

First mode1

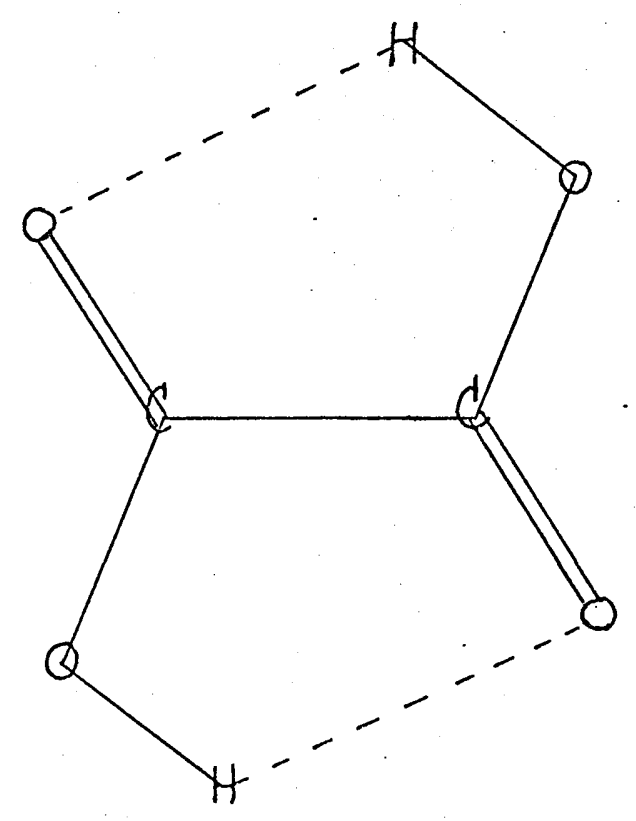

Fig. 16

Second mode1 
The authors suggested that the structure of gaseous oxalic acid is more likely to be the second model bocause of

(a) the frequoncy shift of the 0 - I stretching band from the free 0 - II stretching band position;

(b) the 0 - If bond length is longer than the free 0 - I bond, which they thought ves caused by the weak intramolecular effect of $\mathrm{H}$ bond.

\section{Comment}

I would like to say that the evidence that they have given is not yet enough to conclude that there are $1 \mathrm{H}$-bonds in the single molecule because of the following reasons:-

(a) Usually when there is H-bonding the 0 - H asymetric stretchings frequency is accompanied by band broadoning. According to the spectrum recorded this band is at $3475 \mathrm{~cm}^{-1}$, approximately $280 \mathrm{~cm}^{-1}$ from the $0-11$ asymetric stretch of water (2), and it is quito sharp.

(b) At room temperature the oxalic acid becones polynerized. It is nore likely that with the first model it will be easier for free miecules to become polymers.

(c) As both models fitced the electron diffraction data quite well, thus, more infonation is required before the conclusion is made.

With the ahovo possibilities in mind, the subsequent calculations are made on both models. 


\section{CHAPTER 14}

\section{PRINCIPAL MOMENTS OF INERTIA AND PRINCIPAL AXES}

\section{OF GASEOUS OXALIC ACID}

14.1 (a) Principal moments of inertia of the gaseous oxalic acid

First mode1
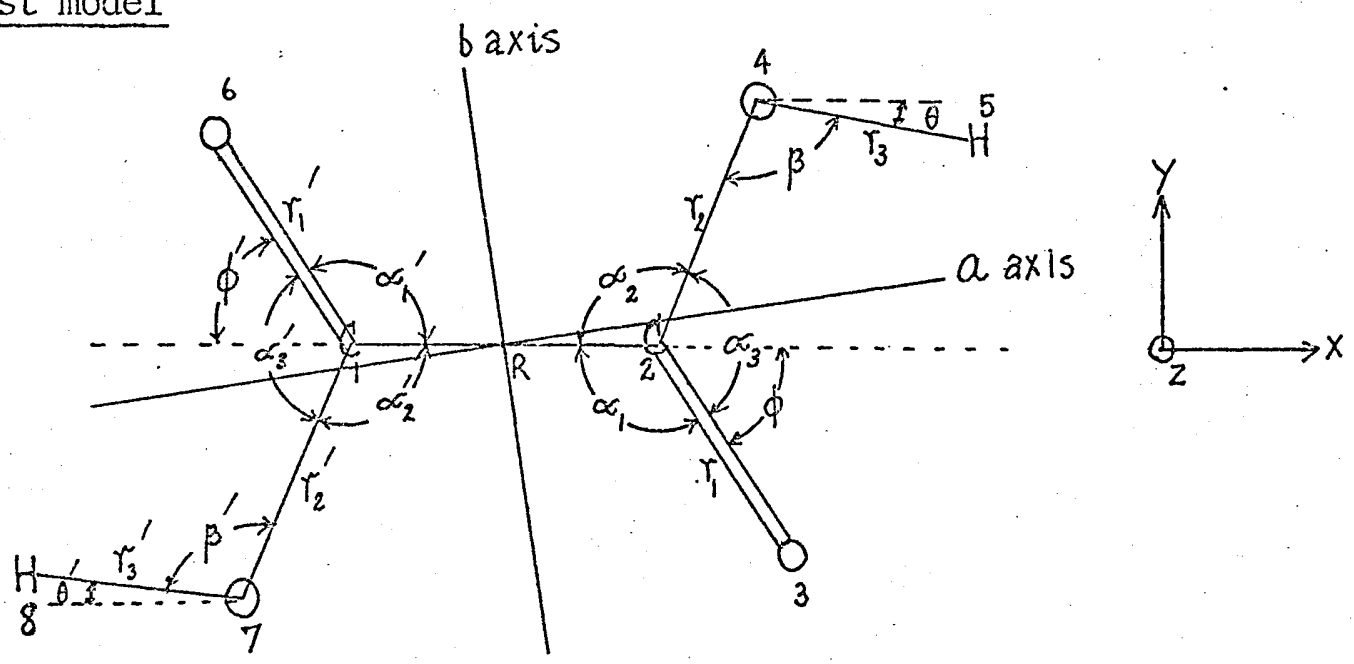

Fig. 17

Gaseous oxalic acid lst model.

Let the oxalic acid molecule be in the $x y$ plane in which the origin is at the centre of the $\mathrm{C}-\mathrm{C}$ bond and the $\mathrm{x}$ axis coincides with this bond.

Using molecular parameters given by Nahlovska, Nahlovsky and Strand (78) we have

$$
\begin{aligned}
& C-C=R=1.548 \mathrm{~A} \\
& C=0=r_{1}=r_{1}^{\prime}=1.208 \mathrm{~A} \\
& C-O=r_{2}=r_{2}^{\prime}=1.339 \mathrm{~A} \\
& 0-H=r_{3}=r_{3}^{\prime}=1.056 \mathrm{~A}
\end{aligned}
$$




$$
\begin{aligned}
\angle C-C=O=a_{1} & =\alpha_{1}^{\prime}=123.1^{\circ} \\
\angle C-C-O=a_{2}=a_{2}^{\prime} & =111.9^{\circ} \\
\angle O-C=O=a_{3}=a_{3}^{\prime} & =125.0^{\circ} \\
C^{\prime} & =\phi^{\prime}=56.9^{\circ} \\
\angle C-O-B=B & =B^{\prime}=104.4^{\circ} \\
\theta & =0^{\prime}=7.5^{\circ}
\end{aligned}
$$

$$
\begin{aligned}
I_{x} & =2 \pi_{0}\left(r_{1} \sin \phi\right)^{2}+2 \pi_{0}\left(r_{2} \sin \left(a_{3}-\phi\right)\right)^{2}+2 \pi_{13}\left(r_{2} \sin \left(a_{3}-\phi\right)-r_{3} \sin \theta\right)^{2} \\
& =84.60248 \text { anu. }(A)^{2}
\end{aligned}
$$

$$
\begin{aligned}
I_{y}^{I} & =2 m_{c}\left(\frac{R}{2}\right)^{2}+2 m_{0}\left(r_{1} \cos \phi+\frac{R}{2}\right)^{2}+2 \pi_{0}\left(r_{2} \cos \left(a_{3}-\phi\right)+\frac{R}{2}\right)^{2} \\
& +2 m_{11}\left(r_{3} \cos \theta+r_{2} \cos \left(\alpha_{3}-\phi\right)+\frac{R}{2}\right)^{2} \\
& =142.31338 \text { ariu. }(A)^{2}
\end{aligned}
$$

$$
\begin{aligned}
I_{z} & =I_{X}+I_{y} \\
& =227.41586 \text { anu. }(\Lambda)^{2}
\end{aligned}
$$

$$
\begin{aligned}
I_{x y}= & -2 n_{0}\left(r_{1} \sin \phi\right)\left(r_{1} \cos \phi+\frac{p}{2}\right)+2 n_{0}\left(r_{2} \sin \left(a_{3}-\phi\right)\right)\left(r_{2} \cos \left(\alpha_{3}-\phi\right)+\frac{R}{2}\right) \\
& +2 m_{1}\left(r_{2} \sin \left(\alpha_{3}-\phi\right)-r_{3} \sin \theta\right)\left(r_{3} \cos 0+r_{2} \cos \left(a_{3}-\phi\right)+\frac{R}{2}\right) \\
= & 9.32566 \text { and }(A)^{2}
\end{aligned}
$$

$$
\begin{aligned}
& I_{x z}=\sum m_{i} x_{i} z_{i}=0 \\
& I_{y z}=\sum m_{i} y_{i} z_{i}=0
\end{aligned}
$$


The matrix $X$ is constructed in wich

$$
X=\left|\begin{array}{ccc}
I_{x} & -I_{x y} & -I_{x z} \\
-I_{y z} & I_{y} & -I_{y z} \\
-I_{z x} & -I_{z y} & I_{z}
\end{array}\right|
$$

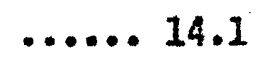

The three roots or the three principal monents of inertia can be determined by solving the deterainantal equation of $X(18)$, thus

$\left|\begin{array}{ccc}84.60248-I & -0.32566 & 0 \\ -9.32566 & 142.81338-1 & 0 \\ 0 & 0 & 227.41586-I\end{array}\right|=0 \quad \ldots \ldots 14.2$

Thus, the three principal noments of inertia are 83.14495 amu. $(A)^{2}$, 144.2709 ams. $(A)^{2}$ and 227.41586 anu. $(A)^{2}$

(b) Principal axes of the gaseous oxalic acid

First rodel

Lot $a, b, c$ bo the three grincipal axes about wihich

$$
\begin{aligned}
& I_{a}=83.14496 \mathrm{amm}(A)^{2} \\
& I_{b}=144.2709 \mathrm{ama} \cdot(A)^{2} \\
& I_{c}=227.41586 \mathrm{ama.}(A)^{2}
\end{aligned}
$$


The natrix $C$ can be diagonalized by an orthogonal matrix $S$ so that

$$
s^{-I_{x}}=\left|\begin{array}{ccc}
I_{a} & 0 & 0 \\
0 & I_{b} & 0 \\
0 & 0 & I_{c}
\end{array}\right|
$$

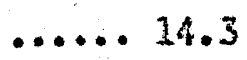

As the oxalic acial is a planar woloculo the problen is easier because the $z$ axis coincides with the $c$ axis and, thus, the matrices $x$ and $S$ can be reduced to second order so that

$$
S^{-I} \gamma=\left|\begin{array}{cc}
I_{a} & 0 \\
0 & I_{b}
\end{array}\right|
$$

$I$ I $I=83.14496$ anu. $(A)^{2}$ and $e_{1}=\frac{1}{\sqrt{1}}\left(i \Omega_{1}+j m_{1}\right)$ then

$$
\left|\begin{array}{ll}
84.60248-83.14406 & -0.32565 \\
-0.32566 & 142.81533-83.14496
\end{array}\right|=0 \quad \ldots \ldots .14 .5
$$

that is

$$
\begin{array}{r}
1.45752 \ell_{1}-9.32565 \mathrm{~m}_{1}=0 \\
-9.32566 \mathrm{~s}_{1}+59.66842=0 \\
\therefore \quad e_{1}=0.983 \mathrm{i}+0.15442 \mathrm{j}
\end{array}
$$


If $I=144.2700 \mathrm{am}(\mathrm{A})^{2} \mathrm{ab} \mathrm{a}_{2}=\frac{1}{\sqrt{1}}\left(\mathrm{is} \mathrm{s}_{2}+j \mathrm{~m}_{2}\right)$ then

$$
\left|\begin{array}{cc}
84.60245-104.2700 & -0.32550 \\
-0.32506 & 122.31339-144.2700
\end{array}\right|=0
$$

that is

$$
\begin{array}{r}
-59.660422_{2}-9.32560 \mathrm{H}_{2}=0 \\
-0.325668_{2}-1.45752 \mathrm{~m}_{2}=0 \\
\therefore c_{2}=-0.15442 i+0.983 j
\end{array}
$$

(......14.9

.....1.10 14.10

The transfomation ratrix $S$ can be construeted from $c_{1}$ and $e_{2}$ as show belot

$$
\therefore s=\left|\begin{array}{cc}
0.080 & -0.1542 \\
0.1542 & 0.028
\end{array}\right|
$$

The transfortation watrix here can be mitton as

$$
S=\left|\begin{array}{rr}
\cos \theta & \cdots \sin 0 \\
\sin 0 & \cos 0
\end{array}\right|
$$

On comaring 14.11 and 14.12 we have

$$
\theta=8^{\circ} 55^{\prime}
$$


That is, the $\mathrm{a}$ and $\mathrm{b}$ axes are $8^{\mathrm{O}} 53^{\prime}$ inclined to the $\mathrm{x}$ and $\mathrm{y}$ axes respectively, as shown in Fig. 17.

\subsection{Principal moments of inertia and the principal axes of gaseous}

oxalic acid

Second mode1

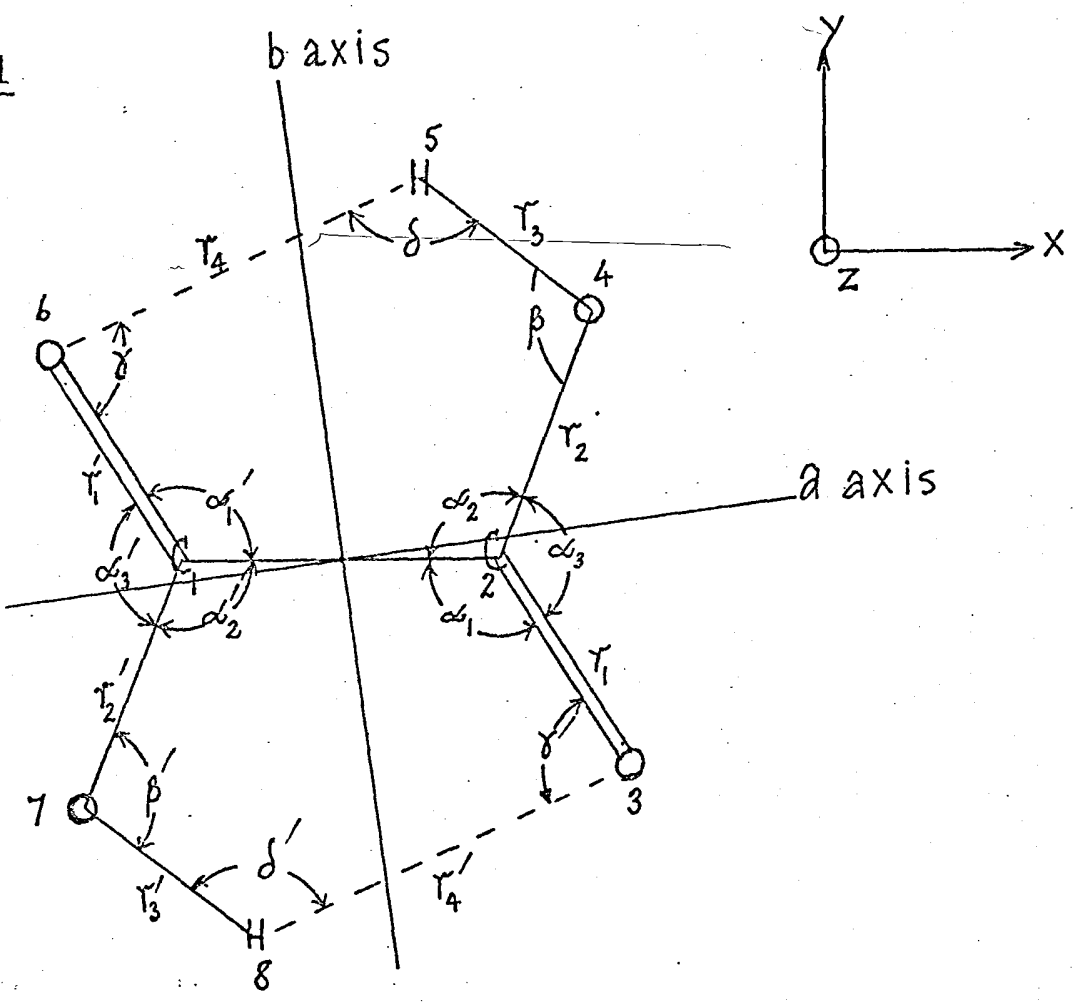

Fig. 18

Gaseous oxalic acid 2nd model

The molecule is in the $x y$ plane in which the C - C bond coincides with the $\mathrm{x}$ axis. Using the molecular parameters given by Nahlovska, Nahlovsky and Strand (78) and the same method as before, the results are

$$
\begin{array}{ll}
I_{a}=88.37968 & \text { amu. }(A)^{2} \\
I_{b}=133.15992 & \text { amu. }(A)^{2} \\
I_{c}=221.5396 & \text { amu. }(A)^{2}
\end{array}
$$


The $c$ axis coincides with the $z$ axis and the and $b$ axes are at $7^{\circ} 26^{\prime} 30^{\prime \prime}$ inclinod to the $x$ and $y$ axes rospectively as shown in Fis. 18. 


\section{CHAPTER 15}

\section{THE G-MATRIX OF THE INPLANE VIBRATIONS}

OF GASEOUS OXALIC ACID IST MODEL

G-matrices for both models were constructed using the method described in chapter 5 .

15.1 Evaluation of the B-matrix of the inplane vibrations

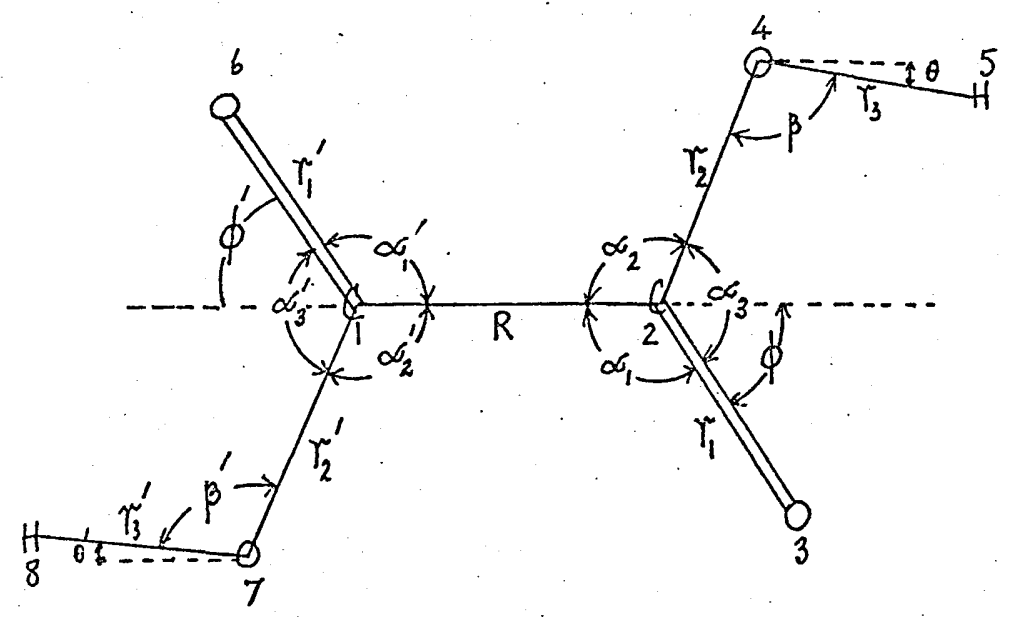

Fig. 19

In the first model of gaseous oxalic acid there are 15

intermal co-ordinates, 7 bond stretching co-ordinates and 8 angle bending co-ordinates, as follows:-

Bond stretching co-ordinates

$$
\begin{aligned}
& \Delta \mathrm{R}=\text { change in } \mathrm{C}-\mathrm{C} \text { distance } \\
& \Delta \mathrm{r}_{1}=\Delta \mathrm{r}_{1}^{\prime}=\text { change in } \mathrm{C}=0 \text { distance } \\
& \Delta \mathrm{r}_{2}=\Delta \mathrm{r}_{2}^{\prime}=\text { change in } \mathrm{C}-0 \text { distance } \\
& \Delta \mathrm{r}_{3}=\Delta \mathrm{r}_{3}^{\prime}=\text { change in } \mathrm{O}-\mathrm{H} \text { distance }
\end{aligned}
$$


Angle bending co-ordinates

$\Delta \alpha_{.}=\Delta \alpha^{2}=$ chance in $\mathrm{C}-\mathrm{C}=0$ ancle Angle bending co-ordinates

$\Delta \mathrm{c}$

$\Delta \alpha_{1}=\Delta \alpha^{\prime}{ }_{1}=$ change in $C-C=0$ angle

$\Delta c$

$\Delta \alpha_{2}=\Delta \alpha_{2}^{\prime}=$ change in $\mathrm{C}-\mathrm{C}-\mathrm{O}$ angle

$\Delta B=\Delta B^{\prime}=$ change in $\mathrm{C}-\mathrm{O}-\mathrm{H}$ angle

For simplicity each atom is labelled by a nunber as shown in Fig. 19 above. B-matrix elements are evaluated using Wilson's $S$ vector method (see section 5.2 ) and the results are:

B-matrix elements of the inplane vibration of gaseous oxalic acid

(1st model)

$$
\text { Since } \begin{aligned}
r_{1} & =r_{1}^{\prime}, r_{2}=r_{2}^{\prime}, r_{3}=r_{3}^{\prime}, \alpha_{1}=\alpha_{1}^{\prime}, \alpha_{2}=\alpha_{2}^{\prime}, \\
\alpha_{3} & =\alpha_{3}^{\prime}, \beta=\beta^{\prime}, \phi=\phi^{\prime}, \theta=\theta^{\prime}
\end{aligned}
$$

the elements of the B-matrix can be written as:-

$$
\begin{array}{ll}
\left(\Delta R, \Delta x_{1}\right)=-1 & \\
\left(\Delta R, \Delta x_{2}\right)=1 & \left(\Delta r_{1}^{\prime}, \Delta x_{1}\right)=\cos \phi \\
\left(\Delta x_{1}, \Delta x_{2}\right)=-\cos \phi & \left(", \Delta y_{1}\right)=-\sin \phi \\
\left(", \Delta y_{2}\right)=\sin \phi & \left(", \Delta x_{6}\right)=-\cos \phi \\
\left(", \Delta x_{3}\right)=\cos \phi & \left(", \Delta y_{6}\right)=\sin \phi \\
\left(", \Delta y_{3}\right)=-\sin \phi & \left(\Delta x_{2}^{\prime}, \Delta x_{1}\right)=\cos \left(\alpha_{3}-\phi\right) \\
\left(\Delta r_{2}, \Delta x_{2}\right)=-\cos \left(\alpha_{3}-\phi\right) & \left(", \Delta y_{1}\right)=\sin \left(\alpha_{3}-\phi\right) \\
\left(", \Delta y_{2}\right)=-\sin \left(\alpha_{3}-\phi\right) & \left(", \Delta x_{7}\right)=-\cos \left(\alpha_{3}-\phi\right) \\
\left(", \Delta x_{4}\right)=\cos \left(\alpha_{3}-\phi\right) & \left(", \Delta y_{7}\right)=-\sin \left(\alpha_{3}-\phi\right) \\
\left(", \Delta y_{4}\right)=\sin \left(\alpha_{3}-\phi\right) & \left({ }^{\prime \prime}\right)
\end{array}
$$




$$
\begin{array}{ll}
\left(\Delta \mathrm{r}_{3}, \Delta \mathrm{x}_{4}\right)=-\cos \theta & \left(\Delta \mathrm{x}_{3}^{\prime}, \Delta \mathrm{x}_{7}\right)=\cos \theta \\
\left(", \Delta \mathrm{y}_{4}\right)=\sin \theta & \left(", \Delta y_{7}\right)=-\sin \theta \\
\left(", \Delta \mathrm{x}_{5}\right)=\cos \theta & \left(", \Delta \mathrm{x}_{8}\right)=-\cos \theta \\
\left(", \Delta y_{5}\right)=-\sin \theta & \left(", \Delta y_{8}\right)=\sin \theta
\end{array}
$$

$\left(r_{1} \Delta a_{1}, \Delta y_{1}\right)=\frac{r_{1}}{R}$

(",$\left.\Delta x_{2}\right)=-\{\sin \Delta\}$

( " , $\left.\Delta y_{2}\right)=-\left\{\frac{r_{1}}{R}+\cos \phi\right\}$

("s,,$\left.\Delta x_{3}\right)=\sin \phi$

$\left(", \Delta y_{3}\right)=\cos \phi$ $\left(r_{2} \Delta \alpha_{2}, \Delta y_{1}\right)=\frac{r_{2}}{R}$

(" $\left." \Delta x_{2}\right)=-\sin \left(\alpha_{3}-\phi\right)$

(" $\left." \Delta y_{2}\right)=\frac{r_{2}}{R}+\cos \left(a_{3}-\phi\right)$

( " , , $\left.\Delta x_{A}\right)=\sin \left(\alpha_{3}-\phi\right)$

( ",$\left.\Delta y_{4}\right)=-\cos \left(\alpha_{3}-\phi\right)$

$$
\begin{aligned}
\left(\sqrt{r_{1} r_{2}} \Delta a a_{3} ; \Delta x_{2}\right) & =\left\{r_{2} \sin \phi+r_{1} \sin \left(\alpha_{3}-\phi\right)\right\} / \sqrt{r_{1} r_{2}} \\
\left(\Delta y_{2}\right) & =\left\{r_{2} \cos \phi-r_{1} \cos \left(r_{3}-\phi\right)\right\} / \sqrt{r_{1} r_{2}}
\end{aligned}
$$$$
\text { ( } \left.\quad, \Delta x_{3}\right)=-r_{2} \sin \phi / \sqrt{r_{1} r_{2}}
$$$$
\text { (c, } \left., \Delta \mathrm{y}_{3}\right)=-\mathrm{r}_{2} \cos 4 / \sqrt{\mathrm{r}_{1} \mathrm{r}_{2}}
$$$$
\text { ( " , } \left., \Delta x_{4}\right)=-r_{1} \sin \left(\alpha_{3}-\phi\right) / \sqrt{r_{1} r_{2}}
$$$$
\text { ( } \left.", \Delta y_{4}\right)=r_{1} \cos \left(\alpha_{3}-\phi\right) / \sqrt{r_{1} r_{2}}
$$

$$
\begin{aligned}
& \left(r_{1}^{\prime} \Delta \alpha_{1}^{\prime}, \Delta x_{1}\right)=\sin \phi \quad\left(r_{2}^{\prime} \Delta \alpha_{2}^{\prime}, \Delta x_{1}\right)=\sin \left(\alpha_{3}-\phi\right) \\
& \text { (" " } \left., \Delta y_{1}\right)=\frac{r_{1}}{R_{1}}+\cos \theta \quad\left(", \Delta y_{1}\right)=-\left\{\frac{r_{2}}{R}+\cos \left(\alpha_{3}-\phi\right)\right\} \\
& \left(", \Delta y_{2}\right)=-\frac{T_{1}}{R} \quad\left(", \Delta y_{2}\right)=\frac{r_{2}}{R} \\
& \text { ( " } \left., \Delta x_{6}\right)=-\sin \quad\left(", \Delta x_{7}\right)=-\sin \left(\alpha_{3}-\phi\right) \\
& \text { (" } \left., \Delta y_{0}\right)=-\cos \phi \quad\left({ }^{n}, \Delta y_{7}\right)=\cos \left(\alpha_{3}-\phi\right)
\end{aligned}
$$




$$
\begin{aligned}
& \left(\sqrt{r^{\prime} I^{\prime}} 2^{\Delta a^{\prime}}, \Delta x_{1}\right)=-\left(r_{2} \sin \phi+r_{1} \sin \left(\alpha_{3}-\phi\right)\right\} / \sqrt{x_{1} T_{2}} \\
& \text { ( } \left.", \Delta y_{3}\right)=\left(r_{1} \cos \left(\alpha_{3}-6\right)-r_{2} \cos 6\right\} / \sqrt{r_{1} r_{2}} \\
& \text { ( " } \left.\quad \Delta x_{3}\right)=r_{2} \sin / \sqrt{r_{1} r_{2}} \\
& \left(\quad " \Delta y_{\theta}\right)=r_{2} \cos \phi / \sqrt{r_{1} r_{2}} \\
& \text { ( } \left.", \Delta x_{7}\right)=r_{1} \sin \left(a_{3}-\phi\right) / \sqrt{r_{1} r_{2}} \\
& \left(\quad, \Delta y_{7}\right)=-r_{1} \cos \left(\alpha_{3}-\frac{6}{7}\right) / \sqrt{r_{1} r_{2}} \\
& \left(\sqrt{r_{2} r_{3}} A B_{j} A x_{2}\right)=-r_{3} \ln \left(\alpha_{3}-4\right) / \sqrt{r_{3} r_{3}} \\
& \text { ( } \left.", \Delta y_{2}\right)=r_{3} \cos \left(a_{3}-\Delta\right) / \sqrt{r_{2} r_{3}} \\
& \left(", \Delta x_{4}\right)=\left\{x_{3} \sin \left(a_{3}-s_{2}\right)-x_{2} \sin \theta\right\} / \sqrt{x_{2} r_{3}} \\
& \text { ( } \left.4, \Delta y_{4}\right)=-\left\{r_{3} \cos \left(\alpha_{3}-\phi\right)+r_{2} \cos \theta\right\} / \sqrt{x_{2} z_{3}} \\
& \left(\because, \Delta x_{5}\right)=r_{2} \operatorname{sine} / \sqrt{r_{2} r_{3}} \\
& \text { ( } \left.. \Delta y_{5}\right)=r_{2} \cos \theta / \sqrt{r_{2} r_{3}} \\
& \left(\sqrt{r_{2} r^{\prime}}{ }_{3} A B, \Delta x_{1}\right)=r_{3} \sin \left(a_{3}-\phi\right) / \sqrt{x_{2} r_{3}} \\
& \text { ( } \left.\quad, \Delta y_{1}\right)=-r_{3} \cos \left(a_{3}-\phi\right) / \sqrt{r_{2} r_{3}} \\
& \text { ( } \left." \quad, \Delta x_{7}\right)=\left\{r_{2} \sin \theta-r_{3} \sin \left(a_{3}-\theta\right)\right\} / \sqrt{r_{2} z_{3}} \\
& \text { ( } \left.\quad, \Delta y_{7}\right)=\left(r_{2} \cos \theta+r_{3} \cos \left(\theta_{3}-\phi\right)\right\} / \sqrt{r_{2} T_{3}} \\
& \left(\|, \Delta x_{8}\right)=-x_{2} \operatorname{sino} \sqrt{r_{2} x_{3}} \\
& \text { ( * , a } \left., \Delta y_{3}\right)=-r_{2} \cos 0 / \sqrt{r_{2} r_{3}}
\end{aligned}
$$

AI the other clonents are zero 
15.2 D-matrix elenents of the inplane vibration of gaseous oxalic acid

(1st model)

$$
\begin{aligned}
& \left(\Delta R, \Delta x_{1}\right)=-\sqrt{\mu_{c}} \\
& \left(\Delta R, \Delta x_{2}\right)=\sqrt{\mu_{c}} \\
& \left(\Delta \mathrm{r}_{1}, \Delta \mathrm{x}_{2}\right)=-\sqrt{\mu_{\mathrm{c}}} \cos \theta \\
& \left(\Delta x_{1}^{\prime}, \Delta x_{1}\right)=\sqrt{\mu_{c}} \cos \phi \\
& \text { (", } \left.\Delta y_{2}\right)=\sqrt{\mu_{c}} \sin \theta \\
& \left(", \Delta y_{1}\right)=-\sqrt{\mu_{c}} \sin \phi \\
& \text { (", } \left., \Delta x_{3}\right)=\sqrt{\mu_{0}} \cos \phi \\
& \text { ( " } \left., \Delta x_{6}\right)=-\sqrt{\mu_{0}} \cos \phi \\
& \text { (" } \left., \Delta y_{3}\right)=-\sqrt{\mu_{0}} \sin \phi \\
& \left(", \Delta y_{6}\right)=\sqrt{\mu_{0}} \sin \phi \\
& \left(\Delta \mathrm{r}_{2}, \Delta \mathrm{x}_{2}\right)=-\sqrt{\mu_{c}} \cos \left(\alpha_{3}-\phi\right) \\
& \left(\Delta r_{2}^{\prime}, \Delta x_{1}\right)=\sqrt{\mu_{c}} \cos \left(\alpha_{3}-\phi\right) \\
& \text { (" } \left., \Delta y_{2}\right)=-\sqrt{\mu_{c}} \sin \left(\alpha_{3}-\phi\right) \\
& \text { ( " } \left., \Delta y_{1}\right)=\sqrt{\mu_{c}} \sin \left(\alpha_{3}-\phi\right) \\
& \text { (", } \left.\Delta x_{4}\right)=\sqrt{\mu_{0}} \cos \left(a_{3}-\phi\right) \\
& \text { ( } \left.", \Delta x_{\eta}\right)=-\sqrt{\mu_{0}} \cos \left(\alpha_{3}-\phi\right) \\
& \text { (", } \left.\Delta y_{4}\right)=\sqrt{\mu_{0}} \sin \left(\alpha_{3}-\frac{\phi}{4}\right) \\
& \text { ( ", } \left., \Delta y_{7}\right)=-i \hat{\mu}_{0} \sin \left(\alpha_{3}-\phi\right) \\
& \left(\Delta \mathrm{T}_{3}, \Delta \mathrm{x}_{4}\right)=-\sqrt{\mu_{0}} \cos 0 \\
& \left(\Delta x^{\prime}{ }_{3}, \Delta x_{7}\right)=\sqrt{u_{0}} \cos \theta \\
& \text { (", } \left.\Delta y_{4}\right)=\sqrt{\mu_{0}} \sin \theta \\
& \text { ( " } \left., \Delta y_{7}\right)=-\sqrt{\mu_{0}} \sin \theta \\
& \text { (" } \left., \Delta x_{5}\right)=\sqrt{\mu_{\mathrm{k}}} \cos \theta \\
& \text { ( } \left.", \Delta x_{\delta}\right)=-\sqrt{\mu_{H}} \cos \theta \\
& \text { (", } \left., \Delta y_{5}\right)=-\sqrt{\mu_{H}} \sin \theta \\
& \left(", y_{B}\right)=\sqrt{\mu_{H}} \sin \theta \\
& \left(r_{1} \Delta \alpha_{1}, \Delta y_{1}\right)=\sqrt{\mu_{c}}\left(r_{1} / R\right) \\
& \left(x_{2} \Delta \alpha_{2}, \Delta y_{1}\right)=-\sqrt{\mu_{c}}\left(r_{2} / R\right) \\
& \text { (" } \left., \Delta x_{2}\right)=-\sqrt{\mu_{c}} \sin \phi \\
& \text { (" } \left., \Delta x_{2}\right)=-\sqrt{\mu_{c}} \sin \left(\alpha_{3}-\phi\right) \\
& \text { (", } \left.\Delta y_{2}\right)=-\sqrt{\mu_{c}}\left\{x_{1} / R+\cos \phi\right\} \\
& \text { (" } \left., \Delta x_{3}\right)=\sqrt{\mu_{0}} \sin \phi \\
& \text { (", } \left., \Delta y_{2}\right)=+\sqrt{\mu_{c}}\left\{r_{2} / R+\cos \left(\alpha_{3}-\phi\right)\right\} \\
& \text { ( } \left.", \Delta y_{3}\right)=\sqrt{u_{0}} \cos \varphi \\
& \text { (" } \left., \Delta x_{4}\right)=\sqrt{\mu_{0}} \sin \left(a_{3}-\phi\right) \\
& \left(", y_{4}\right)=-\sqrt{\mu_{0}} \cos \left(a_{3}-\beta\right)
\end{aligned}
$$




$$
\begin{aligned}
& \left(\sqrt{r_{1} r_{2}} \Delta \sigma_{3}, \Delta x_{2}\right)=\sqrt{\mu_{c}}\left\{r_{2} \sin \phi+r_{1} \sin \left(a_{3}-\phi\right)\right\} / \sqrt{r_{1} r_{2}} \\
& \text { ( " } \left., \Delta y_{2}\right)=\sqrt{\mu_{c}}\left\{r_{2} \cos \phi-r_{1} \cos \left(\alpha_{3}-\phi\right)\right\} / \sqrt{r_{1} r_{2}} \\
& \text { ( } \left.\quad, \Delta x_{3}\right)=-\sqrt{\mu_{0}} r_{2} \sin \phi / \sqrt{r_{1} r_{2}} \\
& \text { ( } \left.", \Delta y_{3}\right)=-\sqrt{\mu_{0}} r_{2} \cos \phi / \sqrt{r_{1} r_{2}} \\
& \text { ( } \left.", \Delta x_{4}\right)=-\sqrt{\mu_{0}} r_{1} \sin \left(\alpha_{3}-\phi\right) / \sqrt{r_{1} r_{2}} \\
& \text { ( } \left., \Delta y_{4}\right)=\sqrt{u_{0}} r_{1} \cos \left(\alpha_{3}-\phi\right) / \sqrt{r_{1} r_{2}} \\
& \left(r^{\prime}{ }_{1} \Delta a_{1}^{\prime}, \Delta x_{1}\right)=\sqrt{\mu_{c}} \sin \phi \\
& \text { (". } \left., \Delta y_{1}\right)={\sqrt{\mu_{c}}}_{c}\left\{r_{1} / R+\cos \phi\right\} \\
& \text { ( " , } \left., \Delta y_{2}\right)=-\sqrt{\mu_{c}}\left(x_{1} / D\right) \\
& \text { ( } \left.", \Delta x_{0}\right)=-\sqrt{\mu_{0}} \sin \theta \\
& \text { (. it, } \left., \Delta y_{6}\right)=-\sqrt{\mu_{0}} \cos \theta \\
& \left(r_{2}^{\prime}{ }_{2}^{\Delta \alpha^{\prime}}{ }_{2}, \Delta x_{1}\right)=\sqrt{u_{c}} \sin \left(\alpha_{3}-\phi\right) \\
& \text { ( ", , } \left.\Delta y_{1}\right)=-\sqrt{\mu_{c}}\left\{r_{2} / R+\cos \left(\alpha_{3}-\phi\right)\right\} \\
& \text { (", } \left., \Delta y_{2}\right)=\sqrt{\mu_{c}}\left(x_{2} / R\right) \\
& \text { (" , } \left.\Delta x_{7}\right)=-\sqrt{u_{0}} \sin \left(\alpha_{5}-\phi\right) \\
& \text { ( } \left.", \Delta y_{7}\right)=\sqrt{1}_{0} \cos \left(\alpha_{3}-\phi\right) \\
& \left(\sqrt{r^{\prime}{ }_{1}{ }^{\prime}{ }_{2}} \Delta a^{\prime}{ }_{3}, \Delta x_{1}\right)=-\sqrt{u_{c}}\left\{r_{2} \sin \phi+r_{1} \sin \left(\alpha_{3}-\phi\right)\right\} / \sqrt{r_{1} r_{2}} \\
& \text { ( } \left.\quad, \Delta y_{1}\right)=\sqrt{\mu_{c}}\left\{r_{1} \cos \left(a_{3}-\phi\right)-x_{2} \cos \phi\right\} / \sqrt{r_{1} r_{2}} \\
& \text { ( " } \left.\quad, \Delta x_{6}\right)=\sqrt{\mu_{0}} r_{2} \sin \phi / \sqrt{r_{1} r_{2}} \\
& \text { ( " } \left.\quad \Delta y_{6}\right)=\sqrt{\mu_{0}} r_{2} \cos \phi / \sqrt{r_{1} r_{2}} \\
& \text { ( } \left.\quad, \Delta x_{7}\right)=\sqrt{\mu_{0}} r_{1} \sin \left(\alpha_{3}-\phi\right) / \sqrt{r_{1} r_{2}} \\
& \text { ( " } \left.\quad \Delta y_{7}\right)=-\sqrt{\mu_{0}} r_{1} \cos \left(\alpha_{3}-\beta\right) / \sqrt{r_{1} r_{2}}
\end{aligned}
$$




$$
\begin{aligned}
& \left(\sqrt{r_{2} r_{3}} \Delta \beta, \Delta x_{2}\right)=-\sqrt{\mu_{c}} r_{3} \sin \left(\alpha_{3}-\phi\right) / \sqrt{r_{2} r_{3}} \\
& \text { ( " } \left., \Delta y_{2}\right)=\sqrt{y_{c}} r_{3} \cos \left(\alpha_{3}-\phi\right) / \sqrt{r_{2} r_{3}} \\
& \text { (", } \left.\Delta x_{4}\right)=\sqrt{\mu_{0}}\left\{r_{3} \sin \left(\alpha_{3}-\phi\right)-r_{2} \sin \theta\right\} / \sqrt{r_{2} r_{3}} \\
& \text { ( } \left." \Delta, \Delta y_{4}\right)=-\sqrt{\mu_{0}}\left\{r_{3} \cos \left(\alpha_{3}-\phi\right)+r_{2} \cos 0\right\} / \sqrt{r_{2} r_{3}} \\
& \text { ( " } \left., \Delta x_{5}\right)=\sqrt{u_{11}} r_{2} \sin \theta / \sqrt{r_{2} r_{3}} \\
& \text { ( " } \left., \Delta y_{5}\right)=\sqrt{\mu_{11}} r_{2} \cos 0 / \sqrt{r_{2} r_{3}}
\end{aligned}
$$

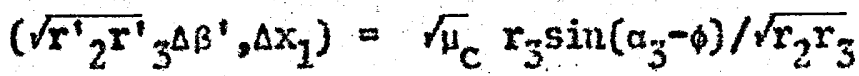

$$
\begin{aligned}
& \text { ( } \left.\quad, \Delta y_{1}\right)=-\sqrt{\mu_{c}} r_{3} \cos \left(\alpha_{3}-\phi\right) / \sqrt{r_{2} r_{3}} \\
& \text { ( " } \left.\quad, \Delta x_{7}\right)=\sqrt{\mu_{0}}\left\{r_{2} \sin \theta-r_{3} \sin \left(\alpha_{3}-\phi\right)\right\} / \sqrt{r_{2} r_{3}} \\
& \text { (". , } \left.\Delta y_{7}\right)=\sqrt{\mu_{0}}\left\{r_{2} \cos \theta+r_{3} \cos \left(\alpha_{3}-\phi_{0}\right)\right\} / \sqrt{r_{2} r_{3}} \\
& \left.r \quad ", \Delta x_{8}\right)=-\sqrt{\mu_{11}} r_{2} \sin \theta / \sqrt{r_{2} x_{3}} \\
& \text { ( } \left." ., \Delta y_{8}\right)=-\sqrt{\mu_{14}} r_{2} \cos \theta / \sqrt{r_{2} r_{3}}
\end{aligned}
$$

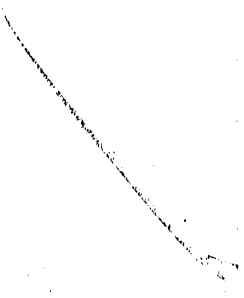

All the other eloments are zero

$$
\begin{aligned}
& \mu_{c}=\frac{1}{m_{c}} \\
& \mu_{0}=\frac{1}{n_{0}} \\
& \mu_{H}=\frac{1}{n_{H}}
\end{aligned}
$$

where $\mathrm{n}_{\mathrm{c}}, \mathrm{m}_{\mathrm{o}}, \mathrm{n}_{i}$ are the masses of corbon, oxygen and hydrogen atoms rospectively. 
15.3 G-matrix elenents in internal co-ordinates of the inplane vibration of gassous oxalic acid (1st model)

$$
\begin{aligned}
& G(1,1)=2 \mu_{c} \\
& G(1,2)=-\mu_{c} \cos \phi \\
& G(1,3)=-\mu_{c} \cos \phi \\
& G(1,4)=-\mu_{c} \cos \left(\alpha_{3}-\phi\right) \\
& G(1,5)=-\mu_{c} \cos \left(\alpha_{3}-\phi\right) \\
& G(1,6)=0 \\
& G(1,7)=0 \\
& G(1,8)=-\mu_{c} \sin \phi \\
& G(1,9)=-\mu_{c} \sin \left(a_{3}-\phi\right) \\
& G(1,10)=\mu_{c}\left\{r_{2} \sin \phi+r_{1} \sin \left(\alpha_{3}-\phi\right)\right\} / \sqrt{r_{1} r_{2}} \\
& G(1,11)=-\mu_{c} \sin \phi \\
& G(1,12)=-\mu_{c} \sin \left(\alpha_{3}-\phi\right) \\
& G(1,13)=\mu_{c}\left\{r_{2} \sin \phi+r_{1} \sin \left(\alpha_{3}-\phi\right)\right\} / \sqrt{r_{1} r_{2}} \\
& G(1,14)=-\mu_{c} r_{3} \sin \left(\alpha_{3}-\phi\right) / \sqrt{r_{2} r_{3}} \\
& G(1,15)=-\mu_{c} r_{3} \sin \left(\alpha_{3}-\phi\right) / \sqrt{r_{2} r_{3}} \\
& G=-\mu_{c} \cos \phi \\
& G(2,5)=\mu_{c}+\mu_{0} \cos \alpha_{3} \\
& G(2,1)=0 \\
& G(2,2)=0 \\
& G(2,3)=0 \\
& G(2,4)=0
\end{aligned}
$$




$$
\begin{aligned}
& \mathrm{G}(2,8)=-\mu_{\mathrm{c}} \frac{s_{1}}{k} \sin \phi \\
& G(2,9)=u_{c} \frac{r_{2}}{R} \sin +u_{c} \sin \alpha_{3} \\
& G(2,10)=-\mu_{c} r_{1} \sin \alpha_{3} / \sqrt{r_{1} r_{2}} \\
& G(2,11)=-\mu_{c} \frac{r_{1}}{R} \sin \theta \\
& G(2,12)=\mu_{c} \frac{x_{2}}{k} \sin \phi \\
& G(2,13)=0 \\
& G(2,14)=\mu_{c} r_{3} \sin \alpha_{3} / \sqrt{r_{2} r_{3}} \\
& G(2,15)=0 \\
& G(3,1)=-\mu_{c} \cos \phi \\
& G(3,2)=0 \\
& G(3,3)=\mu_{c}+\mu_{0} \\
& G(3,4)=0 \\
& G(3,5)=\mu_{c} \cos \alpha_{3} \\
& G(3,6)=0 \\
& G(3,7)=0 \\
& G(3,8)=-\mu c_{1} \frac{r_{1}}{R} \sin \phi \\
& G(5,9)-\mu_{c} \frac{r_{2}}{R} \sin \phi \\
& G(3,10)=0 \\
& G(3,11)--\mu_{c} \frac{r_{1}}{R} \sin \phi \\
& G(3,12)=\mu_{c} \frac{r_{2}}{R} \sin \phi+\mu_{c} \sin \alpha_{3} \\
& G(3,13)=-\mu_{c} r_{1} \operatorname{sina}_{3} / \sqrt{r_{1} r_{2}} \\
& G(3,14)=0 \\
& G(3,15)=\mu_{c} r_{3} \sin \alpha_{3} / \sqrt{x_{2} x_{3}}
\end{aligned}
$$


- 117 -

$$
\begin{aligned}
& G(4,1)=-\mu_{c} \cos \left(\alpha_{3}-\phi\right) \\
& \mathrm{G}(4,2)=\mu_{c} \cos \alpha_{3} \\
& G(4,3)=0 \\
& G(4,4)=\mu_{C}+\mu_{0} \\
& \mathrm{G}(4,5)=0 \\
& G(4,0)=+p_{0} \cos \beta \\
& G(4,7)=0 \\
& G(4,8)-\mu_{c} \frac{r_{1}}{R_{r_{2}}} \sin \left(\alpha_{3}-\phi\right)+\mu_{c} \sin \left(\alpha_{3}\right) \\
& G(4,9)=-\mu_{c} \frac{r_{2}}{R} \sin \left(\alpha_{3}-\phi\right) \\
& G(4,10)=-\mu_{c_{1}} x_{2} \sin \alpha_{3} / \sqrt{r_{1} r_{2}} \\
& G(4,11)=\mu_{c} \frac{r_{1}}{R_{r_{2}}} \sin \left(\alpha_{3}-4\right) \\
& G(4,12)=-\mu_{c} \frac{r_{2}}{R} \sin \left(a_{3}-\hat{\varphi}\right) \\
& G(4,13)=0 \\
& G(4,14)-\mu_{0} r_{2} \sin 8 / \sqrt{r_{2} r_{3}} \\
& G(4,15)=0 \\
& G(5,1) \quad-\mu_{c} \cos \left(a_{3}-\phi\right) \\
& G(5,2)=0 \\
& G(5,3)=\mu_{c} \cos \alpha_{3} \\
& G(5,4)=0 \\
& G(5,5)=\mu_{c}+\mu_{0} \\
& G(5,6)=0 \\
& G(5,7)=\mu_{0} \cos B \\
& G(5,8) \quad \mu_{c} \frac{r_{1}}{r_{r_{2}}} \sin \left(\alpha_{3}-\phi\right) \\
& G(5,9)=-\mu_{c} \frac{2}{R} \sin \left(\alpha_{3}-\phi\right) \\
& \mathrm{G}(5,10)=0 \\
& G(5,11)=\mu_{c} \frac{r_{1}}{R} \sin \left(a_{3}-\phi\right)+\mu_{c} \sin a_{3}
\end{aligned}
$$


$-118-$

$$
\begin{aligned}
& G(5,12)=-\mu_{c} \frac{r_{2}}{R} \sin \left(\alpha_{3}-\phi\right) \\
& G(5,13)=-\mu_{c} r_{2} \sin \alpha_{3} / \sqrt{r_{1} r_{2}} \\
& G(5,14)=0 \\
& G(5,15)=-\mu_{0} r_{2} \sin \beta / \sqrt{r_{2} r_{3}}
\end{aligned}
$$

$$
\begin{aligned}
& G(6,1)=0 \\
& G(6,2)=0 \\
& G(6,3)=0 \\
& G(6,4)=\mu_{0} \cos B \\
& G(6,5)=0 \\
& G(6,6)=\mu_{0}+\mu_{11} \\
& G(6,7)=0 \\
& G(6,8)=0 \\
& G(6,9)=-\mu_{0} \sin \\
& G(6,10)=\mu_{0} r_{1} \sin \beta / \sqrt{r_{1} r_{2}} \\
& G(6,11)=0 \\
& G(6,12)=0 \\
& G(6,13)=0 \\
& G(6,14)=-\mu_{0} r_{3} \sin \beta / \sqrt{r_{2} r_{3}} \\
& G(6,15)=0
\end{aligned}
$$

$$
\begin{aligned}
& G(7,1)=0 \\
& G(7,2)=0 \\
& G(7,5)=0 \\
& G(7,4)=0
\end{aligned}
$$




$$
\begin{aligned}
& G(7,5) \quad-\mu_{0} \cos B \\
& G(7,0)=0 \\
& G(7,7)=\mu_{0}+H_{H} \\
& G(7,8)=0 \\
& G(7,9)=0 \\
& G(7,10)=0 \\
& G(7,11)=0 \\
& G(7,12)=-\mu_{0} \sin B \\
& G(7,13)=\mu_{0} r_{1} \sin \beta / \sqrt{r_{1} r_{2}} \\
& G(7,14)=0 \\
& G(7,15)--\mu_{0} r_{3} \sin \beta / \sqrt{r_{2} r_{3}} \\
& G(8,1)=-\mu_{c} \sin \\
& \mathrm{G}(8,2)=-\mu_{\mathrm{c}} \frac{\mathrm{r}_{1}}{\mathrm{R}_{1}} \sin \phi \\
& G(8,3)=-\mu_{c} \frac{r_{1}}{R} \sin \phi \\
& G(8,4)=\mu_{c} \frac{r_{1}}{\beta} \sin \left(a_{3}-\phi\right)+\mu_{c} \sin \alpha_{3} \\
& G(8,5)=\mu_{c} \frac{1}{R} \sin \left(\alpha_{3}-\phi\right) \\
& G(8,6)=0 \\
& G(8,7)=0 \\
& G(8,8)=\mu_{c}\left\{2 \frac{r_{1}}{R^{2}}+2 \frac{r_{1}}{R} \cos \phi+1\right\}+\mu_{0} \\
& G(8,9)=-2 \mu_{c}\left(r_{1} r_{2} / R^{2}\right)-\mu_{c} \frac{r_{1}}{R} \cos \left(\alpha_{3}-\phi\right)-\mu_{c} \frac{r_{2}}{R} \cos \phi-\mu_{c} \cos \alpha_{3} \\
& G(8,10)=\left\{u_{c}\left(r_{1}{ }^{2} / R\right) \cos \left(\alpha_{3}-\phi\right)+u_{c} r_{1} \cos \alpha_{3}-\mu_{c}\left(r_{1} r_{2} / R\right) \cos \phi\right. \\
& \left.-\mu_{c} r_{2}-\mu_{0} r_{2}\right\} / \sqrt{r_{1} x_{2}} \\
& G(8,11)=2 u_{c}\left(r_{1}^{2} / R^{2}\right)+2 u_{c}\left(r_{1} / R\right) \cos \phi \\
& G(8,12)=-2 \mu_{c}\left(r_{1} r_{2} / R^{2}\right)-\mu_{c}\left(r_{1} / R\right) \cos \left(\alpha_{3}-\phi\right)-\mu_{c}\left(r_{2} / R\right) \cos \phi
\end{aligned}
$$




$$
\begin{aligned}
& G(8,13)=\left\{\mu_{c}\left(r_{1}{ }^{2} / R\right) \cos \left(a_{3}-\phi\right)-\mu_{c}\left(r_{1} r_{2} / R\right) \cos \phi\right\} / \sqrt{r_{1} r_{2}} \\
& G(8,14)=\left\{-\mu_{c} r_{3} \cos \alpha_{3}-\mu_{c}\left(r_{1} r_{3} / R\right) \cos \left(\alpha_{3}-\phi\right)\right\} / \sqrt{r_{2} r_{3}} \\
& G(8,15)=-\mu_{c}\left(r_{1} r_{3} / R\right) \cos \left(\alpha_{3}-\phi\right) / \sqrt{r_{2} r_{3}} \\
& G(9,1)--u_{c} \sin \left(\alpha_{3}-\phi\right) \\
& G(9,2)-\mu_{c} \frac{r_{2}}{R} \sin \phi+\mu_{c} \sin \alpha_{3} \\
& G(\theta, 3)=\mu_{c}\left(r_{2} / R\right) \sin \\
& G(9,4)-\mu_{c}\left(r_{2} / R\right) \sin \left(\alpha_{3}-\phi\right) \\
& G(9,5)=-\mu_{c}\left(r_{2} / R\right) \sin \left(a_{3}-\phi\right) \\
& G(9,6)=-\mu_{0} \sin B \\
& G(9,7)=0 \\
& G(9,8)=-2 u_{c}\left(r_{1} r_{2} / R^{2}\right)-\mu_{c}\left(r_{1} / R\right) \cos \left(r_{3}-\phi\right)-\mu_{c}\left(r_{2} / R\right) \cos \phi \\
& -\mu_{c} \cos \alpha_{3} \\
& G(9,9)=\mu_{c}\left\{\left(2 r_{2}^{2} / R^{2}\right)+\left(2 r_{2} / R\right) \cos \left(\alpha_{3}-\phi\right)+1\right\}+\omega_{0} \\
& G(9,10)=\left\{\mu_{c}\left(r_{2}{ }^{2} / R\right) \cos \phi+\mu_{c} r_{2} \cos \alpha_{3}-\mu_{c}\left(r_{1} r_{2} / R\right) \cos \left(\alpha_{3}-\phi\right)\right. \\
& \left.-\mu_{c} r_{1}-\mu_{0} r_{1}\right\} / \sqrt{r_{1} r_{2}} \\
& G(9,11)=-2 \mu_{c}\left(r_{1} r_{2} / R^{2}\right)-\mu_{c}\left(r_{1} / R\right) \cos \left(\alpha_{3}-\phi\right)-\mu_{c}\left(r_{2} / R\right) \cos \phi \\
& G(0,12)=2 \mu_{c}\left(r_{2}^{2} / R^{2}\right)+2 \mu_{c}\left(r_{2} / R\right) \cos \left(a_{3}-\phi\right) \\
& G(0,13)=\left\{\mu_{c}\left(r_{2}^{2} / R\right) \cos \phi-\mu_{c}\left(r_{1} r_{2} / R\right) \cos \left(\alpha_{3}-\phi\right)\right\} / \sqrt{r_{1} r_{2}} \\
& G(9,14)=\left\{\mu_{c}\left(r_{2} r_{3} / R\right) \cos \left(a_{3}-\phi\right)+\mu_{c} r_{3}+\mu_{0} r_{3}-\mu_{o} r_{2} \cos \beta\right\} \\
& \sqrt{r_{2} r_{3}} \\
& G(9,15)=\mu_{c}\left(\sqrt{r_{2^{x}}} / R\right) \cos \left(\alpha_{3}-\phi\right)
\end{aligned}
$$




$$
\begin{aligned}
& G(10,1)=\mu_{c}\left\{r_{2} \sin \phi+r_{1} \sin \left(\alpha_{3}-\phi\right)\right\} / \sqrt{r_{1} x_{2}} \\
& G(10,2)=-\mu_{c} r_{1} \sin \alpha_{3} / \sqrt{r_{1} r_{2}} \\
& G(10,3)=0 \\
& G(10,4)=\mu_{c} r_{2} \operatorname{sina}_{3} / \sqrt{r_{1} r_{2}} \\
& G(10,5)=0 \\
& G(10,6)=H_{0} r_{1} \sin \beta / \sqrt{r_{1} x_{2}} \\
& G(10,7)=0 \\
& G(10,8)=\left(u_{c}\left(r_{1}{ }^{2} / R\right) \cos \left(\alpha_{3}-\phi\right)+u_{c} r_{1} \cos \alpha_{3}-u_{c}\left(r_{1} r_{2} / R\right) \cos \phi\right. \\
& \left.-\mu_{c} r_{2}-\mu_{o} r_{2}\right\} / \sqrt{r_{1} r_{2}} \\
& G(10,9)=\left\{u_{c}\left(r_{2}{ }^{2} / R\right) \cos \phi+u_{c} r_{2} \cos a_{3}-u_{c}\left(r_{1} r_{2} / R\right) \cos \left(a_{3}-\phi\right)\right. \\
& -\mu_{c} x_{1}-\mu_{0} r_{1} J / \sqrt{r_{1} r_{2}} \\
& \left.G(10,10)=\mu_{c}\left(r_{1} / r_{2}\right)+\left(r_{2} / r_{1}\right)-2 \cos a_{3}\right\}+\mu_{0}\left\{\left(r_{1} / r_{2}\right)+\left(r_{2} / r_{1}\right\}\right. \\
& G(10,11)=\left\{\mu_{c}\left(r_{1}{ }^{2} / R\right) \cos \left(\alpha_{3}-\phi\right)-\mu_{c}\left(r_{1} r_{2} / R\right) \cos \phi\right\} / \sqrt{r_{1} r_{2}} \\
& \left.G(10,12)=t \mu_{c}\left(r_{2}^{2} / R\right) \cos \theta-\mu_{c}\left(r_{1} r_{2} / R\right) \cos \left(a_{3}-\phi\right)\right\} / \sqrt{r_{1} r_{2}} \\
& G(10,13)=0 \\
& G(10,14)=\left\{\mu_{c} x_{3} \cos \alpha_{3}-u_{c}\left(x_{1} r_{3} / r_{2}\right)-\mu_{0}\left(r_{1} r_{3} / r_{2}\right)+u_{0} x_{1} \cos \beta\right\} \\
& \sqrt{r_{1} r_{3}} \\
& G(10,15)=0 \\
& G(11,1)=-\mu_{c} \sin \phi \\
& G(11,2)=-\mu_{c}\left(r_{1} / R\right) \sin \phi \\
& G(11,3)=-\mu_{c}\left(r_{1} / R\right) \sin \phi \\
& G(11,4)=\mu_{c}\left(r_{1} / R\right) \sin \left(\alpha_{3}-\phi\right) \\
& G(11,5)=\mu_{c}\left(r_{1} / R\right) \sin \left(\alpha_{3}-\phi\right)+\mu_{c} \sin a_{3} \\
& G(11,6)=0 \\
& G(11,7)=0
\end{aligned}
$$




$$
\begin{aligned}
& G(11,8)=2 \mu_{c}\left(r_{1} 2 / R^{2}\right)+2 \mu_{c}\left(r_{1} / R\right) \cos \phi \\
& G(11,9)=-2 \mu_{c}\left(r_{1} r_{2} / R^{2}\right)-\mu_{c}\left(r_{1} / R\right) \cos \left(\alpha_{3}-\phi\right)-\mu_{c}\left(r_{2} / R\right) \cos \phi \\
& G(11,10)=\left\{\mu_{c}\left(r_{1}^{2} / R\right) \cos \left(\alpha_{3}-\phi\right)-\mu_{c}\left(r_{1} r_{2} / R\right) \cos \phi\right\} / \sqrt{r_{1} r_{2}} \\
& G(11,11)=\mu_{c}\left\{2\left(r_{1}^{2} / R^{2}\right)+2\left(r_{1} / R\right) \cos \phi+1\right\}+\mu_{0} \\
& G(11,12)=-2 u_{c}\left(r_{1} r_{2} / R^{2}\right)-\mu_{c}\left(r_{1} / R\right) \cos \left(\alpha_{3}-\phi\right)-\mu_{c}\left(r_{2} / R\right) \cos \phi \\
& -\mu_{c} \cos \alpha_{3} \\
& G(11,13)=\left\{\mu_{c}\left(r_{1}^{2} / R\right) \cos \left(\alpha_{3}-\phi\right)+\mu_{c} r_{1} \cos \alpha_{3}-\mu_{c}\left(r_{1} r_{2} / R\right) \cos \phi\right. \\
& \left.-\mu_{c} x_{2}-\mu_{o} x_{2}\right\} / \sqrt{r_{1} r_{2}} \\
& G(11,14)=-u_{c}\left(r_{1} r_{3} / R\right) \cos \left(a_{3}-\phi\right) / \sqrt{r_{2} r_{3}} \\
& G(11,15)=t \mu_{c}\left(r_{1} r_{3} / R\right) \cos \left(a_{3}-\phi\right)-\mu_{c} r_{3} \cos \alpha_{3} l / \sqrt{r_{2} r_{3}} \\
& G(12,1)=-\mu_{c} \sin \left(\alpha_{3}-\phi\right) \\
& G(12,2)=\mu_{c}\left(r_{2} / R\right) \sin \phi \\
& G(12,3)=u_{c}\left(r_{2} / R\right) \sin \phi+u_{c} \sin a_{3} \\
& G(12,4)=-\mu_{c}\left(r_{2} / R\right) \sin \left(a_{3}-\phi\right) \\
& G(12,5)=-\mu_{c}\left(r_{2} / R\right) \sin \left(a_{3}-\phi\right) \\
& G(12,6)=0 \\
& G(12,7)=-\mu_{0} \sin B \\
& G(12,8)=-2 \mu_{c}\left(r_{1} x_{2} / R^{2}\right)-\mu_{c}\left(r_{1} / R\right) \cos \left(a_{3}-\phi\right)-\mu_{c}\left(x_{2} / R\right) \cos \phi \\
& G(12,9)=2 \mu_{c}\left(r_{2}{ }^{2} / R^{2}\right)+2 \mu_{c}\left(r_{2} / R\right) \cos \left(a_{3}-\phi\right) \\
& G(12,10)=\left\{\mu_{c}\left(r_{2}{ }^{2} / R\right) \cos \phi-\mu_{c}\left(r_{1} r_{2} / R\right) \cos \left(\alpha_{3}-\phi\right)\right\} / \sqrt{r_{1} r_{2}} \\
& G(12,11)=-2 \mu_{c}\left(r_{1} r_{2} / R^{2}\right)-\mu_{c}\left(r_{1} / R\right) \cos \left(\alpha_{3}-\phi\right)-\mu_{c}\left(r_{2} / R\right) \cos \beta-\mu_{c} \cos \alpha_{3} \\
& G(12,12)=\mu_{c}\left\{2 \frac{x_{2}^{2}}{R^{2}}+2 \frac{r_{2}}{K} \cos \left(\alpha_{3}-\phi\right)+1\right\}+\mu_{0} \\
& G(12,13)=l_{c} r_{2}{ }^{2} \cos a_{3}+\mu_{c}\left(r_{2}^{2} / R\right) \cos \phi-\mu_{c}\left(r_{1} r_{2} / R\right) \cos \left(a_{3}-\phi\right) \\
& \left.-\mu_{c} r_{1}-\mu_{0} r_{1}\right\} / \sqrt{r_{1} r_{2}} \\
& G(12,14)=u_{c}\left(\sqrt{r_{2} r_{3}} / R\right) \cos \left(\alpha_{3}-\phi\right) \\
& G(12,15)=\left\{\mu_{c}\left(r_{2} r_{3} / R\right) \cos \left(a_{3}-\phi\right)+\mu_{c} r_{3}+\mu_{o} r_{3}-\mu_{o} r_{2} \cos \beta\right\} / \sqrt{r_{2} r_{3}}
\end{aligned}
$$




$$
\begin{aligned}
& G(13,1)=u_{c}\left\{r_{2} \sin \phi+r_{1} \sin \left(a_{3}-\phi\right)\right\} / \sqrt{r_{1} r_{2}} \\
& G(13,2)=0 \\
& G(13,3)=-\mu_{c} r_{1} \sin a_{3} / \sqrt{r_{1} x_{2}} \\
& G(13,4)=0 \\
& G(13,5)=-\mu_{c} x_{2} \sin a_{3} / \sqrt{r_{1} r_{2}} \\
& G(13,6)=0 \\
& G(13,7)=H_{0} r_{1} \sin \beta / \sqrt{r_{1} x_{2}} \\
& G(13,8)=\left\{\mu_{c}\left(r_{1}{ }^{2} / R\right) \cos \left(a_{3}-\phi\right)-\mu_{c}\left(r_{1} r_{2} / R\right) \cos \phi\right\} / \sqrt{r_{1} r_{2}} \\
& G(13,9)=\left(u_{c}\left(r_{2}{ }^{2} / R\right) \cos \phi-u_{c}\left(r_{1} r_{2} / R\right) \cos \left(a_{3}-\phi\right)\right\} / \sqrt{r_{1} r_{2}} \\
& \mathrm{G}(13,10)=0 \\
& G(13,11)=f \mu_{c}\left(r_{1}^{2} / R\right) \cos \left(\alpha_{3}-\phi\right)+\mu_{c} r_{1} \cos \alpha_{3}-\mu_{c}\left(r_{1} r_{2} / R\right) \cos \phi \\
& \left.-\mu_{c} x_{2}-\mu_{0} x_{2}\right\} / \sqrt{r_{1} r_{2}} \\
& G(13,12)=\left[\mu_{c} r_{2} \cos \alpha_{3}+\mu_{c}\left(r_{2}{ }^{2} / R\right) \cos \phi-\mu_{c}\left(r_{1} r_{2} / R\right) \cos \left(\alpha_{3}-\phi\right)\right. \\
& \left.-\mu_{c} r_{1}-\mu_{0} r_{1}\right\} / \sqrt{r_{1} r_{2}} \\
& G(13,13)=\mu_{c}\left\{\left(r_{1} / r_{2}\right)+\left(r_{2} / r_{1}\right)-2 \cos \alpha_{3}\right\}+\mu_{0}\left\{\left(r_{1} / r_{2}\right)+\left(r_{2} / r_{1}\right)\right\} \\
& G(13,14)=0 \\
& G(13,15)=\left\{u_{c} r_{3} \cos \alpha_{3}-\mu_{c}\left(r_{1} r_{3} / r_{2}\right)-\mu_{0}\left(r_{1} r_{3} / r_{2}\right)+\mu_{0} r_{1} \cos \beta\right\} \\
& / \sqrt{r_{1} r_{3}}
\end{aligned}
$$

$$
\begin{aligned}
& G(14,1)=-\mu_{c} r_{3} \sin \left(\alpha_{3}-\phi\right) / \sqrt{r_{2} r_{3}} \\
& G(14,2)=\mu_{c} r_{3} \sin \alpha_{3} / \sqrt{r_{2} r_{3}} \\
& G(14,3)=0 \\
& G(14,4)=-\mu_{0} r_{2} \sin \beta / \sqrt{r_{2} r_{3}} \\
& G(14,5)=0 \\
& G(14,0)=-\mu_{0} r_{3} \sin \beta / \sqrt{r_{2} r_{3}} \\
& G(14,7)=0
\end{aligned}
$$




$$
\begin{aligned}
& G(14,8)=\left\{-\mu_{c} r_{3} \cos a_{3}-\mu_{c}\left(r_{1} r_{3} / R\right) \cos \left(a_{3}-\phi\right)\right\} / \sqrt{r_{2} r_{3}} \\
& G(14,9)=\left\{\mu_{c}\left(r_{2} r_{3} / R\right) \cos \left(\alpha_{3}-\phi\right)+\mu_{c} r_{3}+\mu_{0} r_{3}-\mu_{0} r_{2} \cos \beta\right\} / \sqrt{r_{2} r_{3}} \\
& G(14,10)=\left\{\mu_{c} x_{3} \cos \alpha_{3}-\mu_{c}\left(r_{1} r_{3} / r_{2}\right)-u_{0}\left(r_{1} r_{3} / r_{2}\right)+\mu_{o} r_{1} \cos \beta\right\} / \sqrt{r_{1} r_{3}} \\
& G(14,11)=-\mu_{c}\left(r_{1} r_{3} / R\right) \cos \left(a_{3}-\phi\right) / \sqrt{r_{2} r_{3}} \\
& G(14,12)=\mu_{c}\left(\sqrt{r_{2} r_{3}} / R\right) \cos \left(a_{3}-\phi\right) \\
& G(14,13)=0 \\
& G(14,14)=\mu_{c}\left(r_{3} / r_{2}\right)+\mu_{0}\left\{\left(r_{2} / r_{3}\right)+\left(r_{3} / r_{2}\right)-2 \cos \beta\right\}+u_{H}\left(r_{2} / r_{3}\right) \\
& G(14,15)=0 \\
& G(15,1)=-w_{c} r_{3} \sin \left(\alpha_{3}-\phi\right) / \sqrt{r_{2} r_{3}} \\
& G(15,2)=0 \\
& G(15,3)=u_{c} r_{3} \sin \alpha_{3} / \sqrt{r_{2} r_{3}} \\
& G(15,4)=0 \\
& G(15,5)=-\mu_{0} r_{2} \sin \beta / \sqrt{r_{2} r_{3}} \\
& G(15,0)=0 \\
& G(15,7)=-\mu_{0} T_{3} \sin \beta / \sqrt{r_{2} r_{3}} \\
& G(15,8)=-\mu_{c}\left(r_{1} r_{3} / R\right) \cos \left(a_{3}-\phi\right) / \sqrt{r_{2} r_{3}} \\
& G(15,9)=\mu_{c}\left(\sqrt{x_{2} x_{3}} / R\right) \cos \left(\alpha_{3}-\phi\right) \\
& G(15,10)=0 \\
& G(15,21)=\left\{-u_{c}\left(r_{1} r_{3} / R\right) \cos \left(a_{3}-\phi\right)-u_{c} r_{3} \cos \alpha_{3}\right\} / \sqrt{r_{2} r_{3}} \\
& G(15,12)=\left\{\mu_{c}\left(r_{2} r_{3} / R\right) \cos \left(a_{3}-\phi\right)+u_{c} r_{3}+u_{0} r_{3}-u_{0} r_{2} \cos B\right\} / \sqrt{r_{2} r_{3}} \\
& G(15,13)=\left\{\mu_{c} r_{3} \cos \alpha_{3}-\mu_{c}\left(r_{1} r_{3} / r_{2}\right)-\mu_{0}\left(r_{1} r_{3} / r_{2}\right)+\mu_{0} r_{1} \cos \beta\right\} / \sqrt{x_{1} r_{3}} \\
& G(15,14)=0 \\
& G(15,15) \Rightarrow \mu_{c}\left(r_{3} / r_{2}\right)+\mu_{0}\left\{\left(r_{2} / r_{3}\right)+\left(r_{3} / r_{2}\right)-2 \cos \theta\right\}+\mu_{H}\left(r_{2} / r_{3}\right)
\end{aligned}
$$


15.4 Symatry co-ordinates of the inplane vibration of gaseous

oxalic acid lst model

The transformation tablo of the internal co-ordinates of

oxalic acid 1st model is given below.

Table 12

\begin{tabular}{|c|c|c|c|c|}
\hline$c_{2 h}$ & $\mathbf{E}$ & $\mathrm{c}_{2}$ & $i$ & $\sigma_{\mathrm{h}}$ \\
\hline$\Delta R$ & $\Delta R$ & $\Delta R$ & $\Delta R$ & $\Delta R$ \\
\hline$\Delta r_{1}$ & $\Delta \mathrm{r}_{1}$ & $\Delta \mathrm{r}_{1}^{\prime}$ & $\Delta r_{1}^{\prime}$ & $\Delta r_{1}$ \\
\hline$\Delta r_{1}^{\prime}$ & $\Delta r^{\prime}{ }_{1}$ & $\Delta r_{1}$ & $\Delta r_{1}$ & $\Delta r_{1}^{\prime}$ \\
\hline$\Delta \mathrm{r}_{2}$ & $\Delta r_{2}$ & $\Delta \mathrm{r}^{\prime}{ }_{2}$ & $\Delta r_{2}^{\prime}$ & $\Delta \mathrm{r}_{2}$ \\
\hline$\Delta r^{\prime}{ }_{2}$ & $\Delta r^{\prime}{ }_{2}$ & $\Delta r_{2}$ & $\Delta \mathrm{r}_{2}$ & $\Delta r_{2}^{\prime}$ \\
\hline$\Delta r_{3}$ & $\Delta \mathrm{r}_{3}$ & $\Delta r^{\prime}{ }_{3}$ & $\Delta x^{\prime}{ }_{3}$ & $\Delta x_{3}$ \\
\hline$\Delta r^{\prime} 3$ & $\Delta x^{\prime}{ }_{3}$ & $\Delta r_{3}$ & $\Delta r_{3}$ & $\Delta T^{\prime}{ }_{3}$ \\
\hline$\Delta \alpha_{1}$ & $\Delta \alpha_{1}$ & $\Delta \alpha_{1}^{\prime}$ & $\Delta a_{1}^{\prime}$ & $\Delta a_{1}$ \\
\hline$\Delta a_{2}$ & $\Delta \alpha_{2}$ & $\Delta x_{2}^{\prime}$ & $\Delta \Delta_{2}^{\prime}$ & $\Delta \alpha_{2}$ \\
\hline$\Delta \alpha_{3}$ & $\Delta a_{3}$ & $\Delta x^{\prime}{ }_{3}$ & $\Delta \alpha^{\prime}{ }_{3}$ & $\Delta \alpha_{3}$ \\
\hline$\Delta a_{1}$ & $\Delta a_{1}^{\prime}$ & $\Delta \alpha_{1}$ & $\Delta \alpha_{1}$ & $\Delta \alpha_{1}^{\prime}$ \\
\hline$\Delta a_{2}^{\prime}$ & $\Delta a_{2}^{\prime}$ & $\Delta a_{2}$ & $\Delta a_{2}$ & $\Delta \alpha_{2}^{\prime}$ \\
\hline$\Delta \alpha^{2}{ }_{3}$ & $\Delta \alpha_{3}$ & $\Delta a_{3}$ & $\Delta a_{3}$ & $\Delta a_{3}^{\prime}$ \\
\hline$\Delta B$ & $\Delta E$ & $\Delta \beta^{\prime}$ & $\Delta B^{\circ}$ & $\Delta 6$ \\
\hline$\Delta \beta^{\prime}$ & $\Delta B^{\prime}$ & $\Delta B$ & $\Delta B$ & $\Delta B^{\prime}$ \\
\hline
\end{tabular}


The generating co-ordinates for oxalic acid nay be written

as

$$
\text { Ag set } \begin{aligned}
S_{1} & =\Delta R \\
S_{2} & =\Delta r_{1}+\Delta r_{1}^{\prime} \\
S_{3} & =\Delta r_{2}+\Delta r_{2}^{\prime} \\
S_{4} & =\Delta r_{3}+\Delta r_{3}^{\prime} \\
S_{5} & =\left(\Delta a_{3}+\Delta a_{3}^{\prime}\right)-\left(\Delta a_{1}+\Delta a_{1}^{\prime}+\Delta a_{2}+\Delta a_{2}^{\prime}\right) \\
S_{6} & =\left(\Delta a_{2}+\Delta a_{2}^{\prime}\right)-\left(\Delta a_{1}+\Delta a_{1}^{\prime}\right) \\
S_{7} & =\Delta a_{3}+\Delta a_{1}+\Delta a_{2}+\Delta a_{3}^{\prime}+\Delta \alpha_{1}^{\prime}+\Delta \alpha_{2}^{\prime} \\
S_{8} & =\Delta \beta_{3}+\Delta \beta^{\prime}
\end{aligned}
$$

Bu sot $S_{9}=\Delta r_{1}-\Delta r_{1}^{\prime}$

$$
\begin{aligned}
& S_{10}=\Delta r_{2}-\Delta r_{2}^{\prime} \\
& S_{11}=\Delta r_{3}-\Delta r_{3}^{\prime} \\
& S_{12}=\left(\Delta a_{3}-\Delta \alpha_{3}^{\prime}\right)-\left(\Delta a_{2}-\Delta \alpha_{2}^{\prime}+\Delta \alpha_{1}-\Delta a_{1}^{\prime}\right) \\
& S_{13}=\left(\Delta \alpha_{2}-\Delta \alpha_{2}^{\prime}-\Delta \alpha_{1}+\Delta \alpha_{1}^{\prime}\right) \\
& S_{14}=\left(\Delta \alpha_{3}+\Delta \alpha_{1}+\Delta a_{2}\right)-\left(\Delta a_{3}^{\prime}+\Delta \alpha_{1}^{\prime}+\Delta a_{2}^{\prime}\right) \\
& S_{15}=\Delta \beta-\Delta \beta^{\prime}
\end{aligned}
$$

$S_{7}$ and $S_{14}$ can be picked as redundant co-ordinates because the sum of all angle changes around a point in a plane would be zero and thus not affect the kinetic or potential energies. So the generating co-ordinates reduce from 15 to 13 which correspond to the 13 inplane vibrations of the gaseous oxalic acid molecule. Normalized symetry co-ordinates may be obtained using equation 3.12 with Table 12 and nomalized using equation 3.13 . 
Thus, the nomalized symstry co-ordinates of gaseous oxalic acid 1st rodel may be written as

Ag set

$$
\begin{aligned}
& S_{1}=\Delta R \quad \text { symmotric C - C stretch } \\
& S_{2}=\frac{1}{\sqrt{2}}\left(\Delta x_{1}+\Delta r_{1}{ }_{1}\right) \quad " \quad C=0 \quad " \\
& S_{3}=\frac{1}{\sqrt{2}}\left(\Delta r_{2}+\Delta r^{\prime}{ }_{2}\right) \quad \text { " } c-0 \text { " } \\
& s_{4}=\frac{1}{\sqrt{2}}\left(\Delta r_{3}+\Delta r_{3}^{2}\right) \quad " \quad 0-11 \quad \text { " } \\
& S_{5}-\frac{1}{\sqrt{3}}\left(\Delta a_{3}+\Delta a_{3}^{\prime}\right)-\frac{1}{2 \sqrt{3}}\left(\Delta a_{2}+\Delta a_{2}^{\prime}+\Delta a_{1}+\Delta a_{1}^{\prime}\right) \\
& \text { symatric }(-c \leqslant 0) \text { angle defomation } \\
& s_{6}=\frac{1}{2}\left(\Delta a_{2}-\Delta a_{1}+\Delta a_{2}^{\prime}-\Delta a_{1}^{\prime}\right) \\
& \text { symetric }(-\mathrm{C} \leqslant 9) \text { angle rocking } \\
& S_{7}=\frac{1}{\sqrt{2}}\left(\Delta B+\Delta Q^{\prime}\right) \text { symetric } C-0-1 \text { angle bending }
\end{aligned}
$$

Bu set

$$
\begin{aligned}
& S_{8}=\frac{1}{\sqrt{2}}\left(\Delta r_{1}-\Delta r_{1}^{\prime}\right) \text { anti-symmetric CoO stretch } \\
& s_{9}=\frac{1}{\sqrt{2}}\left(\Delta r_{2}-\Delta r_{2}^{\prime}\right) \quad " \quad C-0 \quad " \\
& S_{10}=\frac{1}{\sqrt{2}}\left(\Delta r_{3}-\Delta r_{3}^{\prime}\right) \quad \because \quad 0-4 \quad " \\
& S_{11}-\frac{1}{\sqrt{3}}\left(\Delta a_{3}-\Delta \alpha_{3}^{\prime}\right)-\frac{1}{2 \sqrt{3}}\left(\Delta \alpha_{2}-\Delta \alpha_{2}^{\prime}+\Delta \alpha_{1}-\Delta \alpha_{1}^{\prime}\right) \\
& \text { anti-symetric }(-C \leqslant 0) \text { angle deformation } \\
& S_{12}=\frac{1}{2}\left(\Delta a_{2}-\Delta a_{2}^{\prime}-\Delta a_{1}+\Delta a_{1}^{\prime}\right) \\
& \text { anti-symetric }(-c \leqslant \rho) \text { angle rocking } \\
& S_{13}=\frac{1}{\sqrt{2}}\left(\Delta \beta-\Delta \beta^{\prime}\right) \quad \text { anti-symmetric } c-0-41 \text { angle bending }
\end{aligned}
$$

From the nornalized sywatry co-ordinates, the U matrix may be obtained as shom in Table 13. 
Table 15

U-matrix for gascous oxalic acid 1st model

\begin{tabular}{|c|c|c|c|c|c|c|c|c|c|c|c|c|c|c|}
\hline$s_{1}$ & 1 & & & & & & & & & & & & & \\
\hline $\mathbf{s}_{2}$ & & $\frac{3}{\sqrt{2}}$ & $\frac{1}{\sqrt{2}}$ & & & & & & & & & & & \\
\hline$s_{3}$ & & & & $\frac{1}{\sqrt{2}}$ & $\frac{1}{\sqrt{2}}$ & & & & & & & & & \\
\hline$s_{4}$ & & & & & & $\frac{1}{\sqrt{2}}$ & $\frac{1}{\sqrt{2}}$ & & & & & & & \\
\hline$s_{5}$ & & & & & & & & $-2 \frac{1}{\sqrt{3}}$ & $-2 \frac{1}{\sqrt{3}}$ & $\frac{1}{\sqrt{3}}$ & $-2 \frac{1}{2 \sqrt{3}}$ & $-\frac{1}{2 \sqrt{3}}$ & $\frac{1}{\sqrt{3}}$ & \\
\hline$s_{6}$ & & & & & & & & -1 & $\frac{1}{2}$ & & -1 & 1 & & \\
\hline$s_{7}$ & & & & & & & & & & & & & & 1 \\
\hline$s_{8}$ & & $\frac{1}{\sqrt{2}}$ & $\frac{1}{\sqrt{2}}$ & & & & & & & & & & & \\
\hline 9 & & & & $\frac{1}{\sqrt{2}}$ & $-\frac{1}{\sqrt{2}}$ & & & & & & & & & \\
\hline 10 & & & & & & $\frac{1}{\sqrt{2}}$ & $-\frac{1}{\sqrt{2}}$ & & & & & & & \\
\hline$s_{11}$ & & & & & & & & $-2 \frac{1}{73}$ & $-2 \frac{1}{27}$ & $\frac{1}{\sqrt{3}}$ & $\begin{array}{c}1 \\
273 \\
\end{array}$ & $\frac{1}{2 \sqrt{3}}$ & $\begin{array}{l}-1 \\
\sqrt{3} \\
\end{array}$ & \\
\hline$s_{12}$ & & & & & & & & -1 & $\frac{1}{2}$ & & $\frac{1}{2}$ & $-\frac{1}{2}$ & & \\
\hline 13 & & & & & & & & & & & & & & $\sqrt{2}$ \\
\hline
\end{tabular}


15.5 Numerical values of G-matrix in symatry co-ordinates

$$
\begin{aligned}
& G(1,1) \quad 0.166667 \\
& \mathrm{G}(1,2)=-0.064359 \\
& G(1,3)=-0.043957 \\
& G(1,4)=0 \\
& G(1,5) \quad 0.254615 \\
& G(1,6)=-0.00751 \\
& \mathrm{G}(1,7)=-0.097107 \\
& G(1,8) \quad=G(1,9)=G(1,10)=G(1,11)=G(1,12)=G(1,13)=0 \\
& G(2,1) \quad=-0.064359 \\
& G(2,2)=0.145833 \\
& G(2,3)=-0.047798 \\
& G(2,4)=0 \\
& G(2,5)=-0.035632 \\
& G(2,6)=0.210703 \\
& G(2,7)=0.060621 \\
& G(2,8) \quad-G(2,9)-G(2,10)-G(2,11)-G(2,12)=G(2,13)=0 \\
& G(3,1) \quad--0.043957 \\
& G(3,2)=-0.047798 \\
& G(3,3)=0.145835 \\
& G(3,4) \quad=-0.015543 \\
& G(3,5)=-0.081206 \\
& G(3,6) \quad-0.228183 \\
& G(3,7)=-0.058167 \\
& G(3,8)=G(3,9)=G(3,10)=G(3,11)=G(3,12)=G(3,13)=0
\end{aligned}
$$




$$
\begin{aligned}
& G(4,1)=0 \\
& G(4,2)=0 \\
& G(4,3)=-0.015543 \\
& G(4,4)=1.052491 \\
& G(4,5)=0.071662 \\
& G(4,6)=-0.042806 \\
& G(4,7)=-0.05376 \\
& G(4,8)=G(4,9)=G(4,10)=G(4,11)=G(4,12)=G(4,13)=0 \\
& G(5,1)=0.254615 \\
& G(5,2)=-0.085632 \\
& G(5,3)=-0.081206 \\
& G(5,4)=0.071662 \\
& G(5,5)=0.579780 \\
& G(5,6)=0.037330 \\
& G(5,7)=-0.229755 \\
& G(5,8)=G(5,9)=G(5,10)=G(5,11)=G(5,12)=G(5,13)=0 \\
& G(6,8)=0(6,9)=G(6,10)=G(6,11)=G(6,12)=G(6,13)=0 \\
& G(6,1)=-0.00751 \\
& G(6,2)=0.210708 \\
& G(6,3)=-0.228183 \\
& G(6,4)=-0.042806 \\
& G(6,5)=0.03733 \\
& G(6,6)=0.801268 \\
& G(6,7)=0.138167 \\
& G=0
\end{aligned}
$$




$$
\begin{aligned}
& G(7,1)=-0.097107 \\
& G(7,2) \quad-0.060521 \\
& G(7,3)=-0.058167 \\
& G(7,4) \quad-0.05376 \\
& G(7,5) \quad-0.229755 \\
& G(7,6) \quad-0.138167 \\
& G(7,7)=1.493328 \\
& G(7,8) \quad-G(7,9)=G(7,10)=G(7,11)-G(7,12)=G(7,13)=0 \\
& G(8,1)=G(8,2)=G(8,3)=G(8,4)=G(8,5)=G(8,6)-G(8,7)=0 \\
& \mathrm{G}(8,8)=0.145833 \\
& G(8,9)=-0.047798 \\
& G(8,10)=0 \\
& G(8,11)=-0.080808 \\
& G(8,12)=0.048269 \\
& G(8,13)=0.060621 \\
& G(9,1)=G(9,2)=G(9,3)=G(9,4)=G(9,5)=G(9,6)=G(9,7)=0 \\
& \mathrm{G}(9,8)=-0.047798 \\
& G(9,9)=0.145835 \\
& G(9,10)=-0.015545 \\
& G(9,11)=-0.086549 \\
& G(9,12)=-0.048269 \\
& G(9,13)=-0.068167
\end{aligned}
$$




$$
\begin{aligned}
& \mathrm{G}(10,1)=\mathrm{G}(10,2)=\mathrm{G}(10,3)=\mathrm{G}(10,4)=\mathrm{G}(10,5)=\mathrm{G}(10,6) \\
& -6(10,7)=0 \\
& G(10,8)=0 \\
& G(10,9)=-0.015543 \\
& \mathrm{G}(10,10)=1.062491 \\
& G(10,11) \quad 0.071662 \\
& \mathrm{G}(10,12)=-0.042806 \\
& G(10,13)=-0.05376 \\
& G(11,1)=G(11,2)=G(11,3)=G(11,4)-G(11,5)=G(11,6) \\
& =6(11,7)=0 \\
& 6(11,8)=-0.080808 \\
& \mathrm{G}(11,9)=-0.086549 \\
& G(11,10)=0.071662 \\
& \mathrm{G}(11,11)=0.58227 \\
& G(11,12)=0.005829 \\
& \mathrm{G}(11,13)=-0.227848 \\
& G(12,1)=G(12,2)=G(12,3)=G(12,4)=G(12,5)=G(12,6) \\
& =6(12,7) \quad 0 \\
& G(12,8)=0.048269 \\
& \mathrm{G}(12,9)=-0.048269 \\
& G(12,10) \quad-0.042806 \\
& G(12,11)=0.005829 \\
& \mathrm{G}(12,12)=0.098036 \\
& G(12,13)=0.073938
\end{aligned}
$$




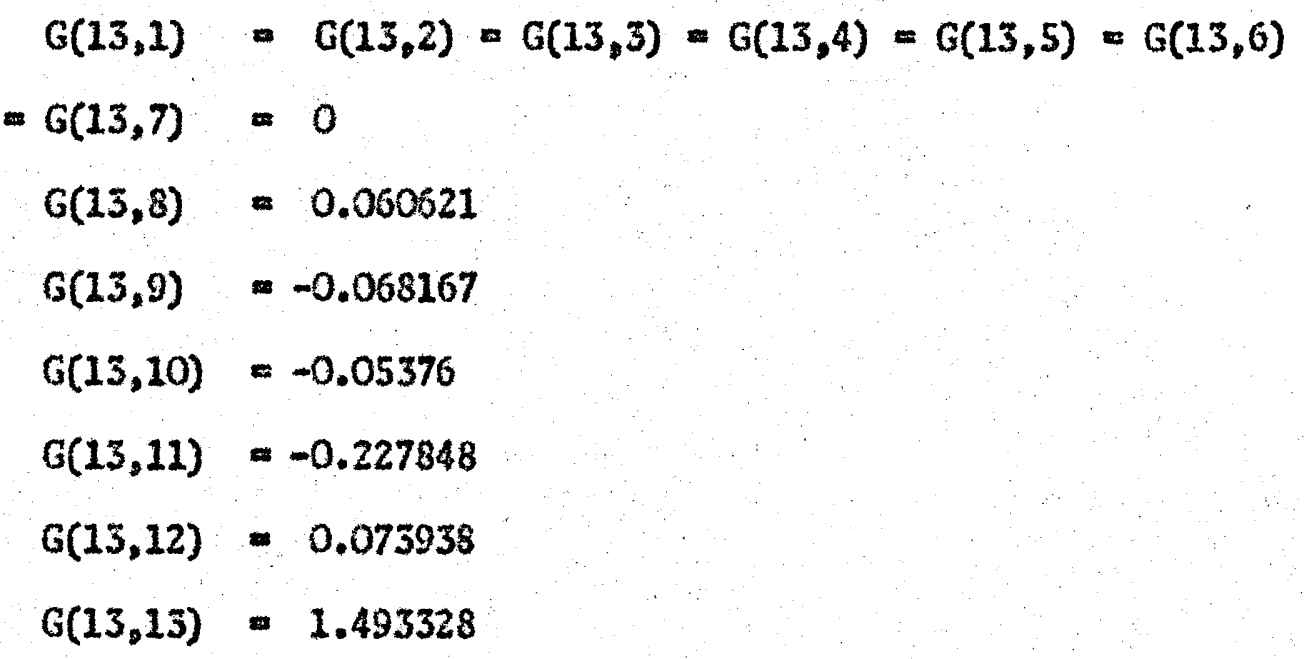




\section{CAAPTER 16}

CONSTRUCTION OF THE F-MATRIX OF THE INPLANE VIBRATIONS

OF GASEOUS OXALIC ACID 1ST MDDEL

16.1 Uroy-Brad1ey force field of gaseous oxalic acid (1st model)

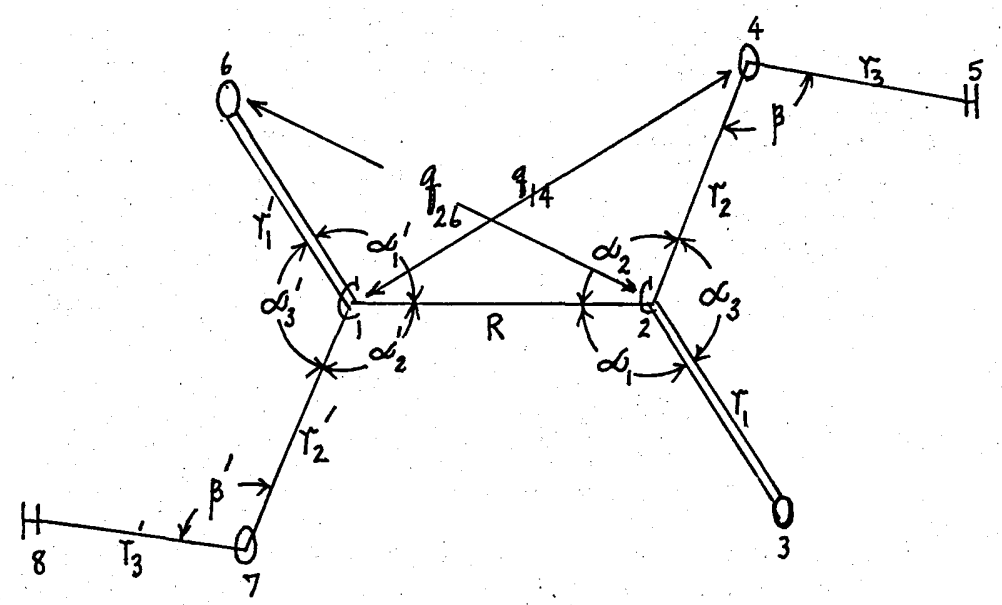

Fig. 20

The potential energy of gaseous oxalic acid can be expressed as

$$
\begin{aligned}
& V={ }^{1} K_{C C}(\Delta R)^{2}+K_{C C}^{R(\Delta R)} \\
& +1 \mathrm{~K}_{\mathrm{C}=0}\left(\Delta \mathrm{r}_{1}\right)^{2}+\mathrm{K}_{\mathrm{C}=0}^{1} \mathrm{r}_{1}\left(\Delta \mathrm{r}_{1}\right) \\
& +1 \mathrm{~K}_{\mathrm{C}=0}\left(\Delta \mathrm{r}_{1}^{\prime}\right)^{2}+\mathrm{K}_{\mathrm{C}=0}^{\prime} \mathrm{r}_{1}{ }_{1}\left(\Delta \mathrm{r}_{1}^{\prime}\right) \\
& +\frac{1}{2} K_{C-0}\left(\Delta x_{2}\right)^{2}+K_{C-O}^{1} r_{2}\left(\Delta x_{2}\right) \\
& +{ }_{2}^{1} K_{C-0}\left(\Delta r_{2}^{\prime}\right)^{2}+K_{C}^{\prime}-0^{r^{\prime}}{ }_{2}\left(\Delta r_{2}^{\prime}\right) \\
& +\frac{1}{2} K_{0-11}\left(\Delta r_{3}\right)^{2}+K_{0-H_{1}}^{1} r_{3}\left(\Delta r_{3}\right) \\
& +\frac{1}{2} K_{0-H 1}\left(\Delta r_{3}^{\prime}\right)^{2}+K_{0-H 1}^{\prime} r_{3}^{\prime}\left(\Delta r_{3}^{\prime}\right) \\
& +\frac{1}{2} H_{C-C \cos 0}\left(r_{1} \Delta \alpha_{1}\right)^{2}+H_{C-C=0} r_{1}\left(r_{1} \Delta a_{1}\right)
\end{aligned}
$$




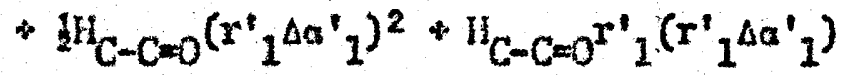

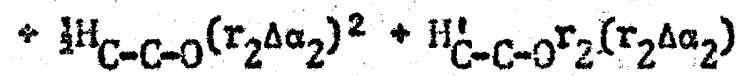

$$
\begin{aligned}
& +{ }_{2}^{1 H}{ }_{C-C-O}\left(r^{\prime}{ }_{2}^{\Delta \alpha^{\prime}{ }_{2}}\right)^{2}+H_{C}{ }^{C}-C-O^{r^{\prime}}{ }_{2}\left(r^{\prime}{ }_{2}^{\left.\Delta \alpha^{\prime}{ }_{2}\right)}\right. \\
& +1 H_{0-C \infty 0}\left(\sqrt{r_{1} r_{2}} \Delta a_{3}\right)^{2}+H_{-C=0} \sqrt{r_{1} r_{2}}\left(\sqrt{r_{1} r_{2}} \Delta a_{3}\right) \\
& +{ }_{2}^{1} H_{0-C=0}\left(\sqrt{r^{\prime}{ }_{1} r^{\prime}}{ }_{2} \Delta a^{\prime}{ }_{3}\right)^{2}+H_{0-C a O}\left(\sqrt{r^{\prime}{ }_{1} r^{\prime}} 2^{\Delta a^{\prime}}\right) \sqrt{r_{1}^{\prime} r_{2}^{\prime}}
\end{aligned}
$$

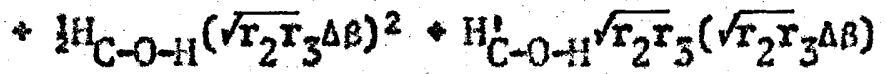

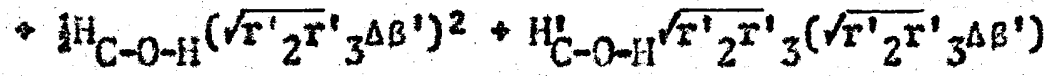

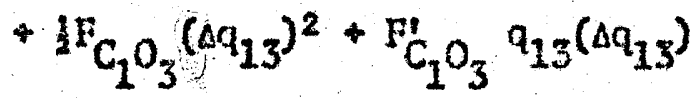

$$
\begin{aligned}
& +\frac{1}{2} \mathrm{P}_{2} \mathrm{O}_{6}\left(\Delta q_{26}\right)^{2}+\mathrm{F}_{\mathrm{C}_{2} \mathrm{O}_{6}} \mathrm{q}_{26}\left(\Delta q_{26}\right)
\end{aligned}
$$

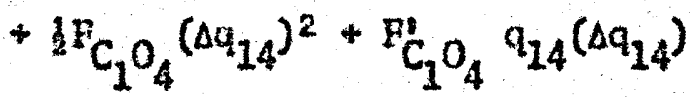

$$
\begin{aligned}
& +\frac{1}{2} \mathrm{~F}_{\mathrm{C}_{2} \mathrm{O}_{7}}\left(\Delta \mathrm{q}_{27}\right)^{2}+\mathrm{F}_{\mathrm{C}_{2} \mathrm{O}_{7} \mathrm{q}_{27}\left(\Delta \mathrm{q}_{27}\right)} \\
& +\frac{1}{2} \mathrm{~F}_{3} \mathrm{O}_{4}\left(\Delta q_{34}\right)^{2}+\mathrm{p}_{\mathrm{O}_{3} \mathrm{O}_{4}} \mathrm{a}_{34}\left(\Delta q_{34}\right) \\
& +\frac{1}{2} \mathrm{~F}_{6} \mathrm{O}_{7}\left(\Delta q_{67}\right)^{2}+\mathrm{F}_{6} \mathrm{O}_{7} q_{67}\left(\Delta q_{67}\right)
\end{aligned}
$$

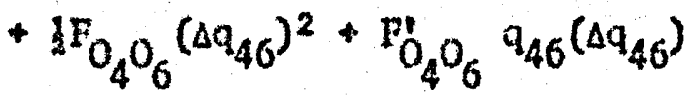

$$
\begin{aligned}
& +1 \mathrm{~F}_{\mathrm{O}_{3} \mathrm{O}_{7}}\left(\Delta q_{37}\right)^{2}+\mathrm{F}_{5} \mathrm{O}_{7} \mathrm{q}_{37}\left(\Delta q_{37}\right) \\
& +\frac{1}{2} \mathrm{C}_{2} \mathrm{H}_{5}\left(\Delta q_{25}\right)^{2}+\mathrm{F}_{\mathrm{C}_{2}} \mathrm{H}_{5} q_{25}\left(\Delta q_{25}\right) \\
& +1 \mathrm{~F}_{\mathrm{C}_{1} \mathrm{H}_{8}}\left(\Delta \mathrm{q}_{18}\right)^{2}+\mathrm{F}_{\mathrm{C}_{1} \mathrm{H}_{8}} \mathrm{q}_{18}\left(\Delta \mathrm{q}_{18}\right)
\end{aligned}
$$

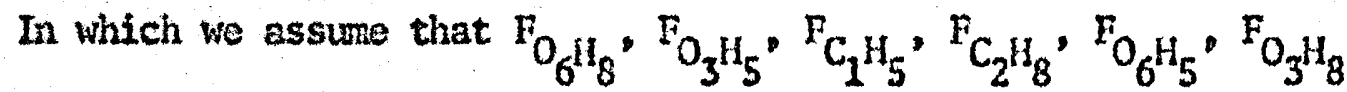
and $\mathrm{F}_{5} \mathrm{H}_{8}$ are small compared with each of the force constants above and we neglect them to make the calculation possible. 
For simplicity of writing let

$$
\begin{aligned}
& K_{C C}=K_{1} \\
& K_{C \infty O}=K_{2} \\
& K_{C-O}=K_{3} \\
& K_{O-H}=K_{4} \\
& H_{C-C \infty O}=H_{1} \\
& H_{C-C-O}=H_{2} \\
& H_{O-C=O}=H_{3} \\
& H_{C-O-H}=H_{4} \\
& F_{C_{1} O_{3}}=F_{C_{2} O_{6}}=F_{1} \\
& F_{C_{1} O_{4}}=F_{C_{2} O_{7}}=F_{2} \\
& F_{O_{3} O_{4}}=F_{O_{6} O_{7}}=F_{3} \\
& F_{O_{4} O_{6}}=F_{O_{3} O_{7}}=F_{4} \\
& F_{C_{2} H_{5}}=F_{C_{1} H_{8}}=F_{5}
\end{aligned}
$$

So there are 13 force constants in the Urey-Bradiey force field of the inplane vibration of the gaseous oxalic acid molecule.

\subsection{Evaluation of F-natrix}

The first step in the evaluation of the F-matrix is to eliminate all $\Delta q$ in the potential enorgy expression. Using Table 1 and 2 in section 6.3 and substituting all the $q$ values in the Urey-Bradley potential energy of gaseous oxalic acid molecule and neglecting all first ordor tems, the potential expression in 
$-137-$

torms of intemal co-ordinates of gaseous oxalic acid is

$$
\begin{aligned}
& V=\frac{1}{2}(\Delta R)^{2}\left(\mathrm{X}_{\mathrm{CC}}+\mathrm{s}^{2}{ }_{13} \mathrm{~F}_{1}+t^{2}{ }_{13} \mathrm{~F}_{1}^{\prime}+\mathrm{s}^{2}{ }_{26} \mathrm{~F}_{1}+t^{2}{ }_{26} \mathrm{~F}_{1}^{\prime}+\mathrm{s}^{2}{ }_{14} \mathrm{~F}_{2}\right. \\
& \left.+t_{14}^{2} F_{2}^{\prime}+s_{27}^{2} F_{2}+t_{27}^{2} F_{2}^{\prime}+a_{64}^{2} F_{4}+b_{64}^{2} F_{4}^{\prime \prime}+a_{37}^{2} F_{4}+b^{2}{ }_{37} F_{4}^{\prime}\right) \\
& +\frac{1}{2}\left(\Delta \mathrm{r}_{1}\right)^{2}\left(\mathrm{~K}_{C=0}+\mathrm{S}^{2}{ }_{31} \mathrm{~F}_{1}+t^{2}{ }_{31} \mathrm{~F}_{1}+\mathrm{s}_{34}^{2} \mathrm{~F}_{3}+t^{2}{ }_{34} \mathrm{~F}_{3}+v_{37}^{2} \mathrm{~F}_{4}\right. \\
& \left.+\left(1-v_{37}^{2}\right) F_{4}^{\prime}\right) \\
& +\frac{1}{2}\left(\Delta \mathrm{r}_{1}^{\prime}\right)^{2}\left(\mathrm{~K}_{\mathrm{C}=0}+\mathrm{s}_{62}^{2} \mathrm{~F}_{1}+t_{62}^{2} \mathrm{~F}_{1}+s_{67}^{2} \mathrm{~F}_{3}+t_{67}^{2} \mathrm{~F}_{3}^{\prime}+v_{64}^{2} \mathrm{~F}_{4}\right. \\
& \left.+\left(1-v^{2}{ }_{64}\right) F_{4}\right) \\
& +\frac{1}{2}\left(\Delta r_{2}\right)^{2}\left(K_{C-O}+s^{2}{ }_{41} F_{2}+t_{41}^{2} F_{2}^{\prime}+S^{2}{ }_{45} F_{3}+t^{2}{ }_{43} F_{3}^{\prime}+s^{2} 5_{5}\right. \\
& \left.+t^{2} 5^{F \prime}{ }_{5}+v^{2}{ }_{46} F_{4}+\left(1-v_{46}^{2}\right) H_{4}\right) \\
& +\frac{1}{2}\left(\Delta r_{2}^{\prime}\right)^{2}\left(K_{C-0}+s^{2}{ }_{72} F_{2}+t^{2}{ }_{72} F_{2}^{\prime}+s^{2}{ }_{76} F_{3}+t^{2}{ }_{76} F_{3}^{\prime}+s^{2} F_{5}\right. \\
& \left.+t^{2}{ }_{18} F_{5}^{\prime}+V_{73}^{2} F_{4}+\left(1-v_{73}^{2}\right) F_{4}^{\prime}\right) \\
& +1\left(\Delta \mathrm{r}_{3}\right)^{2}\left(\mathrm{~K}_{\mathrm{O}-\mathrm{H}}+\mathrm{s}_{52}^{2} \mathrm{~F}_{5}+\mathrm{t}^{2}{ }_{52} \mathrm{~F}_{5}\right) \\
& +1\left(\Delta t^{\prime}{ }_{3}\right)^{2}\left(K_{O-H}+s^{2} F_{5} F_{5}+t^{2}{ }_{81} F^{\prime}{ }_{5}\right) \\
& +\frac{1}{2}\left(\mathrm{r}_{1} \Delta \mathrm{e}_{1}\right)^{2}\left(\mathrm{H}_{\mathrm{C}-\mathrm{CoO} O}+\frac{\mathrm{R}}{\mathrm{r}_{1}}\left(t_{13} \mathrm{t}_{31} \mathrm{~F}_{1}-\mathrm{S}_{13} \mathrm{~S}_{31} \mathrm{~F}_{1}\right)+\mathrm{u}_{37}^{2} \mathrm{~F}_{4}+\left(\mathrm{v}_{37} \mathrm{q}_{37}\right)\right. \\
& \left.\left.\left.r_{1}\right)+\left(1-u^{2}, 3\right)\right\} F^{\prime}\right)
\end{aligned}
$$

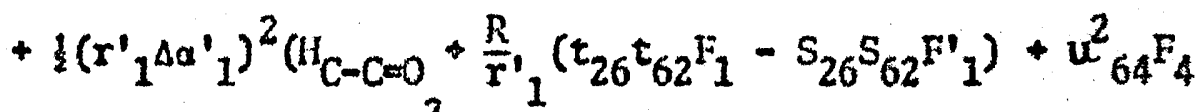

$$
\begin{aligned}
& +\left(\left(v_{64} q_{64} / r_{1}\right)+\left(1-u_{64}^{2}\right) r^{\prime}{ }_{4}\right) \\
& +\frac{1}{2}\left(r_{2} \Delta \alpha_{2}\right)^{2}\left(H_{C-C-O}+\frac{R}{r_{2}}\left(t_{14} t_{41} F_{2}-S_{14} S_{41} F_{2}^{\prime}\right)+u_{46}^{2} F_{4}\right. \\
& \left.+\left(\left(v_{46} q_{64} / r_{2}\right)+\left(1-u_{46}^{2}\right)^{2}\right) r^{\prime}\right)
\end{aligned}
$$




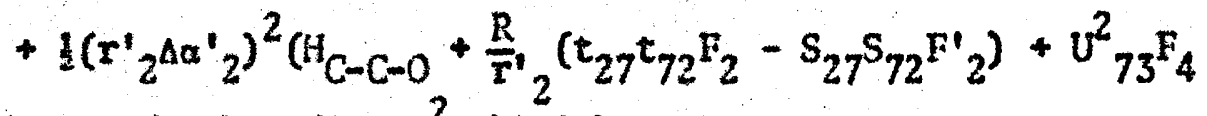

$$
\begin{aligned}
& \left.+\left(\left(v_{73} q_{37} / r_{2}^{\prime}\right)+\left(1-U_{73}^{2}\right)\right\} F_{4}^{\prime}\right) \\
& +\frac{1}{3}\left(\sqrt{r_{1} x_{2} \Delta \alpha_{3}}\right)^{2}\left(H_{0-C \infty O}+t_{34} t_{43} F_{3}-s_{34} S_{43} F_{3}\right) \\
& \text { + } 1\left(\sqrt{r^{\prime}{ }_{1}{ }^{\prime}}{ }_{2} \alpha^{\prime}{ }_{3}\right)^{2}\left(\mathrm{H}_{0}-\mathrm{C}=0+t_{67} t_{76} F_{3}-S_{67} S_{76} F_{3}\right) \\
& +\frac{1}{2}\left(\sqrt{r_{2} r_{3}} 3^{2 B}\right)^{2}\left(I_{C-O-1 I}+t_{25} t_{52} F_{5}-S_{25} S_{52} F^{\prime}\right)
\end{aligned}
$$

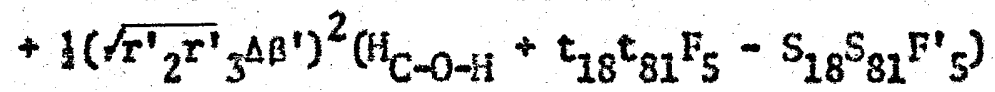

$$
\begin{aligned}
& +(\Delta R)\left(\Delta r_{1}\right)\left(S_{13} S_{31} F_{1}-\tau_{13} t_{31} F_{1}^{\prime}-a_{37} v_{37} F_{4}+\left(a_{37} v_{37}-\cos a_{1}\right) F_{4}^{\prime}\right) \\
& +(\Delta R)\left(\Delta r_{1}^{\prime}\right)\left(S_{26} S_{62} F_{1}-t_{26} t_{62} F_{1}^{\prime}-a_{64} v_{64} F_{4}+\left(a_{64} v_{64}-\cos a_{1}^{\prime}\right) F_{4}^{\prime}\right) \\
& +(\Delta R)\left(\Delta r_{2}\right)\left(S_{14} S_{41} F_{2}-t_{14} t_{41} F_{2}^{\prime}-a_{64} v_{46} F_{4}+\left(a_{64} v_{46}-\cos \alpha_{2}\right) F_{4}^{\prime}\right) \\
& +(\Delta B)\left(\Delta r_{2}^{\prime}\right)\left(S_{27} S_{72} F_{2}-t_{27} t_{72} F_{2}^{\prime}-a_{37} v_{73} F_{4}+\left(a_{37} v_{73}-\cos a_{2}^{\prime}\right) F_{4}^{\prime}\right) \\
& +\Delta R\left(r_{1} \Delta a_{1}\right) \sqrt{\frac{R}{r_{1}} S_{13}} \sqrt{t_{13} t_{31}} F_{1}+t_{13} S_{31} F_{1}^{\prime}+v_{37} a_{37} F_{4} \\
& \left.+\left(\sin \alpha_{1}-a_{37} U_{37}\right){ }^{\prime}{ }_{4}\right)
\end{aligned}
$$

$$
\begin{aligned}
& 4 \Delta R\left(r_{1}^{\prime} \Delta \alpha_{1}^{\prime}\right)\left(\sqrt{\frac{R}{r^{\prime}}} S_{1} S_{26} \sqrt{t_{26} t_{62} F_{1}}+t_{26} S_{62} F_{1}^{\prime}+U_{64} a_{04} F_{4}\right. \\
& \left.+\left(\sin \alpha_{1}^{\prime}-a_{64} u_{64}\right) F^{\prime}{ }_{4}\right) \\
& +(A R)\left(r_{2} \Delta a_{2}\right)\left(\sqrt{\frac{R}{r_{2}}} s_{14} \sqrt{t_{14} t_{41}} F_{2}+t_{14} S_{41} F_{2}^{\prime}+u_{46} a_{64} F_{4}\right. \\
& \left.+\left(\sin \alpha_{2}-a_{64} \mathrm{ta}_{46}\right) \mathrm{F}_{4}^{\prime}\right) \\
& +(\Delta D)\left(r_{2}^{\prime}{ }_{2} \Delta a_{2}^{\prime}\right)\left(\sqrt{\frac{R}{r^{\prime}}}{ }_{2} s_{27} \sqrt{t_{27} t_{72}} F_{2}+t_{27} S_{72} F_{2}^{\prime}+u_{73} a_{37} F_{4}\right. \\
& \left.+\left(\sin \alpha_{2}^{\prime}-a_{37}{ }^{\prime \prime}{ }_{73}\right) F_{4}^{\prime}\right) \\
& \begin{array}{l}
+\left(\Delta r_{1}\right)\left(\Delta r_{2}\right)\left(S_{34} S_{43} F_{3}-t_{34} t_{43} F_{3}^{\prime}\right) \\
+\left(\Delta r_{1}\right)\left(\Delta r_{2}^{\prime}\right)\left(v_{37} V_{73} F_{4}+\left(\cos \left(a_{2}^{\prime}+a_{1}\right)-v_{37} V_{73}\right) F_{4}^{\prime}\right)
\end{array}
\end{aligned}
$$


$+\left(\Delta r_{1}\right)\left(r_{1} \Delta \alpha_{2}\right)\left(\sqrt{\frac{R}{r_{1}}} s_{31} \sqrt{t_{13} t_{31}} F_{1}+\frac{R}{r_{1}} t_{31} s_{13} F_{1}^{E_{1}}-u_{37} v_{37} \mathrm{P}_{4}\right.$

$+\left\{u_{37}\left(q_{37}+r_{1} v_{37}\right) / r_{1}\right\} F_{4}^{\prime}$

$+\left(\Delta r_{1}\right)\left(\sqrt{r_{1} r_{2}} a_{3}\right)\left(s_{34} \sqrt{t_{34} \varepsilon_{43}} F_{3}+\sqrt{\frac{r_{2}}{r_{1}}} t_{34} s_{43} F^{\prime}{ }_{3}\right)$

$+\left(\Delta r_{1}\right)\left(r_{2}^{\prime} \Delta a_{2}^{\prime}\right)\left(-u_{73} v_{37} F_{4}+\left\{v_{37} u_{73}-\sin \left(a_{2}^{\prime}+a_{1}\right)\right\} F_{4}^{\prime}\right)$

$+\left(\Delta r_{1}^{\prime}\right)\left(\Delta r_{2}\right)\left(v_{64} v_{46} F_{4}+\left(\cos \left(a_{1}+a_{2}^{\prime}\right)-v_{64} v_{46}{ }^{\prime} F_{4}^{\prime}\right)\right.$

$+\left(\Delta \mathrm{r}_{1}{ }_{1}\right)\left(\Delta \mathrm{r}_{2}^{\prime}\right)\left(\mathrm{S}_{67} \mathrm{~S}_{76} \mathrm{~F}_{3}-\mathrm{t}_{67} \mathrm{t}_{76} \mathrm{~F}_{3}{ }_{3}\right)$

$+\left(\Delta r_{1}^{\prime}\right)\left(r_{2} \Delta a_{2}\right)\left(-u_{46} v_{64} F_{4}+\left\{v_{64} u_{46}-\sin \left(\alpha_{2}+\alpha_{1}^{\prime}\right)\right\} F_{4}^{\prime}\right)$

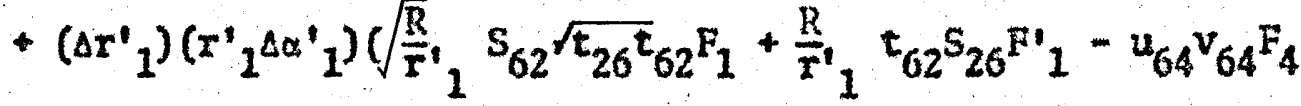

$\left.+\left\{u_{64}\left(\mathrm{q}_{64}+x^{\prime}{ }_{1} \mathrm{v}_{64}\right) / \mathrm{r}_{1}{ }_{1}\right\}^{\prime}{ }_{4}{ }_{4}\right)$

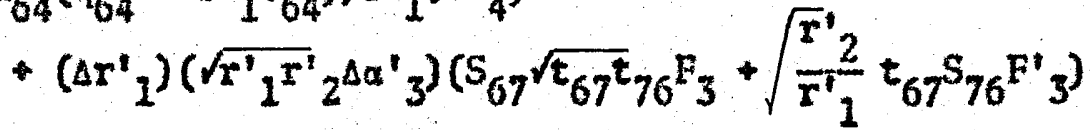

$+\left(\Delta \mathrm{r}_{2}\right)\left(\Delta \mathrm{r}_{3}\right)\left(\mathrm{S}_{25} \mathrm{~S}_{52} \mathrm{~F}_{5}-\mathrm{t}_{25} \mathrm{t}_{52} \mathrm{~F}_{5}{ }_{5}\right)$

$+\left(\Delta r_{2}\right)\left(r_{2} \Delta a_{2}\right)\left(\sqrt{\frac{R}{r_{2}}} s_{41} \sqrt{t_{14} t_{41}} F_{2}+\frac{R}{r_{2}} t_{41} s_{14} F_{2}^{\prime}-u_{46} v_{46} F_{4}\right.$

$\left.+\left(u_{46}\left(q_{64}+r_{2} v_{46}\right) / r_{2}\right\} F_{4}{ }_{4}\right)$

$+\left(\Delta r_{2}\right)\left(\sqrt{r_{1} r_{2}}{ }^{\Delta \alpha_{3}}\right)\left(s_{43} \sqrt{t_{34} t_{43}} F_{3}+\sqrt{\frac{r_{1}}{r_{2}}} t_{43} S_{34} F^{\prime}{ }_{3}\right)$

+ $\left(\Delta r_{2}\left(r_{1}^{\prime}{ }_{1} \Delta \alpha_{1}{ }_{1}\right)\left(-u_{64} v_{46} F_{4}+\left\{v_{46} u_{64}-\sin \left(a_{2}+\alpha_{1}^{\prime}{ }_{1}\right)\right\} F_{4}{ }_{4}\right)\right.$

$+\left(\Delta r_{2}\right)\left({ }_{r_{2}} r_{3} \Delta 3\right)\left(S_{25} \sqrt{t_{25} t_{52}} F_{5}+\sqrt{\frac{r_{3}}{r_{2}}} t_{25} S_{52} F_{5}^{\prime}\right)$

$+\left(\Delta \mathrm{r}_{2}{ }_{2}\right)\left(\Delta \mathrm{r}^{\prime}{ }_{3}\right)\left(\mathrm{S}_{18} \mathrm{~S}_{81} \mathrm{~F}_{5}-\mathrm{t}_{18} \mathrm{t}_{81} \mathrm{Fr}_{5}\right)$

+ $\left(\Delta r_{2}^{\prime}\right)\left(r_{1} \Delta a_{1}\right)\left(-u_{37} v_{73} F_{4}+\left(v_{73} u_{37}-\sin \left(a_{2}^{\prime}+a_{1}\right)\right] F_{4}^{\prime}\right)$

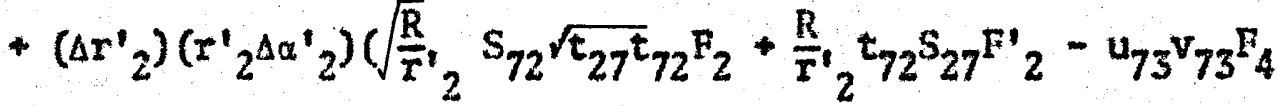

$\left.+\left(u_{73}\left(q_{37}+r^{\prime}{ }_{2}^{v} v_{73}\right) / x_{2}^{\prime}\right\}^{\prime} F_{4}^{\prime}\right)$

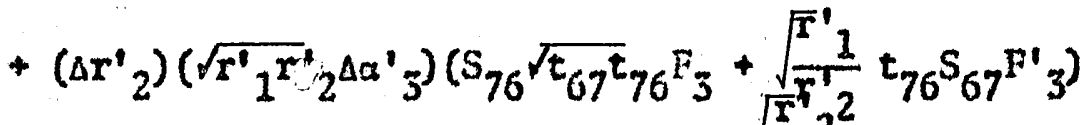

$+\left(\Delta r_{2}^{\prime}\right)\left(\sqrt{r^{\prime}{ }_{2}^{r^{\prime}}} 3^{\Delta \beta^{\prime}}\right)\left(S_{18} \sqrt{t_{18} t_{81}} F_{5}+\sqrt{\frac{r^{\prime}}{r^{\prime}{ }_{2}} t_{18}} t_{18} S_{81} F_{5}^{\prime}\right)$ 


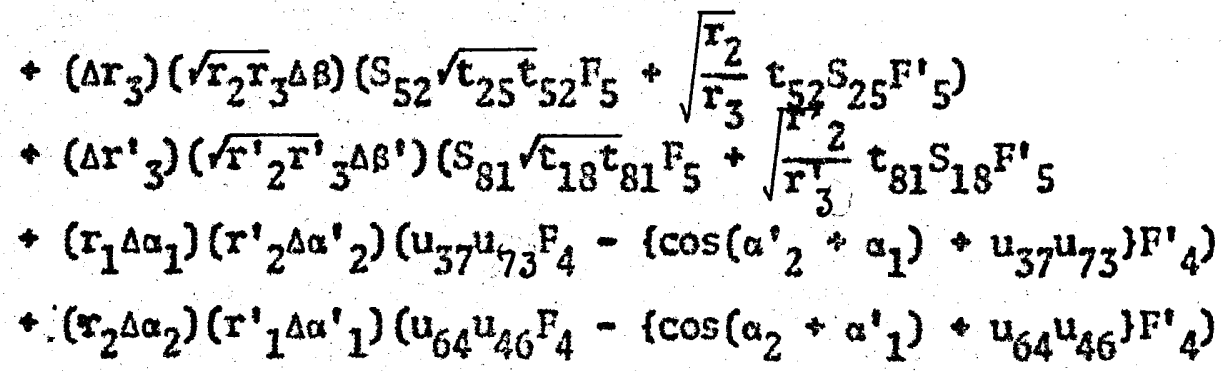

The numerical values in this expression are

$$
\begin{aligned}
& q_{13}=q_{26}=2.428573 \mathrm{~A}^{\circ} \\
& q_{14}=q_{27}=2.394888 \mathrm{~A}^{\circ} \\
& q_{25}=q_{18}=1.900354 \mathrm{~A}^{\circ} \\
& q_{34}=q_{67}=2.260029 \mathrm{~A}^{\circ} \\
& q_{64}=q_{37}=2.716907 \mathrm{~A}^{\circ} \\
& s_{13}=s_{26}=0.909048 \\
& s_{31}=s_{62}=0.845502 \\
& t_{13}=t_{26}=0.416692 \\
& t_{31}=t_{62}=0.533972 \\
& s_{14}=s_{27}=0.854918 \\
& s_{41}=s_{72}=0.80020 \\
& s_{14}=t_{27}=0.518762 \\
& t_{41}=t_{72}=0.599734 \\
& s_{34}=s_{67}=0.874336 \\
& s_{43}=s_{76}=0.899052 \\
& t_{34}=t_{67}=0.485322 \\
& t_{43}=t_{76}=0.437841
\end{aligned}
$$




$$
\begin{aligned}
& s_{25}=s_{18}=0.842799 \\
& s_{52}=s_{81}=0.730914 \\
& t_{25}=t_{18}=0.538226 \\
& t_{52}=t_{81}=0.682467
\end{aligned}
$$

$$
a_{64}=a_{57}=0.995399
$$$$
b_{64}=b_{37}=-0.084807
$$$$
u_{64}=u_{37}=0.881016
$$$$
u_{46}-u_{73}=0.392867
$$$$
v_{64}=v_{37}=-0.473089
$$$$
v_{46}=v_{73}=-0.450334
$$

The potential energy of gasoous oxalic acid (1st model) is given by substituting these values into the general expression and thus, only 13 force constants are left as follows:-

$$
\begin{aligned}
V & =\frac{1}{2}(\Delta R)^{2}\left(K_{C C}+1.51801 F_{1}+1.407947 F_{2}+1.984183 F_{4}\right) \\
& +\frac{1}{2}\left(\Delta r_{1}\right)^{2}\left(K_{C=0}+0.686361 F_{1}+0.74091 F_{3}+0.146195 F_{4}\right) \\
& +1\left(\Delta r_{1}^{\prime}\right)^{2}\left(K_{C=0}+0.686361 F_{1}+0.74091 F_{3}+0.146195 F_{4}\right) \\
& +1\left(\Delta r_{2}\right)^{2}\left(K_{C-0}+0.604352 F_{2}+0.789124 F_{3}+0.681341 F_{5}\right. \\
& \left.+0.123081 F_{4}\right) \\
& +1\left(\Delta r_{2}^{\prime}\right)^{2}\left(K_{C-0}+0.604352 F_{2}+0.739124 F_{3}+0.681341 F_{5}\right. \\
& \left.+0.123031 F_{4}\right) \\
& +\frac{1}{2}\left(\Delta r_{3}\right)^{2}\left(K_{0-11}+0.487659 F_{5}\right) \\
& +\frac{1}{2}\left(\Delta r_{3}{ }_{3}\right)^{2}\left(K_{0-H}+0.487659 F_{5}\right) \\
& +\frac{1}{2}\left(r_{1} \Delta \alpha_{1}\right)^{2}\left(I_{1}+0.33352 F_{1}+0.860210 F_{4}\right)
\end{aligned}
$$


- $\frac{1}{2}\left(r^{\prime}{ }_{2} \Delta \alpha_{1}{ }_{1}\right)^{2}\left(\mathrm{H}_{1}+0.38362 \mathrm{~F}_{1}+0.860210 \mathrm{~F}_{4}\right)$

$+1\left(\mathrm{r}_{2} \mathrm{Aa}_{2}\right)^{2}\left(\mathrm{H}_{2}+0.438769 \mathrm{~F}_{2}+0.368308 \mathrm{~F}_{4}\right)$

$+\frac{1}{2}\left(\mathrm{r}_{2}^{\prime}{ }_{2} \mathrm{a \alpha}_{2}{ }^{\prime}\right)^{2}\left(\mathrm{H}_{2}+0.438769 \mathrm{~F}_{2}+0.368308 \mathrm{~F}_{4}\right)$

$+1\left(\sqrt{r_{1} r_{2}} \Delta a_{3}\right)^{2}\left(H_{3}+0.291101 E_{3}\right)$

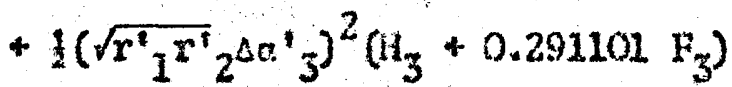

$+\frac{1}{2}\left(\sqrt{r_{2} r_{3}} \Delta \beta\right)^{2}\left(B_{4}+0.428923 F_{5}\right)$

$+1\left(\sqrt{r^{\prime}} 2^{r^{\prime}} 3^{\Delta \beta^{\prime}}\right)^{2}\left(\mathrm{H}_{4}+0.428923 \mathrm{~F}_{5}\right)$

- $(\Delta R)\left(\Delta r_{1}\right)\left(0.790352 E_{1}+0.463914 F_{4}\right)$

- $(\Delta R)\left(\Delta r_{1}^{\prime}\right)\left(0.790852 F_{1}+0.463914 F_{4}\right)$

$+(\Delta R)\left(\Delta r_{2}\right)\left(0.715217 F_{2}+0.456285 F_{4}\right)$

- $(A R)\left(\Delta r_{2}^{\prime}\right)\left(0.725217 F_{2}+0.456285 F_{4}\right)$

$+(A R)\left(r_{1} \Delta a_{1}\right)\left(0.450175 F_{1}+0.881856 F_{4}\right)$

$+(\Delta R)\left(x_{1}^{\prime} \Delta a_{1}^{\prime}\right)\left(0.450175 F_{1}+0.881 .856 F_{4}\right)$

- $(\Delta B)\left(\mathrm{r}_{2} \triangle \mathrm{CO}_{2}\right)\left(0.471212 \mathrm{~F}_{2}+0.885333 \mathrm{~F}_{4}\right)$

$+(\Delta \mathrm{R})\left(\mathrm{r}_{2}^{\prime}{ }_{2}^{\Delta \alpha^{\prime}{ }_{2}}\right)\left(0.471212 \mathrm{~F}_{2}+0.585833 \mathrm{~F}_{4}\right)$

$+\left(\Delta r_{1}\right)\left(\Delta r_{2}\right)\left(0.307323 F_{3}\right)$

$+\left(\Delta \mathrm{r}_{1}\right)\left(\Delta \mathrm{r}_{2}^{\prime}\right)\left(0.291711 E_{4}\right)$

$+\left(\Delta r_{1}\right)\left(r_{1} \Delta \alpha_{1}\right)\left(0.359272 F_{1}+0.260350 F_{4}\right)$

- $\left(\Delta r_{1}\right)\left({\sqrt{r_{1} r_{2}} \Delta \alpha_{3}}_{3}\left(0.357105 F_{3}\right)\right.$

$+\left(\Delta r_{1}\right)\left(r_{2}^{\prime} \Delta a_{2}^{\prime}\right)\left(0.382731 F_{4}\right)$

$+\left(\Delta \mathrm{r}_{1}^{\prime}\right)\left(\Delta \mathrm{r}_{2}\right)\left(0.291711 \mathrm{~F}_{4}\right)$

$+\left(\Delta r_{1}^{\prime}\right)\left(\Delta r_{2}^{\prime}\right)\left(0.607323 F_{3}\right)$

$+\left(\Delta r_{1}^{\prime}\right)\left(r_{2} \Delta \alpha_{2}\right)\left(0.382731 F_{4}\right)$

$+\left(\Delta r_{1}^{\prime}\right)\left(r^{\prime}{ }_{1}^{\Delta \alpha}{ }_{1}\right)\left(0.382272 F_{1}+0.260330 F_{4}\right)$

$+\left(\Delta r_{1}^{\prime}\right)\left(\sqrt{r^{\prime}{ }_{1} r^{\prime \prime}} 2^{\Delta \alpha^{\prime}}{ }_{3}\right)\left(0.357105 F_{3}\right)$

$+\left(\Delta r_{2}\right)\left(\Delta r_{3}\right)\left(0.652746 F_{5}\right)$ 
$+\left(\Delta \mathrm{r}_{2}\right)\left(\mathrm{r}_{2} \Delta \alpha_{2}\right)\left(0.420632 \mathrm{~F}_{2}+0.261129 \mathrm{~F}_{4}\right)$

$+\left(\Delta r_{2}\right)\left(\sqrt{r_{1} r_{2}} \Delta d_{3}\right)\left(0.378075 F_{3}\right)$

$+\left(\Delta r_{2}\right)\left(r_{1}^{\prime}{ }_{1} \Delta a_{1}^{*}\right)\left(0.354512 \mathrm{~F}_{4}\right)$

$+\left(\Delta r_{2}\right)\left(\sqrt{r_{2} r_{3}} \Delta B\right)\left(0.475860 \mathrm{~F}_{5}\right)$

$+\left(\Delta r_{2}^{\prime}\right)\left(\Delta x_{3}^{\prime}\right)\left(0.652746 F_{5}\right)$

$+\left(\Delta \mathrm{r}_{2}^{\prime}\right)\left(\mathrm{r}_{1} L \alpha_{1}\right)\left(0.354512 \mathrm{~F}_{4}\right)$

$+\left(\Delta x_{2}^{\prime}\right)\left(x^{\prime}{ }_{2} \Delta \alpha_{2}^{\prime}\right)\left(0.420632 F_{2}+0.261129 F_{4}\right)$

$+\left(\Delta \mathrm{r}_{2}^{\prime}\right)\left(\sqrt{\mathrm{x}^{\prime}{ }_{1} \mathrm{r}^{\prime}} 2^{\Delta c^{\prime}}{ }_{3}\right)\left(0.378075 \mathrm{~F}_{3}\right)$

$+\left(\Delta \mathrm{r}_{2}^{\prime}\right)\left(\sqrt{\mathrm{r}^{\prime}{ }_{2}^{\mathrm{r}^{\prime}}} 3^{\Delta \theta^{\prime}}\right)\left(0.475860 \mathrm{~F}_{5}\right)$

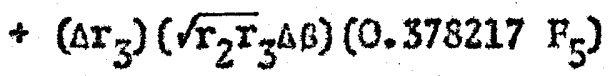

$+\left(\Delta r_{3}^{\prime}\right)\left(\sqrt{r^{\prime} 2^{r^{\prime}}} \Delta^{\Delta B^{\prime}}\right)\left(0.378217 \mathrm{~F}_{5}\right)$

$+\left(r_{1} \Delta c_{1}\right)\left(r^{\prime}{ }_{2} \Delta a^{\prime}{ }_{2}\right)\left(0.807935 F_{4}\right)$

$+\left(r_{2} \Delta \alpha_{2}\right)\left(r^{0}{ }_{1} \Delta \alpha^{\prime}{ }_{1}\right)\left(0.807935 F_{4}\right)$

16.3 F-matrix in intemal co-ordinates of gaseous oxalic acid (1st modo1)

$$
\begin{aligned}
& F(1,1)=K_{1}=1.61801 F_{1}+1.407947 F_{2}+1.984183 F_{4} \\
& F(1,2)=0.790852 F_{1}+0.463914 F_{4} \\
& F(1,3)=0.790852 F_{1}+0.463914 F_{4} \\
& F(1,4)=0.715217 F_{2}+0.456285 F_{4} \\
& F(1,5)=0.715217 F_{2}+0.456285 F_{4} \\
& F(1,3)=0.450175 F_{1}+0.881856 F_{4} \\
& F(1,9)=0.471212 F_{2}+0.885833 F_{4} \\
& F(1,11)=0.450175 F_{1}+0.881856 F_{4} \\
& F(1,12)=0.471212 F_{2}+0.885833 F_{4} \\
& F(1,0)=F(1,7)=F(1,10)=F(1,13)=F(1,14)=F(1,15)=0
\end{aligned}
$$




$$
\begin{aligned}
& \mathrm{F}(2,1) \quad=0.790852 \mathrm{~F}_{1}+0.463914 \mathrm{~F}_{4} \\
& F(2,2)=K_{2}+0.686361 F_{1}+0.74091 F_{3}+0.146195 F_{4} \\
& F(2,4)=0.807323 F_{3} \\
& F(2,5)=0.291711 \mathrm{~F}_{4} \\
& F(2,8)=0.389272 F_{1}+0.260330 F_{4} \\
& F(2,10)=0.357105 \mathrm{~F}_{3} \\
& F(2,12)=0.382731 \mathrm{~F}_{4} \\
& F(2,3)-F(2,6)=\mathrm{I}(2,7)=F(2,9)-F(2,11)=\mathrm{F}(2,13)=\mathrm{F}(2,14) \\
& =F(2,15)=0 \\
& \mathrm{~F}(3,1)=0.790352 \mathrm{~F}_{1}+0.453914 \mathrm{~F}_{4} \\
& F(3,3)=K_{2}+0.686361 F_{1}+0.74091 F_{3}+0.146195 F_{4} \\
& F(3,4)=0.291711 \mathrm{~F}_{4} \\
& F(3,5)=0.807323 \mathrm{~F}_{3} \\
& F(3,9)=0.382731 \mathrm{~F}_{4} \\
& F(3,11)=0.389272 F_{I}+0.260330 \mathrm{~F}_{4} \\
& F(3,13)=0.357105 F_{3} \\
& F(3,2)-F(3,6)=F(3,7)=F(3,8)=F(3,10)=F(3,12)=F(3,14) \\
& =F(3,15)=0
\end{aligned}
$$

$$
\begin{aligned}
& F(4,1)=0.715217 F_{2}+0.456205 F_{4} \\
& F(4,2)=0.807323 F_{3} \\
& F(4,3)=0.291711 F_{4} \\
& F(4,4)=K_{3}+0.604352 F_{2}+0.739124 F_{3}+0.123081 F_{4}+0.681341 F_{5} \\
& F(4,6)=0.652746 F_{5} \\
& F(4,9)=0.420632 F_{2}+0.261129 F_{4}
\end{aligned}
$$




$$
\begin{aligned}
& F(4,10)=0.378075 F_{3} \\
& F(4,11)=0.354512 F_{4} \\
& F(4,14)=0.475860 \mathrm{~F}_{5} \\
& F(4,5)=F(4,7)=F(4,8)=P(4,12)=F(4,13)=F(4,15)=0 \\
& F(5,1)=0.715217 \mathrm{~F}_{2}+0.456285 \mathrm{~F}_{4} \\
& F(5,2)=0.291711 \mathrm{~F}_{4} \\
& F(5,3)=0.80732 .5 F_{3} \\
& F(5,5)=K_{3}+0.604352 F_{2}+0.789124 F_{3}+0.123081 F_{4}+0.681341 F_{5} \\
& F(5,7)=0.652746 F_{5} \\
& F(5,8)=0.354512 \mathrm{~F}_{4} \\
& F(5,12)=0.420632 F_{2}+0.261129 F_{4} \\
& F(5,13)=0.378075 \mathrm{~F}_{3} \\
& F(5,15)=0.475860 \mathrm{~F}_{5} \\
& F(5,4)=F(5,6)=F(5,9)=F(5,10)=F(5,11)=F(5,14)=0 \\
& F(6,4)=0.652746 \mathrm{~F}_{5} \\
& \mathrm{~F}(6,6)=\mathrm{K}_{4} * 0.487659 \mathrm{~F}_{5} \\
& F(6,14)=0.378217 F_{5} \\
& F(6,1)=F(6,2)=F(6,3)=F(6,5)=F(6,7)=F(6,8)=F(6,9) \\
& =F(6,10)=F(6,11)-F(6,12)-F(6,13)-F(6,15)=0 \\
& F(7,5)=0.652746 F_{5} \\
& F(7,7)=K_{4}+0.487659 F_{5} \\
& F(7,15)=0.378217 F_{5} \\
& F(7,1)=F(7,2)=F(7,3)=F(7,4)=F(7,6)=F(7,8)=F(7,9) \\
& =F(7,10)=F(7,11)=F(7,12)=F(7,13)-F(7,14)=0
\end{aligned}
$$




$$
\begin{aligned}
& F(8,1)=0.450175 \mathrm{~F}_{1}+0.881856 \mathrm{~F}_{4} \\
& \mathrm{~F}(8,2)=0.389272 \mathrm{~F}_{1}+0.260330 \mathrm{~F}_{4} \\
& F(8,5)=0.354512 \mathrm{~F}_{4} \\
& \mathrm{~F}(\mathrm{8}, 8)=\mathrm{H}_{1}+0.38362 \mathrm{~F}_{1}+0.85021 \mathrm{~F}_{4} \\
& F(8,12)=0.807935 F_{4} \\
& F(8,3)=F(8,4)=F(8,6)=F(8,7)=F(8,9)=F(8,10)=F(8,11) \\
& =F(8,13)=F(8,14)=F(8,15)=0 \\
& \mathrm{~F}(9,1)=0.471212 \mathrm{~F}_{2}+0.885833 \mathrm{~F}_{4} \\
& F(9,3)=0.382731 \mathrm{k}_{4} \\
& \mathrm{~F}(9,4)=0.420652 \mathrm{~F}_{2}+0.261129 \mathrm{~F}_{4} \\
& \mathrm{~F}(9,9)=\mathrm{H}_{2}+0.438769 \mathrm{~F}_{2}+0.858308 \mathrm{~F}_{4} \\
& F(9, I I)=0.807935 \mathrm{~F}_{4} \\
& F(9,2)=F(9,5)=F(9,6)=F(9,7)=F(9,8)=F(9,10)=F(9,12) \\
& \text { - } F(9,13)-F(9,14)=F(2,15)=0 \\
& F(10,2)=0.357105 F_{5} \\
& F(10,4)=0.378075 \mathrm{~F}_{5} \\
& \mathrm{~F}(10,10)=\mathrm{H}_{3}+0.291101 \mathrm{~F}_{3} \\
& P(10,1)=F(10,3)-F(10,5)=F(10,6)=F(10,7)=F(10,8) \\
& =F(10,9)=F(10,11)=F(10,12)=F(10,13)=F(10,14) \\
& =F(10,15)=0 \\
& F(11,1)=0.450175 P_{1}+0.881856 P_{4} \\
& \mathrm{~F}(11,3)=0.389272 \mathrm{~F}_{1}+0.260330 \mathrm{~F}_{4} \\
& F(11,4)=0.354512 F_{4}
\end{aligned}
$$




$$
\begin{aligned}
& F(11,9)=0.807935 F_{4} \\
& F(11,11)=H_{1}+0.38362 F_{1}+0.860210 F_{4} \\
& F(11,2)=F(11,5)=F(11,6)=F(11,7)=F(11,8)=F(11,10) \\
& \text { - } F(11,12)=F(11,13)=F(11,14)=F(11,15)=0 \\
& F(12,1)=0.471212 F_{2}+0.885835 F_{4} \\
& F(12,2)=0.382731 \mathrm{~F}_{4} \\
& F(12,5)=0.420632 F_{2}+0.261129 F_{4} \\
& F(12,8)=0.307935 F_{4} \\
& F(12,12)=H_{2}+0.438769 \mathrm{I}_{2}+0.868303 \mathrm{~F}_{4} \\
& F(12,3)=F(12,4)=F(12,6)=F(12,7)-F(12,9)=F(12,10) \\
& =F(12,11)=F(12,13)=F(12,14)=F(12,15) \propto 0 \\
& F(13,3)=0.357105 F_{3} \\
& \mathrm{~F}(13,5)=0.378075 \mathrm{~F}_{3} \\
& F(13,13)=H_{3}+0.291101 \mathrm{~F}_{3} \\
& F(13,1)=F(13,2)=F(13,4)=F(13,6)-F(13,7)-F(13,8)=F(13,9) \\
& =F(13,10)=F(13,11)=F(13,12)-F(13,14)=F(13,15)=0 \\
& F(14,4)=0.475860 F_{5} \\
& \mathrm{~F}(14,6)=0.373217 \mathrm{~F}_{5} \\
& F(14,14)=\mathrm{H}_{4}+0.428923 \mathrm{~F}_{5} \\
& F(14,1)=F(14,2)=F(14,3)=F(14,5)=F(14,7)=F(14,8)=F(14,9) \\
& =F(14,10)=F(14,11)=F(14,12)=F(14,13)-F(14,15)=0
\end{aligned}
$$




$$
\begin{aligned}
F(15,5) & =0.475800 \mathrm{~F}_{5} \\
F(15,7) & =0.378217 \mathrm{~F}_{5} \\
F(15,15) & =H_{4}+0.428923 F_{5} \\
F(15,1) & =F(15,2)=F(15,3)=F(15,4)=F(15,6)=F(15,8) \\
& =F(15,9)=F(15,10)=F(15,11)=F(15,12)=F(15,13) \\
& =F(15,14)=0
\end{aligned}
$$

16.4 F-natrix of gascous oxalic acid (1st model) in symetry co-ordinates

$$
\begin{aligned}
& F(1,1)=F_{1}+1.61801 F_{1}+1.407947 F_{2}+1.984183 F_{4} \\
& F(1,2)=1.118434 F_{1}+0.656073 F_{4} \\
& F(1,3)=1.011470 F_{2}+0.645284 F_{4} \\
& F(1,4)=0 \\
& F(1,5)=-0.259609 F_{1}-0.272054 F_{2}-1.020576 F_{4} \\
& F(1,6)=-0.450175 F_{1}+0.471212 F_{2}+0.003977 F_{4} \\
& F(1,7)=0 \\
& F(1,8)=F(1,9)=F_{1}(1,10)=F(1,11)=F(1,12)=F(1,13)=0 \\
& F(2,1)=1.118434 F_{1}+0.656073 F_{4} \\
& F(2,2)=F_{2}+0.686361 F_{1}+0.74091 F_{3}+0.146195 F_{5} \\
& F(2,3)=0.807523 F_{3}+0.291711 F_{4} \\
& F(2,4)=0 \\
& F(2,5)=-0.15892 F_{1}+0.291575 F_{3}-0.262529 F_{4} \\
& F(2,0)=-0.275257 F_{1}+0.08655 F_{4} \\
& F(2,7)=0 \\
& F(2,8)=F(2,9)=F(2,10)=F(2,11)=F(2,12)=F(2,13)=0
\end{aligned}
$$




$$
\begin{aligned}
& F(3,1)=1.01147 F_{2}+0.645284 F_{4} \\
& \mathrm{I}(3,2)=0.807323 \mathrm{~F}_{3}+0.291711 \mathrm{~F}_{4} \\
& F(3,3)=x_{3}+0.604352 F_{2}+0.789124 F_{3}+0.123081 F_{4}+0.681341 F_{5} \\
& R(3,4)=0.652746 \mathrm{~F}_{5} \\
& F(3,5)=-0.171722 F_{2}+0.308697 F_{3}-0.251334 F_{4} \\
& F(3,0)=0.297432 F_{2}-0.066032 F_{4} \\
& F(3,7)=0.47586 F_{5} \\
& F(3,8)=F(3,9)=\nabla(3,10)=F(3,11)=F(3,12)-F(3,13)=0 \\
& F(4,1)=0 \\
& F(4,2)=0 \\
& F(4,3)=0.652746 P_{5} \\
& r(4,4)=K_{4}+0.487659 \mathrm{r}_{5} \\
& F(4,5)=0 \\
& F(4,0)=0 \\
& F(4,7)=0.376217 F_{5} \\
& F(4,8)=F(4,9)=F(4,10)=F(4,11)=F(4,12)=F(4,13)=0 \\
& F(5,1)=-0.259009 F_{1}-0.272054 F_{2}-1.020576 F_{4} \\
& F(5,2)=-0.15892 F_{1}+0.291575 F_{3}-0.262529 F_{4} \\
& F(5,3)=-0.171722 F_{2}+0.303697 F_{3}-0.251334 F_{4} \\
& F(5,4)=0 \\
& F(5,5)=\left(H_{1}\right)_{/ 6}+\left(H_{2}\right)_{/ 6}+\frac{2}{3} H_{3}+0.063937 F_{1}+0.073128 F_{2}+0.194057 F_{3} \\
& +0.557395 \mathrm{~F}_{4} \\
& F(5,0)=\frac{H_{1}}{273}-\frac{H_{2}}{275}+0.110742 F_{1}-0.126662 F_{2}-0.002338 F_{4} \\
& F(5,7)=0 \\
& F(5,8) \quad \approx F(5,9)=F(5,10)-F(5,11)=F(5,12)=F(5,13)=0
\end{aligned}
$$




$$
\begin{aligned}
& F(6,1)=-0.450175 \mathrm{~F}_{1}+0.471212 \mathrm{~F}_{2}+0.003977 \mathrm{~F}_{4} \\
& F(0,2)=-0.275257 F_{1}+0.08655 F_{4} \\
& F(6,3)=0.297632 F_{2}-0.056032 F_{4} \\
& F(6,4)=0 \\
& F(6,5)=\frac{H_{1}}{273}-\frac{H_{2}}{273}+0.110742 F_{1}-0.126662 F_{2}-0.002338 F_{4} \\
& F(0,0)=\frac{H_{1}}{2}+\frac{l_{2}}{2}+0.19181 \mathrm{~F}_{1}+0.219385 \mathrm{~F}_{2}+0.056324 \mathrm{~F}_{4} \\
& F(6,7)=0 \\
& F(6,8)=F(6,9)=F(6,10)=F(6,11)=F(6,12)=F(6,13)=0 \\
& P(7,1)=0 \\
& F(7,2)=0 \\
& \mathrm{~F}(7,3)=0.47586 \mathrm{~F}_{5} \\
& F(7,4) \quad 0.378217 \mathrm{~F}_{5} \\
& F(7,5)=0 \\
& F(7,6)=0 \\
& \mathrm{~F}(7,7) \quad-\mathrm{H}_{4}+0.428923 \mathrm{~F}_{5} \\
& F(7,8)=F(7,9)=F(7,10)=F(7,11)=F(7,12)=F(7,13)=0 \\
& F(8,1)=F(8,2)=F(8,3)=F(8,4)=F(8,5)=F(8,6)=F(8,7)=0 \\
& F(8,8)=F_{2}+0.686361 F_{1}+0.74091 F_{3}+0.146195 F_{4} \\
& F(8,9)=0.807323 \mathrm{~g}_{3}-0.291711 \mathrm{P}_{4} \\
& F(8,10) \quad 0 \\
& F(8,11)=-0.15892 \mathrm{~F}_{1}+0.291575 \mathrm{~F}_{3}+0.04997 \mathrm{~F}_{4} \\
& F(8,12)=-0.275257 F_{1}-0.454713 F_{4} \\
& \mathrm{~F}(8,1 ;)=0
\end{aligned}
$$




$$
\begin{aligned}
& F(9,1)=F(9,2)=F(9,3)=F(0,4)=F(0,5)=F(9,6)=F(9,7)=0 \\
& \mathrm{~F}(9,0)=0.807323 \mathrm{~F}_{3}-0.291711 \mathrm{~F}_{4} \\
& F(9,9)=K_{3}+0.604352 F_{2}+0.789124 F_{3}+0.12381 F_{4}+0.681341 F_{5} \\
& F(9,10)=0.652746 \Gamma_{5} \\
& F(9,11)=-0.171722 E_{2}+0.303697 F_{3}+0.033123 F_{4} \\
& F(9,12)=0.297432 F_{2}+0.435324 F_{4} \\
& F(9,13)=0.475860 \mathrm{p}_{5} \\
& F(10,1)=F(10,2)=F(10,3)=F(10,4)=F(10,5)=F(10,6)=F(10,7) \\
& -0 \\
& F(10,81=0 \\
& F(10,9)=0.652746 F_{5} \\
& \mathrm{~F}(10,10)=\mathrm{K}_{4}+0.487659 \mathrm{~F}_{5} \\
& F(10,11)=0 \\
& F(10,12)=0 \\
& F(10,13)=0.378217 F_{5} \\
& F(11,1)=F(11,2)=F(11,5)=F(11,4)=F(11,5)=F(11,6)=F(11,7) \\
& \text { * } 0 \\
& \mathrm{~F}(11,8)=-0.15892 \mathrm{~F}_{1}+0.291575 \mathrm{~F}_{3}+0.04997 \mathrm{~F}_{4} \\
& \mathrm{~F}(11,9)=-0.171722 \mathrm{~F}_{2} * 0.308697 \mathrm{~F}_{3}+0.058123 \mathrm{~F}_{4} \\
& F(11,10)=0 \\
& \mathrm{~F}(11,11)=\frac{\mathrm{H}_{1}}{6}+\frac{\mathrm{H}_{2}}{6}+\frac{2}{3} \mathrm{H}_{3}+0.053937 \mathrm{~F}_{1}+0.073128 \mathrm{~F}_{2}+0.194057 \mathrm{~F}_{3} \\
& \begin{aligned}
& +0.018775 F_{4} \\
F(17,12)= & \frac{i_{1}}{273}-\frac{H_{1}}{273}+0.110742 F_{1}-0.126662 F_{2}-0.002338 F_{4}
\end{aligned} \\
& F(11,15)=0
\end{aligned}
$$




$$
\begin{aligned}
& F(12,1)=F(12,2)=F(12,3)=F(12,4)=F(12,5)=F(12,6) \\
& =F(12,7)=0 \\
& F(12,8)=-0.275257 \mathrm{~F}_{1}-0.454713 \mathrm{~F}_{4} \\
& F(12,9)=0.297432 F_{2}+0.435324 F_{4} \\
& \mathrm{~F}(12,10)=0 \\
& F(12,11)=\frac{H_{1}}{2 \sqrt{3}}-\frac{H_{2}}{2 \sqrt{3}}+0.110742 F_{1}-0.126662 F_{2}-0.002338 F_{4} \\
& F(12,12)=\frac{H_{1}}{2}=\frac{H_{2}}{2}+0.19181 \mathrm{~F}_{1}+0.219385 \mathrm{~F}_{2}+1.672194 \mathrm{~F}_{4} \\
& F(12,13)=0 \\
& F(13,1)=F(13,2)=F(13,3)=F(13,4)=F(13,5)=F(13,6)=F(13,7) \\
& =0 \\
& F(13,8)=0 \\
& F(13,9)=0.475860 F_{5} \\
& F(13,10)=0.378217 F_{5} \\
& F(13,11)=0 \\
& F(13,12)=0 \\
& F(13,13)=H_{4}+0.428923 F_{5}
\end{aligned}
$$




\section{CAAPTER 17}

\section{G-MATRIX OF TIE INPLANE VBRATIONS}

OF GASEOUS OXUIC ACTD $2 \mathrm{ND}$ IODEL

\section{I Evaluation of the B-matrix of the laplane vibration of gaseous}

oxalic acid 2nid model

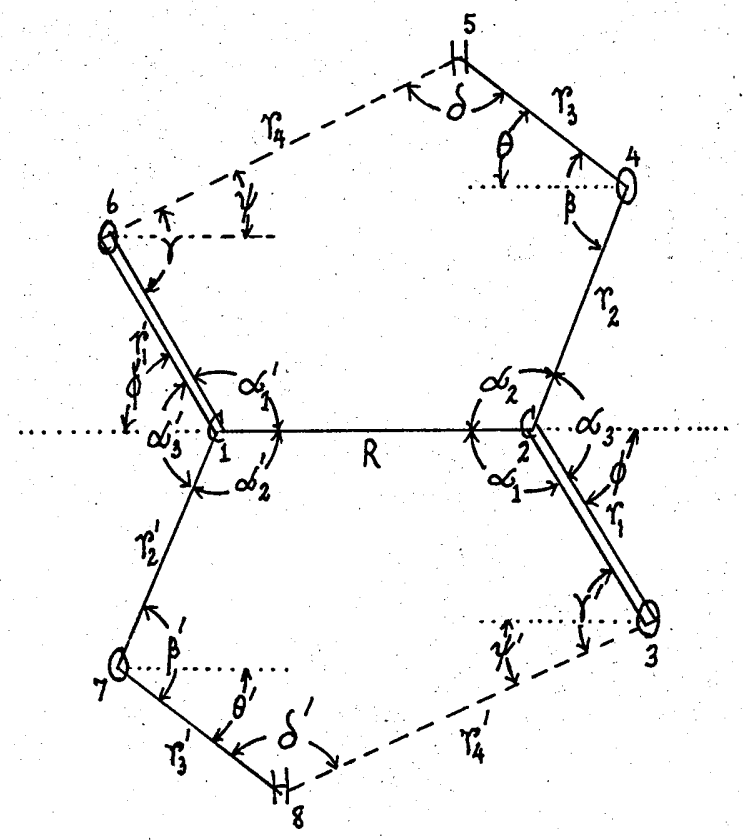

\section{Fig. 21}

In order to simplify tho problen eaci aton of gaseous oxalic acid is labelled by a number as in Fig. 21. For gaseous oxalic acid 2nd model there are altogether 21. intemal co-ordinates, 9 are bond stretching co-ordinates and 12 are angle bending co-orüinates, as follows:- 
Bond stretching co-ordinates

$\Delta R=\quad$ change in $C-C$ distance

$\Delta \mathrm{r}_{1}=\Delta \mathrm{r}_{1}=$ change in $\mathrm{C}=0$ distance

$\Delta \mathrm{r}_{2}=\Delta \mathrm{r}_{2}{ }_{2}=$ chenge in $\mathrm{C}-0$ distanco

$\Delta r_{3}=\Delta r_{3}^{\prime}=$ change in $0-\mathrm{H}$ distanco

$\Delta r_{4}=\Delta r_{4}^{\prime}=$ change in $0 \ldots H$ distance

Angle bending comorainetes

$\Delta \alpha_{1}=\Delta \alpha^{\prime}{ }_{1}$ change in $C-C=0$ angle

$\Delta \alpha_{2}=\Delta a_{2}^{\prime}$ - change in $C-C-0$ angle

$\Delta a_{3}=\Delta e^{\prime}{ }_{3}$ change in $0-C-O$ angle

$\Delta B^{\prime}=\Delta B^{\prime}=$ chango in $C-O-H$ anglo

$\Delta \delta=\Delta \delta^{\prime}=$ change in $0-H \ldots 0$ angle

$\Delta \gamma=\Delta \gamma$ : change in $C=0 \ldots H$ angle

The B-matrix elements are evaluated using Wilson's vector method (see section 5.2) and the results are

B-matrix of tine inplane vibration of gasoous oxalic acid 2nd model

$$
\begin{aligned}
& \left(\Delta R, \Delta x_{1}\right)=-1 \\
& \left(\Delta R, \Delta x_{2}\right)=1 \\
& \left(\Delta r_{1}, \Delta x_{2}\right)=-\cos \phi \quad\left(\Delta x_{2}, \Delta x_{2}\right)=-\cos \left(\alpha_{3}-\phi\right) \\
& \left(", \Delta y_{2}\right)=\sin \phi \quad\left(", \Delta y_{2}\right)=-\sin \left(\alpha_{3}-\phi\right) \\
& \left(", \Delta x_{3}\right)=\cos \phi \quad\left(", \Delta x_{4}\right)=\cos \left(\alpha_{3}-\phi\right) \\
& \left(", \Delta y_{3}\right)=-\sin \phi \quad\left(", \Delta y_{4}\right)=\sin \left(\alpha_{3}-\phi\right)
\end{aligned}
$$




$$
\begin{aligned}
& \left(\Delta r_{3}, \Delta x_{4}\right)=\cos \theta \quad\left(\Delta r_{4}, \Delta x_{5}\right)=\cos 4 \\
& \left(" \Delta y_{4}\right)=-\sin \theta \quad\left(", \Delta y_{5}\right)=\sin \psi \\
& \left(", \Delta x_{5}\right)=-\cos \theta \quad\left(", \Delta x_{6}\right)=-\cos \psi \\
& \left(", \Delta y_{5}\right)=\sin \theta \quad\left(", \Delta y_{6}\right)=-\sin \psi \\
& \left(\Delta \mathrm{r}_{1}^{\prime}, \Delta \mathrm{x}_{1}\right)=\cos \quad\left(\Delta \mathrm{r}_{2}^{\prime}, \Delta \mathrm{x}_{1}\right)=\cos \left(\alpha_{3}-\phi\right) \\
& \left(", \Delta y_{1}\right)=-\sin \phi \quad\left(", \Delta y_{1}\right)=\sin \left(a_{3}-\phi\right) \\
& \text { ( " } \left., \Delta x_{6}\right)=-\cos \phi \quad\left(", \Delta x_{7}\right)=-\cos \left(\alpha_{3}-\phi\right) \\
& \text { ( " } \left.{ }^{\prime} \Delta y_{6}\right)=\sin \quad\left(", \Delta y_{7}\right)=-\sin ^{\prime}\left(a_{5}-\phi\right) \\
& \left(\Delta \mathrm{r}_{3}^{\prime}, \Delta \mathrm{x}_{7}\right)=-\cos \theta^{\prime} \quad\left(\Delta \mathrm{r}_{4}^{\prime}, \Delta \mathrm{x}_{3}\right)=\cos \psi \\
& \left(", \Delta y_{7}\right)=\sin \theta^{\prime} \quad\left(", \Delta y_{3}\right)=\sin \psi \\
& \left(", \Delta x_{8}\right)=\cos \theta^{\prime} \quad\left(", \Delta x_{8}\right)=-\cos \psi \\
& \left(", \Delta y_{8}\right)=-\sin \theta^{\prime} \quad\left(", \Delta y_{8}\right)=-\sin \psi \\
& r_{1}\left(\Delta a_{1}, \Delta x_{1}\right)=0 \\
& r_{2}\left(\Delta a_{2}, \Delta x_{1}\right)=0 \\
& r_{1}\left(", \Delta y_{1}\right)=r_{1} / R \\
& r_{2}\left(", \Delta y_{1}\right)=-r_{2} / R \\
& r_{1}\left(" \Delta x_{2}\right)=-\sin \phi \\
& r_{2}\left(", \Delta x_{2}\right)=-\sin \left(a_{3}-\phi\right) \\
& r_{1}\left(", \Delta y_{2}\right)=-\cos \phi-\left(r_{1} / R\right) \quad r_{2}\left(", \Delta y_{2}\right)=\left(r_{2} / R\right)+\cos \left(a_{3}-\phi\right) \\
& r_{1}\left(" \Delta x_{3}\right)=\sin \quad \quad r_{2}\left(", \Delta x_{4}\right)=\sin \left(a_{3}-\phi\right) \\
& r_{1}\left(", \Delta y_{3}\right)=\cos \phi \quad r_{2}\left(", \Delta y_{4}\right)=-\cos \left(\alpha_{3}-\phi\right) \\
& \sqrt{r_{1} r_{2}}\left(\Delta \alpha_{3}, \Delta x_{2}\right)=\left\{r_{2} \sin \phi+r_{1} \sin \left(a_{3}-\phi\right)\right\} / \sqrt{r_{1} r_{2}} \\
& \sqrt{r_{1} r_{2}}\left(", \Delta y_{2}\right)=\left\{r_{2} \cos \phi-r_{1} \cos \left(a_{3}-\phi\right)\right\} / \sqrt{r_{1} r_{2}} \\
& \sqrt{r_{1} r_{2}}\left(", \Delta x_{3}\right)=-r_{2} \sin \phi / \sqrt{r_{1} r_{2}} \\
& \sqrt{r_{1} r_{2}}\left(", \Delta y_{3}\right)=-r_{2} \cos \phi / \sqrt{r_{1} r_{2}} \\
& \sqrt{x_{1} x_{2}}\left(" \Delta \Delta x_{4}\right)=-x_{1} \sin \left(a_{3}-\phi\right) / \sqrt{x_{1} r_{2}} \\
& \sqrt{r_{1} r_{2}}\left(", \Delta y_{4}\right)=r_{1} \cos \left(\alpha_{3}-\phi\right) / \sqrt{r_{1} r_{2}}
\end{aligned}
$$




$$
\begin{aligned}
& x_{1}^{\prime}\left(\Delta \alpha^{\prime}{ }_{1}, \Delta x_{1}\right)=\sin \phi^{\prime} \\
& r^{\prime}{ }_{1}\left(", \Delta y_{1}\right)=\left(r^{\prime} / R\right)+\cos \phi^{\prime} \\
& r_{1}\left(n, \Delta x_{2}\right)=0 \\
& r_{1}^{\prime}\left(" \Delta y_{2}\right)=-r_{1}^{\prime} / R \\
& r_{1}^{\prime}\left(", \Delta x_{6}\right)=-\sin \phi^{\prime} \\
& r_{1}\left(", \Delta y_{6}\right)=-\cos \phi^{\prime} \\
& r_{2}^{\prime}\left(\Delta \alpha_{2}^{\prime}, \Delta x_{1}\right)=\sin \left(\alpha^{\prime}{ }_{3}-\phi\right) \\
& r_{2}^{\prime}\left(", \Delta y_{1}\right)=-\cos \left(\alpha^{\prime}{ }_{3}-\phi^{\prime}\right)-\left(r_{2}^{\prime} / R\right) \\
& r_{2}{ }^{\prime}\left(", \Delta x_{2}\right)=0 \\
& r_{2}^{\prime}\left(*, \Delta y_{2}\right)=r_{2}{ }_{2} / R \\
& r_{2}^{\prime}\left(" \Delta x_{7}\right)--\sin \left(a^{\prime}-\phi^{\prime}\right) \\
& r_{2}^{\prime}\left(" \Delta y_{7}\right)=\cos \left(\alpha^{\prime}{ }_{3}-\phi^{\prime}\right) \\
& \sqrt{r^{\prime}{ }_{1} r^{\prime}}{ }_{2}\left(\Delta \alpha^{\prime}{ }_{3}, \Delta x_{1}\right)=-\left\{r^{\prime}{ }_{2} \sin \phi^{\prime}+r^{\prime}{ }_{1} \sin \left(\alpha^{\prime}{ }_{3}-\phi^{\prime}\right)\right\} / \sqrt{r^{\prime}{ }_{1} r^{\prime}{ }_{2}} \\
& \sqrt{r^{\prime}{ }_{1} r_{2}^{\prime}}\left(" \Delta y_{1}\right)=\left(r^{\prime}{ }_{1} \cos \left(\alpha^{\prime}{ }_{3}-\phi^{\prime}\right)-r^{\prime}{ }_{2} \cos \phi^{\prime}\right) / \sqrt{r^{\prime}{ }_{1} r^{\prime \prime}} \\
& \sqrt{r^{\prime}{ }_{1} r_{2}^{\prime}}\left(", \Delta x_{6}\right)=r^{\prime}{ }_{2} \sin \phi^{\prime} / \sqrt{r^{\prime}{ }_{2} r_{2}^{\prime}} \\
& \sqrt{r^{\prime}{ }_{1} r^{\prime}}{ }_{2}\left(", \Delta y_{6}\right)=r^{\prime}{ }_{2} \cos \phi^{\prime} / \sqrt{r^{\prime}{ }_{1} r^{\prime}} \\
& \sqrt{r^{\prime}{ }_{1} r_{2}^{\prime}}\left(" ; \Delta x_{7}\right)=r^{\prime}{ }_{1} \sin \left(\alpha^{\prime}{ }_{3}-\phi^{\prime}\right) / \sqrt{r^{\prime}{ }_{1} r^{\prime}} \\
& \sqrt{r^{\prime}{ }_{1} r_{2}^{\prime}}\left(n, \Delta y_{7}\right)=-r^{\prime}{ }_{1} \cos \left(a^{\prime}{ }_{3}-\phi^{\prime}\right) / \sqrt{x^{\prime}{ }_{1} r^{\prime}} \\
& \sqrt{r_{2}} r_{3}\left(\Delta \beta, \Delta x_{2}\right)=r_{3} \sin (\beta-\theta) / \sqrt{r_{2} r_{3}} \\
& "\left(", \Delta y_{2}\right)=-r_{3} \cos (\beta-\theta) / \sqrt{r_{2} r_{3}} \\
& \text { " (". } \left.\Delta x_{4}\right)--\left\{r_{2} \sin \theta+r_{3} \sin (\theta-\theta)\right\} / \sqrt{r_{2} r_{3}} \\
& \text { " }\left(", \Delta y_{4}\right)=\left\{r_{3} \cos (\beta-\theta)-r_{2} \cos \theta\right\} / \sqrt{r_{2} r_{3}} \\
& \text { " }\left(" \Delta x_{5}\right)=r_{2} \sin \theta / \sqrt{r_{2} r_{3}} \\
& \text { * (",Ay })=r_{2} \cos \theta / \sqrt{r_{2} r_{3}}
\end{aligned}
$$




$$
\begin{aligned}
& \sqrt{r_{3} r_{4}}\left(\Delta \delta, \Delta x_{4}\right)=r_{4} \sin \theta / \sqrt{r_{3} r_{4}} \\
& \text { " }\left(" \Delta y_{4}\right)=r_{4} \cos \theta / \sqrt{r_{3} r_{4}} \\
& "\left(", \Delta x_{5}\right)=\left\{r_{3} \sin \psi-r_{4} \sin \theta\right\} / \sqrt{r_{3} r_{4}} \\
& \text { " }\left(", \Delta y_{5}\right)=-\left(r_{3} \cos \theta+r_{4} \cos \theta\right\} / \sqrt{r_{3} r_{4}} \\
& \text { " (", } \left.\Delta x_{6}\right)=-r_{3} \sin \psi / \sqrt{r_{3} x_{4}} \\
& \text { " }\left(" \Delta y_{6}\right)=r_{3} \cos \psi / \sqrt{r_{3} r_{4}} \\
& \begin{aligned}
\sqrt{r^{\prime}{ }_{1} r_{4}}\left(\Delta \gamma, \Delta x_{1}\right) & =-r_{4} \sin (\gamma-\psi) / \sqrt{r^{\prime}{ }_{1} r_{4}} \\
"\left(", \Delta y_{1}\right) & =-r_{4} \cos (\gamma-\psi) / \sqrt{r^{\prime}{ }_{1} r_{4}} \\
"\left(", \Delta x_{5}\right) & =-r^{\prime}{ }_{1} \sin \psi / \sqrt{r^{\prime}{ }_{1} r_{4}} \\
"\left(", \Delta y_{5}\right) & =r^{\prime}{ }_{1} \cos \psi / \sqrt{r^{\prime}{ }_{1} r_{4}} \\
"\left(", \Delta x_{6}\right) & =\left\{r_{4} \sin (\gamma-\psi)+r^{\prime}{ }_{1} \sin \psi\right\} / \sqrt{r^{\prime}{ }_{1} r_{4}} \\
"\left(", \Delta y_{6}\right) & =\left\{r_{4} \cos (\gamma-\psi)-r_{1}^{\prime} \cos \psi\right\} / \sqrt{r^{\prime}{ }_{1} r_{4}}
\end{aligned}
\end{aligned}
$$

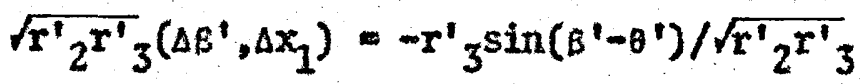

$$
\begin{aligned}
& \text { " }\left(", \Delta y_{1}\right)=r_{3}^{\prime} \cos \left(\beta^{*}-0 \cdot\right) / \sqrt{r^{\prime}{ }_{2} r_{3}^{\prime}}
\end{aligned}
$$

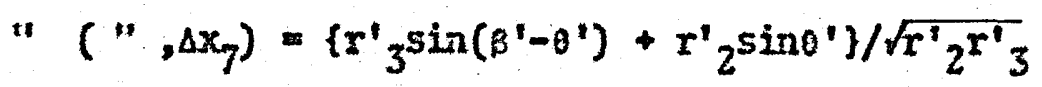

$$
\begin{aligned}
& \text { " (", } \left.\Delta y_{7}\right)=\left\{r_{2}^{\prime} \cos \theta^{\prime}-r^{\prime}{ }_{3} \cos \left(\theta^{\prime}-\theta^{\prime}\right)\right\} / \sqrt{r^{\prime}{ }_{2} r^{\prime}} \\
& \text { " (" } \left., \Delta x_{8}\right)=-r_{2}^{\prime} \sin \theta / \sqrt{r^{\prime}{ }_{2} r^{\prime}} \\
& \text { " (" } \left., \Delta y_{8}\right)=-r_{2}^{\prime} \cos \theta^{\prime} / \sqrt{r^{\prime}{ }_{2} r^{\prime}}
\end{aligned}
$$

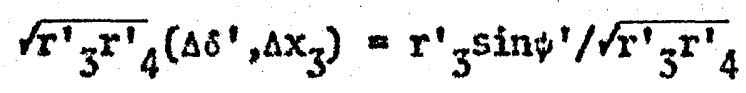

$$
\begin{aligned}
& "\left(", \Delta y_{3}\right)=-x^{\prime}{ }_{3} \cos \psi 1 / \sqrt{r^{\prime}{ }_{3}^{r^{\prime}}} \\
& \text { "(", } \left., \Delta x_{7}\right)=-x^{\prime}{ }_{4}^{\sin \theta^{1} / \sqrt{r^{\prime}} 3^{r^{\prime}} 4} \\
& \text { " (", } \left.\Delta y_{7}\right)=-r^{\prime}{ }_{4} \cos \theta 1 / \sqrt{r^{\prime}{ }^{r^{\prime}}} \\
& \text { " ( " } \left., \Delta x_{8}\right)=\left\{r^{\prime}{ }_{4} \sin \theta^{\prime}-r^{\prime}{ }_{3} \sin \phi^{\prime}\right\} / \sqrt{r^{\prime}{ }_{3}{ }^{\prime}{ }_{4}} \\
& \text { " ( " } \left., \Delta y_{8}\right)=\left\{r^{\prime}{ }_{4} \cos \theta^{\prime}+r^{\prime}{ }_{3} \cos \psi^{\prime}\right\} / \sqrt{r^{\prime}{ }_{3} r^{\prime}}
\end{aligned}
$$




$$
\begin{aligned}
\sqrt{r_{1} r^{\prime}}\left(\Delta r^{\prime}, \Delta x_{2}\right) & =r_{4}^{\prime} \sin \left(\gamma^{\prime}-\phi^{\prime}\right) / \sqrt{r_{1} r_{4}^{\prime}} \\
"\left(", \Delta y_{2}\right) & =r^{\prime}{ }_{4} \cos \left(\gamma^{\prime}-\psi^{\prime}\right) / \sqrt{r_{1} r^{\prime}} \\
"\left(", \Delta x_{3}\right) & =-\left\{r_{1} \sin \psi^{\prime}+r^{\prime}{ }_{4} \sin \left(r^{\prime}-\phi^{\prime}\right)\right\} / \sqrt{r_{1} r^{\prime}} \\
"\left(", \Delta y_{3}\right) & =\left\{r_{1} \cos \psi^{\prime}-r^{\prime}{ }_{4} \cos \left(\gamma^{\prime}-\psi^{\prime}\right)\right\} / \sqrt{r_{1} r^{\prime}} \\
"\left(", \Delta x_{8}\right) & =+r_{1} \sin \psi^{\prime} / \sqrt{r_{1} r^{\prime}} \\
"\left(", \Delta y_{8}\right) & =-r_{1} \cos \psi^{\prime} / \sqrt{r_{1} r_{4}^{\prime}}
\end{aligned}
$$

All other elements are zero.

17.2 D-matrix of the inplane vibration of gaseous oxalic acid 2 nd model

$$
\begin{array}{ll}
\left(\Delta R, \Delta x_{1}\right)=-\sqrt{\mu_{c}} & \\
\left(\Delta R, \Delta x_{2}\right)=\sqrt{\mu_{c}} & \left(\Delta x_{2}, \Delta x_{2}\right)=-\sqrt{\mu_{c}} \cos \left(a_{3}-\phi\right) \\
\left(\Delta r_{1}, \Delta x_{2}\right)=-\sqrt{\mu_{c}} \cos \phi & \left(", \Delta y_{2}\right)=-\sqrt{\mu_{c}} \sin \left(\alpha_{3}-\phi\right) \\
\left(", \Delta y_{2}\right)=\sqrt{\mu_{c}} \sin \phi & \left(", \Delta x_{4}\right)=\sqrt{\mu_{0}} \cos \left(\alpha_{3}-\phi\right) \\
\left(", \Delta x_{3}\right)=\sqrt{\mu_{0}} \cos \phi & \left(", \Delta y_{4}\right)=\sqrt{\mu_{0}} \sin \left(\alpha_{3}-\phi\right) \\
\left(", \Delta y_{3}\right)=-\sqrt{\mu_{0}} \sin \phi & \left(\Delta x_{4}, \Delta x_{5}\right)=\sqrt{\mu_{11}} \cos \psi \\
\left(\Delta r_{3}, \Delta x_{4}\right)=\sqrt{\mu_{0}} \cos \theta & \left(", \Delta y_{5}\right)=\sqrt{\mu_{1}} \sin \psi \\
\left(", \Delta y_{4}\right)=-\sqrt{\mu_{0}} \sin \theta & \left(", \Delta x_{6}\right)=-\sqrt{\mu_{0}} \cos \psi \\
\left(", \Delta x_{5}\right)=-\sqrt{\mu_{11}} \cos \theta & \left(", \Delta y_{6}\right)=-\sqrt{\mu_{0}} \sin \psi \\
\left(", \Delta y_{5}\right)=\sqrt{\mu_{11}} \sin \theta & \left(\Delta r^{\prime}, \Delta x_{1}\right)=\sqrt{\mu_{c}} \cos \left(\alpha_{3}-\phi\right) \\
\left(\Delta r^{\prime}, \Delta x_{1}\right)=\sqrt{\mu_{c}} \cos \phi & \left(" \Delta y_{1}\right)=\sqrt{\mu_{c}} \sin \left(\alpha_{3}-\phi\right) \\
\left(", \Delta y_{1}\right)=-\sqrt{\mu_{c}} \sin \phi & \left(" \Delta x_{7}\right)=-\sqrt{\mu_{0}} \cos \left(\alpha_{3}-\phi\right) \\
\left(", \Delta x_{6}\right)=-\sqrt{\mu_{0}} \cos \phi & \left(" \Delta y_{7}\right)=-\sqrt{\mu_{0}} \sin \left(\alpha_{3}-\phi\right) \\
\left(", \Delta y_{6}\right)=\sqrt{\mu_{0}} \sin \phi & \left(",{ }^{\prime \prime}\right.
\end{array}
$$




$$
\begin{array}{ll}
\left(\Delta r^{\prime}, \Delta x_{7}\right)=-\sqrt{\mu_{0}} \cos \theta^{\prime} & \left(\Delta r^{\prime}{ }_{4}, \Delta x_{3}\right)=\sqrt{\mu_{0}} \cos \psi \\
\left(", \Delta y_{7}\right)=\sqrt{\mu_{0}} \sin \theta^{\prime} & \left(" \Delta y_{3}\right)=\sqrt{\mu_{0}} \sin \psi \\
\left(", \Delta x_{8}\right)=\sqrt{\mu_{H}} \cos \theta^{\prime} & \left(", \Delta x_{8}\right)=-\sqrt{\mu_{H}} \cos \psi \\
\left(", \Delta y_{8}\right)=-\sqrt{\mu_{H}} \sin \theta^{\prime} & \left(" \Delta y_{8}\right)=-\sqrt{\mu_{H}} \sin \psi
\end{array}
$$

$$
\begin{aligned}
& r_{1}\left(\Delta \alpha_{1} \Delta x_{1}\right)=0 \quad r_{2}\left(\Delta \alpha_{2}, \Delta x_{1}\right)=0 \\
& "\left(" \Delta y_{1}\right)=\sqrt{\mu}_{c}\left(r_{1} / R\right) \quad "\left(", \Delta y_{1}\right)=-{\sqrt{\mu_{c}}}_{c}\left(r_{2} / R\right) \\
& \text { " (" } \left.\Delta x_{2}\right)=-\sqrt{\mu_{c}} \sin \phi \quad "\left(" \Delta x_{2}\right)=-\sqrt{\mu_{c}} \sin \left(\alpha_{3}-\phi\right) \\
& \left."\left(" \Delta y_{2}\right)=-\sqrt{\mu_{c}}\left(\cos \phi+r_{1} / R\right) "\left(", \Delta y_{2}\right)=\sqrt{u_{c}}\left(r_{2} / R\right)+\cos \left(\alpha_{3}-\phi\right)\right\} \\
& \text { "(" } \left." \Delta x_{3}\right)=\sqrt{\mu_{0}} \sin \phi \quad "\left(" \Delta x_{4}\right)=\sqrt{\mu_{0}} \sin \left(a_{3}-\phi\right) \\
& "\left(" \Delta y_{3}\right)=\sqrt{\mu_{0}} \cos \phi \quad "\left(" \Delta y_{4}\right)=-\sqrt{\mu_{0}} \cos \left(a_{3}-\phi\right)
\end{aligned}
$$

$$
\begin{aligned}
& \sqrt{r_{1} x_{2}}\left(\Delta a_{3}, \Delta x_{2}\right)=\sqrt{\omega_{c}}\left\{r_{2} \sin \phi+r_{1} \sin \left(a_{3}-\phi\right)\right\} / \sqrt{r_{1} r_{2}} \\
& "\left(", \Delta y_{2}\right)=\sqrt{u_{c}}\left(r_{2} \cos \phi-r_{1} \cos \left(\alpha_{3}-\phi\right)\right\} / \sqrt{r_{1} r_{2}} \\
& \text { " (", } \left.\Delta x_{3}\right)=-\sqrt{\mu_{0}} r_{2} \sin \notin / \sqrt{r_{1} r_{2}} \\
& \text { " (" } \left.. \Delta y_{3}\right)=-\sqrt{\mu_{0}} r_{2} \cos \phi / \sqrt{r_{1} r_{2}} \\
& "\left(", \Delta x_{4}\right)=-\sqrt{\mu_{0}} x_{1} \sin \left(\alpha_{3}-\phi\right) / \sqrt{x_{1} x_{2}} \\
& "\left(", \Delta y_{4}\right)=\sqrt{u_{0}} r_{1} \cos \left(\alpha_{3}-\phi\right) / \sqrt{r_{1} r_{2}} \\
& r_{1}^{\prime}\left(\Delta \alpha^{\prime}{ }_{1}, \Delta x_{1}\right)=\sqrt{\mu}_{c} \sin \phi^{\prime} \\
& r_{1}^{\prime}\left(", \Delta y_{1}\right)=\sqrt{\mu_{c}}\left\{\left(r^{\prime} / R\right)+\cos \phi^{\prime}\right\} \\
& I_{1}^{\prime}\left(", \Delta x_{2}\right)=0 \\
& r_{1}^{\prime}\left(", \Delta y_{2}\right)=-\sqrt{u}_{c}\left(r^{\prime}{ }_{1} / R\right) \\
& r_{1}^{\prime}\left(", \Delta x_{6}\right)=-\sqrt{\omega_{0}} \sin \phi^{\prime} \\
& x_{1}^{\prime}\left(", \Delta y_{6}\right)=-\sqrt{\mu_{0}} \cos \phi^{\prime}
\end{aligned}
$$




$$
\begin{aligned}
& r_{2}^{\prime}\left(\Delta \alpha_{2}^{\prime}, \Delta x_{1}\right)=\sqrt{\mu_{c}} \sin \left(\alpha^{\prime}{ }_{3}^{-\phi^{\prime}}\right) \\
& "\left(", \Delta y_{1}\right)=-\sqrt{\mu_{c}}\left\{\left(r_{2}^{\prime} / R\right)+\cos \left(a^{\prime}{ }_{3}-\phi^{\prime}\right)\right\} \\
& \text { "( }\left(" \Delta x_{2}\right)=0 \\
& \text { " }\left(", \Delta y_{2}\right)={\sqrt{\mu_{c}}}_{c}\left(r^{\prime}{ }_{2} / R\right) \\
& "\left(" \Delta, \Delta x_{7}\right)=-\sqrt{\mu_{0}} \sin \left(\alpha^{\prime}-\phi^{\prime}\right) \\
& "\left(" \Delta y_{7}\right)=\sqrt{\mu_{0}} \cos \left(a^{\prime}{ }_{3}-\phi^{\prime}\right) \\
& \sqrt{r^{\prime}{ }_{1} r_{2}^{\prime}}{ }_{2}\left(\Delta \alpha^{\prime}{ }_{3}, \Delta x_{1}\right)=-\sqrt{11} c\left(r^{\prime}{ }_{2} \sin \phi^{\prime}+r^{\prime}{ }_{1} \sin \left(\alpha^{\prime}{ }_{3}-\phi^{\prime}\right)\right\} / \sqrt{r^{\prime}{ }_{1} r^{\prime}} \\
& "\left(", \Delta y_{1}\right)=\sqrt{\mu_{c}}\left(x^{\prime}{ }_{1} \cos \left(\alpha^{\prime}{ }_{3}-\phi^{\prime}\right)-r^{\prime}{ }_{2} \cos \phi^{\prime}\right\} / \sqrt{r^{\prime}{ }_{1} x^{\prime}} \\
& \Rightarrow \quad\left(" \Delta x_{6}\right)=\sqrt{\mu_{0}} r_{2}{ }_{2} \sin \phi / \sqrt{r^{\prime}{ }_{1}{ }^{\prime}}{ }_{2} \\
& "\left(", \Delta y_{6}\right)=\sqrt{\mu_{0}} r_{2}^{\prime} \cos \phi^{\prime} / \sqrt{r^{\prime}{ }_{1} r_{2}} \\
& " \quad\left(", \Delta x_{7}\right)=\sqrt{\mu_{0}} r_{1}^{\prime} \sin \left(\alpha_{3}^{\prime}-\phi^{\prime}\right) / \sqrt{x^{\prime}{ }_{1} r_{2}^{\prime}} \\
& \text { " } \quad\left(" \Delta y_{7}\right)=-\sqrt{\mu_{0}} r^{\prime}{ }_{1} \cos \left(\alpha^{\prime}{ }_{3}-\phi^{\prime}\right) / \sqrt{r^{\prime}{ }_{1} r^{\prime}} \\
& \sqrt{r_{2} r_{3}}\left(\Delta A, \Delta x_{2}\right)=\sqrt{\mu_{c}} r_{3} \sin (\beta-\theta) / \sqrt{r_{2} r_{3}} \\
& \text { " (", } \left.\Delta y_{2}\right)=-\sqrt{\mu_{c}} r_{3} \cos (\beta-\theta) / \sqrt{r_{2} r_{3}} \\
& "\left(", \Delta x_{4}\right)=-\sqrt{\mu_{0}}\left(r_{2} \sin \theta+r_{3} \sin (\beta-\theta)\right\} / \sqrt{r_{2} T_{3}} \\
& "\left(", \Delta y_{4}\right)=\sqrt{b_{0}}\left(r_{3} \cos (\theta-\theta)-r_{2} \cos \theta\right) / \sqrt{r_{2} r_{3}} \\
& \text { " (", } \left.\Delta x_{5}\right)=\sqrt{\mu_{H}} r_{2} \sin \theta / \sqrt{r_{2} r_{3}} \\
& \text { "( } \left.", \Delta y_{5}\right)=\sqrt{\mu_{11}} r_{2} \cos \theta / \sqrt{r_{2} r_{3}} \\
& \sqrt{r_{3} r_{4}}\left(\Delta \delta, \Delta x_{4}\right)=\sqrt{\mu_{0}} x_{4} \sin \phi / \sqrt{r_{3} r_{4}} \\
& "\left(", \Delta y_{4}\right)=\sqrt{\mu_{0}} x_{4} \cos \theta / \sqrt{r_{3} r_{4}} \\
& "\left(", \Delta x_{5}\right)=\sqrt{\mu_{H}}\left\{r_{3} \sin \psi-r_{4} \sin \theta\right\} \sqrt{r_{3} r_{4}} \\
& "\left(", \Delta y_{5}\right)=-\sqrt{\mu_{H}}\left\{x_{3} \cos \psi+r_{4} \cos \theta\right\} / \sqrt{3_{3} r_{4}} \\
& "\left(" \Delta x_{6}\right)=-\sqrt{\mu_{0}} r_{3} \sin \omega / \sqrt{r_{3} r_{4}} \\
& "\left(" \Delta{ }^{\prime} \Delta y_{6}\right)=\sqrt{\mu u} r_{3} \cos \psi / \sqrt{r_{3} r_{4}}
\end{aligned}
$$




$$
\begin{aligned}
& \sqrt{r^{\prime}{ }_{1} r_{4}}\left(\Delta \gamma, \Delta x_{1}\right)=-\sqrt{\mu_{c}} r_{4} \sin \left(\gamma-w^{\prime}\right) / \sqrt{x^{\prime}{ }_{1} r_{4}} \\
& \text { " }\left(" \Delta y_{1}\right)=-\sqrt{\mu_{c}} x_{4} \cos (\gamma-\phi) / \sqrt{r^{\prime}{ }_{1} r_{4}} \\
& "\left(", \Delta x_{5}\right)=-\sqrt{\mu_{\mathrm{H}}} \mathrm{r}_{1}^{\prime} \sin \psi / \mathrm{rr}_{1}^{\prime}{ }_{1}{ }_{4} \\
& "\left(" \Delta y_{5}\right)=\sqrt{\mu_{1}} \mathrm{r}_{1}^{\prime} \cos \psi / \sqrt{r_{1}^{\prime}{ }_{1} r_{4}} \\
& "\left(", \Delta x_{6}\right)=\sqrt{u_{0}}\left[r_{4} \sin (\gamma-\psi)+r_{1}^{\prime} \sin \psi\right\} / \sqrt{r^{\prime}{ }_{1} r_{4}} \\
& "\left(", \Delta y_{6}\right)=\sqrt{\mu_{0}}\left\{r_{4} \cos (\gamma-\psi)-r_{1}^{\prime} \cos \phi\right\} / \sqrt{r^{\prime}{ }_{1} x_{4}} \\
& \sqrt{r^{\prime}{ }_{2}^{r^{\prime}}{ }_{3}}\left(\Delta \beta^{\prime}, \Delta x_{1}\right)=-\sqrt{\mu_{c}} \mathbf{r}_{3} \sin \left(\beta^{\prime}-\theta^{\prime}\right) / \sqrt{r^{\prime} 2^{r^{\prime}} 3} \\
& \text { " } \quad\left(" \Delta y_{1}\right)=\sqrt{u_{c}} r_{3}^{\prime} \cos \left(\beta^{\prime}-\theta^{\prime}\right) / \sqrt{r^{\prime} 2^{r^{\prime}} 3} \\
& \text { " }\left(", \Delta x_{7}\right)=\sqrt{\mu_{0}}\left\{r_{3}^{\prime} \sin \left(\beta^{\prime}-\theta^{\prime}\right)+r_{2}^{\prime} \sin \theta^{\prime}\right\} / \sqrt{r^{\prime}{ }_{2} r^{\prime}} \\
& "\left({ }^{\prime \prime}, A y_{7}\right)=\sqrt{y_{0}}\left\{r_{2}^{\prime} \cos \theta^{\prime}-x_{3}^{\prime} \cos \left(3^{\prime}-\theta^{\prime}\right)\right\} / \sqrt{r^{\prime}{ }_{2} r^{\prime}} \\
& \text { " } \quad\left(", \Delta x_{\mathrm{g}}\right)=-\sqrt{\mu_{\mathrm{H}}} r_{2}^{\prime} \sin \theta / \sqrt{r^{\prime}{ }_{2} r_{3}^{\prime}} \\
& \text { " }\left(", \Delta y_{8}\right)=-\sqrt{\mu_{H}} x_{2}^{\prime} \cos \theta 1 / \sqrt{r^{\prime} 2^{r^{\prime}}} \\
& \sqrt{r^{\prime}{ }^{r^{\prime}}}{ }_{4}\left(\Delta 6^{\prime}, \Delta x_{3}\right)=\sqrt{\mu_{0}} r_{3}^{\prime} \sin y^{\prime} / \sqrt{r^{\prime} 3^{r^{2}}} \\
& "\left(", \Delta y_{3}\right)=-\sqrt{\mu_{0}} x_{3}^{\prime} \cos \psi^{\prime} / \sqrt{x^{\prime}{ }_{3} x_{4}} \\
& \text { " (", } \left., \Delta x_{7}\right)=-\sqrt{\mu_{0}} r_{4}^{\prime} \sin \theta^{\prime} / \sqrt{x^{\prime} 3^{r^{\prime}}} \\
& \text { " (", } \left., \Delta y_{7}\right)-\sqrt{\mu_{0}} r_{4}^{\prime} \cos \theta^{\prime} / \sqrt{r^{3} \frac{3}{r}_{4}} \\
& \text { " (" } \left., \Delta x_{8}\right)=\sqrt{\mu_{H}}\left(r^{\prime}{ }_{4} \sin \theta^{\prime}-r_{3}^{\prime} \sin \psi^{\prime}\right\} / \sqrt{x_{3}^{\prime} r_{4}^{\prime}} \\
& \text { " (" } \left., \Delta y_{8}\right)=\sqrt{\mu_{H}}\left(r^{\prime}{ }_{4} \cos \theta^{\prime}+r_{3}^{\prime} \cos \psi^{\prime}\right\} / \sqrt{r^{\prime}{ }_{3} r_{4}^{\prime}} \\
& \sqrt{r_{1} r^{\prime}}\left(\Delta \gamma^{\prime}, \Delta x_{2}\right)=\sqrt{\mu_{c}} r^{\prime}{ }_{4} \sin \left(\gamma^{\prime}-\psi^{\prime}\right) / \sqrt{x_{1} r^{\prime}} \\
& "\left(", \Delta y_{2}\right)=\sqrt{11} c^{\prime}{ }_{4} \cos \left(r^{\prime}-\psi^{\prime}\right) / \sqrt{r_{1} r_{4}^{\prime}} \\
& \text { " (", } \left.\Delta x_{3}\right)=-\sqrt{\mu_{0}}\left\{r_{1} \sin \psi^{*}+r^{\prime} \sin \left(\gamma^{\prime}-\psi^{\prime}\right)\right\} / \sqrt{r_{1} r^{\prime}} \\
& \left." \text { (", }, \Delta y_{3}\right)=\sqrt{\mu_{0}}\left\{r_{1} \cos \psi^{\prime}-r^{\prime}{ }_{4} \cos \left(\gamma^{\prime}-\psi^{\prime}\right)\right\} / \sqrt{r_{1} r^{\prime}} \\
& \text { "(", } \left.\Delta x_{8}\right)=+\sqrt{\mu_{11}} r_{1} \sin \psi / \sqrt{r_{1} r_{4}^{\prime}} \\
& \text { "(", } \left., \Delta y_{8}\right)=-\sqrt{\mu_{H}} x_{1} \cos w^{\prime} / \sqrt{r_{1} r^{\prime}}
\end{aligned}
$$


All other elements are zero

$$
\begin{aligned}
& \mu_{c}=\frac{1}{m_{c}} \\
& \mu_{0}=\frac{1}{m_{0}} \\
& \mu_{H}=\frac{1}{\bar{m}_{H}}
\end{aligned}
$$

where $m_{i}, m_{c}$ and $m_{0}$ are the masses of hydrogen, carbon and oxygen atoms respectively.

17.3 G-matrix in intornal co-ordinates of the inplane vibration of gaseous oxalic acid 2 nd nodol

The G-matrix of the gasecus oxalic acid molecule is here expressed in intemal co-ordinatos in which

$$
\begin{aligned}
& r_{1}=r_{1}^{\prime}, r_{2}=r_{2}^{\prime}, r_{3}=r_{3}^{\prime}, r_{4}=r_{4}^{\prime} \\
& a_{1}=\alpha_{1}^{\prime}, \alpha_{2}=\alpha_{2}^{\prime}, a_{3}=\alpha_{3}^{\prime}, E=\text { a' }^{\prime} \\
& \gamma=\gamma^{2}, 0=0^{\prime}, \hat{\gamma}=\phi^{\prime}, 0=0^{\prime} \\
& \psi=\psi
\end{aligned}
$$

$$
\begin{array}{ll}
G_{1,1}=2 \mu_{c} & G_{1,2}=-\mu_{c} \cos \phi \\
G_{1,3}=-\mu_{c} \cos \phi & G_{1,4}=-\mu_{c} \cos \left(\alpha_{3}-\phi\right) \\
G_{1,5}=-\mu_{c} \cos \left(\alpha_{3}-\phi\right) & G_{1,6}=0 \\
G_{1,7}=0 & G_{1,6}=0 \\
G_{1,0}=0 & G_{1,10}=-\mu_{c} \sin \phi \\
G_{1,11}=-\mu_{c} \sin \left(\alpha_{3}-\phi\right) & G_{1,12}=\left\{\mu_{c} r_{2} \sin \phi+\mu_{c} r_{1} \sin \left(a_{3}-\phi\right)\right\} / \sqrt{r_{1} r_{2}} \\
G_{1,13}=-\mu_{c} \sin \phi & G_{1,14}=-\mu_{c} \sin \left(\alpha_{3}-\phi\right)
\end{array}
$$


$-263-$

$$
\begin{aligned}
& G_{1,15}=\left\{\mu_{c} x_{2} \sin \phi \mu_{c} r_{1} \sin \left(\alpha_{3}-\hat{\phi}\right)\right\} / \sqrt{r_{1} r_{2}} G_{1,10}=\mu_{c} r_{3} \sin \left(\alpha_{3}-\phi\right) / \sqrt{r_{2} r_{3}} \\
& G_{1,17}=0 \\
& G_{1,18} \cdot \mu_{c} r_{4} \sin \phi / \sqrt{r_{1} r_{4}} \\
& G_{1,19}=\mu_{c} r_{3} \sin \left(a_{3}-\xi\right) / \sqrt{r_{2} x_{3}} \\
& G_{1,20}=0 \\
& G_{1,21}=n_{c} x_{4} \sin 6 / \sqrt{r_{1} r_{4}} \\
& \mathrm{G}_{2,1} \cdot \mu_{\mathrm{c}} \cos \phi \\
& \mathrm{G}_{2,3}=0 \\
& G_{2,5}=0 \\
& \mathrm{G}_{2,7}=0 \\
& G_{2,9}=\mu_{0} \cos \gamma
\end{aligned}
$$

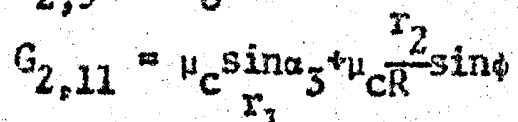

$$
\begin{aligned}
& \mathrm{G}_{2,13}=-\mu_{\mathrm{cR}} \frac{\mathrm{r}_{1}}{\sin \phi} \\
& G_{2,15}=0 \\
& \mathrm{G}_{2,17}=0 \\
& G_{2,19}=0 \\
& G_{2,21}=-\mu_{0} r_{1} \sin \gamma / \sqrt{r_{1} r_{4}} \\
& G_{2,2}=\mu_{c}+\mu_{0} \\
& G_{2,4}-\mu_{c} \cos a_{3} \\
& \mathrm{G}_{2,6}=0 \\
& \mathrm{G}_{2,8}=0 \\
& G_{2,10}=-\mu_{c} \frac{r_{2}}{R} \sin \phi \\
& G_{2,12}=-\mu_{s_{2}} \mathrm{r}_{1} \operatorname{sina}_{3} / \sqrt{r_{1} r_{2}} \\
& G_{2,4}=\mu_{c k} \frac{2}{\sin \phi} \\
& c_{2,16}=-\mu_{c} x_{3} \sin \alpha_{3} / \sqrt{r_{2} r_{3}} \\
& \mathrm{G}_{2,18}=0 \\
& G_{2,20}=\mu_{0} r_{3} \sin / \sqrt{r_{3} r_{4}} \\
& G_{3,1}=-\mu_{c} \cos \phi \\
& G_{3,2}=0 \\
& G_{3,3}=\mu_{c}+\mu_{0} \\
& G_{3,4}=0 \\
& \mathrm{G}_{3,5}=\mu_{c} \cos a_{3} \\
& G_{3,6}=0 \\
& \mathrm{G}_{3,7}=0 \\
& \mathrm{G}_{3,8}=\mu_{0} \cos \gamma \\
& G_{3,9}=0 \\
& G_{3,11}-\mu_{c} \frac{r_{2}}{R_{r_{1}}} \sin \phi \\
& \mathrm{G}_{3,10}=-\mu_{c} \frac{r_{1}}{R} \sin \phi \\
& G_{3,12}=0 \\
& \mathrm{G}_{3,13}=-\mu_{c} \frac{\mathrm{r}_{1}}{\mathrm{R}} \sin \\
& \mathrm{G}_{3,14} \cdot u_{c} \sin \alpha_{3}+\mu_{c} \frac{r_{2}}{R} \sin \phi \\
& G_{3,15}=-\mu_{c} r_{1} \operatorname{sina}_{3} / \sqrt{r_{1} r_{2}} \\
& \mathrm{G}_{3,16}=0 \\
& G_{3,17}=\mu_{0} r_{3} \operatorname{sin\gamma } / \sqrt{r_{3} r_{4}} \\
& \sigma_{3,18}=-\mu_{0} r_{1} \sin \gamma / \sqrt{r_{1} r_{4}}
\end{aligned}
$$




$$
\begin{aligned}
& G_{3,19}=-\mu^{r_{3} \operatorname{sina}_{3} / \sqrt{r_{2} r_{3}}} \quad G_{3,20}=0 \\
& G_{3,21}=0 \\
& G_{4,1}=-\mu_{c} \cos \left(a_{3} m\right) \\
& G_{4,3}=0 \\
& \mathrm{G}_{4,5}=0 \\
& \mathrm{G}_{4,7}=0 \\
& G_{4,9}=0 \\
& G_{4,11}=-\mu_{c_{1}} \frac{r_{2}}{\pi} \sin \left(\alpha_{3}-6\right) \\
& G_{4,13}=\mu_{c} \frac{x_{1}}{h} \sin \left(\alpha_{3}-\theta\right) \\
& G_{4,15}=0 \\
& G_{4,17}=u_{0} x_{4} \sin B / \sqrt{T_{3} x_{4}} \\
& 6_{4,19}=0 \\
& G_{4,21}=-c^{-\mu} c^{x^{\sin _{3}}} / \sqrt{r_{1} r_{4}} \\
& G_{4,2}=\mu_{c} \cos \alpha_{3} \\
& \mathrm{G}_{4,4}=\mu_{\mathrm{c}}+\mu_{0} \\
& G_{4,6}=u_{0} \cos \theta \\
& \mathrm{G}_{4,8}=0 \\
& G_{4,10} \cdot \mu_{c} \sin _{3}{ }^{*} c^{\tau_{1}} \sin \left(a_{3}-\phi\right) \\
& G_{4,12}=-\mu_{c} r_{2} \sin \alpha_{3} / \sqrt{r_{1} r_{2}} \\
& G_{4,14}=-\mu_{c} \frac{2}{R} \sin \left(a_{3}-\phi\right) \\
& G_{4,16}=-\mu_{0} r_{2} \sin \beta / \sqrt{r_{2} r_{3}} \\
& G_{4,18}=0 \\
& \mathrm{G}_{4,20}=0 \\
& G_{5,1}=-\mu_{c} \cos \left(\alpha_{5}-\phi\right) \\
& G_{5,2}=0 \\
& G_{5,3}=\mu_{c} \cos \alpha_{3} \\
& \sigma_{5,4}=0 \\
& \mathrm{G}_{5,5}=\mu_{\mathrm{c}}+\mu_{0} \\
& G_{5,6}=0 \\
& G_{5,7}=\mu_{0} \cos B \\
& G_{5,9}=0 \\
& G_{5,11}=-\mu_{c} \frac{r_{2}}{R} \sin \left(\alpha_{3}-\phi\right) \\
& G_{5,13}-\mu_{c} \sin \alpha_{3}+u_{c} \frac{I_{1}}{R} \sin \left(\alpha_{5}-\phi\right) \\
& \mathrm{G}_{5,8}=0 \\
& G_{5,10}=u_{c} \frac{r_{1}}{R} \sin \left(\alpha_{3}-\phi\right) \\
& \sigma_{5,12}=0 \\
& G_{5,14}=-\mu_{c} \frac{r_{2}}{R} \sin \left(\sigma_{3}-\phi\right) \\
& G_{5,15}=-\mu / c_{2}{ }^{\sin g_{3}} / \sqrt{r_{1} r_{2}} \\
& G_{5,16}=0 \\
& G_{5,17}=0 \\
& G_{5,18}=-\mu_{c} x_{4} \sin \alpha_{3} / \sqrt{r_{1} r_{4}} \\
& G_{5,19}=-\mu_{0} r_{2} \operatorname{sing} / \sqrt{r_{2} r_{3}} \\
& G_{5,21}=0 \\
& G_{5,20}=u_{0} r_{4} \sin \beta / \sqrt{r_{3} r_{4}}
\end{aligned}
$$


$-165-$

$$
\begin{aligned}
& G_{6,1}=0 \\
& 6_{6,3}=0 \\
& G_{6,5}=0 \\
& 6,7-0 \\
& \mathrm{G}_{6,9}=0 \\
& G_{6,11}-\mu_{0} \sin \beta \\
& \mathrm{G}_{6,13}=0 \\
& G_{6,15}=0 \\
& G_{6,17}=-\mu_{4} r_{3} \sin (0+\psi) / \sqrt{x_{3} x_{4}} \\
& G_{6,19}=0 \\
& G_{6,21} \approx 0 \\
& G_{7,1}=0 \\
& \mathrm{G}_{7,3}=0 \\
& G_{7,5}-\mu_{0} \cos \beta \\
& G_{7,7}=u_{0}+\mu_{H} \\
& G_{7,9}=-\mu_{H} \cos (\theta+\beta) \\
& G_{7,11}=0 \\
& 6,7,13=0 \\
& G_{7,15}=-\mu_{0} r_{1} \sin \beta / \sqrt{r_{1} x_{2}} \\
& \sigma_{7,17}=0 \\
& G_{7,19}=-\mu_{0} r_{3} \sin 6 / \sqrt{r_{2} r_{3}} \\
& G_{7,21}=\mu_{11} r_{1} \sin (6+\phi) / \sqrt{r_{1} r_{4}}
\end{aligned}
$$

$$
G_{6,2}=0
$$$$
\mathrm{G}_{6,4}=\mu_{0} \cos \beta
$$$$
6,6=u_{0}+u_{H}
$$$$
G_{6,8}-\mu_{H} \cos (\theta+\varphi)
$$$$
G_{6,10}-0
$$$$
G_{0,12}-\mu_{0} x_{1} \sin \beta / \sqrt{x_{1} r_{2}}
$$$$
6_{6,14}=0
$$$$
\sigma_{6,16}-\mu_{0} r_{3} \sin \beta / \sqrt{r_{2} x_{3}}
$$$$
G_{6,13}=\mu_{H^{x}} \sin (0+\beta) / \sqrt{F_{1} x_{4}}
$$$$
G_{6,20}=0
$$

$$
\begin{aligned}
& G_{7,2}=0 \\
& G_{7,4}=0 \\
& G_{7,6}=0 \\
& G_{7,8}=0 \\
& G_{7,10}=0 \\
& G_{7,12}=0 \\
& G_{7,14}=\mu_{0} \sin B \\
& G_{7,16}=0 \\
& G_{7,18}=0 \\
& G_{7,20}=-\mu_{4} r_{3} \sin (\theta+4) / \sqrt{r_{3} r_{4}}
\end{aligned}
$$



$G_{8,1}=0$
$G_{8,2}=0$
$G_{8,3}=\mu_{0} \cos \gamma$
$G_{S_{8}, 4}=0$
$G_{8,5}=0$
$G_{8,0}=-\mu_{H} \cos (\theta+\theta)$
$\mathrm{G}_{8,7}=0$
$G_{8,8}=\mu_{0}+\mu_{H}$
$\mathrm{G}_{8,9}=0$
$G_{8,10}=0$
$G_{8,11}=0$
$G_{8,12}=0$
$\sigma_{0,13}=\mu_{0} \sin \gamma$
$G_{8,14}=0$
$G_{8,15}=-\mu_{0} r_{2} \sin \gamma / \sqrt{r_{1} r_{2}}$
$G_{3,16}=\mu_{11} r_{2} \sin (\theta+\psi) / \sqrt{r_{2} r_{3}}$
$G_{8,17}=-\mu_{51} r_{4} \sin (\theta+1) / \sqrt{r_{3} r_{4}}$
$6_{8,18}=-u_{0} r_{4} \operatorname{sin\gamma } / \sqrt{r_{1} r_{4}}$
$\mathrm{G}_{8,19}=0$
$G_{8,20}=0$
$G_{\mathcal{E}, 21}=0$
$\mathrm{G}_{9,1}=0$
$G_{9,2}-\mu_{0} \cos \gamma$
$\mathrm{b}_{9,3}=0$
$G_{9,4}=0$
$G_{9,5}=0$
$\mathrm{G}_{9,5}=0$
$G_{9,7}=\mu_{H} \cos (\theta+4)$
$G_{9,8}=0$
$G_{0,9}=\mu_{0}+\mu_{H}$
$G_{0,10}=\mu_{0} \sin \gamma$
$G_{9,11}=0$
$G_{9,12}=-\mu_{0} r_{2} \operatorname{siny} / \sqrt{r_{1} r_{2}}$
$\sigma_{9,13}=0$
$\mathrm{G}_{9,14}=0$
$\mathrm{G}_{\mathrm{Q}, 15}=0$
$G_{9,16}=0$
$\mathrm{G}_{9,17}=0$
$\mathrm{G}_{\mathrm{S}, 18}=0$
$G_{9,19}=\mu_{1}{ }_{2} \sin (\theta+w) / \sqrt{r_{2} r_{3}}$
$G_{9,20}=-\mu_{\mathrm{H}} \mathrm{r}_{4} \sin (\theta+\phi) / \sqrt{r_{3} r_{4}}$
$G_{9,21}=-\mu_{0} r_{4} \sin \gamma / \sqrt{r_{1} r_{4}}$ 


$$
\begin{aligned}
& G_{10,1}=-\mu_{c} \sin \phi \\
& G_{10,3}=-\mu_{c} \frac{r_{1}}{R} \sin \theta \\
& G_{10,5}=-\mu_{c} \frac{T_{2}}{R} \sin \left(\alpha_{3}-6\right) \\
& G_{10,7}=0 \\
& G_{10,9}=\mu_{0} \sin \gamma \\
& G_{10,11}=-2 \mu_{c}\left(r_{1} r_{2} / R^{2}\right)-\mu_{c}\left(r_{2} / R\right) \cos \beta \\
& -\mu_{c}\left(x_{1} / R\right) \cos \left(a_{3}-\phi\right)-\mu_{c} \cos a_{3} \\
& G_{10,13}=2 \mu_{c}\left(r_{1}^{2} / R^{2}\right)+2 \mu_{c}\left(x_{1} / R\right) \cos \phi \\
& G_{10,14}=2 \mu_{c}\left(r_{1} r_{2} / R^{2}\right)-\mu_{c}\left(F_{1} / R\right) \cos \left(a_{5}-\phi\right) \\
& -H_{c}\left(x_{2} / R\right) \cos 4 \\
& G_{10,16}=\left\{u_{c} r_{3} \cos \alpha_{3} n_{c}\left(r_{1} r_{3} / B\right) \cos \left(\alpha_{3}-\phi\right)\right\} \\
& / \sqrt{r_{2} r_{3}} \\
& G_{10,18}=-\mu_{c}\left(\sqrt{r_{1} r_{6}} / R\right) \cos \phi \\
& G_{10,20}=-\mu_{0} x_{3} \cos \beta / \sqrt{r_{3} r_{4}}
\end{aligned}
$$$$
\begin{aligned}
& G_{11,1}=\mu_{c} \sin \left(a_{3}-\phi\right) \\
& G_{11,3}=\mu_{c}\left(r_{2} / R\right) \sin \phi \\
& G_{11,5}=-\mu_{c}\left(r_{2} / R\right) \sin \left(a_{3}-\phi\right) \\
& G_{11,7}=0 \\
& G_{11,9}=0
\end{aligned}
$$$$
\left.G_{11, I 1}=\mu_{c}\left(2 r_{2}^{2} / R^{2}\right) \cdot\left(2 r_{2} / R\right) \cos \left(\alpha_{3}-\phi\right)+1\right)
$$$$
+\mu_{0}
$$$$
G_{11,13}=-2 \mu_{c}\left(r_{1} r_{2} / R^{2}\right)-\mu_{c}\left(r_{1} / R\right) \cos \left(\alpha_{3}-\phi\right)
$$$$
-\mu_{0}\left(r_{2} / R\right) \cos \theta
$$

$$
\begin{aligned}
& G_{10,2}=-\mu_{c} \frac{r_{1}}{R} \sin \phi \\
& G_{10,4}=\mu_{c} \sin \alpha_{3}+\mu_{c R} \frac{r_{1}}{\sin \left(\alpha_{3}-\phi\right)} \\
& G_{10,6}=0 \\
& G_{10,8}=0 \\
& G_{10,10}=\mu_{c}\left(\left(2 r^{2}{ }_{1} / R^{2}\right)+\left(2 r_{1} / N\right) \cos \phi+1\right)+\mu_{0} \\
& G_{10,12}=\left(\mu_{c} r_{1} \cos a_{3}-\mu_{c}\left(r_{1} r_{2} / p\right) \cos \right. \\
& \left.+\mu_{c}\left(r_{1}^{2} / \beta\right) \cos \left(\alpha_{3}-\phi\right)-\mu_{c} x_{2}-\mu_{0} r_{2}\right] \\
& / \sqrt{r_{1} r_{2}} \\
& G_{10,15}-\left(u_{c}\left(r_{1}^{2} / R\right) \cos \left(\omega_{3}-\phi\right)-\mu_{c}\left(r_{1} r_{2} / R\right)\right. \\
& \cos \phi] \sqrt{r_{1} x_{2}} \\
& \mathrm{G}_{10,17}=0 \\
& G_{10,19}=\mu_{c}\left(r_{1} r_{3} / R\right) \cos \left(a_{3}-\phi\right) / \sqrt{r_{2} r_{3}} \\
& G_{10,21}=\left(\mu_{0} x_{1} \cos \gamma-\mu_{0} r_{4}-\mu_{c} r_{4}-\mu_{d}\left(r_{1} r_{4} / I_{3}\right)\right. \\
& \cos \phi\} / \sqrt{r_{1} r_{4}} \\
& G_{11,2} \approx \mu_{c} \sin \alpha_{3}+\mu_{c}\left(r_{2} / R\right) \sin \phi \\
& G_{11,4}=-\mu_{c}\left(r_{2} / R\right) \sin \left(a_{3}-6\right) \\
& \sigma_{11,6} \mu_{0} \sin \beta \\
& \mathrm{G}_{11,3}=0 \\
& G_{11,10}=-2 \mu c\left(r_{1} I_{2} p^{2}\right)-\mu_{c}\left(r_{2} / R\right) \cos \phi \\
& -\mu_{c}\left(r_{1} / R\right) \cos \left(\alpha_{3}-\phi\right)-\mu_{c} \cos \alpha_{3} \\
& 611,12=\left\{\mu_{c}\left(r^{2}{ }_{2} / R\right) \cos 6-\mu_{c}\left(r_{1} x_{2} / R\right)\right. \\
& \cos \left(a_{3}-6\right)+\mu_{c} x_{2} \cos a_{3}-\mu_{c} x_{1} \\
& \left.-\mu_{0} x_{1}\right\} / \sqrt{x_{1} x_{2}}
\end{aligned}
$$




$$
\begin{aligned}
& G_{11,14}=2 \mu_{c}\left(r^{2}{ }_{2} / R^{2}\right)+2 \mu_{c}\left(r_{2} / R\right) \cos \left(\alpha_{3}-\phi\right) \quad \mid G_{11,15}=f_{\mu_{c}}\left(r^{2}{ }_{2} / R\right) \cos \phi-\mu_{c}\left(r_{1} r_{2} / R\right) \\
& G_{11,10}=\left\{\mu_{0} r_{2} \cos \theta-\mu_{0} x_{3}-u_{c}{ }^{r}{ }^{-} \mu_{c}\left(r_{2}{ }^{r} / R\right) \quad \cos \left(a_{3}-\phi\right)\right\} / \sqrt{r_{1} r_{2}} \\
& \left.\cos \left(\alpha_{3}-6\right)\right\} / \sqrt{r_{2} r_{3}} \\
& G_{11,18}=\mu_{c}\left(x_{2} x_{4} / R\right) \cos \phi / \sqrt{x_{1} x_{4}} \\
& G_{11,20}=0 \\
& G_{11,17}=-\mu_{0} r_{4} \cos B / \sqrt{r_{3} r_{4}} \\
& G_{11,19}=-u_{c}\left(\sqrt{r_{2} r_{3}} / R\right) \cos \left(\alpha_{3}-\phi\right) \\
& G_{11,21}=\left\{u_{c} x_{4} \cos \alpha_{3} u_{c}\left(x_{2} x_{4} / R\right) \cos \phi\right\} \\
& \sqrt{r_{1} r_{4}} \\
& G_{12,1}=\left\{\mu_{c} r_{2} \sin \phi \mu_{c} r_{1} \sin \left(\alpha_{3}-\phi\right)\right\} / \sqrt{r_{1} r_{2}} G_{12,2}=-u_{c} r_{1} \sin \alpha_{3} / \sqrt{r_{1} r_{2}} \\
& G_{12,3}=0 \quad G_{2,4}=-\mu_{c} x_{2} \sin \alpha_{3} / \sqrt{r_{1} r_{2}} \\
& G_{12,5}=0 \\
& G_{12,7}=0 \\
& G_{12,9}=-u_{0}{ }_{2} \operatorname{sin\gamma } / \sqrt{x_{1} r_{2}} \\
& G_{12,11}=\left(\mu_{c} r_{2} \cos a_{3}{ }^{-\mu_{c}} c_{1} r_{2} / R\right) \cos \left(c_{3}-\phi\right) \\
& \left.+\mu_{c}\left(r^{2}{ }_{2} / R\right) \cos \phi \quad-\mu_{c} r_{1}-\mu_{0} r_{1}\right\} / \\
& \sqrt{r_{1} x_{2}} \\
& \begin{aligned}
G_{12,13}= & \left\{u_{c}\left(r_{1}^{2} / R\right) \cos \left(\alpha_{3}-\phi\right) H_{c}\left(r_{1} r_{2} / R\right)\right. \\
& \cos \phi / \sqrt{r_{1} r_{2}}
\end{aligned} \\
& \begin{aligned}
G_{12,13}= & \left\{u_{c}\left(r_{1}^{2} / R\right) \cos \left(\alpha_{3}-\phi\right) \mu_{c}\left(r_{1} r_{2} / R\right)\right. \\
& \cos \phi / \sqrt{r_{1} r_{2}}
\end{aligned} \\
& G_{12,0}=-u_{0} r_{1} \sin B \psi_{\sqrt{x_{1} x_{2}}} \\
& G_{12,3}=0 \\
& G_{12,10} * u_{c} r_{1} \cos c_{3}-\mu_{c}\left(r_{1} r_{2} / R\right) \cos \phi \\
& +\mu_{c}\left(r^{2}{ }_{1} / R\right) \cos \left(\alpha_{3}-\phi\right)-\mu_{c} c_{2} \\
& \left.-\mu_{0} x_{2}\right\} / \sqrt{x_{1} r_{2}} \\
& G_{12,12}=\mu_{c}\left(r_{1} / x_{2}+r_{2} / r_{1}-2 \cos \alpha_{3}\right)+ \\
& G_{12,15}=0 \\
& \mu_{0}\left(\left(r_{1} / r_{2}\right)+\left(s_{2} / r_{1}\right)\right) \\
& G_{12,14}=\left\{u_{d}\left(r_{2}^{2} / R\right) \cos \phi-\mu_{d}\left(x_{1} r_{2} / R\right)\right. \\
& \left.\cos \left(a_{3}-4\right)\right) / \sqrt{r_{1} r_{2}} \\
& G_{12,16}=\left(\mu_{c} r_{1} r_{3} / r_{2}\right) \mu_{c} r_{3} \cos \alpha_{3}{ }^{*} \mu_{0}\left(r_{1} r_{3} / r_{2}\right) G_{12,17}=\mu_{0} \sqrt{r_{1} r_{4}} \cos \beta / \sqrt{r_{2} r_{3}} \\
& \left.-\mu_{0} x_{1} \cos \beta\right) / \sqrt{x_{1} x_{3}} \\
& \mathrm{G}_{12,19}=0 \\
& G_{12,18}=0 \\
& G_{12,21}=\left\{\mu_{c}\left(r_{2} r_{4} / r_{1}\right)+\mu_{c} c_{4} \operatorname{cosa}_{3}+\mu_{0}\left(r_{2} r_{4} / r_{1}\right)\right. \\
& G_{12,20}=\mu_{0} \sqrt{r_{2} r_{3}} \cos \gamma / \sqrt{x_{1} r_{4}} \\
& \left.-\mu_{0} r_{2} \cos \gamma\right\} / \sqrt{r_{2} r_{4}}
\end{aligned}
$$




$$
\begin{aligned}
& G_{13,1}=-\mu_{C} \sin \phi \\
& G_{13,3}=-\mu_{c}\left(r_{1} / R\right) \operatorname{sins} \\
& G_{13,5}=u_{c} \sin a_{3}+\mu_{c}\left(r_{2} / R\right) \sin \left(\alpha_{3}-\phi\right) \\
& G_{13,7}=0 \\
& G_{13,9} \approx 0 \\
& G_{13,11}=-2 \mu_{c}\left(r_{1} x_{2} / R^{2}\right)-\mu_{c}\left(r_{1} / R\right) \cos \left(\alpha_{3}-\emptyset\right) \\
& -\mu_{c}\left(x_{2} / R\right) \cos \\
& G_{13,13}=\mu_{c}\left(\left(2 x_{1}{ }^{2} / R^{2}\right)+2\left(r_{1} / R\right) \cos \phi+1\right) * \mu_{0} \\
& G_{13,15}=\left\{\mu_{c} x_{1} \cos \alpha_{3}-\mu_{c}\left(r_{1} r_{2} / R\right) \cos \omega_{-1} \mu_{c} x_{2}\right. \\
& \left.-\mu_{0} r_{2}+\mu_{c}\left(r_{1}^{2} / R\right) \cos \left(\alpha_{3}-\phi\right)\right\} / \sqrt{r_{1} r_{2}} \\
& G_{13,17}=-\mu_{0} r_{3} \cos \gamma / \sqrt{r_{3} r_{4}} \\
& \left.G_{13,19}=\left\{u_{c} c_{2} x_{3} / R\right) \cos \left(a_{3}-\phi\right) * \mu_{1} c_{3} \cos \alpha_{3}\right\} \\
& \sqrt{r_{2}{ }^{3}} \\
& G_{13,21}=-u_{c}\left(\sqrt{r_{1} T_{4}} / R\right) \cos \theta \\
& \mathrm{G}_{14,1} \cdot \mu_{\mathrm{c}} \sin \left(\mathrm{a}_{3}-\hat{\theta}\right) \\
& G_{14,3}=\mu_{c} \sin s_{3} \mu_{c}\left(r_{2} / R\right) \text { sins } \\
& G_{14,5}=-\mu_{c}\left(x_{2} / R\right) \sin \left(\alpha_{3}-\phi\right) \\
& G_{14,7}=\mu_{0} \sin B \\
& G_{14,9}=0 \\
& G_{14,11}=2 \mu_{c}\left(r_{2}{ }^{2} / \mathbb{R}^{2}\right)+2 \mu_{c}\left(r_{2} / R\right) \cos \left(a_{3}-\phi\right) \\
& G_{13,2}=-\mu_{c}\left(k_{1} / R\right) \sin \phi \\
& G_{13,4}=\mu_{c}\left(r_{1} / R\right) \sin \left(\alpha_{3}-\phi\right) \\
& G_{15,6}=0 \\
& G_{13,8}=\mu_{0} \sin \gamma \\
& G_{13,10}=2 \mu_{c}\left(T_{1}^{2} / R^{2}\right)+2 \mu_{c}\left(F_{1} / R\right) \cos \phi \\
& G_{13,12}=\left\{\mu_{c}\left(r_{1}^{2} / R\right) \cos \left(c_{3}-\phi\right)-\mu_{c}\left(r_{1} r_{2} / R\right)\right. \\
& \cos \varphi\} / \sqrt{r_{1} r_{2}} \\
& G_{13,14}=-2 \mu_{c}\left(r_{1} r_{2} / R^{2}\right)-\mu_{c}\left(r_{1} / R\right) \cos \left(\alpha_{3}-\phi\right) \\
& -\mu c\left(x_{2} / R\right) \cos 6-\mu_{c} \cos \alpha_{3} \\
& G_{13,16}=\mu_{c}\left(r_{1} r_{3} / R\right) \cos \left(\alpha_{3}-\uparrow\right) / \sqrt{r_{2} r_{3}} \\
& G_{13,18}=\left(-\mu_{c}\left(r_{1} r_{f} / R\right) \cos \phi-\mu_{c} r_{4}-\mu_{o} r_{4}\right. \\
& \left.\tau \mu_{0} r_{1} \cos \gamma\right\} / \sqrt{r_{1} r_{4}} \\
& G_{13,20}=0 \\
& G_{14,12}=\left\{u_{c}\left(r_{2}^{2} / R\right) \cos \beta-\mu_{c}\left(r_{1} r_{2} / R\right) \cos \left(\alpha_{3}-\phi\right)\right\} \\
& / \sqrt{r_{1} r_{2}} \\
& G_{14,14}=\mu_{c}\left(\left(2 r_{2}{ }^{2} / R^{2}\right)+2\left(\xi_{2} / R\right) \cos \left(\alpha_{3}-\phi\right)+1\right) \\
& +\mu_{0} \\
& G_{14,16}=-\mu_{c}\left(\sqrt{x_{2} x_{3}} / R\right) \cos \left(\alpha_{3}-\phi\right) \\
& G_{14,17}=0 \\
& \begin{aligned}
G_{14,13}= & -2_{11}\left(r_{1} r_{2} / R^{2}\right)-\mu_{c}\left(r_{1} / R\right) \cos \left(a_{3}-\phi\right) \\
& -\mu_{c}\left(r_{2} / R\right) \cos \phi-\mu_{c} \cos \alpha_{3} \\
G_{14,15}= & \left(\mu_{c}\left(r_{2} 2 / R\right) \cos \phi-\mu_{c}\left(r_{1} r_{2} / R\right)\right. \\
& \cos \left(a_{3}-\phi\right) * \mu_{c} r_{2} \cos \alpha_{3}-\mu_{c} r_{1} \\
& \left.-\mu_{0} x_{1}\right) / \sqrt{r_{1} r_{2}}
\end{aligned}
\end{aligned}
$$


$G_{14,18}=\left(u_{c}\left(r_{2} r_{4} / R\right) \cos \phi \mu_{1} c_{4} \cos \alpha_{3}\right\} / \sqrt{r_{1} x_{4}}$

$G_{14,19}=\left[-\mu_{c}\left(r_{2} r_{3} / R\right) \cos \left(c_{3}-\phi\right)-\mu_{c} r_{3}-\mu_{0} r_{3}+\mu_{0} r_{2} \cos s\right\} / \sqrt{r_{2} r_{3}}$

$G_{14,20}=-\mu_{0} r_{4} \cos \beta / \sqrt{r_{3} r_{4}} \quad \mid G_{14,21}=\mu_{c}\left(r_{2} r_{4} / R\right) \cos \phi / \sqrt{x_{1} r_{4}}$

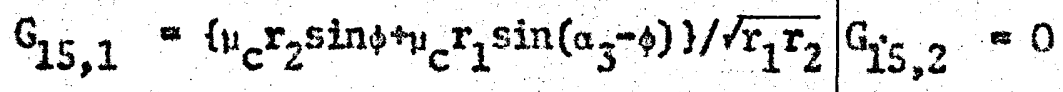

$G_{15,3}=-\mu_{c} r_{1} \sin a_{3} / \sqrt{r_{1} r_{2}}$

$G_{15,5}--\mu_{c} r_{2} \operatorname{sins} s_{3} / \sqrt{r_{1} r_{2}}$

$615,4=0$

$G_{15,7}=-H_{0} I_{1} \sin \beta / \sqrt{r_{1} I_{2}}$

$G_{15,6}=0$

$G_{15,9}=0$

$G_{15,11}=\left\{\mu_{c}\left(r_{2}^{2} / R\right) \cos \phi-\mu_{c}\left(x_{1} r_{2} / R\right) \cos \left(\alpha_{3}\right.\right.$ $-\phi)\} / \sqrt{r_{1} r_{2}}$

$G_{15,8}=-\mu_{0} r_{2} \sin \gamma / \sqrt{r_{1} r_{2}}$

$G_{15,10}=\left(u_{c}\left(x_{1}^{2} / R\right) \cos \left(\alpha_{3}-\beta\right)-\mu_{c}\left(x_{1}{ }^{2} / R\right)\right.$ $\cos \phi\} / \sqrt{r_{1} x_{2}}$

$G_{15,12}=0$

$G_{15,13}=\left\{u_{c} r_{1} \cos \alpha_{3} \nu_{c}\left(r_{1}{ }^{2} / R\right) \cos \left(\omega_{3}-\phi\right)-\mu_{c}\left(r_{2} r_{2} / R\right) \cos \theta-u_{c} r_{2}-u_{0} x_{2}\right\} / \sqrt{r_{1} r_{2}}$

$G_{15,14}=\left\{\mu_{c}\left(r_{2}^{2} / R\right) \cos \phi-\mu_{c}\left(r_{1} I_{2} / R\right) \cos \left(\alpha_{3}-\phi\right)+\mu_{c} c_{2} \cos \alpha_{3}-\mu_{c} r_{1}-\mu_{0} r_{1}\right\} / \sqrt{r_{1} I_{2}}$

$\left.\left.G_{15,15}=\mu_{c}\left(r_{1} / r_{2}\right)+\left(r_{2} / r_{1}\right)-2 \cos \alpha_{3}\right)+u_{0}\left(r_{1} / r_{2}\right)+\left(r_{2} / r_{2}\right)\right)$

$G_{15,16}=0$

$G_{15,17}=\mu_{0} \sqrt{x_{2} r_{3}} \cos \gamma / \sqrt{r_{1} x_{4}}$

$G_{15,18}-\left\{\mu_{c}\left(r_{2} r_{4} / r_{1}\right)-\mu_{c} r_{4} \cos s_{3}+\mu_{0}\left(r_{2} r_{4} / x_{1}\right)-\mu_{0} r_{2} \cos \alpha_{3}\right\} / \sqrt{r_{2} r_{4}}$

$G_{15,19}=\left\{u_{c}\left(r_{1} r_{3} / r_{2}\right)-u_{c} r_{3} \cos \alpha_{3}+u_{0}\left(r_{1} r_{3} / r_{2}\right)-\mu_{0} r_{1} \cos \beta\right\} / \sqrt{r_{1} r_{3}}$

$G_{15,20}=\mu_{0} \sqrt{r_{2} T_{4}} \cos 6 / \sqrt{s_{2} T_{3}}$

$\varepsilon_{15,21}=0$

$G_{16,1}=\mu_{c} x_{3} \sin \left(a_{3}-\phi\right) / \sqrt{x_{2} r_{3}}$

$G_{16,3}-0$

$\mathrm{G}_{16,5}=0$

$G_{16,7}=0$

$\mathrm{G}_{16,2}=-\mu_{c} \mathrm{r}_{3} \sin a_{3} / \sqrt{\mathrm{r}_{2} \mathrm{r}_{3}}$

$G_{16,4}=-\mu_{0} r_{2} \sin \beta / \sqrt{r_{2} r_{3}}$

$c_{16,6}=-\mu_{0} x_{3} \sin B / \sqrt{r_{2} r_{3}}$

$G_{16,9}=0$

$G_{16,8} \otimes \mu_{H^{r}} r_{2} \sin (\theta+\psi) / \sqrt{r_{2} r_{3}}$

$G_{16,10}=\left\{\mu_{c} x_{3} \cos \alpha_{3}+\mu_{c}\left(x_{1} r_{3} / R\right) \cos \left(\alpha_{3}-\phi\right)\right\} / \sqrt{x_{2} x_{3}}$ 


$$
\begin{aligned}
& G_{16,11}=\left\{w_{0} r_{2} \cos -n_{0} r_{3}-H_{c} r_{3}-\mu_{c}\left(r_{2} r_{3} / R\right) \cos \left(\alpha_{3}-\phi\right)\right\} / \sqrt{r_{2} r_{3}} \\
& G_{16,12}=\left\{\mu_{c}\left(r_{1} r_{3} / r_{2}\right)-u_{c} r_{3} \cos a_{3}+\mu_{0}\left(r_{1} r_{3} / r_{2}\right)-\mu_{0} r_{1} \cos \beta\right\} / \sqrt{r_{1} r_{3}} \\
& G_{16,13}=\mu_{c}\left(r_{1} r_{3} / R\right) \cos \left(\alpha_{3}-\phi\right) / \sqrt{r_{2} r_{3}} \quad G_{16,14}=-u_{c} \sqrt{r_{2} r_{3}} / R \cos \left(\alpha_{3}-\phi\right) \\
& 0_{16,15}=0 \\
& \mathrm{G}_{2}, 16=\mu_{c}\left(r_{3} / r_{2}\right)+\mu_{1}\left(r_{2} / r_{3}\right)+\mu_{0}\left(\left(r_{2} / r_{3}\right)+\left(r_{3} / r_{2}\right)-2 \cos \beta\right) \\
& G_{16,17}=\left\{-\mu_{0}\left(r_{2} r_{4} / r_{3}\right)+\mu_{0} r_{4} \cos 6-\mu_{4}\left(r_{2} r_{4} / r_{3}\right)-\mu_{11} r_{2} \cos (\theta+b)\right\} / \sqrt{r_{2} r_{4}} \\
& G_{10,18}=\nu_{H} \sqrt{r_{1 r_{2}}} \cos (\theta+\psi) / \sqrt{r_{3} x_{4}} \quad G_{16,10}=0 \\
& \mathrm{G}_{16,20}=0 \\
& G_{17,1}=0 \\
& G_{17,3}=\mu_{0} x_{3} \sin / \sqrt{r_{3} x_{4}} \\
& G_{17,5}=0 \\
& G_{17,7}=0 \\
& G_{17,9}=0 \\
& G_{17,11}=-\mu_{0} r_{4} \cos \beta / \sqrt{r_{3} r_{4}} \\
& G_{17,13}=-\mu_{0} x_{3} \cos \gamma / \sqrt{r_{3} x_{4}} \\
& G_{17,25}=\mu_{0}{\sqrt{r_{2} r_{3}}} \cos \gamma / \sqrt{r_{1} r_{4}} \\
& \sigma_{10,21}=-\mu_{c} \sqrt{r_{3} r_{4}} \cos \alpha_{3} / \sqrt{r_{1} r_{2}} \\
& { }_{17,2}=0 \\
& G_{17,4}=\mu_{0} r_{4} \sin \beta / \sqrt{r_{3} r_{4}} \\
& G_{17,6}=-\mu_{4} r_{3} \sin (\theta+1) / \sqrt{r_{3} r_{4}} \\
& G_{17,8}=-u_{1} r_{4} \sin (\theta+1) / \sqrt{r_{3} r_{4}} \\
& G_{17,10}=0 \\
& G_{17,12}=\mu_{0} \sqrt{r_{1} x_{4}} \cos 6 / \sqrt{r_{2} r_{3}} \\
& G_{17,14}=0 \\
& G_{17,16}=\left\{-\mu_{0}\left(r_{2} r_{4} / r_{3}\right)+\mu_{0} r_{4} \cos \beta-\mu_{1}\left(r_{2} r_{4} / r_{3}\right)-\mu_{11} r_{2} \cos (\theta+\psi)\right\} / \sqrt{r_{2} r_{4}} \\
& G_{17,17}=\mu_{0}\left(\left(r_{3} / r_{4}\right)+\left(r_{4} / r_{3}\right)\right)+\mu_{15}\left(\left(r_{3} / r_{4}\right)+\left(r_{4} / r_{3}\right)+2 \cos (B+\beta)\right) \\
& G_{17,18}=\left\{-\mu_{1}\left(r_{1} r_{3} / r_{4}\right)-\mu_{11} r_{1} \cos (\xi+\psi)-\mu_{0}\left(r_{1} r_{3} / r_{4}\right)+\mu_{0} r_{3} \cos \right\} / \sqrt{r_{1} r_{3}} \\
& G_{17,19}=0 \\
& G_{17,21}=0 \\
& G_{17,20}=0 \\
& G_{13,1}=\mu_{c^{x_{4}}} \sin \phi / \sqrt{r_{1} r_{4}} \\
& G_{18,3}=-\mu_{0} r_{1} \sin \gamma / \sqrt{I_{1} I_{4}} \\
& G_{18,5}=-\mu_{c} r_{4} \operatorname{sina}_{3} / \sqrt{x_{1} r_{4}} \\
& G_{18,2}=0 \\
& G_{18,4}=0 \\
& \left.G_{18,6}=u_{11} r_{1} \sin (\theta+1)\right) / \sqrt{r_{1} r_{4}}
\end{aligned}
$$




$$
\begin{aligned}
& G_{18,7}=0 \\
& G_{18,9}=0 \\
& G_{18,11}=\mu_{c}\left(r_{2} v_{4} / R\right) \cos \phi / \sqrt{x_{1} r_{4}} \\
& G_{18,8}=-\mu_{0} x_{4} \sin \gamma / \sqrt{r_{1} r_{4}} \\
& r_{18,10}=-u^{\left(\sqrt{r_{1} r_{4}} / R\right) \cos } \\
& G_{18,12}=0 \\
& G_{12,15}=\left\{-\mu_{0}\left(r_{1} r_{4} / R\right) \cos ^{4}-u_{c} r_{4}-u_{0} x_{4}+\mu_{0} r_{1} \cos \gamma\right\} / \sqrt{r_{1} x_{4}} \\
& \left.G_{18,14}=u_{c}\left(r_{2} r_{4} / R\right) \cos \phi+\mu_{c} r_{4} \cos a_{3}\right\} / \sqrt{r_{1} r_{4}} \\
& \mathrm{G}_{18,15}=\left\{u_{c}\left(r_{2} r_{4} / r_{1}\right)-u_{c} r_{4} \cos a_{3}-u_{0} r_{2} \cos \alpha_{3}+\mu_{0}\left(r_{2} r_{4} / r_{1}\right)\right\} / \sqrt{r_{2} r_{4}} \\
& G_{18,16}=\mu_{i 1} \sqrt{r_{1} x_{2}} \cos (\theta+\phi) / \sqrt{r_{3} r_{4}} \\
& G_{18,17}=\left\{-\mu_{11}\left(r_{1} r_{3} / x_{4}\right)-\mu_{11} r_{1} \cos (\theta+4)-\mu_{0}\left(r_{1} r_{3} / r_{4}\right)+\mu_{0} r_{3} \cos \right\} / \sqrt{r_{1} r_{3}} \\
& G_{18,18}=u_{c}\left(r_{4} / r_{1}\right)+u_{15}\left(r_{1} / r_{4}\right)+u_{0}\left(\left(r_{1} / r_{8}\right)+\left(r_{4} / r_{1}\right)-2 \cos \gamma\right)
\end{aligned}
$$

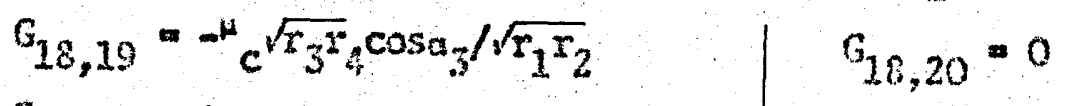

$$
\begin{aligned}
& G_{18,21}=0 \\
& G_{19,1}=\mu_{c} x_{3} \sin \left(a_{3}-\phi\right) / \sqrt{x_{2} r_{3}} \\
& G_{19,3}=-c^{x_{3} \sin \alpha_{3} / \sqrt{x_{2} x_{3}}} \\
& G_{19,5}=-\mu_{0} T_{2} \operatorname{sin\beta } / \sqrt{r_{2} r_{3}} \\
& G_{19,7}=-\mu_{0} r_{3} \sin \beta / \sqrt{r_{2} r_{3}} \\
& G_{19,9}=v_{11} x_{2} \sin (\theta+4) / \sqrt{x_{2} r_{3}} \\
& G_{19,11}=-\mu\left(\sqrt{r_{2} r_{3}} / R\right) \cos \left(\alpha_{3}-\phi\right) \\
& G_{29,2}=0 \\
& \mathrm{G}_{19,4}=0 \\
& G_{19,6}=0 \\
& c_{19,8}=0 \\
& G_{19,10}=\mu_{c}\left(r_{1} r_{3} / R\right) \cos \left(c_{3}-\phi\right) / \sqrt{r_{2} r_{3}} \\
& G_{10,12}=0 \\
& G_{19,13}=\left\{\mu_{c}\left(r_{1} I_{3} / R\right) \cos \left(\alpha_{3}-\phi\right)+\mu_{c} x_{3} \cos a_{3}\right\} / \sqrt{r_{2} x_{3}} \\
& G_{10,14}=\left(-u_{c}\left(r_{2} r_{3} / R\right) \cos \left(\alpha_{3}-\phi\right)-u_{c} r_{3}-u_{0} r_{3}+u_{0} r_{2} \cos \beta\right) / \sqrt{r_{2} r_{3}} \\
& G_{1 D_{1} 15}=\left\{\mu_{c}\left(r_{1} r_{3} / r_{2}\right)-\mu_{c} r_{3} \cos \alpha_{3}+\mu_{0}\left(r_{1} r_{3} / r_{2}\right)-\mu_{0} r_{1} \cos \beta\right\} / \sqrt{r_{1} r_{3}} \\
& G_{19,16}=0 \\
& G_{19,18}=-\mu_{c} \sqrt{r_{3} T_{4}} \cos \alpha_{3} / \sqrt{r_{1} T_{2}} \\
& \mathrm{G}_{19,17}=0 \\
& \left.G_{19,19}=\mu_{c}\left(r_{3} / r_{2}\right)+\mu_{12}\left(r_{2} / r_{3}\right)+u_{0}\left(r_{2} / r_{3}\right)+\left(r_{3} / r_{2}\right)-2 \cos \beta\right) \\
& G_{19,20}=\left\{-\mu_{0}\left(r_{2} r_{4} / r_{3}\right) \div \mu_{0} r_{4} \cos \beta-\mu_{4}\left(r_{2} r_{4} / r_{3}\right)-\mu_{1} r_{2} \cos (\theta+i)\right\} / \sqrt{r_{2} r_{4}} \\
& G_{19,21}=\mu_{11} \sqrt{r_{1} r_{2}} \cos (\theta+v) / \sqrt{r_{3} x_{4}}
\end{aligned}
$$




$$
\begin{aligned}
& \mathrm{G}_{20,1}=0 \\
& G_{20,3}=0 \\
& \mathrm{G}_{20,5}=\mu_{0} \mathrm{r}_{4} \sin \beta / \sqrt{r_{3} \mathrm{r}_{4}} \\
& G_{20,7}=-\mu_{41} r_{3} \sin (\theta+b) / \sqrt{r_{3} r_{4}} \\
& G_{20,9}=-\mu_{H 1} r_{4} \sin (\theta+\psi) / \sqrt{r_{3} r_{4}} \\
& \mathrm{G}_{20,31}=0 \\
& G_{20,13}=0 \\
& G_{20,15}=\mu_{0} \sqrt{r_{1} r_{4}} \cos B / \sqrt{r_{2} T_{3}} \\
& \mathrm{G}_{20,17}=0 \\
& G_{20,2}=\mu_{0} r_{3} \sin \gamma / \sqrt{r_{3} r_{4}} \\
& \mathrm{G}_{20,4}=0 \\
& G_{20,6}=0 \\
& G_{20,8}=0 \\
& G_{20,10}-\mu_{0} r_{3} \cos 6 / \sqrt{r_{3} r_{4}} \\
& G_{20,12}=\mu_{0} \sqrt{r_{2} r_{3}} \cos \gamma / \sqrt{r_{1} r_{4}} \\
& G_{20,14}=-\mu_{0} r_{4} \cos \beta / \sqrt{r_{3} r_{4}} \\
& \mathrm{~b}_{20,16}=\mathrm{o} \\
& G_{20,18}=0 \\
& G_{20,19}=\left\{-u_{0}\left(r_{2} r_{4} / r_{3}\right)+\mu_{0} r_{4} \cos \beta-\mu_{1}\left(r_{2} r_{4} / r_{3}\right)-\mu_{1} r_{2} \cos (9+4)\right\} / \sqrt{r_{2} r_{4}} \\
& \left.G_{20,20}=\mu_{0}\left(r_{3} / r_{4}\right)+\left(r_{4} / r_{3}\right)+\mu_{H}\left(r_{3} / r_{4}\right)+\left(r_{4} / r_{3}\right)+2 \cos (0+0)\right) \\
& G_{20,21}=\left(-\mu_{0}\left(r_{1} I_{3} / r_{4}\right)+\mu_{0} r_{3} \cos \gamma-\mu_{1}\left(r_{1} r_{3} / r_{4}\right)-\mu_{H} r_{1} \cos (0+\phi)\right\} / \sqrt{r_{1} r_{3}} \\
& G_{21,1}=\mu_{c} x_{4} \sin \phi / \sqrt{r_{1} r_{4}} \\
& G_{21,3}=0 \\
& \mathrm{G}_{21,5}=0 \\
& G_{21,7}=\mu_{H_{1}} \sin (\theta+4) / \sqrt{r_{1} r_{4}} \\
& G_{21,9}=-u_{0} x_{4} \sin \gamma / \sqrt{r_{1} r_{4}} \\
& \mathrm{G}_{21,2}=-\mu_{0} \mathrm{r}_{1} \sin \gamma / \sqrt{\mathrm{r}_{1} \mathrm{r}_{4}} \\
& G_{21,4}=-\mu_{c} r_{4} \operatorname{sina}_{3} / \sqrt{r_{1} r_{4}} \\
& \mathrm{G}_{21,6}=0 \\
& G_{21,8}=0 \\
& G_{21,10}=\left\{\mu_{0} r_{1} \cos \gamma-\mu_{0} x_{4}-\mu_{c} x_{4}-\mu_{c}\left(r_{1} x_{4} / R\right) \cos \phi\right\} / \sqrt{r_{1} r_{4}} \\
& G_{21,11}=\left\{\mu_{c} x_{4} \cos \alpha_{3}+\mu_{c}\left(r_{2} x_{4} / R\right) \cos \phi 1 / \sqrt{r_{1} x_{4}}\right. \\
& G_{21,12}=\left\{u_{c}\left(r_{2} r_{4} / r_{1}\right)-\mu_{c} r_{4} \cos a_{3}+\mu_{0}\left(r_{2} r_{4} / r_{1}\right)-u_{0} r_{2} \cos \gamma / \sqrt{r_{2} r_{4}}\right. \\
& G_{21,13}=-\mu_{c}\left(\sqrt{r_{1} r_{4}} / R\right) \cos \phi \\
& G_{21,15}=0 \\
& \mathrm{G}_{21,27}=0 \\
& G_{21,19}=\mu_{31} \sqrt{r_{1} r_{2}} \cos (\theta+\psi) / \sqrt{r_{3} r_{4}} \\
& G_{21,14}=\mu_{C}\left(r_{2} r_{4} / R\right) \cos \phi / \sqrt{r_{1} r_{4}} \\
& G_{21,10}=-\mu_{c} \sqrt{r_{3} x_{4}} \cos \alpha_{3} / \sqrt{r_{1} r_{2}} \\
& \mathrm{G}_{21,18}=0 \\
& G_{21,20}=\left\{-\mu_{0}\left(r_{1} r_{3} / r_{4}\right)+\mu_{0} r_{3} \cos \gamma-\mu_{13}\left(r_{1} r_{3} / r_{4}\right)-\mu_{17} r_{1} \cos \left(\theta+\mu_{0}\right)\right\} / \sqrt{r_{1} r_{3}} \\
& G_{21,21}=\mu_{c}\left(r_{4} / r_{1}\right)+\mu_{H}\left(r_{1} / r_{4}\right) * u_{0}\left(\left(r_{1} / r_{4}\right) *\left(r_{4} / r_{1}\right)-2 \cos \gamma\right)
\end{aligned}
$$


17.4 Gymotry co-ordinates of the inplane vibuation of gaseous

oxalic acid 2nd nozel

The trmafomation table of the intemal co-ordinates of gaseous oxalic acid 2 nd nodol is given below.

Table 14

\begin{tabular}{|c|c|c|c|c|}
\hline $\mathrm{c}_{2 \mathrm{~h}}$ & $E$ & $c_{2}$ & $i$ & $\sigma_{h}$ \\
\hline$\Delta R$ & $\Delta R$ & $\Delta R$ & $\Delta R$ & $\Delta \mathbb{R}$ \\
\hline$\Delta r_{1}$ & $\Delta r_{1}$ & $\Delta r^{\prime}{ }_{1}$ & $\Delta r_{1}^{\prime}$ & $\Delta r_{1}$ \\
\hline$\Delta r^{\prime}{ }_{1}$ & $\Delta r^{\prime}{ }_{2}$ & $\Delta r_{1}$ & $\Delta \tau_{1}$ & $\Delta T_{1}^{\prime}$ \\
\hline$\Delta x_{2}$ & $\Delta r_{2}$ & $\Delta x_{2}^{\prime}$ & $\Delta r_{2}^{\prime}$ & $\Delta I_{2}$ \\
\hline$\Delta r_{2}^{\prime}$ & $\Delta x_{2}^{\prime}$ & $\Delta r_{2}$ & $\Delta x_{2}$ & $\Delta \mathrm{r}_{2}^{\prime}$ \\
\hline$\Delta r_{3}$ & $\Delta r_{3}$ & $\Delta r_{3}^{\prime}$ & $\Delta r_{3}^{\prime}$ & $\Delta x_{3}$ \\
\hline$\Delta x^{\prime}{ }_{3}$ & $\Delta r_{3}^{\prime}$ & $\Delta r_{3}$ & $\Delta r_{3}$ & $\Delta r^{\prime}{ }_{3}$ \\
\hline$\Delta x_{A}$ & $\mathrm{Ar}_{4}$ & $\Delta r_{4}^{\prime}$ & $\Delta \mathbf{x}_{4}^{\prime}$ & $\Delta r_{4}$ \\
\hline$\Delta \mathbf{r}_{4}{ }_{4}$ & $\Delta r^{\prime}{ }_{4}$ & $\Delta x_{\Delta}$ & $\Delta r_{4}$ & $\Delta r^{\prime}{ }_{A}$ \\
\hline$\Delta \Delta_{1}$ & $\Delta a_{1}$ & $\Delta Q^{\prime}{ }_{1}$ & $\Delta a^{\prime}{ }_{1}$ & $\Delta a_{1}$ \\
\hline$\Delta \alpha_{2}$ & $\Delta a_{2}$ & $\Delta \alpha_{2}^{\prime}$ & $\Delta a_{2}^{\prime}$ & $\Delta \alpha_{2}$ \\
\hline$\Delta \alpha_{3}$ & $\Delta a_{3}$ & $\Delta x^{\prime}{ }_{3}$ & $\Delta \alpha_{3}^{\prime}$ & $\Delta \alpha_{3}$ \\
\hline$\Delta \otimes^{\prime}{ }_{1}$ & $\Delta a_{1}^{\prime}$ & $\Delta o_{1}$ & $\Delta a_{1}$ & $\Delta \alpha_{1}^{\prime}$ \\
\hline$\Delta \alpha_{2}^{\prime}$ & $\Delta \alpha^{\prime}{ }_{2}$ & $\Delta a_{2}$ & $\Delta \alpha_{2}$ & $\Delta \alpha^{\prime}{ }_{2}$ \\
\hline$\Delta \Delta_{3}^{\prime}$ & $\Delta c^{\prime}{ }_{3}$ & $\Delta a_{3}$ & $\Delta c_{3}$ & $\Delta \alpha^{\prime}{ }_{3}$ \\
\hline$\Delta B$ & $\Delta \beta$ & $\Delta \beta^{\prime}$ & $\Delta \beta^{\prime}$ & $\Delta B$ \\
\hline$\Delta 6$ & $\Delta \delta$ & $\Delta \delta^{\prime}$ & $\Delta 0^{\prime}$ & $\Delta \delta$ \\
\hline$\Delta r$ & $\Delta \gamma$ & $\Delta \gamma^{\prime}$ & $\Delta \gamma^{\prime}$ & $\Delta y$ \\
\hline$\Delta B^{\prime}$ & $\Delta B^{\prime}$ & $\Delta B$ & $A B$ & $\Delta \beta^{\prime}$ \\
\hline$\Delta 0^{\prime}$ & $\Delta \delta^{\prime}$ & $\Delta S$ & $\Delta S$ & $\Delta B^{\prime}$ \\
\hline$\Delta \gamma^{*}$ & $\Delta Y^{\prime}$ & $\Delta \gamma$ & $\Delta \gamma$ & $\Delta \gamma^{\prime}$ \\
\hline
\end{tabular}


The sane process as in 15.4 applies and the resultant nomalised symetry co-ordinates for gasoous oxalic acid 2 nd model are

As set

$$
\begin{aligned}
& S_{1}=\Delta R \quad \text { symetric C-C stretch } \\
& S_{2}=\frac{1}{\sqrt{2}}\left(\Delta r_{1}+\Delta r^{\prime}\right) \quad \text { synetric C=0 stretch } \\
& s_{3}=\frac{1}{\sqrt{2}}\left(\Delta r_{2}+\Delta r_{2}^{\prime}\right) \quad \text { symetric } C-0 \text { stretch } \\
& s_{4}=\frac{1}{\sqrt{2}}\left(\Delta r_{3}+\Delta r^{\prime}{ }_{3}\right) \quad \text { symatric o- il stretch } \\
& s_{\mathrm{g}}=\frac{1}{\sqrt{2}}\left(\Delta r_{4}+\Delta x_{4}\right) \quad \text { symetric } 0 . . . \text { H stretch } \\
& s_{6}=\frac{1}{\sqrt{3}}\left(\Delta a{ }_{3}+\Delta a^{\prime}{ }_{3}\right)-\frac{1}{2 \sqrt{3}}\left(\Delta a_{1}+\Delta a_{2}+\Delta a^{\prime}{ }_{1}+\Delta a_{2}^{\prime}\right) \\
& \text { symatric }(-C \leqslant 9) \text { angle deformation } \\
& s_{7}=\frac{1}{2 \sqrt{3}}\left(\Delta \beta+\Delta \gamma^{+}+\Delta \varepsilon^{\prime}+\Delta \gamma^{\prime}\right)-\frac{1}{\sqrt{3}}\left(\Delta Q+\Delta \delta^{\prime}\right) \text { sjmatric }(0-11 . .0) \text { angle bending }
\end{aligned}
$$

Bu set

$$
\begin{aligned}
& S_{S}=\frac{1}{\sqrt{2}}\left(\Delta x_{1}-\Delta x^{\prime}{ }_{1}\right) \quad \text { unti-symatric } e=0 \text { stretch } \\
& S_{9}=\frac{1}{\sqrt{2}}\left(\Delta r_{2}-\Delta r_{2}^{\prime}\right) \quad \text { anti-symatric } c-0 \text { stretch } \\
& s_{10}=\frac{1}{\sqrt{2}}\left(\Delta r_{3}-\Delta x_{3}^{\prime}\right) \quad \text { anti-symotric } 0 \text {-H stretch } \\
& s_{11}=\frac{1}{\sqrt{2}}\left(\Delta r_{4}+\Delta H^{\prime}\right) \quad \text { anti-symetric } 0 . . \text { H stretch } \\
& S_{12}=\frac{1}{\sqrt{3}}\left(\Delta a_{3}-4 a_{3}^{\prime}\right)-\frac{1}{2 \sqrt{3}}\left(\Delta a_{1}+\Delta a_{2}-\Delta a_{1}^{\prime}-\Delta a_{2}^{\prime}\right) \\
& \text { anti-symatric }(-\mathrm{C} \leqslant 0) \text { angle } \\
& \text { deformation } \\
& S_{13}=\frac{1}{2 \sqrt{3}}\left(\Delta B+\Delta \gamma-\Delta B^{\prime}-\Delta \gamma^{\prime}\right)-\frac{1}{\sqrt{3}}\left(\Delta \delta-\Delta \delta^{\prime}\right) \\
& \text { anti-symotric }(0-11 . . .0) \text { angle } \\
& \text { bending }
\end{aligned}
$$

From the nomaized symetry co-orininates the U-matrix in Table 15 is obtained. 


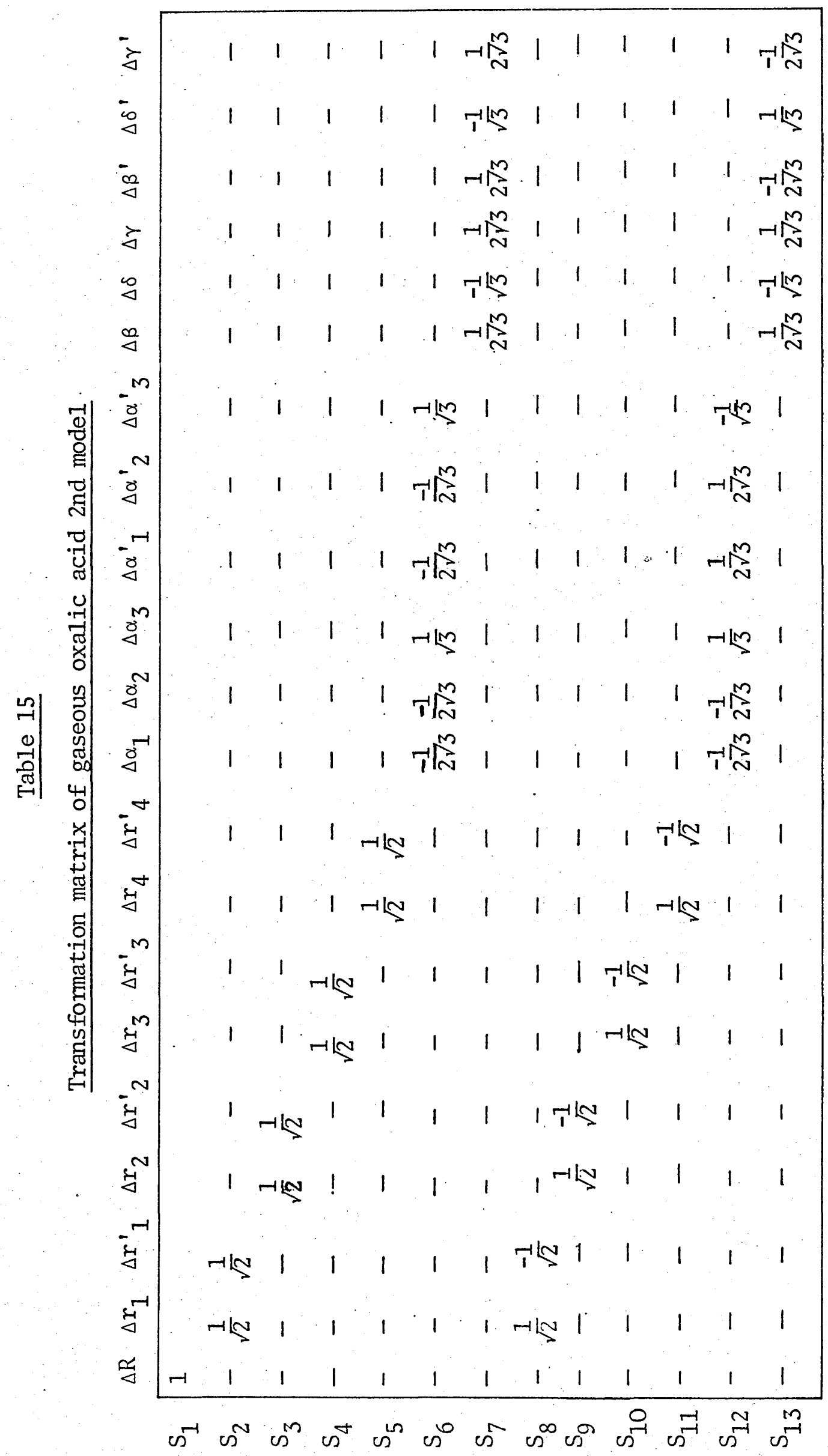


17.5 Numorical values of G-matrix of gaseous oxalic acid 2nd model in symetrycco-ordinates

$$
\begin{array}{ll}
G(1,1)=0.166657 & G(1,2)=-0.054359 \\
G(1,3)=-0.043957 & G(1,4)=0 \\
G(1,5)=0 & G(1,6)=0.254615 \\
G(1,7)=0.091953 & \\
G(1,8)=G(1,9)=G(1,10)=G(1,11)=G(1,12)=G(1,13)=0 \\
G(2,1)=-0.064359 & G(2,2)=0.145333 \\
G(2,3)=-0.047798 & G(2,4)=0 \\
G(2,5)=0.009076 & G(2,6)=-0.035632 \\
G(2,7)=-0.080574 & \\
G(2,8)=G(2,9)=G(2,10)=G(2,11)=G(2,12)=G(2,13)=0 \\
G(3,1)=-0.043957 & G(3,2)=-0.047793 \\
G(3,3)=0.145335 & G(3,4)=-0.015543 \\
G(3,5)=0 & G(3,6)=-0.031205 \\
G(3,7)=-0.13261 & G(4,6)=-0.071662 \\
G(3,8)=G(3,9)=G(3,10)=G(3,11)=G(3,12)=G(3,13)=0 \\
G(4,1)=0 \\
G(4,3)=-0.015543 \\
G(4,5)=-0.484054
\end{array}
$$




$$
\begin{aligned}
& G(5,1)=0 \quad G(5,2)=0.009076 \\
& G(5,3)=0 \quad G(5,4)=-0.484054 \\
& G(5,5)=1.062513 \quad G(5,6)=-0.078403 \\
& G(5,7)=1.361267 \\
& G(5,8)=G(5,9)=G(5,10)=G(5,13)=G(5,12)=G(5,15)=0 \\
& G(0,1)=0.254615 \quad G(6,2)=-0.085632 \\
& G(6,3)=-0.001200 \quad G(0,4)=-0.071602 \\
& G(6,5)=-0.078403 \quad G(6,0)=0.579780 \\
& G(6,7)=0.230543 \\
& G(6,0)=G(0,9)=G(6,10)=G(6,11)=G(6,12)=G(6,13)=0 \\
& G(7,1)=0.091953 \quad G(7,2)=-0.080574 \\
& G(7,3)=-0.132610 \quad G(7,4)=0.768012 \\
& G(7,5)=1.361267 \quad G(7,0)=0.230543 \\
& G(7,7)=5.042583 \\
& G(7,8)=G(7,9)=G(7,10)=G(7,11)=G(7,12)=G(7,13)=0 \\
& G(8,1)=G(8,2)=G(8,3)=G(8,4)=G(8,5)=G(8,6)=G(8,7)=0 \\
& G(8,8)=0.145333 \quad G(8,9)=-0.047708 \\
& G(8,10)=0 \quad G(8,11)=-0.009076 \\
& G(8,12)=-0.080508 \quad G(8,13)=0.051076 \\
& G(9,1)=G(9,2)=G(9,3)=G(9,4)=G(9,5)=G(9,6)=G(9,7)=0 \\
& \mathrm{G}(0,8)=-0.047798 \quad \mathrm{G}(9,9)=0.145835 \\
& G(9,10)=-0.015543 \quad G(9,11)=0 \\
& G(9,12)=-0.036549 \quad G(0,13)=-0.050272
\end{aligned}
$$




$$
\begin{array}{ll}
G(10,1)=G(10,2)=G(10,3)=G(10,4)=G(10,5)=G(10,6)=G(10,7)=0 \\
G(10,8)=0 & G(10,9)=-0.015543 \\
G(10,10)=1.052499 & G(10,11)=-0.484054 \\
G(10,12)=-0.071662 & G(10,13)=0.768012 \\
G(11,1)=G(11,2)=G(11,3)=G(11,4)=G(11,5)=G(11,6)=G(11,7)=0 \\
G(11,3)=-0.039076 & G(11,9)=0 \\
G(11,10)=-0.484054 & G(11,11)=1.062513 \\
G(11,12)=0.078403 & G(11,13)=1.361267 \\
G(12,1)=G(12,2)=G(12,3)=G(12,4)=G(12,5)=G(12,6)=G(12,7)=0 \\
G(12,8)=-0.080803 & G(12,9)=-0.086549 \\
G(12,10)=-0.071662 & G(12,11)=0.078403 \\
G(12,12)=0.582270 & G(12,13)=-0.003687 \\
G(13,1)=G(13,2)=G(13,3)=G(13,4)=G(13,5)=G(13,6)=G(13,7)=0 \\
G(13,8)=0.031076 \quad G(13,9)=-0.050272 \\
G(13,10)=0.768012 & G(13,11)=1.361267 \\
G(13,12)=-0.003687 & G(13,13)=5.005856 \\
&
\end{array}
$$




\section{CHAETER 18}

CONSTRUCTION OF TIE E-MATRIX OF THE IALLANE VIBRATIONS

OF GASEOUS OXALIC ACII 2ND MOUIL

18.1 Urey-Bradley force field of gaseous oxalic acid 2 nd model

The U.B.F.F. for the inplane vibrations of gaseous oxalic acid $2 \mathrm{nd}$ model was constructed using $\mathrm{K}$ as the stretching force constant, $H$ as the bending force constant, and $F$ the repuisive force constant between non-bonded atons.

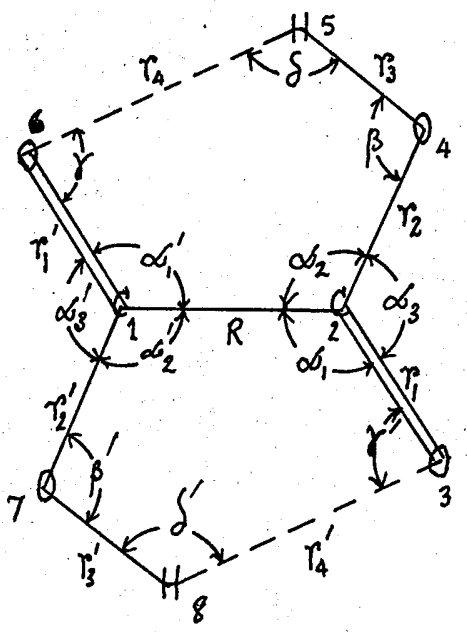

Fin. 22

The potential energy of the oxalic acid can be expressed as

$$
\begin{aligned}
& V=\frac{1}{3} \mathrm{~K}_{C C}(\Delta R)^{2}+K^{\prime} C_{C} R(\Delta R) \\
& \text { - }{ }_{2}^{1} K_{C a O}\left(\Delta r_{1}\right)^{2}+K_{C=0}^{\prime} r_{1}\left(\Delta r_{1}\right) \\
& +\frac{1}{2} K_{C=0}\left(\Delta r^{\prime}{ }_{1}\right)^{2}+K^{\prime}{ }_{C=0} r^{\prime}{ }_{1}\left(\Delta r^{\prime}{ }_{1}\right) \\
& +\frac{1}{2} \mathrm{~A}_{\mathrm{C}-\mathrm{O}}\left(\Delta \mathrm{r}_{2}\right)^{2}+\mathrm{K}_{\mathrm{C}-\mathrm{O}}^{\prime} \mathrm{r}_{2}\left(\Delta \mathrm{r}_{2}\right) \\
& +1 k_{C-0}\left(\Delta t_{2}^{\prime}\right)^{2}+K_{C-0}^{\prime} r_{2}^{\prime}\left(\Delta r_{2}^{\prime}\right) \\
& +\frac{1}{2} K_{0-H_{1}}\left(\Delta r_{3}\right)^{2}+K_{0-11} r_{3}\left(\Delta r_{3}\right)
\end{aligned}
$$


$+1 \mathrm{~K}_{\mathrm{O}-\mathrm{H1}}\left(\Delta \mathrm{r}_{3}^{\prime}\right)^{2}+\mathrm{K}_{\mathrm{O}-\mathrm{H}} \mathrm{r}^{\prime}{ }_{3}\left(\Delta \mathrm{r}_{3}^{\prime}{ }_{3}\right)$

$+\frac{1}{1} r_{0 . .11}\left(\Delta r_{4}\right)^{2}+K_{0 . . H} r_{4}\left(\Delta r_{4}\right)$

$+1 x_{0 . . H}\left(\Delta r^{\prime}\right)^{2}+K^{\prime}{ }_{0 . . H} r^{\prime}{ }_{4}\left(\Delta r^{\prime}{ }_{4}\right)$

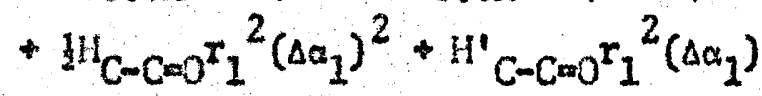

$+\mathrm{H}_{\mathrm{H}} \mathrm{C}-\mathrm{C} 0^{\mathrm{r}^{\prime}}{ }_{1}{ }^{2}\left(\Delta \alpha^{\prime}{ }_{1}\right)^{2}+\mathrm{H}^{\prime} \mathrm{C}-\mathrm{C}-\mathrm{O}^{\mathrm{r}^{\prime}}{ }_{1}{ }^{2}\left(\Delta \alpha^{\prime}{ }_{1}\right)$

$+1 H_{C-C-O} r_{2}^{2}\left(\Delta \alpha_{2}\right)^{2}+H_{C-C-O}{ }_{2}{ }^{2}\left(\Delta \alpha_{2}\right)$

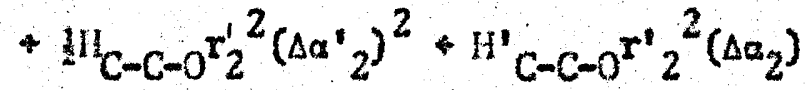

$+1 \mu_{0-C-O} r_{1} r_{2}\left(\Delta \alpha_{3}\right)^{2}+H_{0-C-0} r_{1} r_{2}\left(\Delta \alpha_{3}\right)$

$+{ }^{1} H_{0-C-O} \mathrm{r}^{\prime}{ }_{1}{ }^{r^{\prime}}{ }_{2}\left(\Delta \alpha^{\prime}{ }_{3}\right)^{2}+H_{0-C-O}{ }^{r^{\prime}}{ }_{1} \mathrm{r}_{2}{ }_{2}\left(\Delta \alpha^{\prime}{ }_{3}\right)$

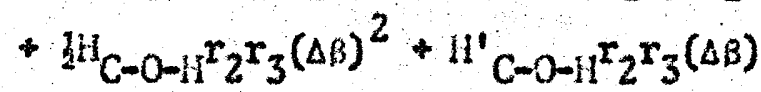

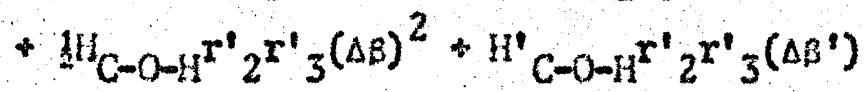

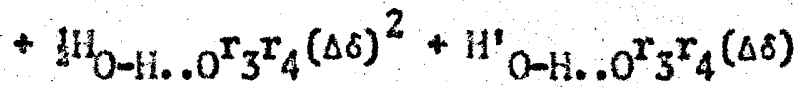

$+1 H_{0-H} .0^{r^{\prime}}{ }_{3}{ }^{\prime}{ }_{4}\left(\Delta \delta^{\prime}\right)^{2}+H_{0-H .} .0^{r^{\prime}}{ }_{3}{ }^{r^{\prime}}{ }_{4}\left(\Delta \delta^{\prime}\right)$

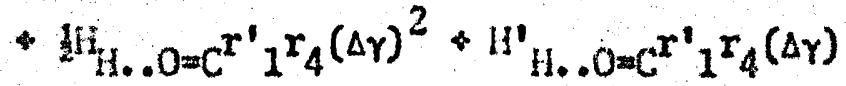

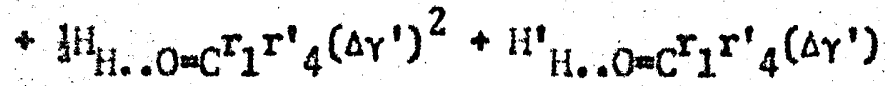

$+\frac{1}{2} F_{1,3}\left(\Delta q_{13}\right)^{2}+F_{1,3} q_{1,3}\left(\alpha_{31,3}\right)$

$+\frac{1}{2} F_{2,6}\left(\Delta q_{2,6}\right)^{2}+F_{2,6}^{\prime} q_{2,6}\left(\Delta q_{2,6}\right)$

$+1 F_{1,4}\left(\Delta q_{1,4}\right)^{2}+F_{1,4} q_{1,4}\left(\Delta q_{1,4}\right)$

$+1 \mathrm{~F}_{2,7}\left(\Delta q_{2,7}\right)^{2}+\mathrm{F}_{2,7} \mathrm{q}_{2,7}\left(\Delta q_{2,7}\right)$

- $1 F_{4,6}\left(\Delta q_{4,6}\right)^{2}+F_{4,6}^{\prime} q_{4,6}\left(\Delta q_{4,6}\right)$

$+\frac{1}{2} F_{3,7}\left(\Delta q_{3,7}\right)^{2}+F_{3,7}^{\prime} q_{3,7}\left(\Delta q_{3,7}\right)$

$+{ }_{2} F_{3,4}\left(\Delta q_{3,4}\right)^{2}+F_{3,4}^{\prime} q_{3,4}\left(\Delta q_{3,4}\right)$

$+1 \mathrm{~F}_{6,7}\left(\Delta q_{6,7}\right)^{2}+p^{\prime}{ }_{6,7} q_{6,7}\left(\Delta q_{6,7}\right)$

$+1 F_{1,5}\left(\Delta q_{1,5}\right)^{2}+F_{1,5}^{\prime} q_{1,5}\left(\Delta q_{1,5}\right)$

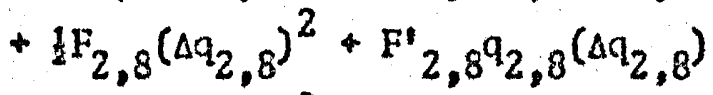

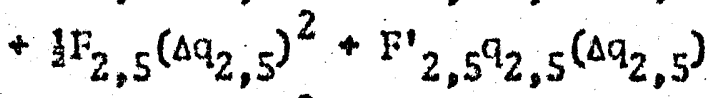

$+1 F_{1,8}\left(\Delta q_{1,8}\right)^{2}+F_{1,8} q_{1,8}\left(\Delta q_{1,8}\right)$ 
In which we assume that $F_{4,6}, F_{3,7}, F_{3,4}, F_{6,7}, F_{1,5}, F_{2,8}, F_{2,5}$ and $\mathrm{F}_{1,8}$ are small compared with any force constant above and wo neglect then to make the calculation possible.

Let

$$
\begin{aligned}
& F_{1,3}=F_{2,6}=F_{1} \\
& F_{1,4}=F_{2,7}=F_{2}
\end{aligned}
$$

From the assumption above there will be only 13 force constants in the potential energy expression of the inplane vibration of the oxalic acid molecule.

18.2 Evaluation of the F-gatrix

Elinination of $q$ was made using Table $I$ in section 6.3 and substituting all the $q$ values in the potential energy expression. The Urey-Bradley potential energy of the oxalic acid nolecule in tenns of intemal co-ordinatos becones

$$
\begin{aligned}
& V=\frac{1}{2}(\Delta R)^{2}\left(K_{C C}+S_{13}{ }^{2} F_{1}+t_{13}{ }^{2} F_{1}^{\prime}+s_{26}{ }^{2} F_{1}+t_{26}{ }^{2} F_{1}^{\prime}+s_{14}{ }^{2} F_{2}\right. \\
& \left.+t_{14}{ }^{2} \mathrm{~F}_{2}+\mathrm{S}_{27}{ }^{2} \mathrm{~F}_{2}+\mathrm{t}_{27}{ }^{2} \mathrm{~F} \cdot{ }_{2}\right) \\
& +\frac{1}{2}\left(\Delta r_{1}\right)^{2}\left(K_{C=0}+S_{31}{ }^{2} F_{1}+\varepsilon_{31}{ }^{2} F_{1}\right) \\
& +1\left(\Delta r_{1}^{\prime}\right)^{2}\left(K_{\mathrm{C}=0}+s_{62}{ }^{2} \mathrm{~F}_{1}+t_{62}{ }^{2} \mathrm{~F}_{1}\right) \\
& +\frac{1}{2}\left(\Delta r_{2}\right)^{2}\left(K_{C-O}+s_{41}{ }^{2} F_{2}+t_{41}{ }^{2} F_{2}\right) \\
& +\frac{1}{2}\left(\Delta r_{2}^{\prime}\right)^{2}\left(K_{C-O}+S_{72}{ }^{2} F_{2}+t_{72}{ }^{2} F_{2}^{\prime}\right) \\
& +\frac{1}{2}\left(\Delta r_{3}\right)^{2} K_{0-H} \\
& +1\left(\Delta r_{3}^{\prime}\right)^{2} \mathrm{~K}_{\mathrm{O}-\mathrm{i}} \\
& +1\left(\Delta r_{4}\right)^{2} K_{0 . H} \\
& +\frac{1}{2}\left(\Delta r_{4}^{\prime}\right)^{2} K_{0 . .11} \\
& \text { - } \frac{1}{2}\left(r_{1} \Delta a_{1}\right)^{2}\left({ }_{C-C=0}+\left(R / r_{1} t_{13} t_{31} F_{1}-\left(R / r_{1} S_{13} S_{31} F_{1}\right)\right.\right. \\
& \text { - } \frac{1}{2}\left(r^{\prime}{ }_{1} A C^{\prime}{ }_{1}\right)^{2}\left(l_{C-C=0}+\left(R / r^{\prime}{ }_{1} t_{26} C_{62} F_{1}-\left(R / r^{\prime}{ }_{1} S_{26} S_{62}{ }^{\prime \prime}{ }_{1}\right)\right.\right.
\end{aligned}
$$




$$
\begin{aligned}
& +\frac{1}{2}\left(\mathrm{r}_{2} \Delta \mathrm{a}_{2}\right)^{2}\left(\mathrm{H}_{\mathrm{C}-\mathrm{C}-\mathrm{O}}+\left(\mathrm{R} / \mathrm{r}_{2} \mathrm{t}_{14} \mathrm{t}_{41} \mathrm{~F}_{2}-\left(\mathrm{R} / \mathrm{r}_{2} \mathrm{~S}_{14} \mathrm{~S}_{41} \mathrm{~F}_{2}{ }_{2}\right)\right.\right. \\
& +\frac{1}{2}\left(r^{\prime}{ }_{2}{ }^{\Delta \alpha^{\prime}}{ }_{2}\right)^{2}\left(\mathrm{H}_{\mathrm{C}-\mathrm{C}-\mathrm{O}}+\left(\mathrm{R} / \mathrm{r}^{\prime}{ }_{2}\right)_{27} \mathrm{t}_{72} \mathrm{~F}_{2}-\left(\mathrm{R} / \mathrm{r}_{2}{ }_{2} \mathrm{~S}_{27} \mathrm{~S}_{72} \mathrm{~F}^{\prime}{ }_{2}\right)\right. \\
& +\frac{1}{2} r_{1} r_{2}\left(\Delta \alpha_{3}\right)^{2} H_{0-C}=0 \\
& +\frac{1}{2} r_{1}^{\prime}{ }^{\prime}{ }_{2}\left(\Delta \alpha^{\prime}{ }_{3}\right)^{2} \mathrm{H}_{0-\mathrm{C}=0} \\
& +\frac{1}{2} \mathrm{r}_{2} \mathrm{r}_{3}(\Delta \beta)^{2} \mathrm{H}_{\mathrm{C}-\mathrm{O}-\mathrm{H}} \\
& +\frac{1}{2} r_{2}{ }_{2}{ }^{\prime}{ }_{3}\left(\Delta \beta^{\prime}\right)^{2}{ }^{H} \mathrm{C}-\mathrm{O}-\mathrm{H} \\
& +\frac{1}{2} r_{3} r_{4}(\Delta \delta)^{2} \mathrm{H}_{0-\mathrm{H}_{0} .0} \\
& +\frac{1}{2} \mathrm{r}^{\prime}{ }^{\mathrm{r}^{\prime}}{ }_{4}\left(\Delta \delta^{\prime}\right)^{2} \mathrm{H}_{\mathrm{O}-\mathrm{H} . . \mathrm{O}} \\
& +\frac{1}{2} r^{\prime}{ }_{1} r_{4}(\Delta \gamma)^{2} H_{H} . .0=C \\
& +\frac{1}{2} r_{1} r_{4}^{\prime}\left(\Delta \gamma^{\prime}\right)^{2} \mathrm{H}_{\mathrm{H} . . \mathrm{O}=\mathrm{C}} \\
& +(\Delta R)\left(\Delta r_{1}\right)\left(S_{13} S_{31} F_{1}-t_{13} t_{31} F_{1}^{\prime}\right) \\
& +(\Delta R)\left(\Delta r_{1}^{\prime}\right)\left(S_{26} S_{62} F_{1}-t_{26} t_{62} F_{1}^{\prime}\right) \\
& +(\Delta R)\left(\Delta r_{2}\right)\left(S_{14} S_{41} F_{2}-t_{14} t_{41} F_{2}^{\prime}\right) \\
& +(\Delta R)\left(\Delta r_{2}^{\prime}\right)\left(S_{27} S_{72} F_{2}-t_{27} t_{72} F_{2}^{\prime}\right) \\
& \left.+(\Delta R)\left(r_{1} \Delta \alpha_{1}\right)\left(\sqrt{R / r_{1}}\right) S_{13} \sqrt{t_{13} t_{31}} F_{1}+t_{13} S_{31} F_{1}\right)
\end{aligned}
$$

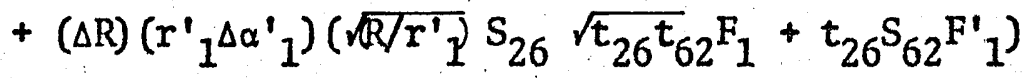

$$
\begin{aligned}
& +(\Delta R)\left(r_{2} \Delta \alpha_{2}\right)\left(\sqrt{R / r_{2}} S_{14} \sqrt{t_{14} t_{41}} F_{2}+t_{14} S_{41} F_{2}\right) \\
& +(\Delta R)\left(r_{2}^{\prime}{ }_{2} \alpha_{2}^{\prime}\right)\left(R / r^{\prime} S_{27} \sqrt{t_{27} t_{72}} F_{2}+t_{27} S_{72} F_{2}^{\prime}\right) \\
& +\left(\Delta r_{1}\right)\left(r_{1} \Delta \alpha_{1}\right)\left(\sqrt{\left.R / r_{1}\right)} S_{31} \sqrt{t_{13} t_{31}} F_{1}+\left(R / r_{1}\right) t_{31} S_{13} F^{\prime}{ }_{1}\right) \\
& \left.+\left(\Delta r_{1}^{\prime}\right)\left(r^{\prime}{ }_{1} \Delta \alpha^{\prime}{ }_{1}\right)\left(\sqrt{\left(R / r^{\prime}{ }_{1}\right.}\right) S_{62} \sqrt{t_{26} t_{62}} F_{1}+\left(R / r_{1}^{\prime}\right) t_{62} S_{26} F^{\prime}{ }_{1}\right) \\
& \left.+\left(\Delta \mathrm{r}_{2}\right)\left(\mathrm{r}_{2} \Delta \alpha_{2}\right)\left(\sqrt{R / \mathrm{r}_{2}}\right) S_{41} \sqrt{\mathrm{t}_{14} \mathrm{t}_{41}} \mathrm{~F}_{2}+\left(\mathrm{R} / \mathrm{r}_{2}\right) \mathrm{t}_{41} \mathrm{~S}_{14} \mathrm{~F}_{2}\right) \\
& +\left(\Delta r_{2}^{\prime}\right)\left(r^{\prime}{ }_{2}^{\Delta \alpha^{\prime}}{ }_{2}\right)\left(\sqrt{\left.R / r^{\prime}{ }_{2}\right)} S_{72} \sqrt{t_{27} t_{72}} F_{2}+\left(R / r^{\prime}{ }_{2} t_{72} S_{27} F_{2}\right)\right.
\end{aligned}
$$

in which

$$
\begin{array}{ll}
s_{13}=s_{26}=0.909048 & t_{13}=t_{26}=0.416692 \\
s_{31}=s_{62}=0.845502 & t_{31}=t_{62}=0.533972
\end{array}
$$




$$
\begin{array}{ll}
s_{14}=s_{27}=0.854918 & t_{14}=t_{27}=0.518762 \\
s_{41}=s_{72}=0.80020 & t_{41}=t_{72}=0.599734
\end{array}
$$

\subsection{F-matrix in intemal co-ondinates of the gaseous oxalic acid}

\section{2nd mode1}

$$
\begin{aligned}
& F(1,1)=K_{1}+1.61801 F_{1}+1.407947 F_{2} \\
& F(1,2)=0.790852 F_{1} \quad F(1,3)=0.790852 F_{1} \\
& F(1,4)=0.715217 \mathrm{~F}_{2} \quad F(1,5)=0.715217 \mathrm{~F}_{2} \\
& F(1,10)=0.450175 F_{1} \quad F(1,11)=0.471212 F_{2} \\
& F(1,13)=0.450175 F_{1} \quad F(1,14)=0.471212 F_{2} \\
& F(1,6)=F(1,7)-F(1,8)=F(1,9)=F(1,12)=F(1,15)=F(1,16) \\
& =F(1,17)=F(1,18)=F(1,19)=F(1,20)=F(1,21)=0 \\
& F(2,1)=0.790852 F_{1} \quad F(2,2)=K_{2}+0.686361 F_{1} \\
& F(2,10)=0.389272 F_{1} \quad F(2,3)=F(2,4)=F(2,5)=F(2,6) \\
& \text { - } F(2,7)=F(2 n 8)=F(2,9)=F(2,11)-F(2,12)-F(2,13)=F(2,14) \\
& =F(2,15)=F(2,16)=F(2,17)=F(2,18)=F(2,19)=F(2,20)=F(2,21) \\
& =0 \\
& F(3,1)=0.790852 F_{1} \quad F(3,3)-K_{2}+0.686361 F_{1} \\
& F(3,13)=0.389272 F_{1} \quad F(3,2)=F(3,4)=F(3,5)=F(3,6) \\
& \text { - } F(3,7)=F(3,8)-F(3,9)=F(3,10)=F(3,11)=F(3,12)=F(3,14) \\
& \text { - } F(3,15)-F(3,16)=F(3,17)=F(3,18)-F(3,19)=F(3,20)=F(3,21) \\
& =0
\end{aligned}
$$




$$
\begin{aligned}
& F(4,1)=0.715217 F_{2} \quad F(4,4)=K_{3}+0.604352 F_{2} \\
& F(4,11)=0.420632 F_{2} \quad F(4,2)=F(4,3)=F(4,5)=F(4,0) \\
= & F(4,7)=F(4,8)=F(4,9)-F(4,10)=F(4,12)=F(4,13)=F(4,14) \\
= & F(4,15)=F(4,16)=F(4,17)=F(4,18)=F(4,19)=F(4,20) \\
= & F(4,21)=0 \\
& F(5,1)=0.715217 F_{2} \quad F(5,5)=K_{3}+0.604352 F_{2} \\
& F(5,14)=0.420632 F_{2} \quad F(5,2)=F(5,3)=F(5,4)=F(5,0) \\
= & F(5,7)=F(5,8)=F(5,9)=F(5,10)=F(5,11)=F(5,12)=F(5,13) \\
= & F(5,15)=F(5,16)=F(5,17)=F(5,18)=F(5,19)=F(5,20)=F(5,21) \\
= & 0
\end{aligned}
$$

$$
\begin{aligned}
& F(6,6)=K_{4} \\
= & F(6,5)-F(6,7)=F(6,8)=F(6,9)=F(6,10)=F(6,11)=F(6,12) \\
= & F(6,13)=F(6,14)=F(6,15)=F(6,16)-F(6,17)=F(6,18) \\
- & F(6,19)=F(6,20)=F(6,21)=0
\end{aligned}
$$

$$
\begin{aligned}
& F(7,7)=K_{4} \quad F(7,1)=F(7,2)=F(7,3)=F(7,4) \\
= & F(7,5)=F(7,6)=F(7,8)-F(7,9)=F(7,10)=F(7,11)=F(7,12) \\
= & F(7,13)=F(7,14)-F(7,15)=F(7,16)=F(7,17)=F(7,18) \\
= & F(7,19)=F(7,20)=F(7,21)=0
\end{aligned}
$$

$$
\begin{aligned}
& F(8,8)=K_{5} \quad F(8,1)=F(8,2)=F(8,3)=F(8,4) \\
= & F(8,5)=F(8,6)=F(8,7)=F(8,9)-F(8,10)=F(8,11)=F(8,12) \\
= & F(8,13)=F(8,14)=F(8,15)=F(8,16)=F(8,17)=F(8,18)=F(8,19) \\
= & F(8,20)=F(8,21)=0
\end{aligned}
$$




$$
\begin{aligned}
& F(9,9)=K_{5} \quad F(9,1)=F(9,2)=F(9,3)=F(9,4) \\
= & F(9,5)=F(9,5)=F(9,6)=F(9,7)=F(9,8)=F(9,10)=F(9,11) \\
= & F(9,12)=F(9,13)=F(9,14)=F(9,15)=F(9,16)=F(9,17) \\
= & F(9,18)=F(9,19)=F(9,20)=F(9,21)=0
\end{aligned}
$$

$$
\begin{aligned}
& F\left(10,11=0.450175 F_{1} \quad F(10,2)=0.389272 F_{1}\right. \\
& F(10,10)=H_{1}+0.385620 F_{1} F(10,3)=F(10,4)-F(10,5) \\
&= F(10,0)=F(10,7)=F(10,8)=F(10,9)=F(10,11)=F(10,12) \\
&=F(10,13)=F(10,14)-F(10,15)=F(10,16)=F(10,17)=F(10,18) \\
&=F(10,19)=F(10,20)=F(10,21)=0
\end{aligned}
$$

$$
\begin{aligned}
& F(11,1)=0.471212 F_{2} \quad F(11,4)=0.420532 F_{2} \\
& F(11,11)=l_{2}+0.438769 F_{2} F(11,2)=F(11,3)=F(11,5) \\
= & F(11,6)=F(11,7)=F(11,8)=F(11,9)=F(11,10)=F(11,12) \\
= & F(11,13)=F(11,14)-F(11,15)=F(11,16)=F(11,17)=F(11,18) \\
= & F(11,19)=F(11,20)=F(11,21)=0
\end{aligned}
$$

$$
\begin{aligned}
& F(12,12)=H_{3} \quad F(12,1)=F(12,2)=F(12,3)=F(12,4) \\
& =F(12,5)=F(12,6)=F(12,7)=F(12,8)=F(12,9)=F(12,10)-F(12,11) \\
& =F(12,13)=F(12,14)=F(12,15)=F(12,16)=F(12,17)-F(12,18) \\
& =F(12,19)=F(12,20)=F(12,21)=0 \\
& F(13,1)=0.450175 F_{1} \quad F(13,3)=0.389272 F_{1} \\
& F(13,13)=H_{1}+0.383620 F_{1} \quad F(13,2)=F(13,4)=F(13,5)=F(13,0) \\
& =F(13,7)=F(13,8)=F(13,9)=F(13,10)=F(13,11)=F(13,12) \\
& =F(13,14)=F(13,15)=F(13,16)=F(13,17)=F(13,18)=F(13,19) \\
& =F(13,20)=F(13,21)=0
\end{aligned}
$$




$$
\begin{aligned}
& F(14,1)=0.471212 P_{2} \quad F(14,5)=0.420532 P_{2} \\
& F(14,14)=H_{2}+0.438769 F_{2} F(14,2)=F(14,3)=F(14,4) \\
& =F(14,6)=F(16,7)=F(14,8)=F(14,9)=F(14,10)=F(14,11) \\
& =F(14,12)=F(14,13)=F(14,15)=F(14,16)=F(14,17)=F(14,18) \\
& =F(14,19)=F(14,20)=F(14,21)=0 \\
& F(15,15)=H_{3} \quad F(15,1)=F(15,2)=F(15,3) \\
& =F(15,4)=F(15,5)=F(15,6)=F(15,7)=F(15,8)=F(15,9) \\
& =F(15,10)=F(15,11)=F(15,12)=F(15,13)=F(15,14)=F(15,16) \\
& =F(15,17)=F(15,18)-F(15,19)-F(15,20)=F(15,21)=0 \\
& F(16,16)=H_{4} \quad F(16,1)=F(16,2)=F(16,3) \\
& =F(16,4)=F(16,5)=F(16,6)=F(16,7)=F(16,8)=F(16,9) \\
& \text { - } F(16,10)-F(16,11)=F(16,12)=F(16,13)-F(16,14)-F(16,15) \\
& =F(16,17)-F(16,18)-F(10,19)=F(16,20)=F(16,21)=0 \\
& F(17,17)=H_{5} \quad F(17,1)=F(17,2)=F(17,3) \\
& =F(17,4)=F(17,5)=F(17,6)=F(1.7,7)=F(17,8)=F(17,9) \\
& =F(17,10)=F(17,11)=F(17,12)=F(17,13)=F(17,14)=F(17,15) \\
& =F(17,16)=F(17,18)=F(17,19)=F(17,20)=F(17,21)=0 \\
& F(18,18)=H_{6} \quad F(18,1)=F(18,2)=F(18,3)=F(18,4) \\
& =F(18,5)=F(18,6)=F(18,7)=F(18,8)=F(18,9)=F(18,10)=F(18,11) \\
& =F(18,12)=F(18,13)=F(18,18)=F(18,15)=F(18,16)=F(18,17) \\
& \text { - } F(18,19)=F(18,20)=F(18,21)=0
\end{aligned}
$$




$$
\begin{aligned}
& F(19,19)=H_{4} \quad F(19,1)=F(19,2)=F(19,3) \\
& =F(19,4)=F(19,5)=F(19,6)=F(19,7)=F(19,8)=F(19,9) \\
& =F(19,10)=F(19,11)=F(19,12)=F(19,13)=F(19,14)=F(19,15) \\
& =F(19,16)-F(19,17)-F(19,18)-F(19,20)=F(19,21)=0
\end{aligned}
$$

$$
\begin{aligned}
& F(20,20)=H_{5} \quad F(20,1)=F(20,2)=F(20,3) \\
& =F(20,4)=F(20,5)-F(20,6)-F(20,7)=F(20,8)=F(20,9) \\
& =F(20,10)=F(20,11)=F(20,12)-F(20,13)-F(20,14)=F(20,15) \\
& =F(20,16)=F(20,17)=F(20,18)=F(20,19)=F(20,21)=0 \\
& F(21,21)=H_{6} \quad F(21,1)=F(21,2)=F(21,3) \\
& =F(21,4)=F(21,5)=F(21,6)=F(21,7)=F(21,8)=F(21,9) \\
& =F(21,10)=F(21,11)=F(21,12)=F(21,13)=F(21,14)=F(21,15) \\
& =F(21,16)=F(21,17)=F(21,18)=F(21,19)=F(21,20)=0
\end{aligned}
$$

$$
\begin{aligned}
& \text { in which } K_{1}=K_{C C} \quad K_{2}=K_{C=O} \\
& \mathrm{~K}_{3}=\mathrm{K}_{\mathrm{C}-\mathrm{O}} \quad \mathrm{K}_{4}=\mathrm{K}_{\mathrm{O}-\mathrm{H}} \\
& K_{5}=K_{0 . . H} \quad H_{1}=H_{C-C=O} \\
& \mathrm{H}_{2}=\mathrm{H}-\mathrm{C}-\mathrm{O} \quad \mathrm{H}_{3}=\mathrm{H}_{\mathrm{O}-\mathrm{COO}} \\
& \mathrm{H}_{4}=\mathrm{H}_{\mathrm{C}-\mathrm{O}-\mathrm{IH}} \quad \mathrm{H}_{5}=\mathrm{H}_{0-\mathrm{H} . .0} \\
& \mathrm{H}_{6}=\mathrm{H}_{\mathrm{H} . . . \mathrm{O}=\mathrm{C}} \quad \mathrm{F}_{1}=\mathrm{F}_{\mathrm{C}_{1} \mathrm{O}_{3}}-\mathrm{F}_{\mathrm{C}_{2} \mathrm{O}_{6}} \\
& \mathrm{~F}_{2}-\mathrm{F}_{\mathrm{C}_{1} \mathrm{O}_{4}}=\mathrm{F}_{\mathrm{C}_{2} \mathrm{O}_{7}}
\end{aligned}
$$


18.4 F-matrix in symetry co-ordinates of gascous oxalic acid

(2nd mode1)

$$
\begin{aligned}
& \mathrm{F}(1,1)=\mathrm{K}_{1}+1.61801 \mathrm{~F}_{1}+1.407947 \mathrm{~F}_{2} \\
& \mathrm{~F}(1,2)=1.118423 \mathrm{~F}_{1} \quad \mathrm{~F}(1,3)=1.01147 \mathrm{~F}_{2} \\
& \mathrm{M}(1,6)=-0.259909 \mathrm{~F}_{1}-0.272054 \mathrm{~F}_{2} \\
& \operatorname{Fr}(1,4)=\operatorname{FM}(1,5)=\operatorname{MM}(1,7)=\operatorname{Fr}(1,8)-F(1,9)=\operatorname{Fr}(1,10) \\
& =\operatorname{F}(1,11)=\operatorname{IM}(1,12)=\operatorname{MM}(1,13)=0 \\
& F(2,1)=1.118434 F_{1} \quad F(2,2)=K_{2} * 0.686361 F_{1} \\
& F(2,6)=0.15892 F_{1} \quad \operatorname{Fr}(2,3)=\operatorname{F(2,4)}=\operatorname{M}(2,5)
\end{aligned}
$$

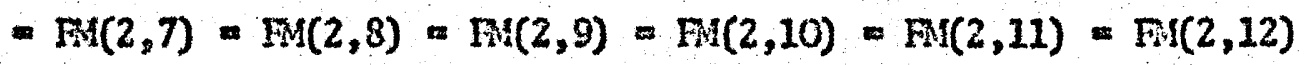

$$
\begin{aligned}
& \text { - } M(2,13)=0 \\
& F(3,1)=1.01147 F_{2} \quad M(3,3)=K_{3}+0.604352 F_{2} \\
& \operatorname{FM}(3,6)=-0.171722 F_{2} \quad \operatorname{FM}(3,2)=\operatorname{FM}(3,4)=\operatorname{FM}(3,5) \\
& -\operatorname{FM}(3,7)=\operatorname{FM}(3,8)-\operatorname{FM}(3,9)=\operatorname{FM}(3,10)-\operatorname{FM}(3,11)-\operatorname{FM}(3,12) \\
& =F(3,13)=0
\end{aligned}
$$

$$
\begin{aligned}
& \operatorname{FM}(4,4)=x_{4} \quad \operatorname{FM}(4,1)=\operatorname{WM}(4,2)=\operatorname{FM}(4,3) \\
& \text { - } \left.\operatorname{Fr}(4,5)=\mathrm{P}_{(4,6)-\operatorname{WM}(4,7)-\operatorname{Fr}} \quad 1,10\right) \\
& =F(4,11)=F M(4,12)=F(4,13)=0 \\
& \operatorname{Mi}(5,5)=K_{5} \quad \operatorname{PF}(5,1)=\operatorname{FN}(5,2)=\operatorname{mM}(5,3) \\
& =\operatorname{MM}(5,4)=\operatorname{Fi}(5,6)=\operatorname{FM}(5,7)=\operatorname{MM}(5,8)=\operatorname{MM}(5,9)=\operatorname{Mr}(5,10) \\
& \text { - } M(5,11)-M(5,12)=M(5,13)=0
\end{aligned}
$$




$$
\begin{aligned}
& \operatorname{Fin}(12,8)=-0.15392 F_{1} \quad F(12,9)=-0.171722 F_{2} \\
& F(12,12)=\mathrm{H}_{1} / 6+\mathrm{H}_{2} / 6+\frac{2}{3} \mathrm{H}_{3}+0.063937 \mathrm{~F}_{1}+0.073128 \mathrm{~F}_{2} \\
& W(12,1)=\operatorname{Fo}(12,2)=M(12,3)=\operatorname{Mm}(12,4)=\operatorname{M}(12,5)=M(12,0) \\
& \text { - } \operatorname{Fin}(12,7)=\operatorname{FH}(12,10)=\operatorname{Fat}(12,11)=\operatorname{Br}(12,13)=0 \\
& \operatorname{rm}(13,13)=I_{4} / 6+\frac{2}{3} \mathrm{H}_{5}+\frac{1}{6} \mathrm{H}_{6}
\end{aligned}
$$

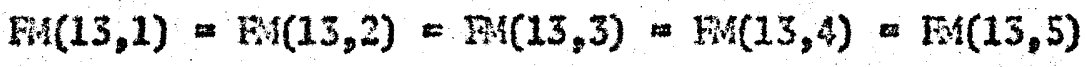

$$
\begin{aligned}
& \text { - } \operatorname{F}(13,6)=\operatorname{Mr}(13,7)=N(13,8)=\operatorname{PA}(13,9)=\operatorname{PM}(13,10) \\
& =\operatorname{Fr}(13,12)=\operatorname{Fin}(13,12)=0
\end{aligned}
$$


CAATTER 19

THE TNFRARED RXPERTMGNT

\subsection{The infrared instrument}

The infrared spectrum was recorded with a perkin Elmer 457 infrared spectroneter.

As shown on Fig. 23, the radiation eraitted by the source is split into the sample and the reference beans by the plane mirror $M_{1}$ and toroidal mirrors $M_{2}$ and $M_{3}$. The toroidal nirror $M_{2}$ focuses the sample been on to the 100 conb, and $M_{3}$ focuses the reference beam onto the servo-controlled optical wedge. To ensure that the optical system is uniforaly filled, the source inage produced by the toroids is double size, i.e. 6 m wide. The $100 \%$ conbl is used to adjust the intensity of the sample beam so that pen indicates $100 \%$ transnittance when no sample is present. When the 100 corb is noved into or out of the sample berus, the servo-notor moves the optical wedge in the reference bean to equalize the bear intensities, its movenent being followed by the pen. The sanple and the reference boans are conbined by the rotating sector mirror to follow a conton opticnl path. This combined bean is then focused on the nonocluronator entrance slit by toroidel mirror $7^{\circ}$. The bean is dispersed by the grating into its spectral components, Rotation of the grating results in the dispersed spectrun being scanned across the monochronator slit. The machanical width of the nonochromator slit deternines the width of tho wavenubber band energing from the nonochromator. Thus, docreasing the slit width decreases both band width and the 


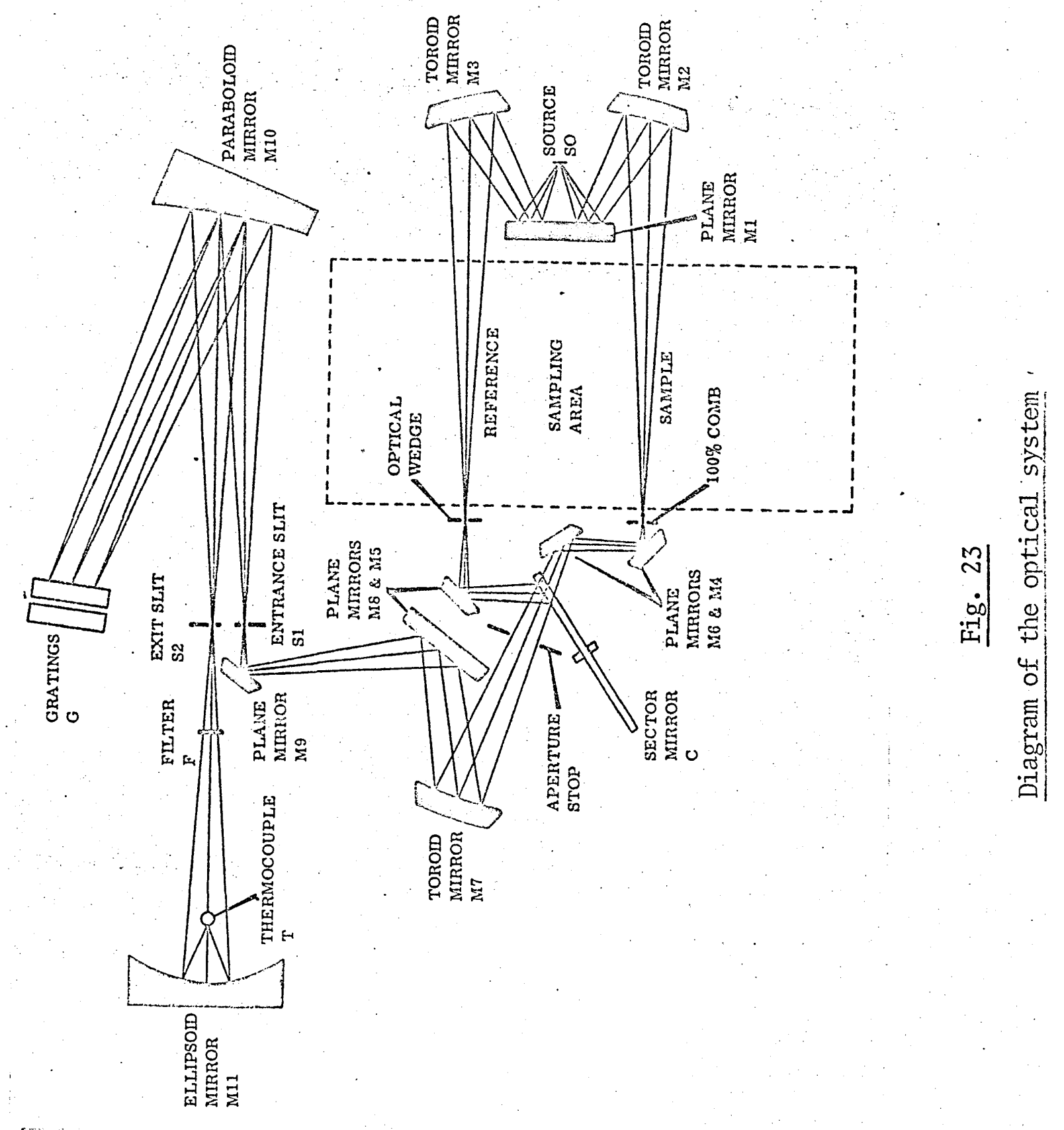


intensity of the energing radiation (i.o. decreases signal to noise ratio). The slit vidth is aljusted by the slit control on the front panel. After leaving the monociromator the radiation passes trmough one of a set of optical filters, the correct filter being autonatically selected for the spectral region beling scanned. This filter absorbs unanted radiation diffracted from the grating at the samo angle as the component of the desired wave numbor. Finally the transnitted radiation is focused onto a therrocouple detector. The altemating signal on the detector is amplified and fed to a servomotor which moves the reference been altenuator to equalize the intensity of the sarsple and reference beans. The altemating signal is thereby reduced to zero, producing a state of equilibrium.

At the characteristic absorption frequencies of the sample, the intensity of the sample beam changes and an altemating signal cailed the BRROR signal is then generated by the detector, the amplitude of this signal being proportional to the orror between the true transmittance and the indicated transmittance. The error signal is amplified by the $11 \mathrm{~Hz}$ amplifier, rectified by the synchronous rectifier, smothed and rodulated to give a $50 \mathrm{~Hz}$ signal the phase of which is dependent on which bear is the more intense. After further amplification by the min anplifier chiss signal is used to drive tho servo-motor in a direction depending on the phase of the $50 \mathrm{~Hz}$ sigalal so that the reference bean attenuator is maved to equalize the intensity of the sample and reference beans, thus roducing the orror signal to zero. Since the recorder pen is mechanically linked to the attenuetor, the pen will be displaced by an emount proportional to the change in sample transmittance. 


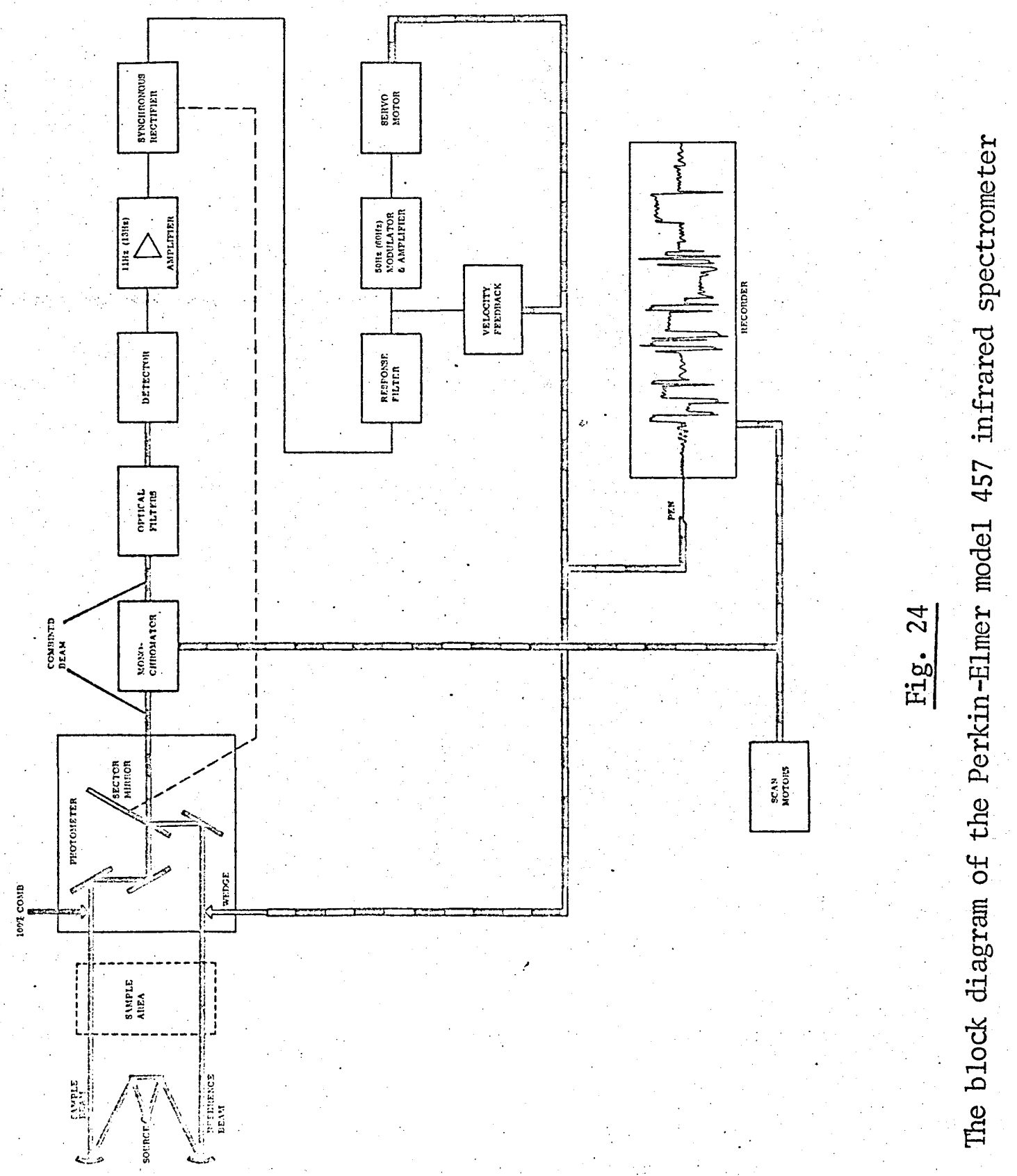


19.2 Devoloping the heated coll and the aditional optical syston

The hested cell is mede of stainless steel of cylindrical shape $30 \mathrm{~cm}$ in longth, $5 \mathrm{~cm}$ in dianeter, and the wall $0.9 \mathrm{~cm}$ thick, with two viton ' $O$ ' rings fitted on both onds. Two kBr windows are held over ' $O$ ' rings by means of the scren-ou caps. The electrical heating element of the cell body is provided with e power supily which can be varied by moans of a variac. The cell body is covered by the themal shield. There are also a high vacum valve, a silficone rubber septun and a snall thermocouple junction hole provided an the cell body.

The X-section of the infrared cell is show in Fig. 25 . In the beginning, two pieces of cylindrical copper pipe with heating wires were fitted to both whdows to prevent excessive cooling of the windors and condensation of the sample (see Fig. 27).

An additional optical systen is required because the length of the sample compartment of the Perkin-11mar 457 infrared spectrometer is shorter than tho length of the heated cell. As shown in Fig. 27, the plane nirror $A$ roflects the sample bean tirough the cell and the concave nirror $B$ at the beck of the cell focuses the radiation bachwards hitting the plane mirror $\mathrm{C}$ which will reflect the radiation through the 1008 conb.

An advantage of this optleal system is that the sample path length is twice the length of the coll. However, sevarnl disadvantages ariso as follows:- 


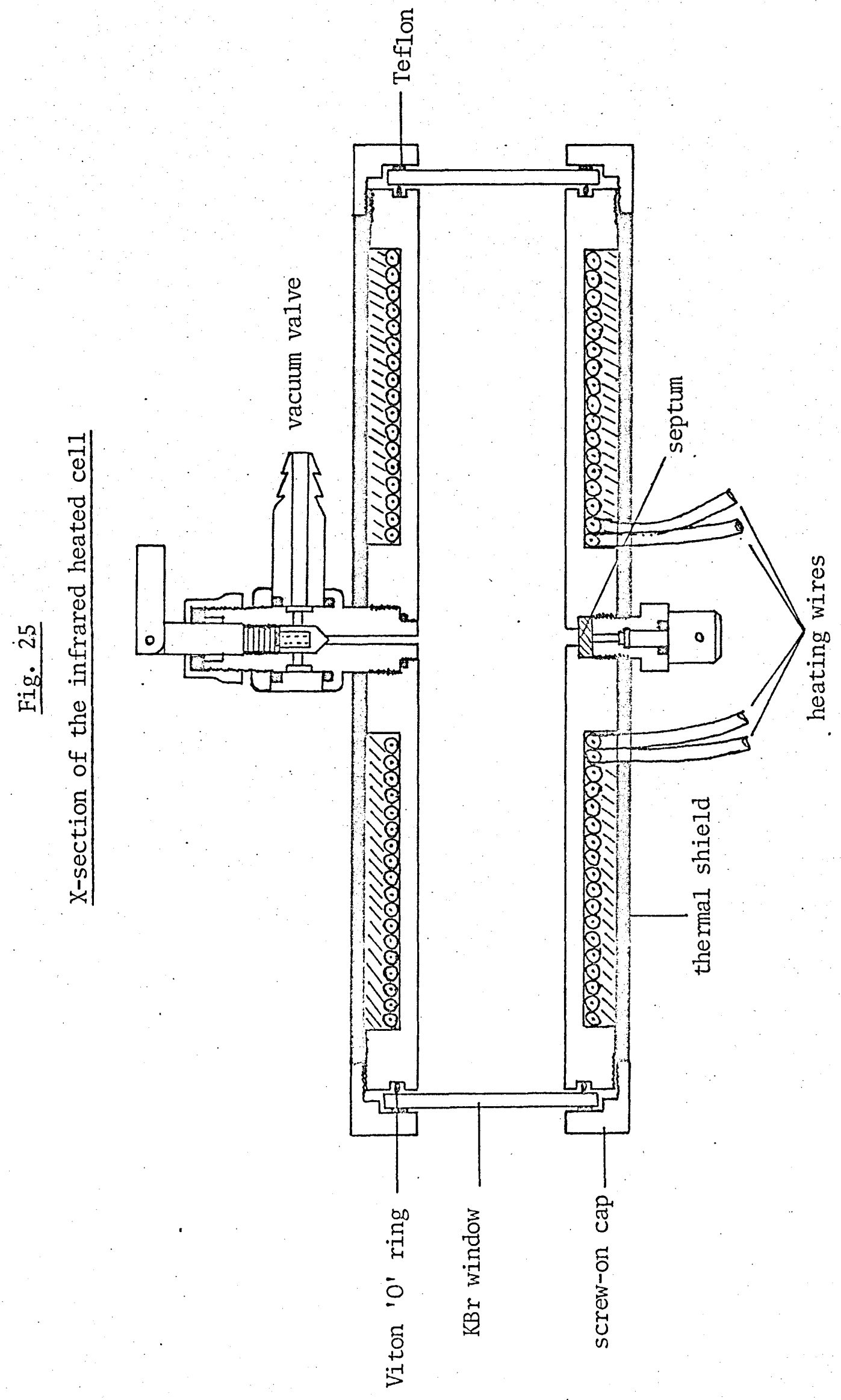


(1) The air path lengths of the sample beam and the reference beam are different. Thus, the spectrum of $\mathrm{CO}_{2}$ and $\mathrm{H}_{2} \mathrm{O}$ in air are added to the sample spectrum.

(2) There will be some energy loss at the surfaces of the A, $\mathrm{B}$, and $\mathrm{C}$ mirrors, since their reflectivity is not $100 \%$.

(3) There will be considerable reflection losses at the windows as each window is traversed twice.

(4) There will be some energy loss caused by the size of the ce11. The following calculation shows the approximate percentage of energy loss at the position of the second window, since the effective area of the second window is smaller than the area of the beam.

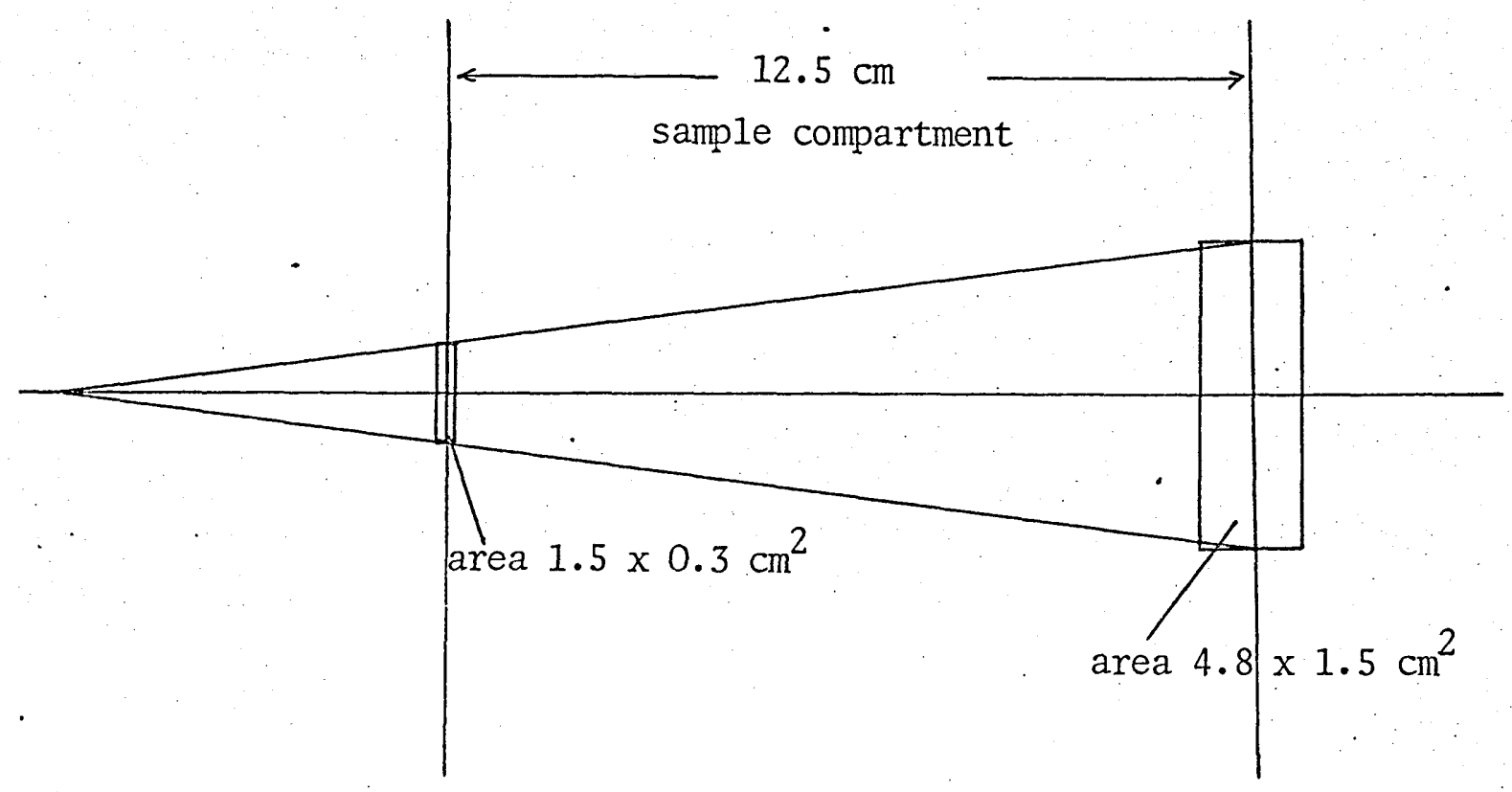

Fig. 26

The effective area of the beam at the sample compartment 
From Fig. 27 object distance $=\quad u \mathrm{~cm}$

$\begin{array}{ll}\text { inige distance } v= & u+9.3+7.7 \mathrm{~cm} \\ \text { focal length of the } & \\ \text { concave nirror }= & 20 \mathrm{~cm} \\ \therefore \quad u= & 33.2 \mathrm{~cm}\end{array}$

Let the $2 \mathrm{nd} \mathrm{KBr}$ window be at $4.2 \mathrm{~cm}$ from the concave mirror

$\therefore$ the wideh of the inage at the 2 nd window $m 2.8 \mathrm{~cm}$ the height of the inage at the $2 \mathrm{nd}$ window $=9.0 \mathrm{~cm}$ the bean area of the image at the $2 \mathrm{nd}$ window $=25.2 \mathrm{~cm}^{2}$ From Fig. 2Sa the area of the bean passing through the second winciow $=$ $13.26 \mathrm{~cm}^{2}$ $\therefore$ the energy loss = 47.25

Similarly, if the second window is at $7.2 \mathrm{~cm}$ from the concave nirror, the area of the bean at the second window is $20 \mathrm{~cm}^{2}$ and the area of the bean passing through the second window (fron Fig. 28b) is $11.92 \mathrm{~cm}^{2}$. Thus the energy loss is 40.48 . The calculation above shows that the closer the heatod cell is to the plane nirrors $A$ and $C$, the snaller the energy loss. In order to increase the transmittance of the bean and also reduce the condensation problem, the second $\mathrm{KBr}$ window was replaced by a concave nitror. The radius of curvature of $33.7 \mathrm{~cm}$ was chosen for maximum transmitcance and the mirror was specially made. The concave nirror is hold over a viton rubber ' $O$ ' ring by neans of a screw-on cap to which an electric heater was fixed (see $\mathrm{Fig} \cdot 29$ ). 
The final heated cell is shom in Fig. 29. A not vacuun outlet was nade with an electric heater attached to it in order to prevent condensation of the oxalic acle taking place in the outlet. 

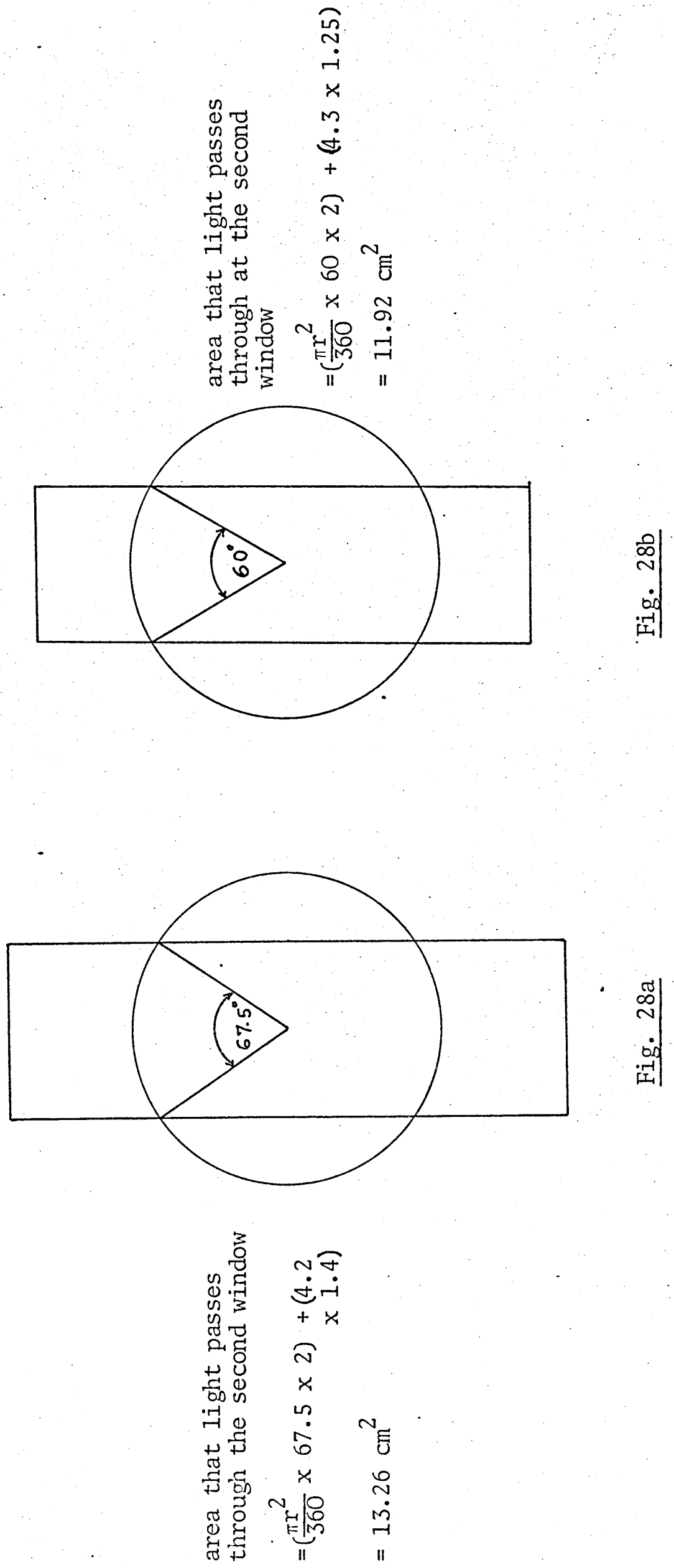


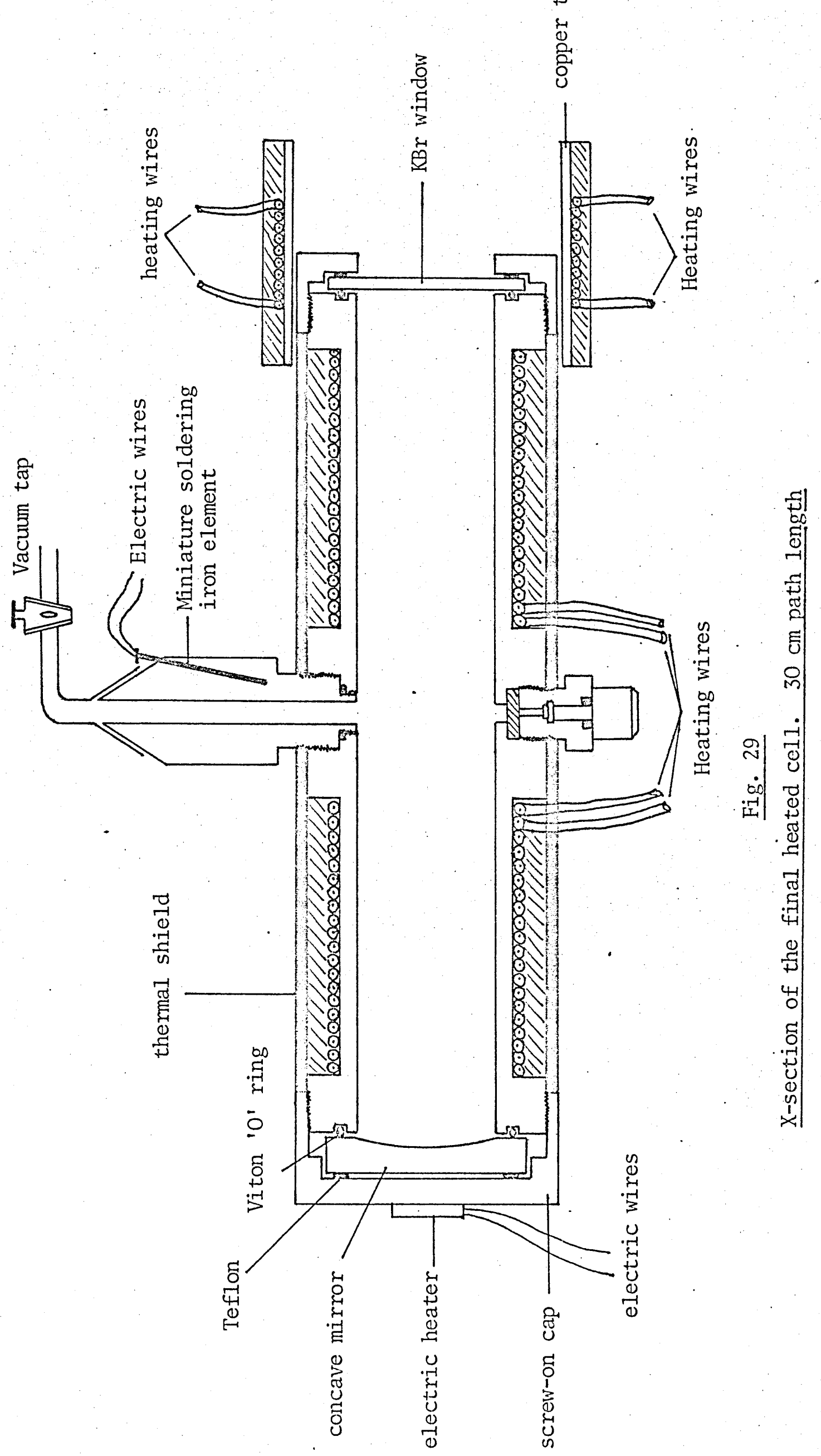




\subsection{Infrarod experiment}

At the beginning of the work the experiment was performed by placing some anhydrous oxalic acid in the heated cell and evacuating to eliminate the air spectrum. After pumping for a while the vacum valve was sealed and the coll was slowly heated up. The spectrum recorded shows that

(a) the oxalic acid started to subline at $65^{\circ} \mathrm{C}$ and slowly condensed on both $\mathrm{KBr}$ windows. The condensation process is faster at higher temporatures. This happened because the windows temperature is lower than the coll body temperature;

(b) at temperatures above $65^{\circ} \mathrm{C}$ the amount of the decomposition products increased with increase in temperature. These waste products produced quite a high background spectrun. Thus, handiing the sample in ehis way the spectron can only be recorded up to $65^{\circ} \mathrm{C}$. At terperatures higher than this the condensation of the oxalic acid on both $\mathrm{KBr}$ windows and the decomposition products make the gaseous oxalic acid bands undetectable. It is obvious that both problems need to be eliminated. In order to elininate the condensation problem, two cylindrical copper pipes fitted with electrical heating wires were attached on both ends of the heated cell to keop the $\mathrm{KBr}$ window terperature higher than the cell body. It is reasonable that the $\mathrm{KBr}$ windows should be warmed before henting un the cell body. 
The experinene was porformed again by evacuating the cell containing solid anhydrous oxalic acid and slowly waming the cell up to $100^{\circ} \mathrm{C}$. In this experiment the $\mathrm{KBr}$ windows were kept at $5^{\circ} \mathrm{C}$ higher than the coll body. It was found that

(a) the background spectrum caused by decomposition products is very high and thus some of the gaseous oxalic acid bands are undetectable;

(b) the spectrum also shows that there is a thin film of solid oxalic acid on both $\mathrm{KBr}$ windows. This suggested that the cell temperature is not really lower than the windows. In fact the cell temperature was measured on the cell body and it is possible that the temperature inside the cell is higher than the outside. Spactrum recorded above suggested that

(i) elinination of decomposition products is necessary;

(ii) temperature difference between the cell body and the windows should be higher than $5^{\circ} \mathrm{C}$.

Blimination of the decomposition products was accomplished by connecting the heated cell to the vacum pump and letting the pump operate continuously. In this way it was found that two advantages follow: firstly, the lighter molecules such as $\mathrm{H}_{2} \mathrm{O}$, $\mathrm{CO}_{2}, \mathrm{CO}$ and formic acid which are decomposition products will be pumped out much more quickly than oxalic acid molecules and this eliminatos interference by them; secondly, the cell can be heated up to as high a temperature as one wants without an explosion taking place. 
In order to increase the transmittance of tho beam and also reduce the condensation problem, one $\mathrm{KBr}$ window was replaced by a concave mirror of radius of curvature $33.7 \mathrm{~cm}$ as shown in Fig. 29 below.

The concave mirror is held over a viton rubber ' $O$ ' ring by means of screw-on cap with an electric heater attached. With this set-up the experinent was performed again. The anhydrous oxalic acid was heated up to about $125^{\circ} \mathrm{C}$ while the concave wirror and the $\mathrm{KBr}$ window were heated up to approxinately $10^{\circ} \mathrm{C}$ higher than the cell body. Several spectra of geseous oxalic acid were recorded - see figures $34-47$. Although gaseous oxalic acid bends can be interpreted, it was found that at high temperatures tho spectrum was interferred by solid oxalic acid.

The final experiments were performed recontly with the sane set-uip as above. The rosults are shom in figures $48-51$. This time the anhydrous oxalic acid was purified by sublimation at approxinately $145^{\circ} \mathrm{C}$ before placing in the heated cell. From the spectra recorded the following results vere obtained:-

(1) Sono gascous oxalic acid bands begin to appear at approxinarely $70^{\circ} \mathrm{C}$.

(2) the interference by solid oxalic acid or decomposition products except that of potassium oxalate.

(3) The rate of increase of potassium oxalate increased with temeratures.

(4) Oxalic acid bands aro oasily identified. 
The purification of the oxalic acid before heating in the infrared cell seens to decrease the rate of decomposition.

19.4 Infrared results and the assignment

Spectrum of cold cell

Figures 30 and 31 show spectra of the cold cell (without KBr windows). Several atmosphoric bands were observed as shom in Tablo 16 below.

\section{Table 16}

\begin{tabular}{|c|c|}
\hline $\begin{array}{c}\text { Band position } \\
\left(\mathrm{cm}^{-1}\right)\end{array}$ & $\begin{array}{l}\text { Assignment } \\
\text { (Herzberg 2) }\end{array}$ \\
\hline $3500-4000$ & rotational-vibration $\mathrm{H}_{2} \mathrm{O}$ \\
\hline 3720 & $v_{1}+v_{3} \quad c_{2}$ \\
\hline 3605 & $2 v_{2}+v_{3}-\infty_{2}$ \\
\hline 2340 & $\mathrm{CO}_{2}$ \\
\hline $1300-2000$ & rotational-vibration $\mathrm{H}_{2} \mathrm{O}$ \\
\hline 667 & $v_{2} \quad \mathrm{CO}_{2}$ \\
\hline $500-250$ & pure rotation $\mathrm{H}_{2} \mathrm{O}$ \\
\hline
\end{tabular}

This problem can be eliminated by evacuating the cell. Figures 32 and 33 show spectre of cold cell (with mirror at one end) after evacuating. It is seen that air bands are not detected. Spectrum of gassous oxalic acid

Figures 34 to 51 show spectra of gaseous oxalic acid which were recorded under conditions in Table 17 below. 
Tablo 17

\begin{tabular}{|c|c|c|c|c|}
\hline \multirow{2}{*}{ Fig. No. } & \multicolumn{3}{|c|}{ Tenporature ${ }^{\circ} \mathrm{C}$} & \multirow{2}{*}{ Notes } \\
\hline & Mirror & Windois & $\operatorname{col1}$ & \\
\hline $34: 35$ & 126 & 115 & 93 & $\begin{array}{l}\text { At the end of the run it was } \\
\text { found that the vacuum valve } \\
\text { was blocked by solid oxalic } \\
\text { acid. }\end{array}$ \\
\hline $36 \& 37$ & 101 & 98 & 92 & \\
\hline $38 \& 39$ & 108.5 & 105 & 101 & \\
\hline $40 \& 41$ & 125 & 118.5 & 115 & \\
\hline $42 \& 43$ & - & - & - & $\begin{array}{l}\text { was taken during the cooling } \\
\text { process. }\end{array}$ \\
\hline $44 \& 45$ & - & - & - & $\begin{array}{l}\text { show spectrum of cold cell } \\
\text { left over night (after the } \\
\text { ghove erperiment). }\end{array}$ \\
\hline $46 \& 47$ & - & - & - & $\begin{array}{l}\text { show spectrum of cold cell } \\
\text { (related to } 44 \text { \& } 45 \text { ) aftor } \\
\text { gaseous impurities were punped } \\
\text { out. }\end{array}$ \\
\hline $48 \& 49$ & 123 & 120 & 115 & $\begin{array}{l}\text { The oxalic acid was purified } \\
\text { bofore the experinent was } \\
\text { performed. }\end{array}$ \\
\hline $50: 51$ & - & - & - & $\begin{array}{l}\text { Spectrum of potassium oxrlate } \\
\text { (rolated to Figs. } 48 \text { \& } 49 \text { ). }\end{array}$ \\
\hline $52 \approx 53$ & - & - & - & $\mathrm{KHC}_{2} \mathrm{O}_{4} \cdot \mathrm{H}_{2} \mathrm{O}+\mathrm{NujOI}$ \\
\hline
\end{tabular}

Anhydrous oxalic acid was placed in tho heated cell which was pumped continuous $1 y$. With the optical systen shown in Figs. 27 and 29 the transmittance of the sample beam after passing througin the sample cell was 301. An attenurtor was used in the referenco 
beam so that the pen roved to ebout 80 transmittance at $4000 \mathrm{~cm}^{-1}$.

Figures 36 and 35 showed one of the best spectre of gaseous oxalic acid including inpurities. It was found at the end of this run that the vacuun valve was blocked by solid oxalic acid. This happened because the vacuun valve was cooler than the rest of the cell. It is noticed that in these spectra the fomic acid bands are quite strong.

In ordor to prevent condensation taking place a new vacuum valve was made with en olectric heater acteched to it. Spectra in Figures $36-47$ were taken in the sans experiment at different temperatures. Figures $36-41$ showed spectra of gaseous oxalic acid at approxinately $92^{\circ} \mathrm{C}, 101^{\circ} \mathrm{C}$, and $115^{\circ} \mathrm{C}$ respectively. Figures 42 and 43 ware taken during the cooling process to observe the bohaviour of all bands. It was noticed that the intensities of gasoous oxalic acid bands increased rapidly as the tenperature increased and also rapidy decreasod during the cooling process.

Figures 44 and 45 woro taken after the cell was left over night with vacuus valve closed. The spectrum of the cold cell left over night showed no sign of gaseous oxelic acid bands and only that of impurities and a thin film of solid oxalic acid. Figures 46 end 47 show spectre of a thin filn of solid oxalic acid on the KBr window. It was taken after the impurities in the cold coll were pumbed away.

Figuros 48 and 49 showed spectra of the final experiment in which the oxalic acid was purified before placing into the heated cell. Figures 50 and 51 showed the spectra of potassium oxalate 
forming during the experinent. These spectro were taken after the cell was cooled down and the gaseous lmpuritios had been purmed anay.

Fron the spectra mentioned above, 27 bands were observed, 8 were assigned to gasoous axalic acid, the others were assigned to inpuritios as show in Tablo 18 below. The spectrum number in the table shous the bost example of the band. Many bands appear in spectra.

Table 18

\begin{tabular}{|c|c|c|c|}
\hline $\begin{array}{l}\text { Band position } \\
\qquad\left(\mathrm{cm}^{-2}\right)\end{array}$ & $\begin{array}{l}\text { Spectrum } \\
\text { Number }\end{array}$ & Intensities & Assigned to \\
\hline 3720 & 34 & & $\infty_{2}$ \\
\hline 3560 & 34 & $w$ & gaseous oxalic acid \\
\hline 3475 & 34 & v.s. & gaseous oxalic acid \\
\hline 2960 & 34 & & formic acid \\
\hline 2340 & 34 & & $\mathrm{CO}_{2}$ \\
\hline $\int 1820$ & 34 & v.s. & gaseous oxalic acid \\
\hline 1805 & & & \\
\hline $1760 \int^{1770}$ & 34 & & formic acid \\
\hline 1730 & 46 & & solid oxalic acid ( $B$-form) \\
\hline 1720 & 50 & & potassium hyàrogen oxalate \\
\hline 1.620 & 50 & & potassium hydrogen oxalate \\
\hline 1400 & 51 & & potassium hydrogen oxalate \\
\hline
\end{tabular}




\begin{tabular}{|c|c|c|c|}
\hline $\begin{array}{l}\text { Band position } \\
\qquad\left(\mathrm{cmi}^{-1}\right)\end{array}$ & $\begin{array}{l}\text { Spectrun } \\
\text { Number }\end{array}$ & Intensitios & Assigned to \\
\hline 1325 & 39 & v.s. & gastous oxalic acid \\
\hline$(1285$ & & & \\
\hline $1275\{1277$ & 39 & v.s. & gaseous oxallic acid \\
\hline 1.265 & & & \\
\hline 1280 & 51 & & potassium hydrogen oxalate \\
\hline $1265^{\circ}$ & 45 & $\ddots$ & calnown impurity \\
\hline 1225 & 47 & & solid oxalic acid ( $\beta$-form) \\
\hline 1215 & 51 & & potassium hydrogen oxalate \\
\hline $\int 1115$ & & & \\
\hline$1 1 0 0 \longdiv { 1 1 0 3 }$ & 35 & 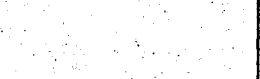 & formic acid \\
\hline 1085 & & & \\
\hline $1030^{*}$ & 43 & & unknom inpurity \\
\hline $815^{*}$ & 43 & & uznom impurity \\
\hline 720 & 51 & & potassium hydrogen oxalate \\
\hline 700 & 47 & & solid oxalic acid ( $\beta$-form) \\
\hline 667 & 45 & & $\mathrm{CO}_{2}$ \\
\hline 650 & 41 & $s$ & gaseous oxalic acid \\
\hline 620 & 41 & $y$ & possibly gaseous oxalic acid \\
\hline 495 & 51 & & potassium hydrogen oxalate \\
\hline 460 & 41 & w & gassous oxalic acid \\
\hline
\end{tabular}

V.S. very strong, $s$ strong, w weak

- They are definitoly impurity bands because they exist in the spectra of the cold ceil Ieft after the experiment. 
WOTE: The supporting references or spectra for impurity bands are in $(62),(128)$ and $(129)$.

For comparison, the spectrum of potassium hydrogen oxalato monohydrate, $\mathrm{MHC}_{2} \mathrm{O}_{4} \cdot \mathrm{H}_{2} \mathrm{O}$, in nujol mull is given in figores 52 and 53. 


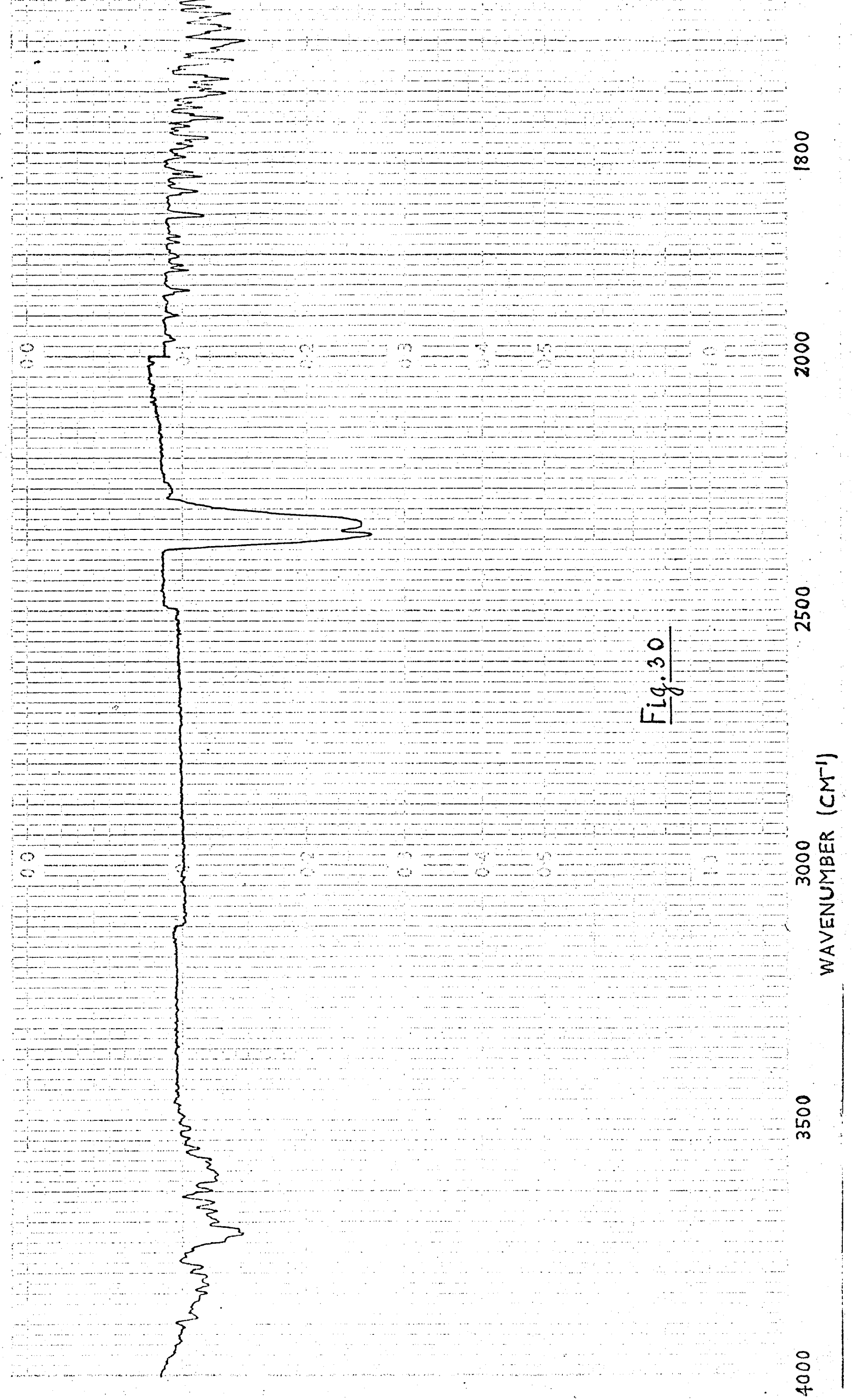




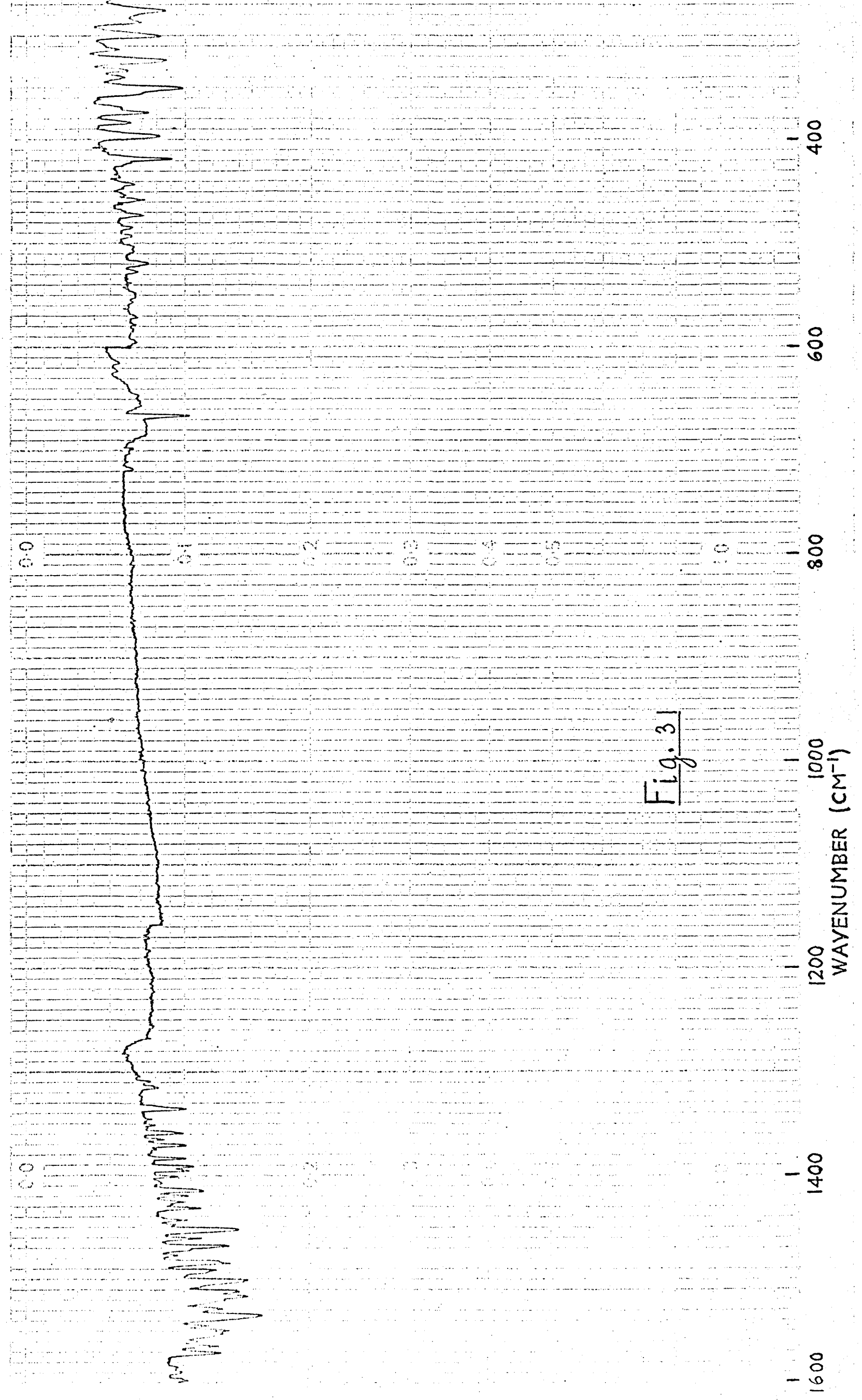




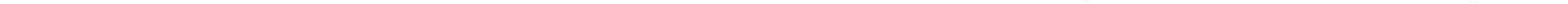




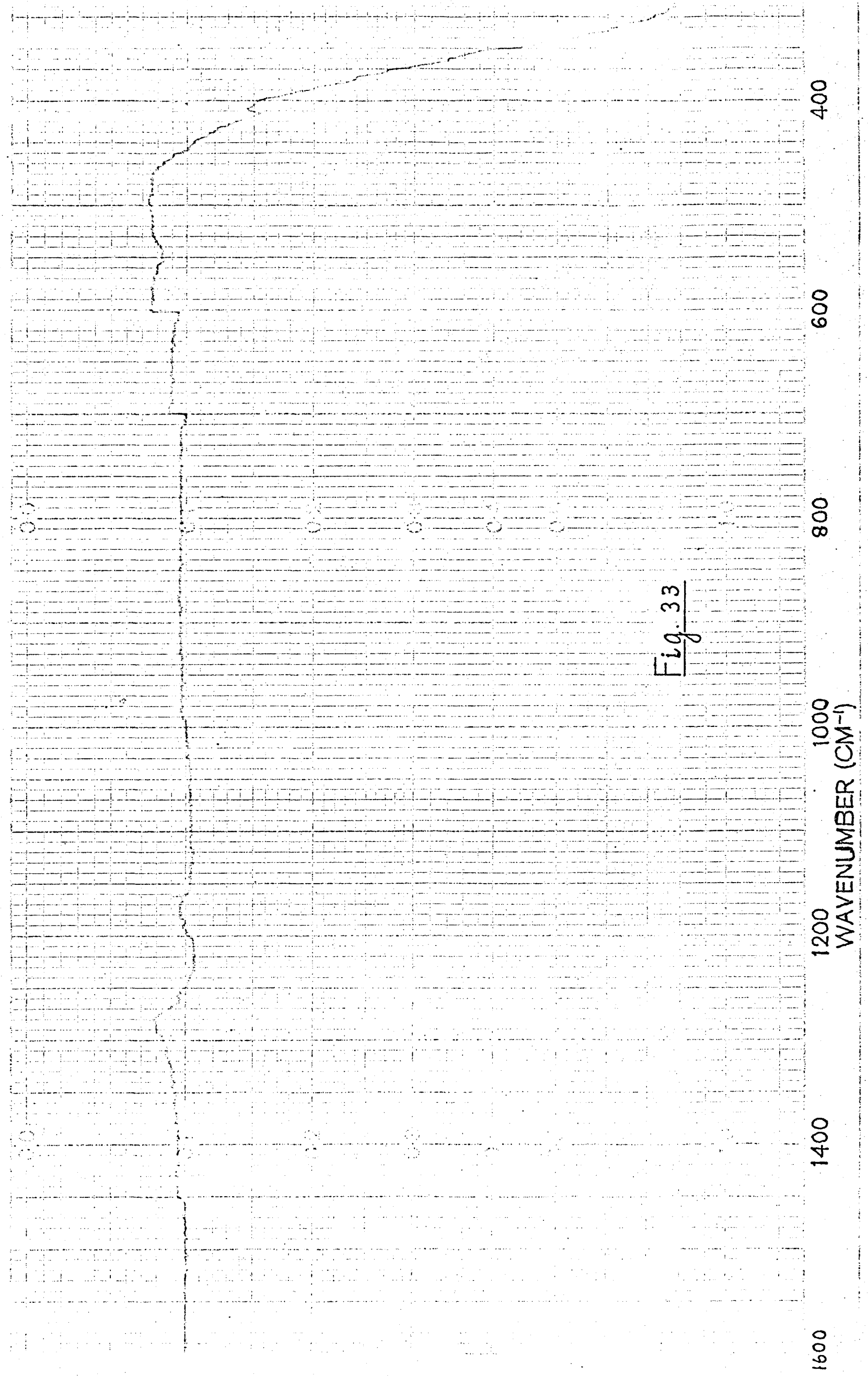




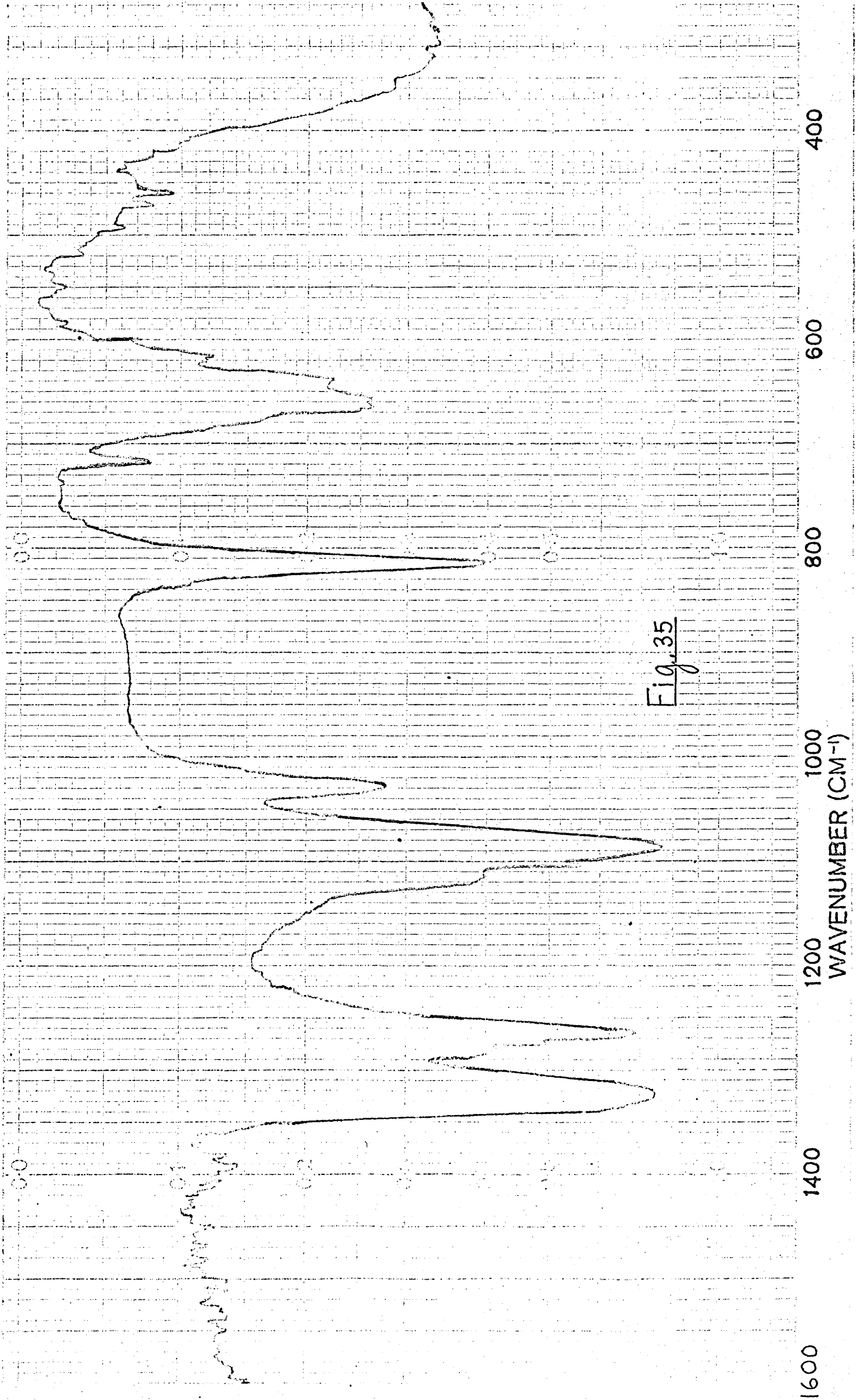




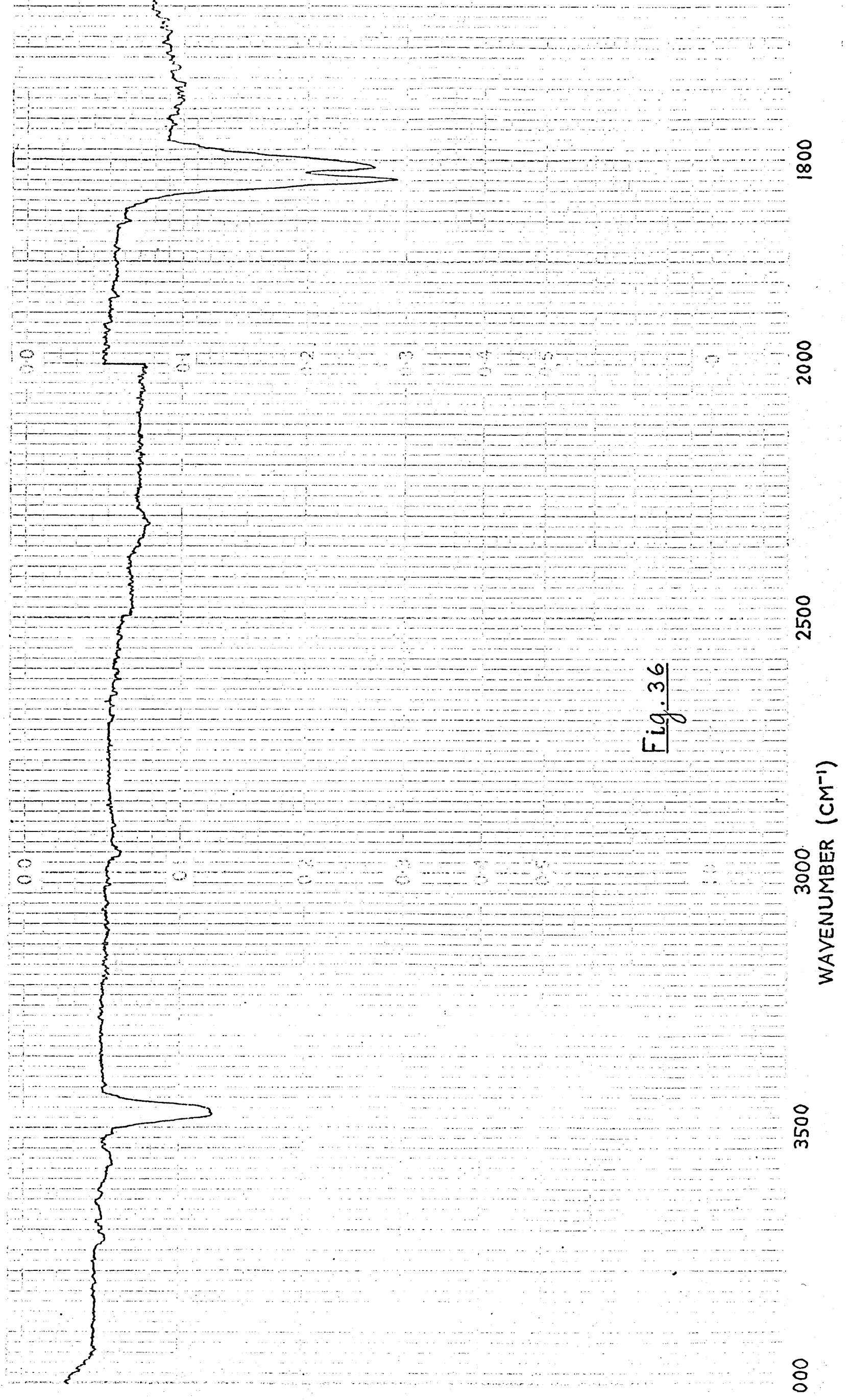




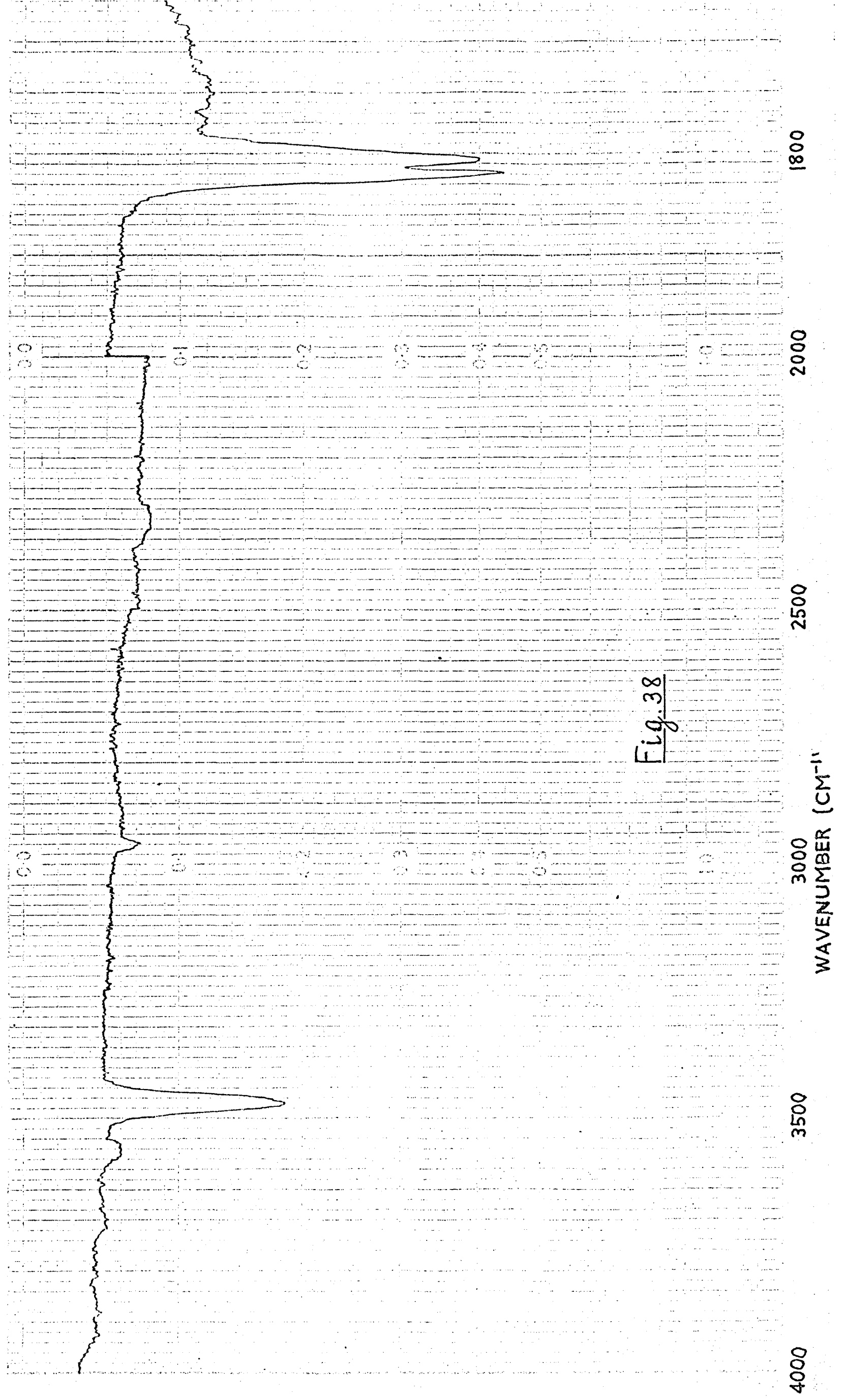




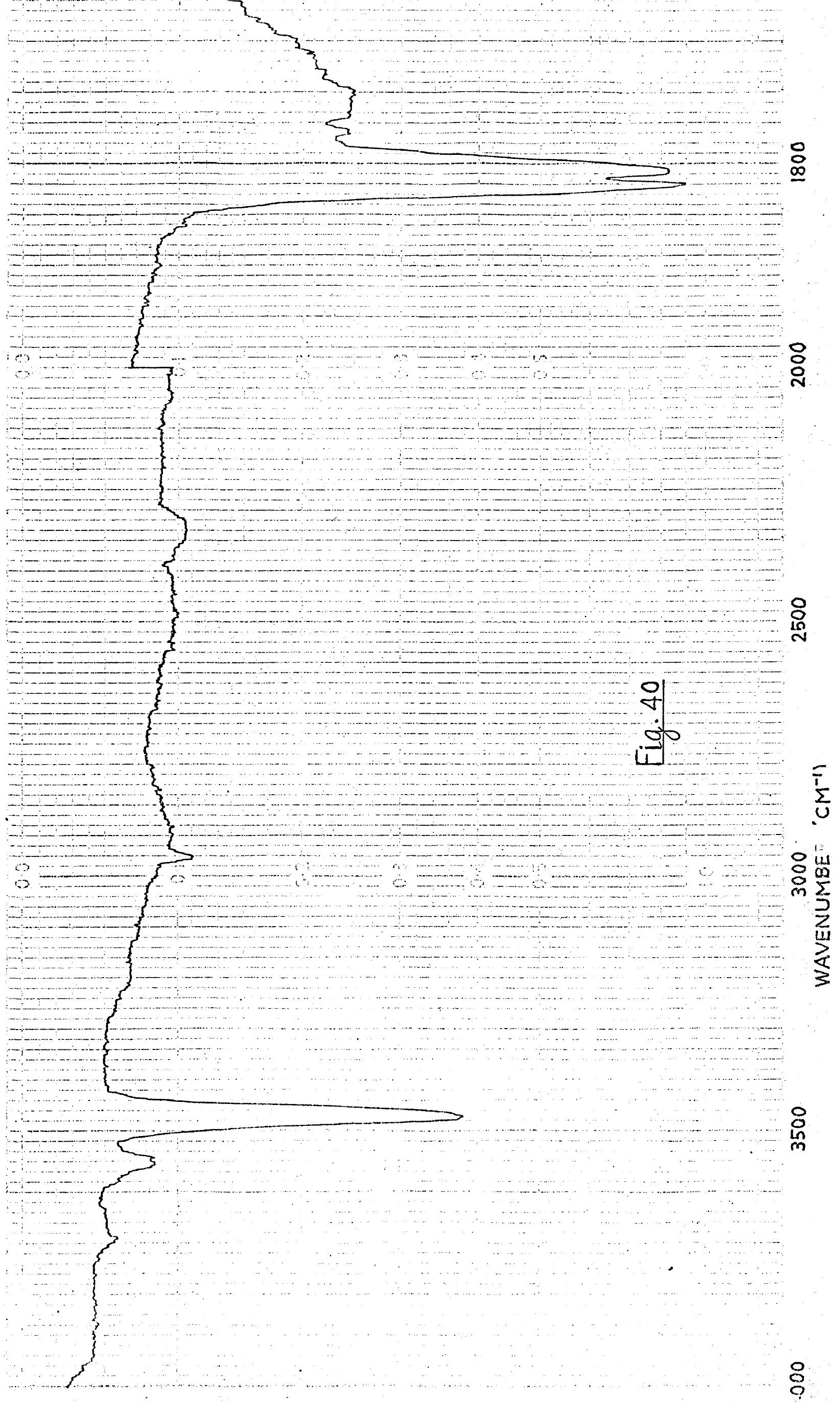




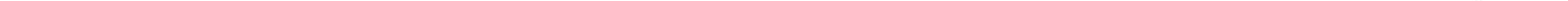




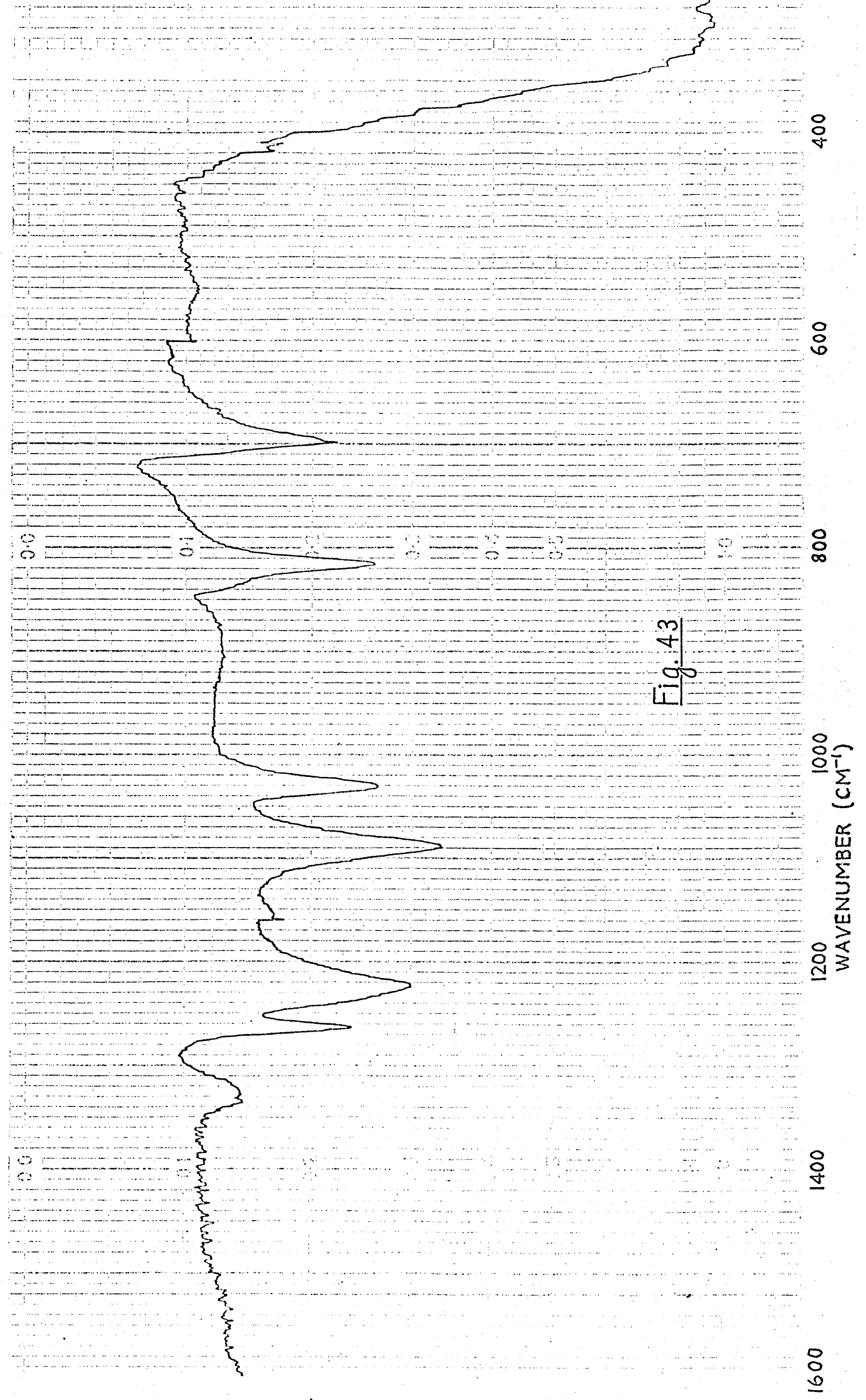




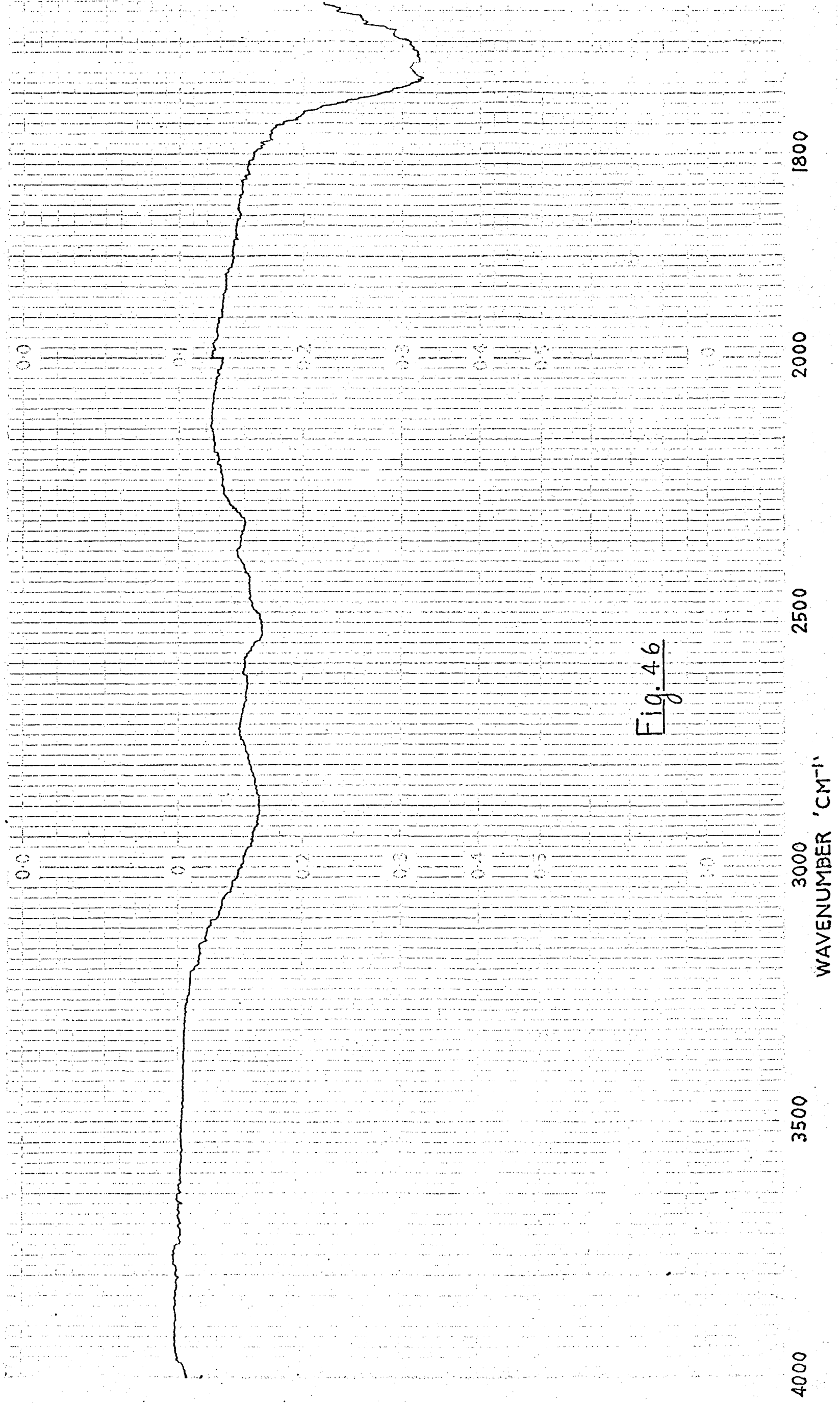




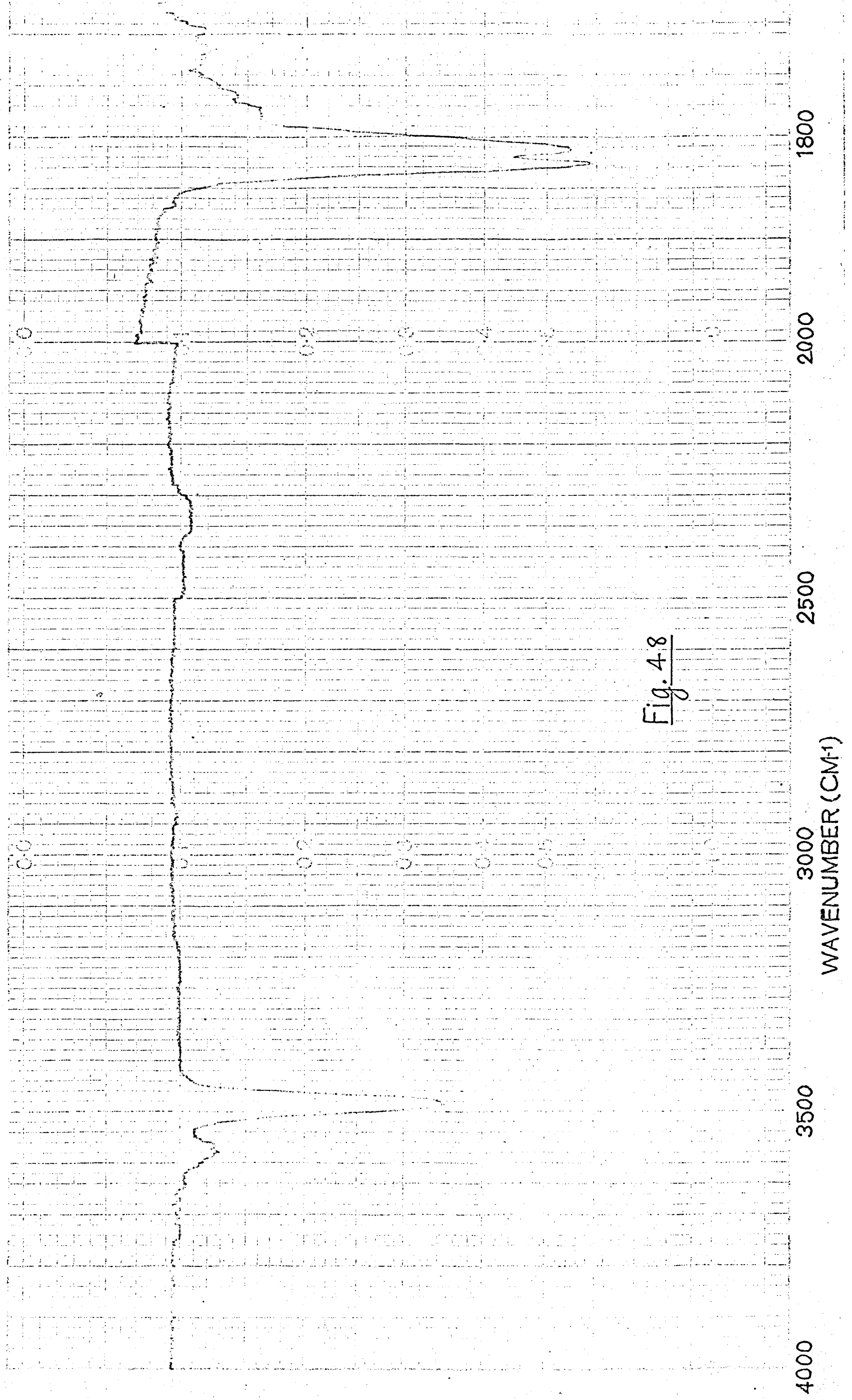




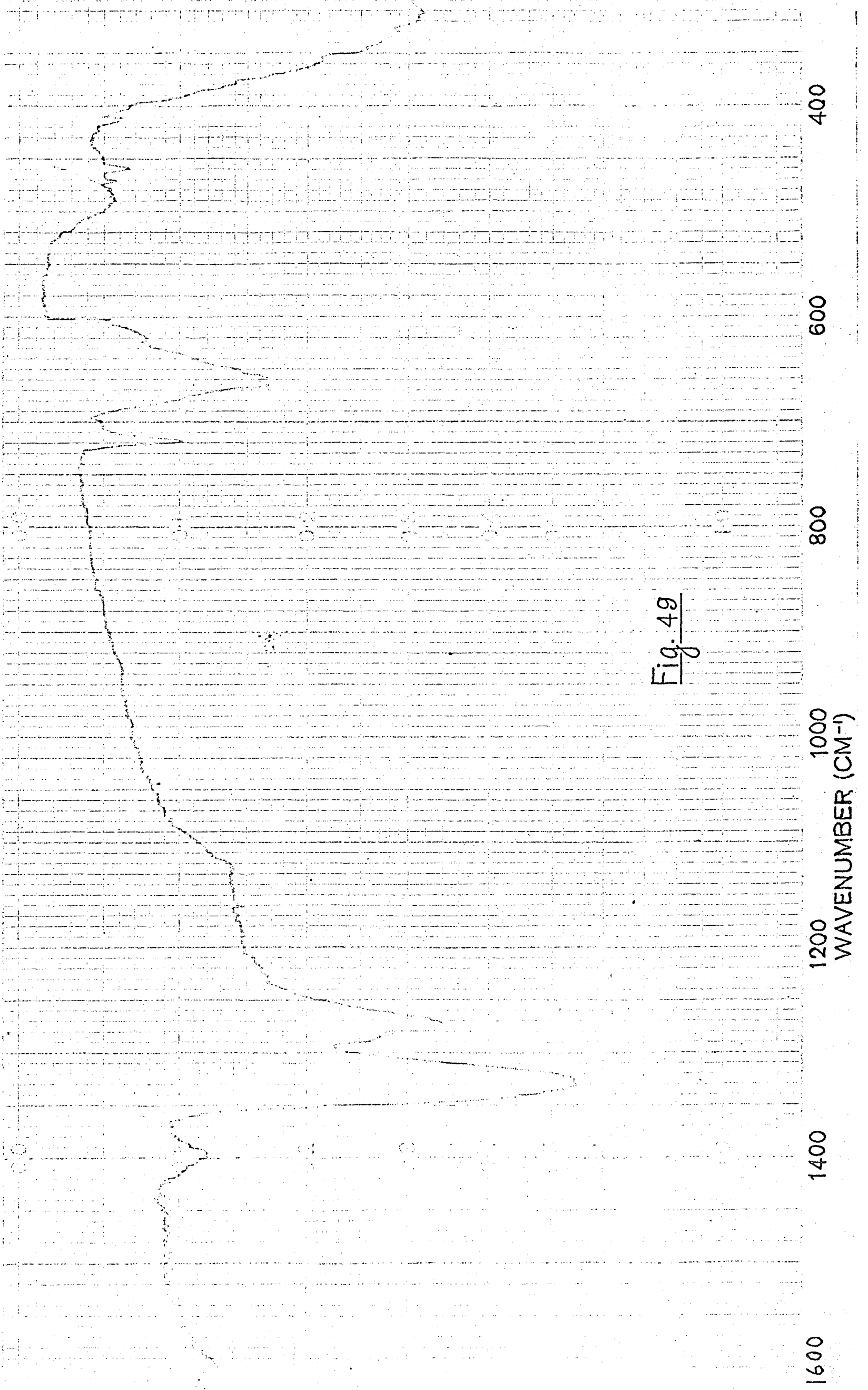




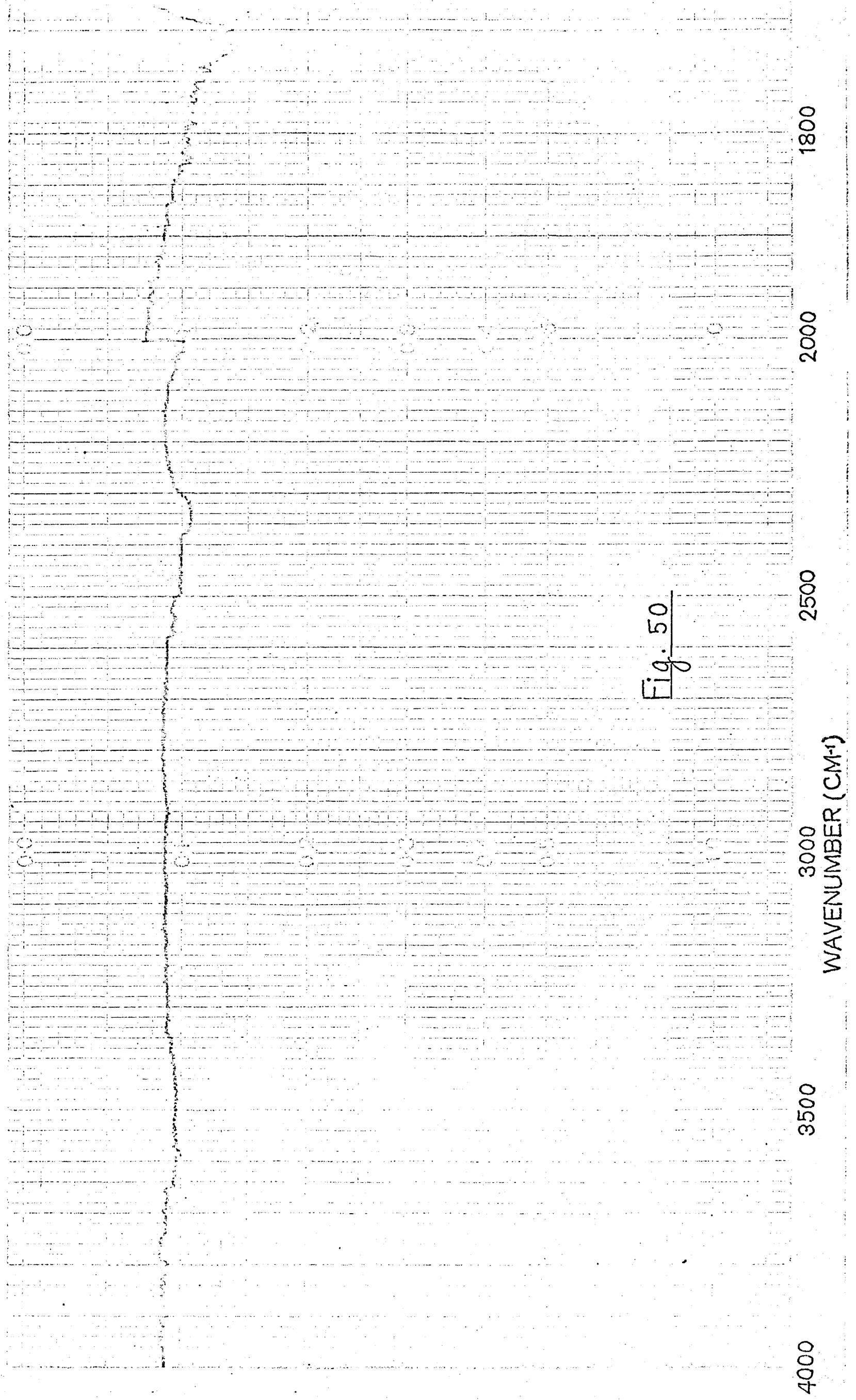




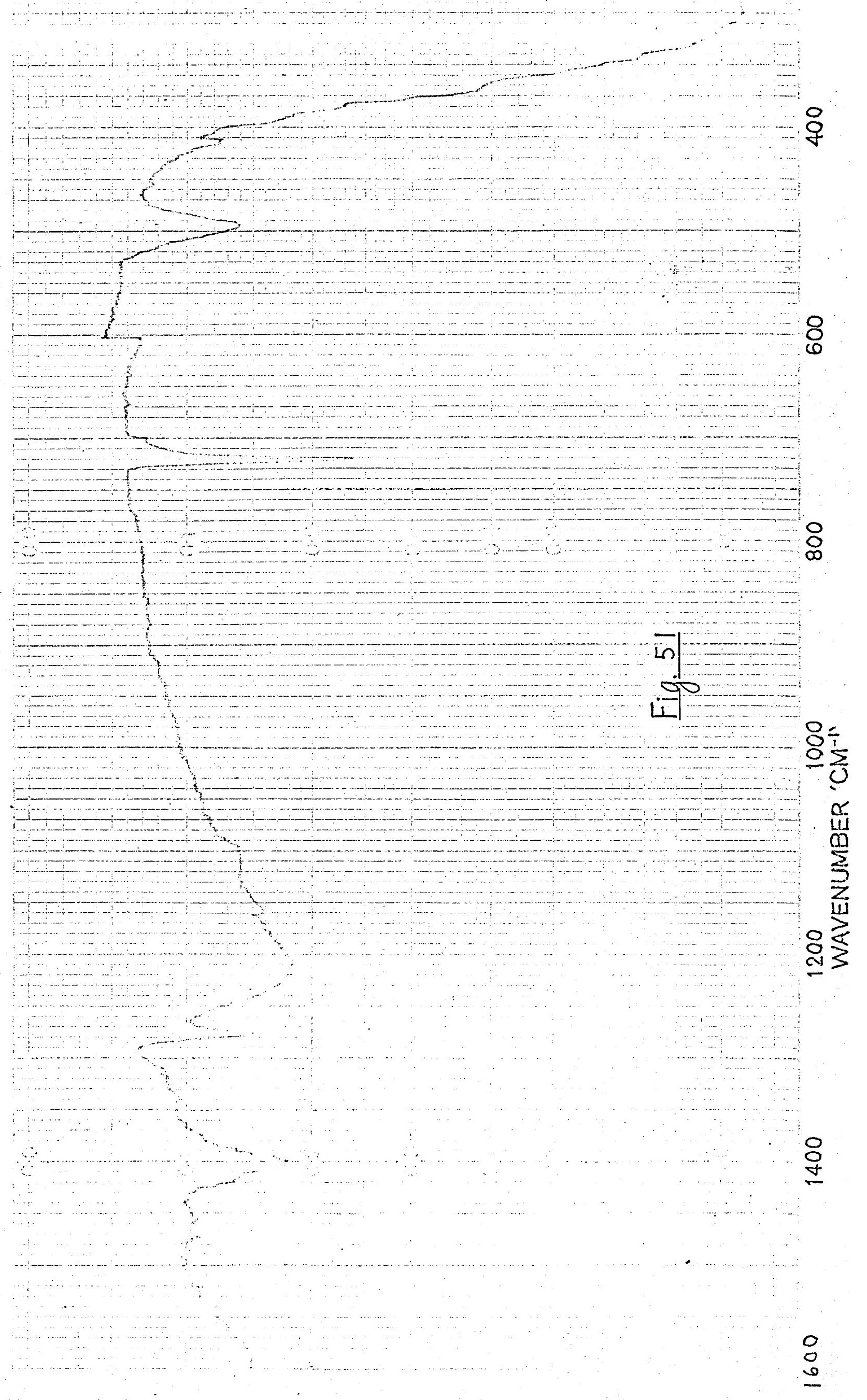




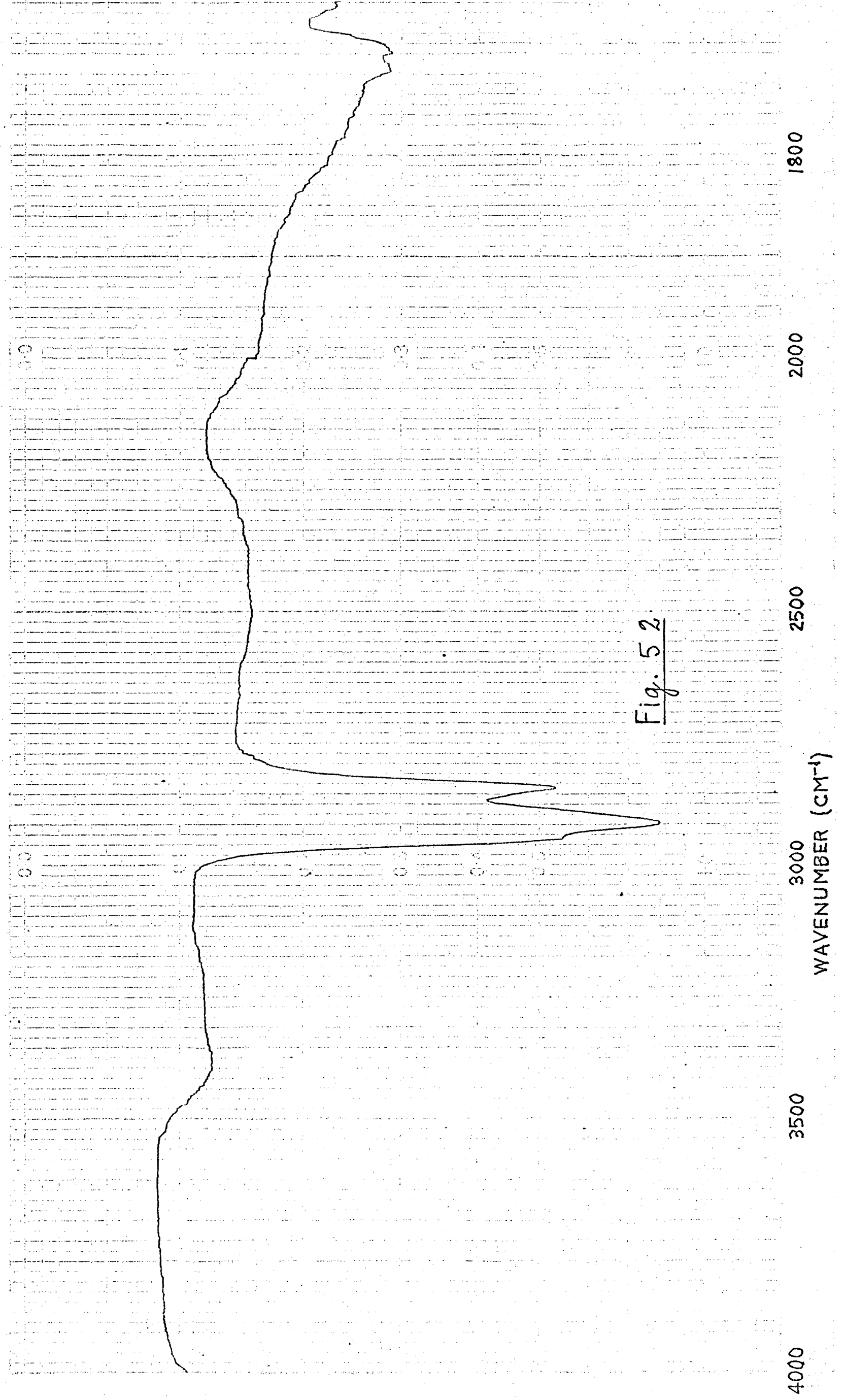


Tho assignment of the gaseous oxalic acid bands

The assigament of gascous oxalic acid bands was made on the basis that: $\rightarrow$

(1) Gascous oxalic acid bands aro expected to be observed at terperatures above $60^{\circ} \mathrm{C}$ with their intensities varying with teraperatures.

(2) Impurity bands are still observed in the spectrun of the cooled cell but not gaseous oxalic acid bands.

(3) It is assumad that fundarantals are stronger than overtones and combinations.

(4) In the infrared, intensities of the out of plane vibrational bands are usually stronger than the inplane vibrations (119).

(5) For gaseous oxalic acid it is not expected to see any out of plane fundaraentals above $1100 \mathrm{~cm}^{-1}$ (109), (4).

(6) Because of Fermi resonance, two close bands of the sano species interect with each other. Thus, if two bends colncide or overlap they should belong to different species.

(7) Gaseous oxalic acid band shapes are different from impurities because of the different moments of inertia.

As shown in Table 18, the bands at $3560 \mathrm{~cm}^{-1}, 3475 \mathrm{~cm}^{-1}, 1812 \mathrm{~cm}^{-1}$, $1325 \mathrm{~cm}^{-1}, 1275 \mathrm{~cm}^{-1}, 660 \mathrm{~cm}^{-1}, 620 \mathrm{~cm}^{-1}$, and $460 \mathrm{~cm}^{-1}$ were assigned to gaseous oxalic acid. The band at $620 \mathrm{~cm}^{-1}$ is doubtful because it is weak and ovorlapped with the $660 \mathrm{~cm}^{-1}$ band. Assignment of gaseous oxalic ncid bands is shown in Table 19 below. 
Table 19

Gascous oxalic acid bands and their assigmments

\begin{tabular}{|c|lc|cc|}
\hline \multirow{2}{*}{$\begin{array}{c}\text { Band position } \\
\left(\mathrm{cm}^{-1}\right)\end{array}$} & \multicolumn{4}{|c|}{ Assignment } \\
\cline { 2 - 5 } & \multicolumn{3}{|c|}{ 1st node1 } & \multicolumn{2}{c|}{ 2nd node1 } \\
\hline 3560 & $v_{2}+v_{8}$ & $\mathrm{Bu}$ & $v_{2}+v_{8}$ & $\mathrm{Bu}$ \\
3475 & $v_{10}$ & $\mathrm{Bu}$ & $v_{10}$ & $\mathrm{Bu}$ \\
1812 & $v_{8}$ & $\mathrm{Bu}$ & $v_{8}$ & $\mathrm{Bu}$ \\
1325 & $v_{9}$ & $\mathrm{Bu}$ & $v_{13}$ & $\mathrm{Bu}$ \\
1275 & $v_{13}$ & $\mathrm{Bu}$ & $v_{9}$ & $\mathrm{Bu}$ \\
660 & $v_{11}$ & $\mathrm{Bu}$ & out of plane $\mathrm{Au}$ \\
620 & out of plane & $\mathrm{Au}$ & $? ?$ & $\mathrm{nu}$ \\
460 & out of plane & $\mathrm{Au}$ & $v_{12}$ & $\mathrm{Bu}$ \\
& & & & \\
\hline
\end{tabular}

Cnly two bands ar $3560 \mathrm{~cm}^{-1}$ and $3475 \mathrm{~cm}^{-1}$ were observed in the 0 -1 asymetric stretching frequency region. The band at $3475 \mathrm{~cm}^{-1}$ is much stronger and thus it was assigned to fundamontal $v_{10}$, asynuotric $0-11$ stretch, Bu species. The band at $3560 \mathrm{~cm}^{-1}$ was then assigned to conblnation band, $v_{2} * v_{8}$, Bu species. $\left(v_{2}=1800 \mathrm{~cm}^{-1}\right.$, seo section 20.4$)$.

The strong band of $1812 \mathrm{~cm}^{-1}$ has two band heads at $1820 \mathrm{~cm}^{-1}$ and $1805 \mathrm{~cm}^{-1}$ respectively. This band was assigned to the inpiane fundamental $v_{8}$, asymatric Cou strotch, Bu species, because it is the only band in the carbonyl stretching froquency range.

Two strong bands at $1325 \mathrm{~cm}^{-1}$ and $1275 \mathrm{~cm}^{-1}$ wore assigned to the interaction of the inplane C-O asymetric stretch $v_{9}$ and the 
inplane C-0-1t angle bending wole $v_{13}$ (4). The best fit to the calculations is obtainod by revorsing the assigntant for the two rodels.

Assignnents for three bands at $660 \mathrm{~cm}^{-1}, 620 \mathrm{~cm}^{-1}$, and $460 \mathrm{~cm}^{-1}$ were made to fit the calculated frequoncies. Thus, thoir assignnents were different for both models. The band at $660 \mathrm{~cm}^{-1}$ was assigned to inplane fundamantal $v_{11},-C \leqslant_{0}^{0}$ angle deformation and the other two were assigned to out of plane fundarentals for the first nodel. For the second rodel the band at $460 \mathrm{~cm}^{-1}$ was assigned to inplane fundamentals $v_{12},(0 . . \mathrm{H}-0)$ angle deformation. The band at $660 \mathrm{~cm}^{-1}$ was assigned to the out of plane fundernontal and the band at $620 \mathrm{~cm}^{-1}$ could be assigned to conbination band, but see however chapter 22.

10.5 The expected and observed band envelopes of geseous oxalic acid

As nentioned previously in section 11.2 , the theoretical band envelopes and the $P R$ separations for asymetric top rolecules have been studied by many authors $(26),(55),(69),(71)$, (112), (125), (126).

Artong then the paper which was published by lede and Shimanouchi ssems to be the most useful set. Thus the expected band contours for geseous oxalic acid were taken from this paper. The molecular paraneters of gaseous oxalic actd are given in Table 20 belor. 
Table 20

Molecular parameters for gaseous oxalic acid

\begin{tabular}{|c|c|c|}
\hline & First model & Second mode1 \\
\hline Molecular model & & \\
\hline $\begin{array}{l}\text { Principal moments } \\
\text { of inertia } \\
\left(\mathrm{gm} \cdot \mathrm{cm}^{2} \times 10^{40}\right)\end{array}$ & $\begin{array}{l}I_{A}=138.02 \\
I_{B}=239.49 \\
I_{C}=377.51\end{array}$ & $\begin{aligned} I_{A} & =146.71 \\
I_{B} & =221.05 \\
I_{C} & =367.76\end{aligned}$ \\
\hline $\begin{array}{l}\text { Üeda and Shimanouchi } \\
\text { parameters }\end{array}$ & $\begin{aligned} x & =2 C / B=1.269 \\
y & =1-(C / A)-(C / B) \\
& =0\end{aligned}$ & $\begin{array}{l}x=1.202 \\
y=0\end{array}$ \\
\hline
\end{tabular}

Using Ueda and Shimanouchi parameters it is expected that the gaseous oxalic acid will have band contours similar to that given in figure 54 for $A, B$ and $C$ type bands. 


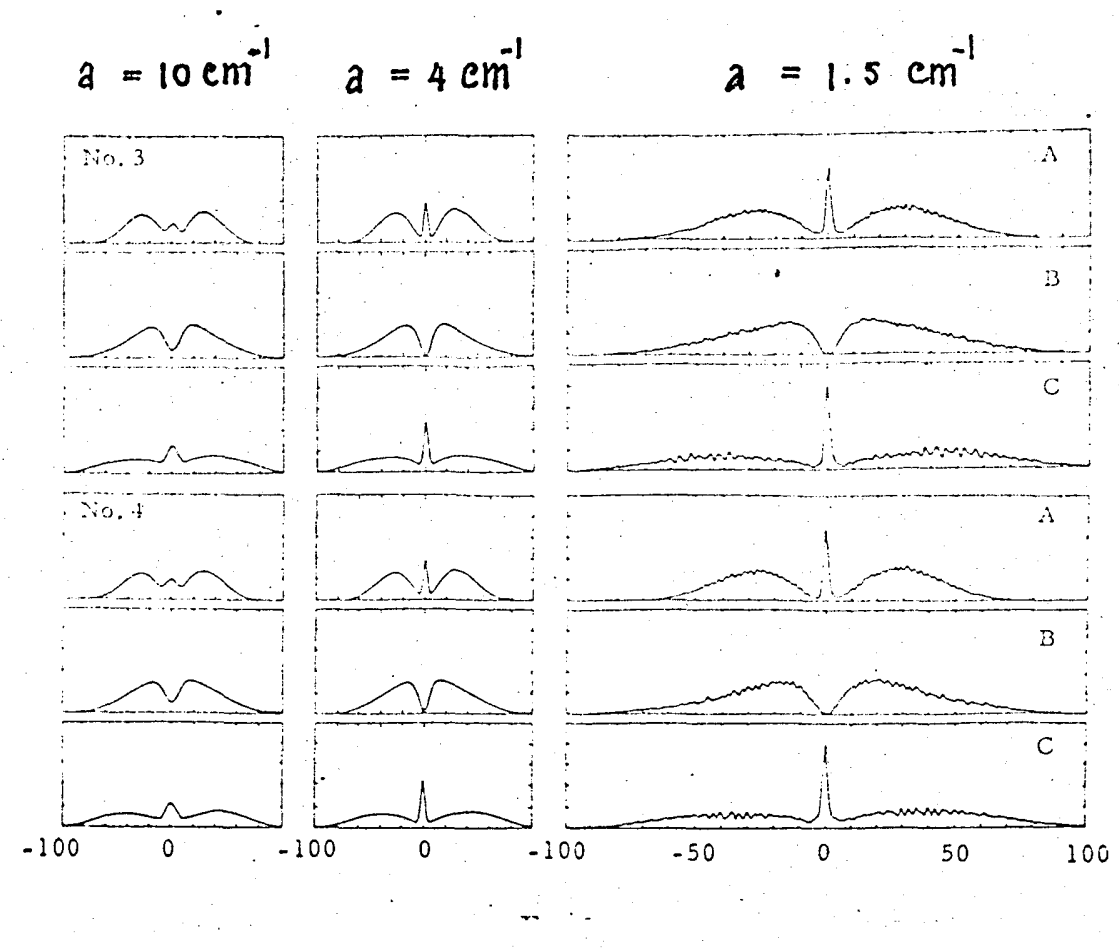

Fig. 54

The expected band contours for gaseous oxalic acid (a is the halfwidth of the slit)

These contours were taken from figure No. 3 and No. 4 in the Ueda and Shimanouchi paper in which the corresponding parameters are given below.

$\begin{array}{cccccc}\text { №. } & \mathrm{x} & \mathrm{y} & \mathrm{A} & \mathrm{B} & \mathrm{C} \\ 3 & 1.2 & 0.0 & 2.419 & 1.613 & 0.968 \\ 4 & 1.3 & 0.0 & 2.648 & 1.426 & 0.927\end{array}$

Both sets of parameters above give similar band envelopes. That is, the $B$ type envelope has no central $Q$ branch. The $A$ and $C$ type envelopes have strong and sharp $Q$ branch. 
At the beginaing it was oxpected that having studied the band contours, the gaseous onalic acid bands could be assigned to $A, B$ and $C$ type bands. Thus, the out of plane vibrations, which are $C$ typs bands, could be picked out. thfortunately, this is not to be bocauso

(1) Both A and $C$ type envelopes are similar and it is difficult to detect the difference botween them.

(2) Most observed band contours of gasoous oxalic acid are not sinilar to the expected band contours mentioned above. This is because most observed band envelopes are nostly of lydrid bands.

Fron the snactra recorded the observed band contours do not help to determine $\mathrm{C}$ type bands nor to distinguish between the two models. Wreover, the vectors diagrams obtained fron the cormuter, chapter 22 , do not allow the typo of hybrid to bo deterninea. Thus there is no reason to get the spectra recorded et higher resulution. 


\section{CHAPTER 20}

RAMAN EXPERTMENT

20.1 Laser Raman instrument

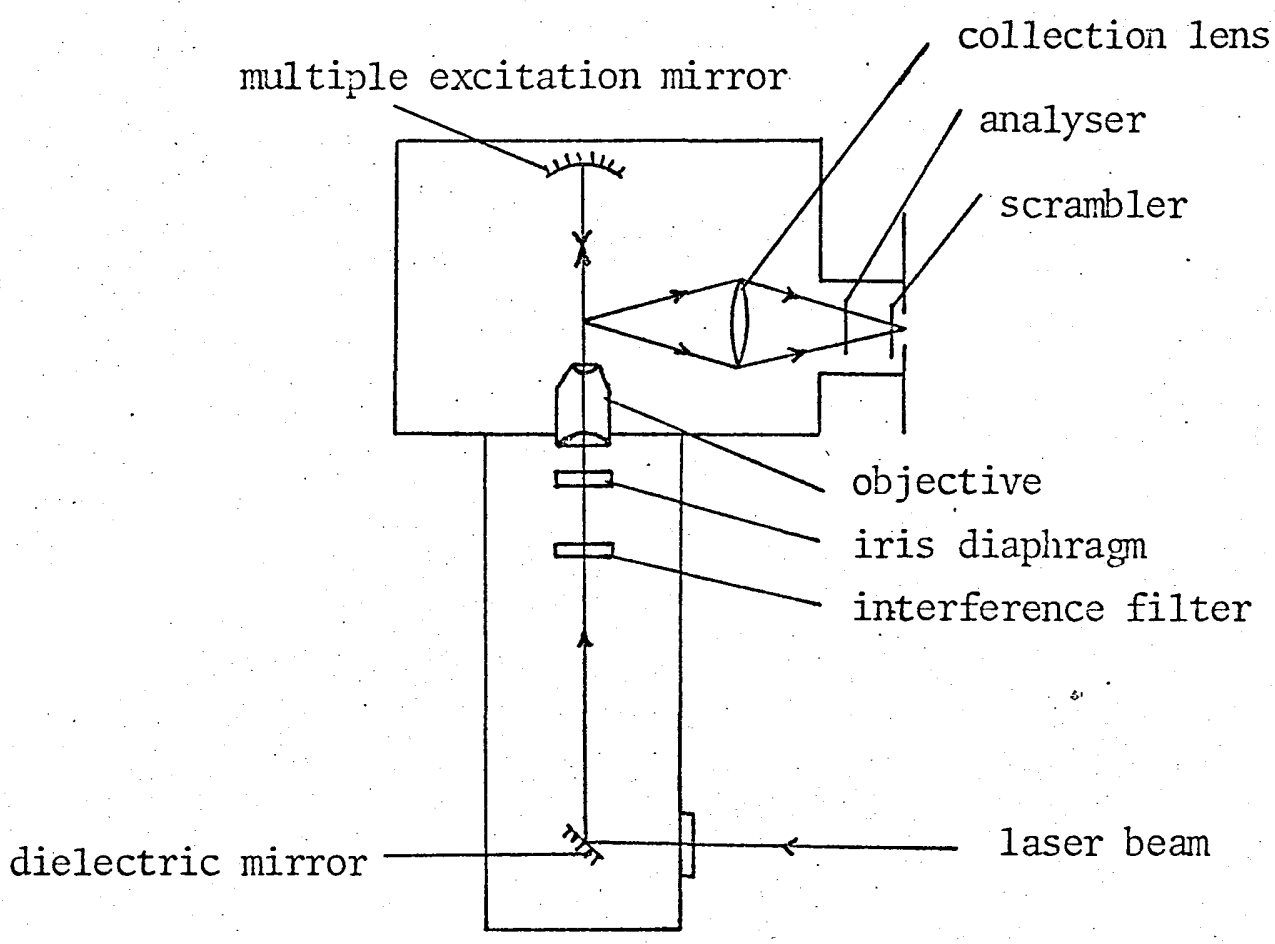

Fig. 55

Sample optics for Spex Raman spectrometer

The Raman instrument used was the Spex 1401 double monochromator with either

(1) Spectra-Physics He - Ne laser mode1 125

(2) Spectra-Physics $\mathrm{Ar}^{+}$laser model 164

As shown in Fig. 55 the laser beam is reflected upwards by

a dielectric mirror in a light tight tube beneath the sample 
chamber. The interference filter is talen into the bean to reduce intorference from non-lasing lines in the discharge (plasme 1ines). An iris dipohrema reluces interference from tho plasma lines and finally a nicroscope objective lens focuses the bean to a diffraction-linited spot in the sanple. Mirror $y_{1}$ serves to Increase the excitaticn energy by retuming the laser beam through the sample and the collection lens focuses the scattered light on to the spectrometer entrance slit. The light tight tube (tumel) bridges the illuminator and the spectronetor entrance slit. It contnins the polerizing anelyser and a crystal quartz polarizing scrambler vedge to effectively depolarize the Raman radiation end avid masurenent errors arising from the polarization effects of the gratings.

Fig. 50 shows the coublemonochromator, that is, che monochronator followed by and cowpled to a second. The centres of the entrance slit and exic slit 110 in the optical plane of the

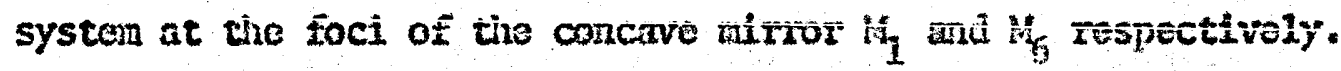
Light coning through the entrance slit is collibated by mirror $M_{1}$ and reflectod onto the grating $G_{1}$. The dispersed light strikes mirror $H_{2}$ and is focused onto mirror $u_{3}$ which reflects the bean through tho slit $S_{2}$ and it hits mirror $\mathrm{H}_{4}$. The reflected light from $\mathrm{N}_{4}$ will be collinated by concave mirror $\mathrm{M}_{\mathrm{S}}$ and reflected onto the grating $G_{2}$. The disporsed ligint strikes the concavo mirror $M_{6}$, which brings tho spectrum to a focus at the exit slit. 


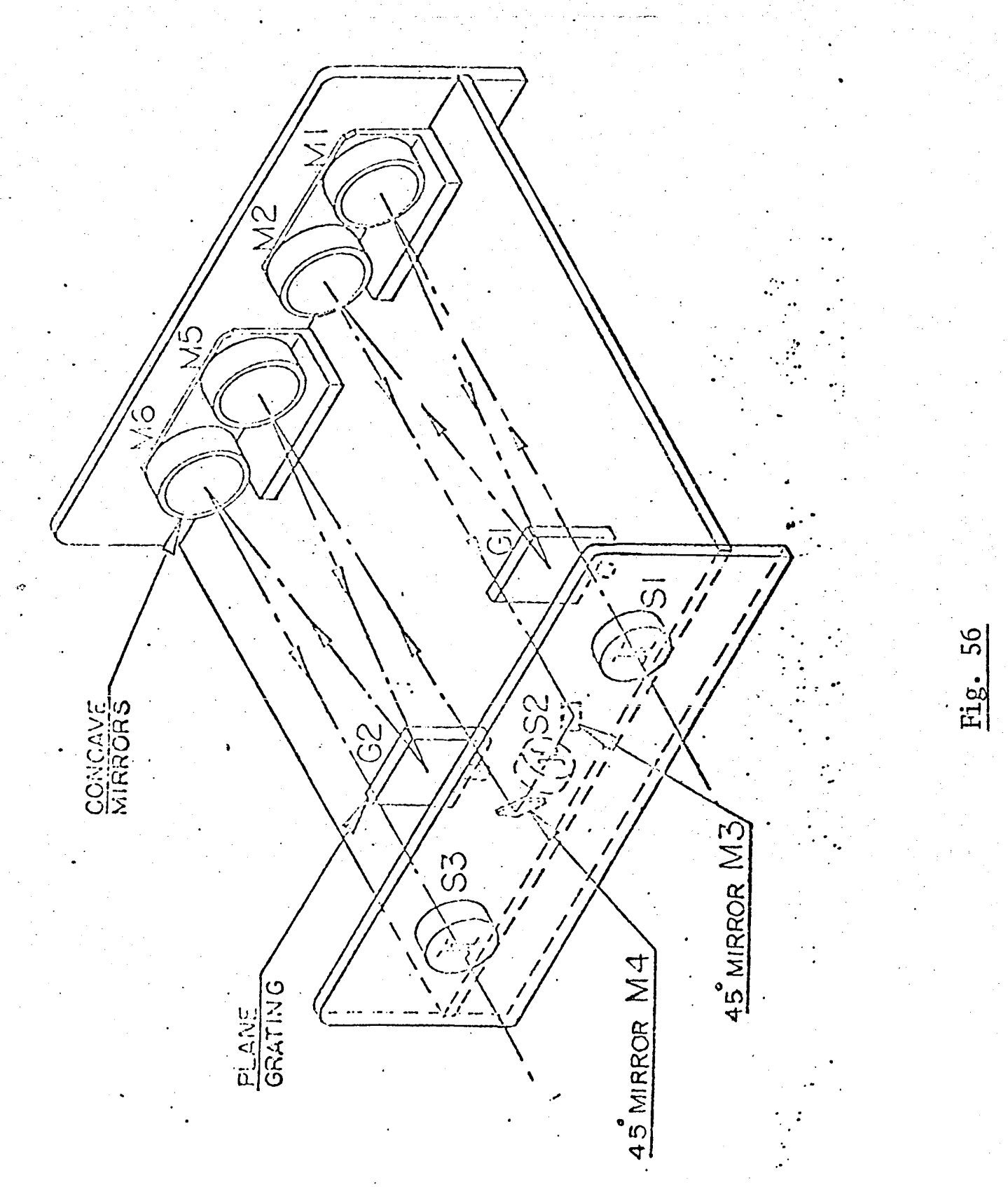


A double monochromator hes the advantage that dispersion at the final slit is toice as mucin as at the intermediate. One more advantage is that in the second part of the double ronochromator the radiation is dispersed, so that tho unanted exciting radiation is rejocted.

The phocomutiplier used in the Rasan experinent is a Fit-130 tube. It was clained that the red sersitivity of the Fi-130 is far better then that of un ordinary S-20 photomutiplier. Another significant advantage in signni detection of the Fit-130 photonultiplier is derived from its 3.5 m effective cathode dinneter as compared vith ordinary tubes having $20-50 \mathrm{~mm}$ dineter cathode. The ms nolse of a cathode surface is proportional to its dianeter.

It was also cleined that the dark current of the rod stusitivity of mit-130 photomiltipliers drons by a factor of epproximately 100 winen the tube is coolea to $-20^{\circ} \mathrm{C}$. The cooler used is a themolectric chanber with tap water at a flow rate of 10 geh serves as a heet exchenger.

\subsection{Raman oven}

A Reman oven was designed to use with the Spox 1401 doublemochromator. Its body is made of aluniniun of cylinarical shape with dimansions shown in the figures below. 


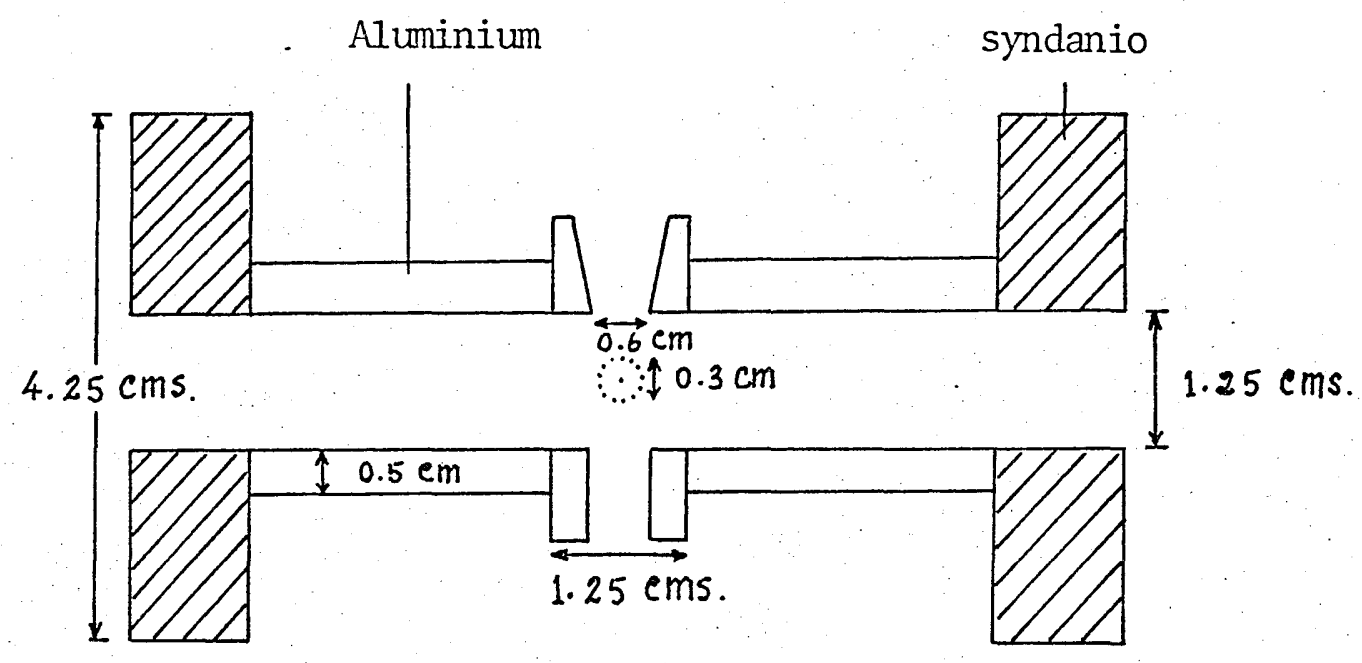

Fig. 57

X-section of Raman oven along the whole length

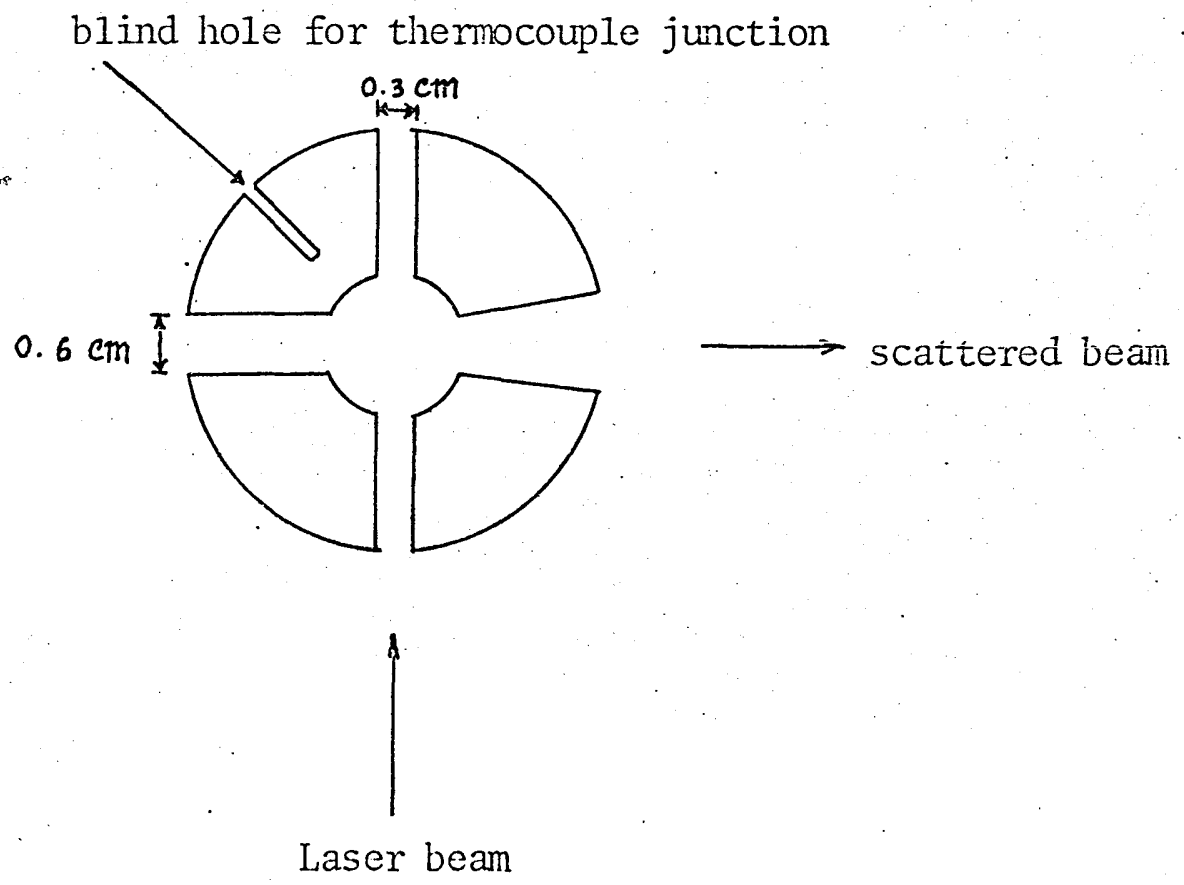

Fig. 58

Side view X-section of the oven at sample position 
Two electrothermal heating wires HC 102 (V200/250 - 120W) were used to heat the oven. These heating wires were insulated with glass fibre. The wattage of these wires can be varied by means of a variac. Both ends of the oven were insulated using Syndanio, the body was insulated using Pyruma. fire cement. The length of the oven was $10 \mathrm{~cm}$ and its inner diameter was $1.25 \mathrm{~cm}$. On the oven body there were holes for the laser beam to pass through, holes for the scattered beam to pass through and a blind hole for a thermocouple junction.

Fig. 60 shows the curve of the equilibrium temperature against the voltage supply of this Raman oven.
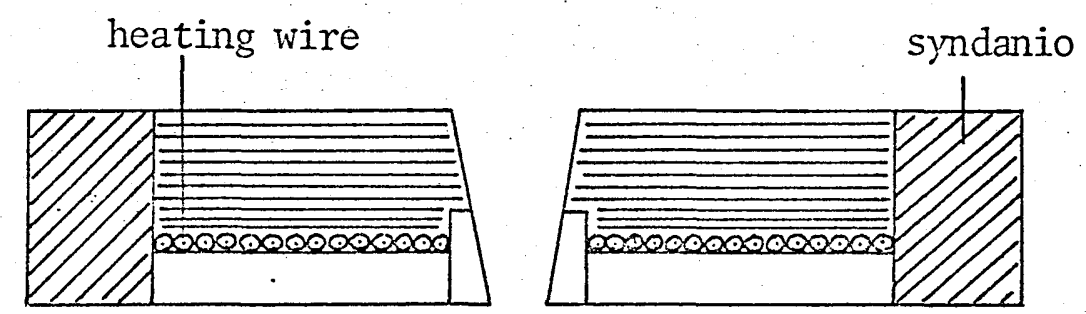

$\because$ Laser beam
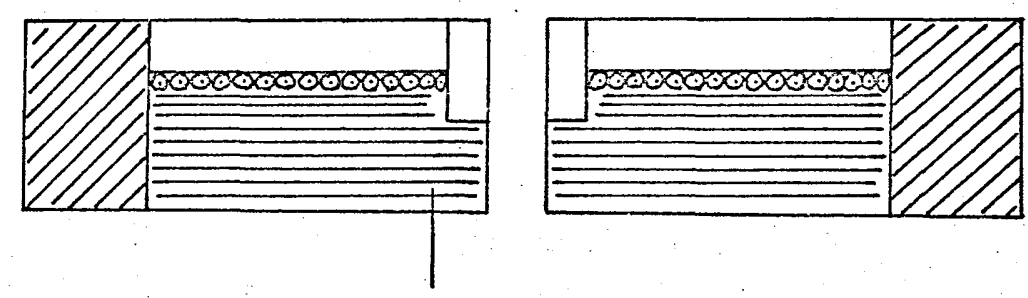

Pyruma fire cement

Fig. 59

The Raman oven used 


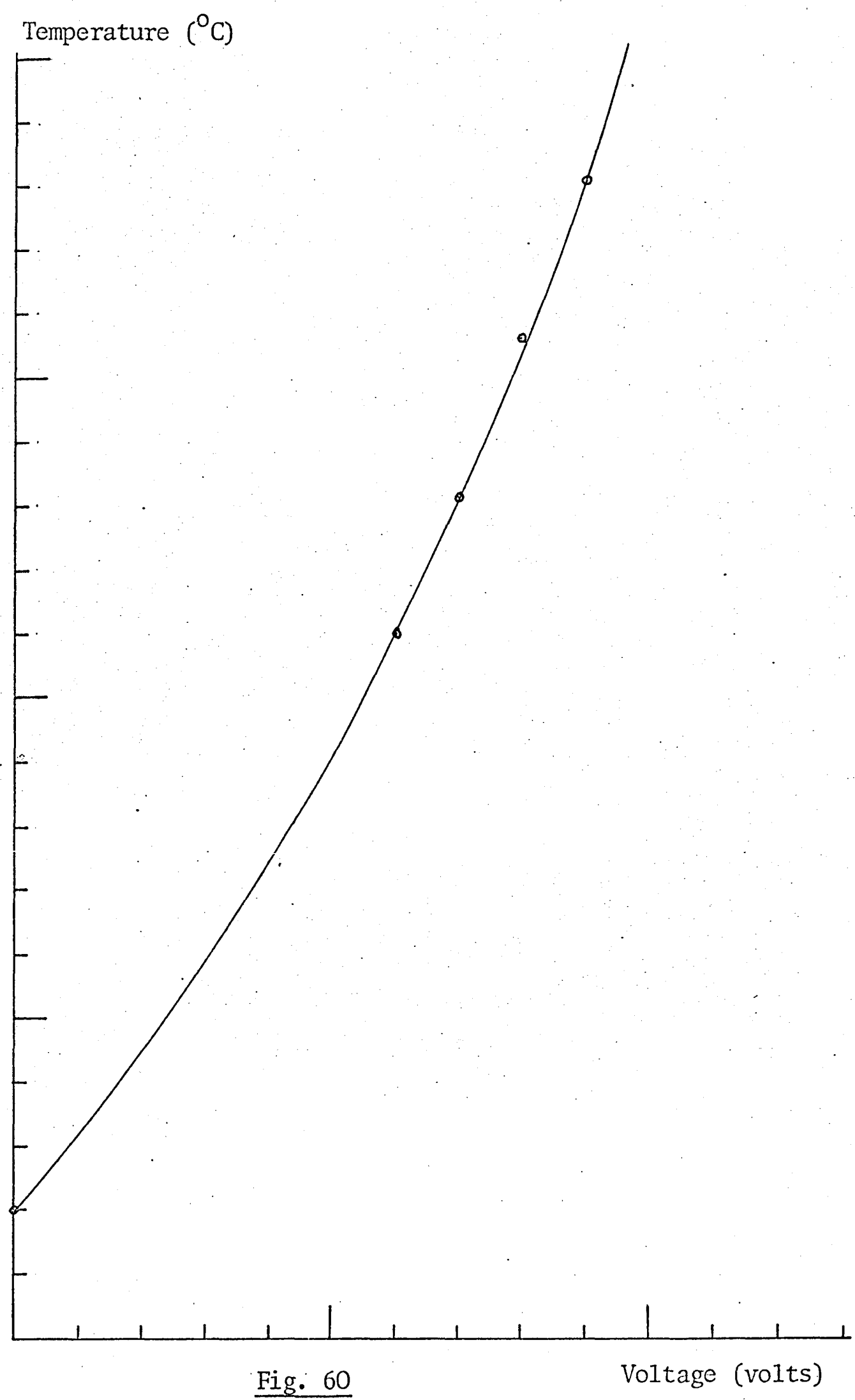

Temperature curve of the Raman heating cell 


\section{Light tight box}

A light tight box was designed for the sample compartment to provide air cooling and the sample tube can be put in sideways. There is also a small hole cut at the back of the box closed by a screwed plug which can be opened to observe the laser beam passing through the tube.

The cold air can get into the box by passing through the light tight tube under the sample compartment. The hot air will blow out through the baffled chimney at the top. The baffled chimney was designed so that it is light tight and can be screwed out when any observation of the laser beam that passes through the sample is required.

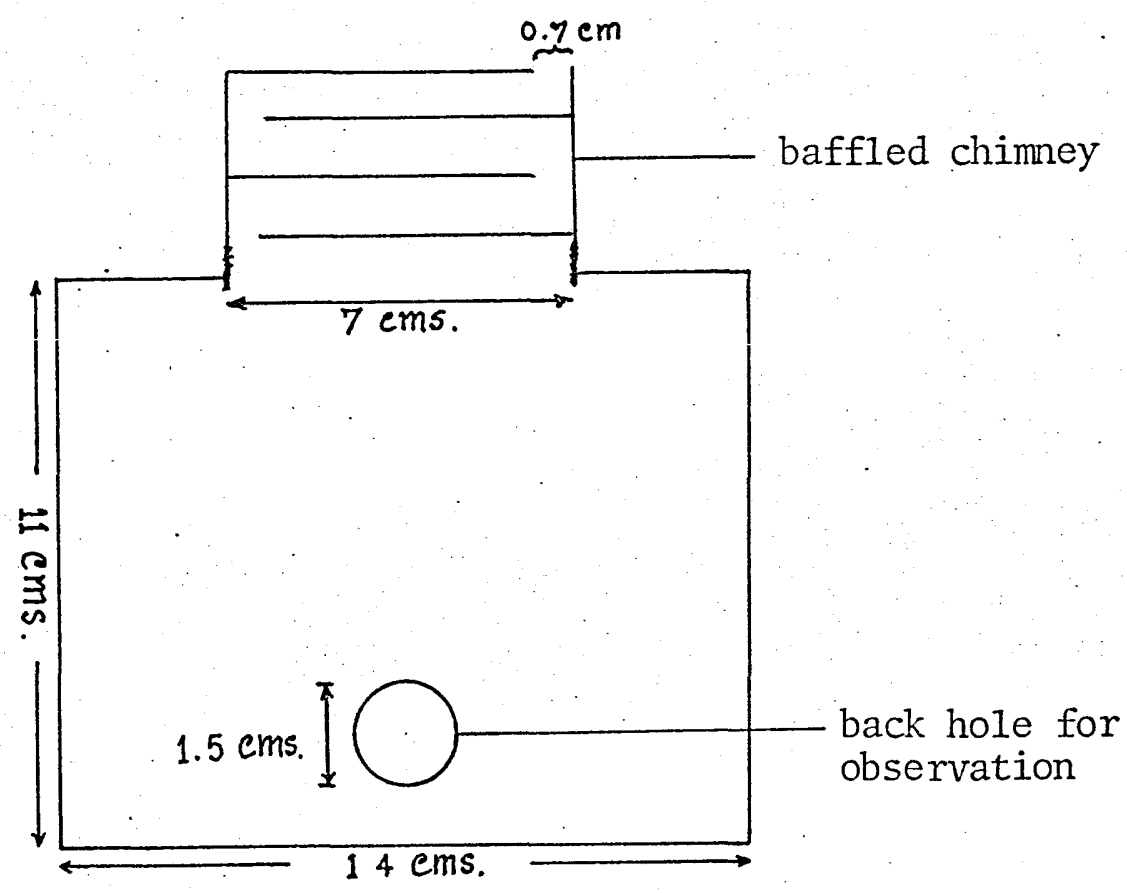

Fig. 61

Back view of the light tight box 


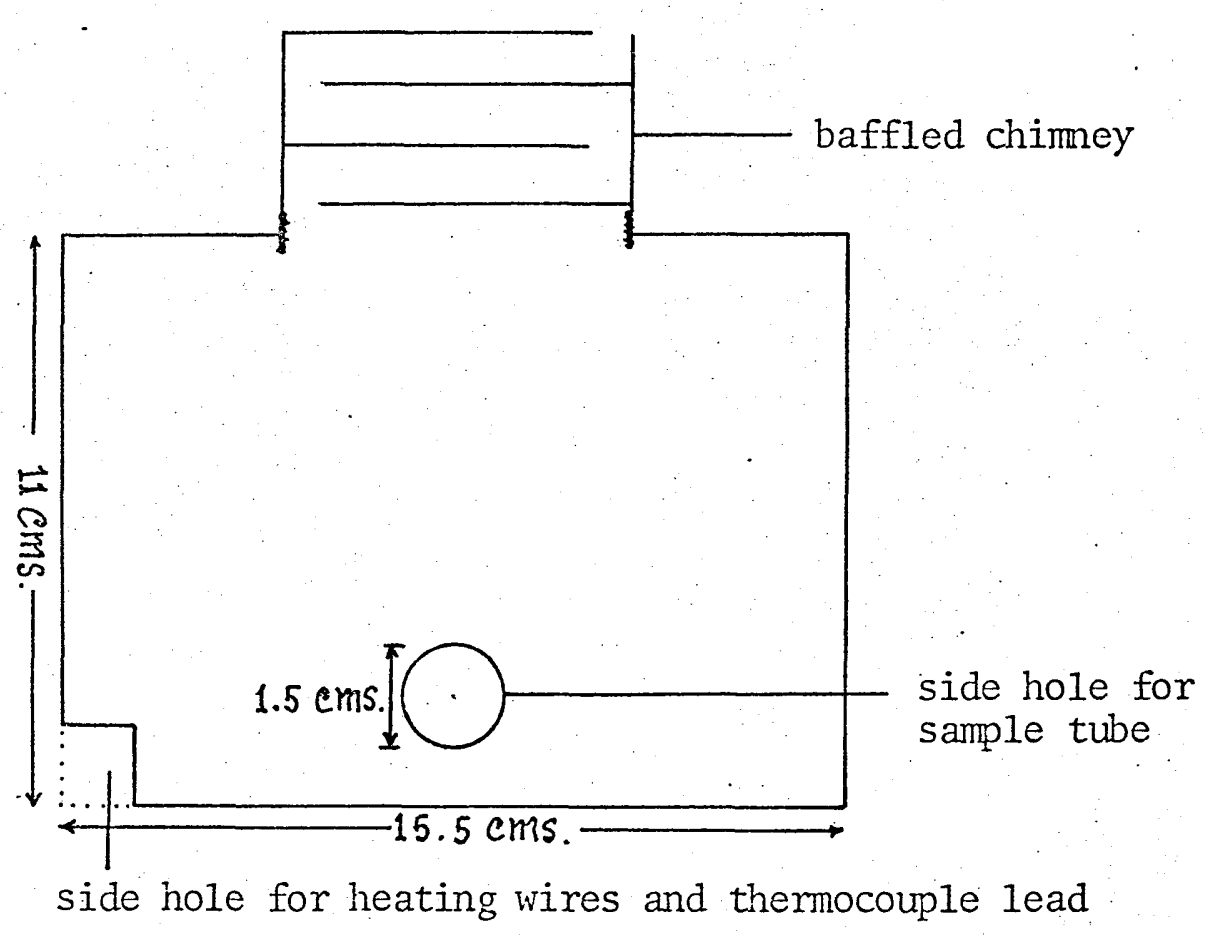

Fig. 62

Side view of the light tight box

The light tight box was made of brass and was painted matt black.

The inside of the aluminium tube of the oven was black anodised in order to reduce the amount of scattered exciting light which is reflected inside and some of which would enter the monochromator.

The first oven made was not anodised and showed a higher background than the anodised one when an empty sample cell was used. 


\subsection{Raman experiment}

At first the Spectan-pitysics to - We laser model 125 was used. Before any experinont was performed the instrument performance was chooked and found that

(a) the the - Ne laser power had gone down to 58 mo (used to provide 74 mi);

(b) the efficiency of the detector has gone dom nearly 10 tines. This was detected by compring the spectrm of $\mathrm{CCl}_{4}$ in a capillary twe with sinilar conditions,

(c) at high sensitivity the detector is very sensitive to the stray light which comos from light leskage at the edges of the double monochromator box. This problen was elininated by covering the whole nonochronator with black polyethylene.

The instrument was lined up by using a $\mathrm{CCl}_{4}$ vapour tube. At the beginning a sample tube was prepared using a pyrex ampoule of $1.2 \mathrm{~cm}$ dianster. This tube, containing anhydrous oxalic acid, was heated up sloviy and evacuated at the sane tims. The terperature was kept at $120^{\circ} \mathrm{C}$ for a while then the tube was seaied off and used as sample tubo.

The experiment was performed by heeting the striple up and necording the spectra at different temperatures from $60^{\circ} \mathrm{C}$ to $130^{\circ} \mathrm{C}$ with high sensitivity. So far the spectra showed no band of gaseous oxalic acid at ail. 
The experiment was finally performed by heating the cell up to $145^{\circ} \mathrm{C}$. This time the tube exploded and caused damage to the collection lens. This suggested that at $145^{\circ} \mathrm{C}$ the decomposition products increased so fast and resulted in increasing of the total pressure inside the tube.

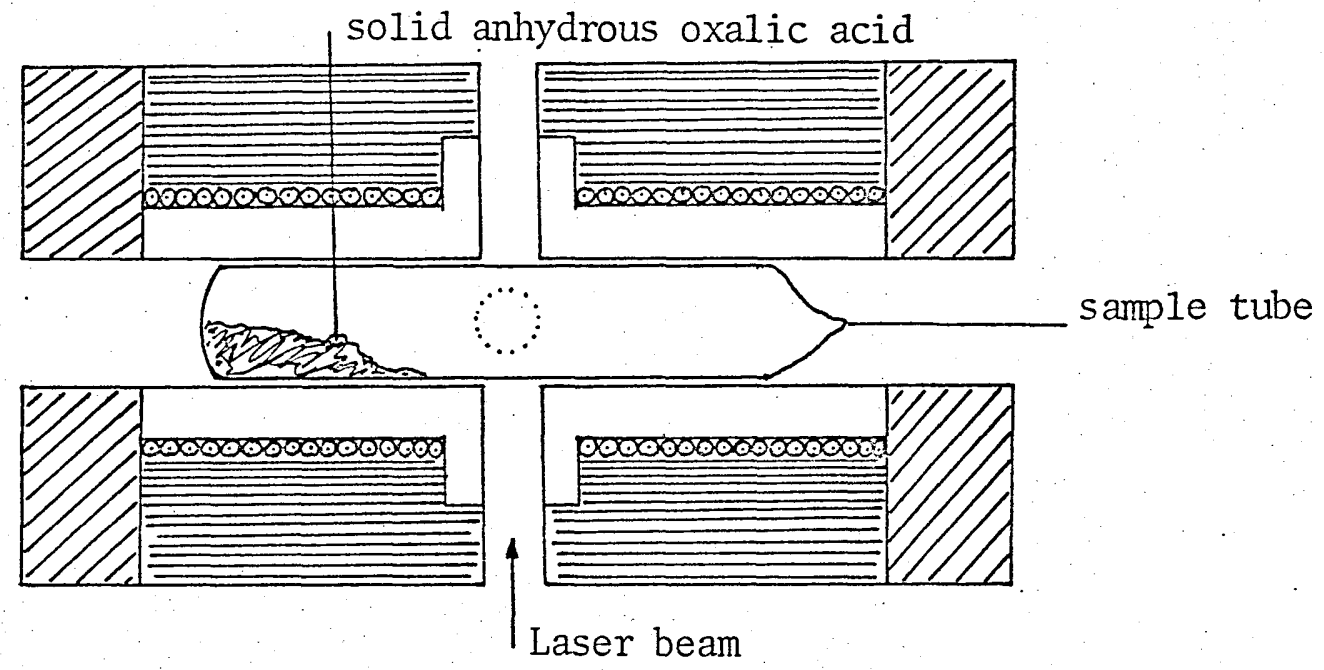

Fig. 63

$\mathrm{X}$-section of Raman heated cell and the sample tube

Handling the sample in this way there are several disadvantages:

(i) The decomposition products cause the increase of background level at high sensitivity.

(ii) At high temperature the total pressure increases fast and can cause an explosion.

(iii) Because the gaseous oxalic acid bands are very weak, it is necessary to heat the tube to more than $145^{\circ} \mathrm{C}$, giving the above mentioned disadvantages.

The sample tube was modified by using a long pyrex glass tube, sealed at one end and the other end connected to the vacuum 
pump. This tube contained anhydrous oxalic acid at the closed end as shown in Fig. 64. The tube was heated up and evacuated continuously. Although we heated up to $160^{\circ} \mathrm{C}$, the recorded spectra showed no bands of gaseous oxalic acid.

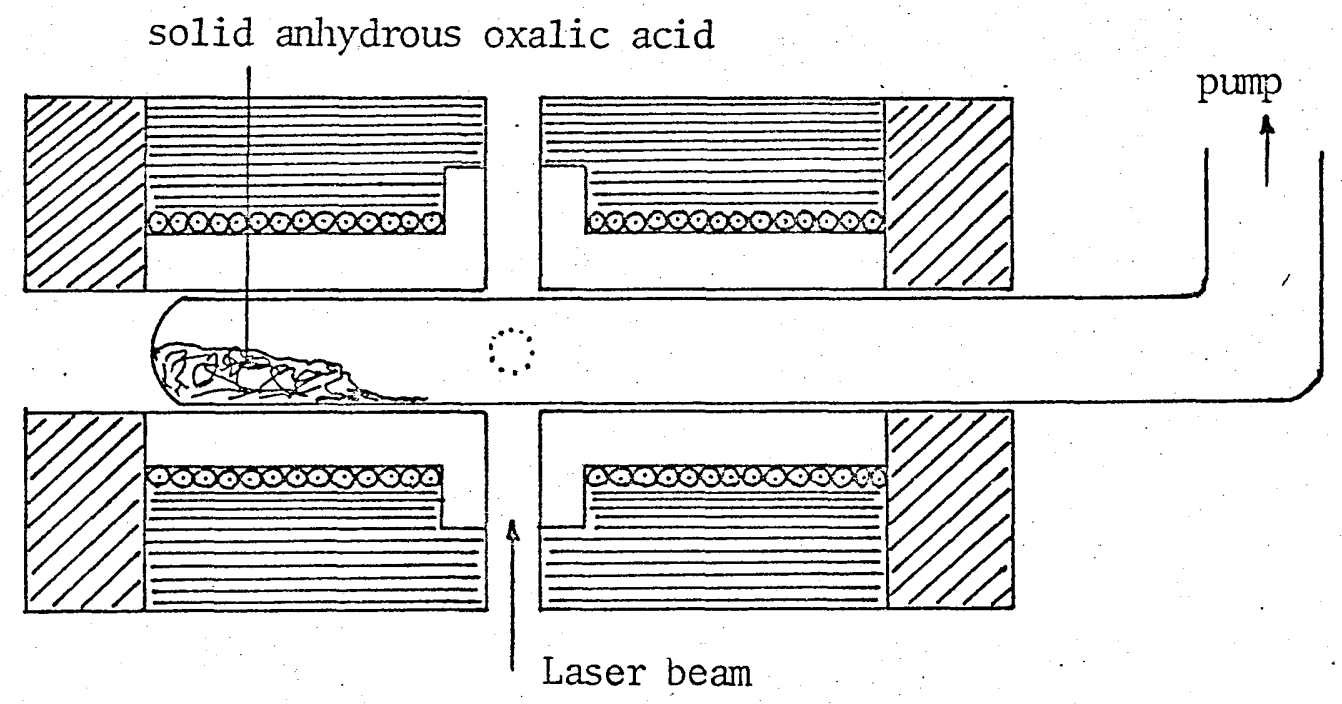

Fig. 64

X-section of the heated cell and the modified sample tube

The experiment was performed again by heating the tube up to $160^{\circ} \mathrm{C}$. This time the sample tube was evacuated before heating and then the vacuum tap was closed. In this way some decomposition products, which emerged from the hot end, condensed on the cooler end and resulted in reducing the pressure in the sample tube. Again the spectra recorded still showed no bands of gaseous oxalic acid.

From the above experiments it is obvious that the gaseous oxalic acid bands are very difficult to detect under these circumstances. This suggested that for further experiments the following 
itens are required:

(i) A much more powerful lasor.

(ii) A becter photomultiplier detector if possible.

(iii) It may be necessary to heat the sample over $160^{\circ} \mathrm{C}$ in order to increase the gaseous oxalic acid vapour pressure.

The experiment was perforned again with a Spectra-Physics 164 Argon ion laser. The power of the $4880 \AA$ ine is 1.7 watts and the power of the $5145 \AA$ line is 2.2 watts. With this powerful argon laser it has the advantage that the instrument can be peaked up using an $I_{2}$ vapour tube as the resonance flworescence lines of $I_{2}$ vapour are very strong. Before starting the experiment several trials were made and it was found that

(i) with the filter the transmittance of the bean at the sample position is 268 and without filter the laser beam at the sample position is 689 of the total intensity. This suggested that it is mach better to take the spectrum without the filter as we need maximm laser intensity for weak bands;

(ii) by placing a concave mirror on the top of the heating cell to reflect the transmitted bean back through the sample it was found thet the intensity of the band increased nearly twice;

(iii) although the $4880 \AA$ line has some advantages over the $5145 \AA$ line (as the intensity of the band varies with the fourth power of the frequencies), it gives more non-lasing lines. These non-lasing lines may 
coincide with the gaseous oxalic acid bands and make them undetectable. With $5145 \AA$ 政e no non-lasing lines could be detected between the region of $150 \mathrm{~cm}^{-1}$ to $2000 \mathrm{~cm}^{-1}$. So it was much more convenient to use the $5145 \stackrel{\circ}{\mathrm{A}}$ line without the filter.

This time anhydrous oxalic acid was filled in a silica tube of similar shape as shown in Fig. 64. The tube was evacuated and sealed. The sample was heated up to a maximun temperature of $180^{\circ} \mathrm{C}$. Several spectra of gaseous oxalic acid were recorded at different equilibrium temperatures. This time several bands of gaseous oxalic acid were observed (see 20.4) .

At temperatures above $185^{\circ} \mathrm{C}$ the rate of decomposition is much faster and the intensities of these bands decreased.

20.4 Raman results and the assignment

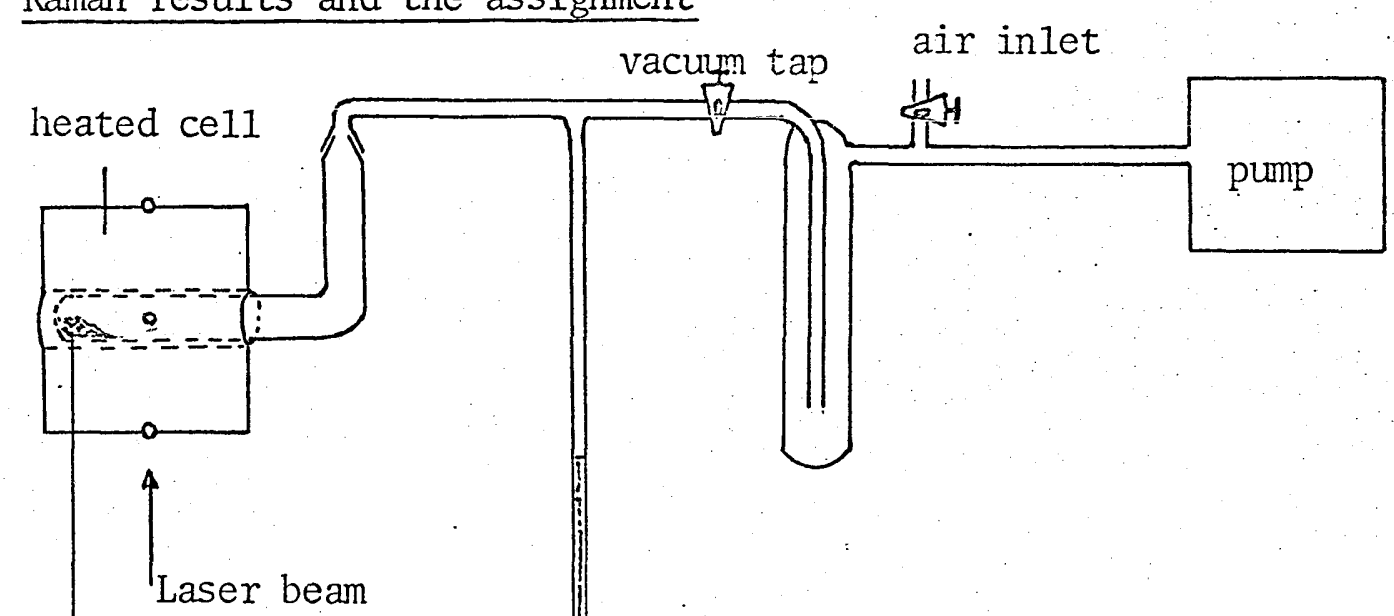

Manometer

soli.d anhydrous oxalic acid 
The instrument was lined us using an $I_{2}$ vapour tube. A silica tube of $1.2 \mathrm{~cm}$ dianeter containing anhydrous oxalic actd was placed in the heated cell and evacuated. The vacuren valve was closed before heating the cell up. Fig. 65 shows the heated cell was connected to a momotor and e pump during the experiment.

Spectra of gaseous oxalic acid were taken under the following settings :

$$
\begin{aligned}
\text { laser power } & =2 \text { watts } \\
\text { detector H.T. } & =2 \text { kilovolts } \\
\text { entrance slit } & =0.5 \text { millimeter } \\
\text { midale slit } & =0.5 \text { nillimoter } \\
\text { exit slit } & =0.5 \text { millimeter }
\end{aligned}
$$

plus conditions given in Table 21. The vapour pressure of oxalic acid is givan in section 20.2 .

Figures 66 to 77 show spectra of gaseous oxalic acid taken at different temperatures. It was noticed that the bands at $267 \mathrm{~cm}^{-1}, 610 \mathrm{~cm}^{-1}$ and a broad band between $300 \mathrm{~cm}^{-1}$ to $500 \mathrm{~cm}^{-1}$ vere recorded even in the spectrum of the cold empty cell. These bands were assigned to the scattering caused by the silica tube.

Figures 66 and 67 show spectra recorded from $200 \mathrm{~cm}^{-1}$ to $2300 \mathrm{~cm}^{-1}$ in which anhydrous oxalic acid was heated up to approximately $126^{\circ} \mathrm{C}$. At this temperature no bands of geseous oxalic acid were observed except that of $\mathrm{N}_{2}, \mathrm{O}_{2}, \mathrm{CO}_{2}$, and the silica tube. 


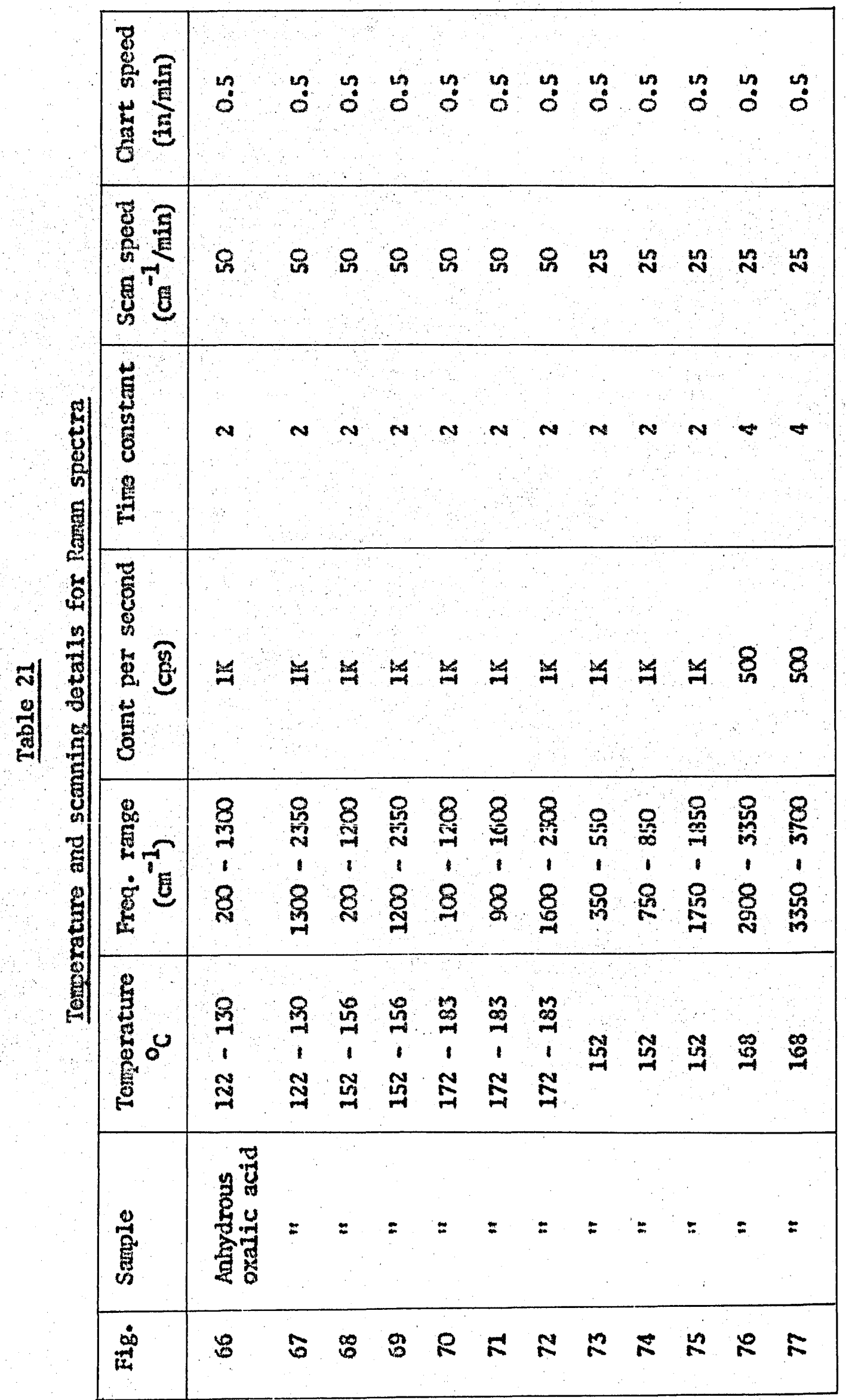




\begin{tabular}{|c|c|c|c|c|}
\hline 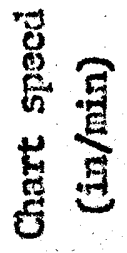 & in & $\stackrel{13}{\sim}$ & n & ? \\
\hline 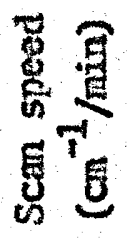 & 尽 & 呙 & 员 & 용 \\
\hline 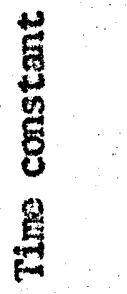 & 8 & $\dot{0}$ & 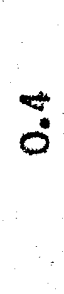 & $N$ \\
\hline 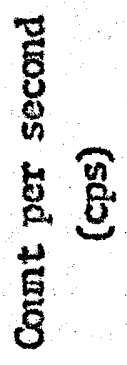 & 诜 & 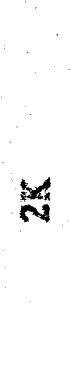 & 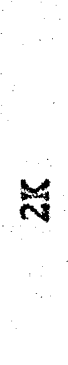 & $\approx$ \\
\hline 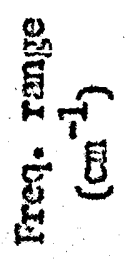 & $\begin{array}{l}8 \\
2 \\
1 \\
8 \\
0\end{array}$ & 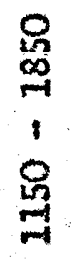 & $\begin{array}{l}8 \\
\text { n } \\
1 \\
8 \\
\text { ก }\end{array}$ & $\begin{array}{l}\frac{8}{4} \\
1 \\
8 \\
8\end{array}$ \\
\hline 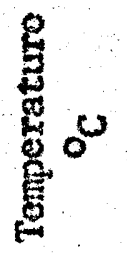 & 焉 & I & 9 & $\underset{7}{9}$ \\
\hline$\frac{9}{8}$ & 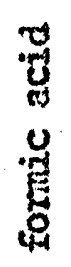 & $=$ & $=$ & ${ }_{i v}^{0}$ \\
\hline 悹 & $\stackrel{2}{\sim}$ & $\stackrel{R}{n}$ & 8 & $\infty$ \\
\hline
\end{tabular}


Figures 65 and 60 shor spectra of gaseous oxaltc acid recorded from $200 \mathrm{cal}^{-1}$ to $230 \mathrm{~cm}^{-1}$ at aproximatoly $154^{\circ} \mathrm{C}$. Figures 70 to 72 are the best spectra of gascous oxalic acid recorded so far from $200 \mathrm{~cm}^{-1}$ to $2500 \mathrm{~cm}^{-1}$ at approinately $177.5^{\circ} \mathrm{C}$.

Spectra were recorded up to $3700 \mathrm{~cm}^{-1}$. At high frequency three non lasing lines, two fomic acia bands and an $y_{2}$ o band were observed but none for gascous oxalic acid as shown in Fig. 76, (77).

Apart from the scattering caused by the sllica tube 18 bands were observed. Their essighent is given in Table 22.

for the purpose of correct assignment the spectra of formic acid, $\mathrm{CO}_{2}$ and $\mathrm{H}_{2} \mathrm{O}$ were twen at temporatures between $110^{\circ} \mathrm{C}-130^{\circ} \mathrm{C}$. A table of $\mathrm{CO}_{2}$ bands hith thoir assignant is givon in lerzberg (2 p.274). The swporting reforences are (13), (130).

Figures $78,79,80$ show spectra of formic acid between $500 \mathrm{~cm}^{-1}$ to $3700 \mathrm{~cm}^{-1}$ at aproxinately $120^{\circ} \mathrm{C}$. The spectrm of vater was recorded from $200 \mathrm{~cm}^{-1}$ to $3700 \mathrm{~cm}^{-1}$ at amproxinately $110^{\circ} \mathrm{C}$. Arart from several bands due to pure rotation at $10 \%$ froquency range there is only one vibrational band of uater observed in the Raman spectron at $3655 \mathrm{~cm}^{-1}$ as shom in Fis. 81 .

It was noted that $\mathrm{CO}_{2}, \mathrm{O}, \mathrm{O}_{2}, \mathrm{~A}_{2}$ bends are very sharp and ensy to idantisy. As $\mathrm{OO}_{2}$ and $\mathrm{CO}$ are decomposition products, their spactra can be used for mupeses of fremuency measurement. 
Table 22

Osserved Ramn bands and thoir assimmont

\begin{tabular}{|c|c|c|}
\hline Band position & $\begin{array}{l}\text { Spectrum } \\
\text { number }\end{array}$ & Assigment \\
\hline 267 & 66 & silica tube \\
\hline $300-500$ & 66 & siitca tube \\
\hline 405 & 70 & gaseous oxalic acid \\
\hline 610 & 66 & silica tube \\
\hline $\left.\begin{array}{l}800 \\
825\end{array}\right\}$ & 70 & gaseous oxalic acid \\
\hline 1195 & 70 & gascous oxalic acid \\
\hline 1267 & 71 & $\left(3 v_{2}-v_{2}\right) \quad C o_{2}$ \\
\hline 1286 & 72 & $2 v_{2}$ \\
\hline 1383 & 71 & $\omega_{2}$ \\
\hline 1409 & 71 & $\left(v_{1}+v_{2}-v_{2}\right) \infty_{2}$ \\
\hline 1423 & 71 & gaseous oxallc acid \\
\hline 1550 & 67 & $\mathrm{O}_{2}$ \\
\hline 1800 & 72 & gasaous oxalic acid \\
\hline 2143 & 69 & $\infty$ \\
\hline 2330 & 67 & $N_{2}$ \\
\hline 2954 & 76 & formic acid \\
\hline 3086 & 76 & non lasing line \\
\hline 3237 & 76 & non lasing line \\
\hline 3422 & 77 & noil lasing line \\
\hline .3573 & 77 & formic acid \\
\hline 3655 & 77 & $\mathrm{in}_{2} \mathrm{O}$ \\
\hline
\end{tabular}




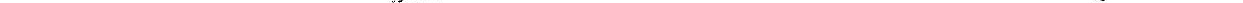



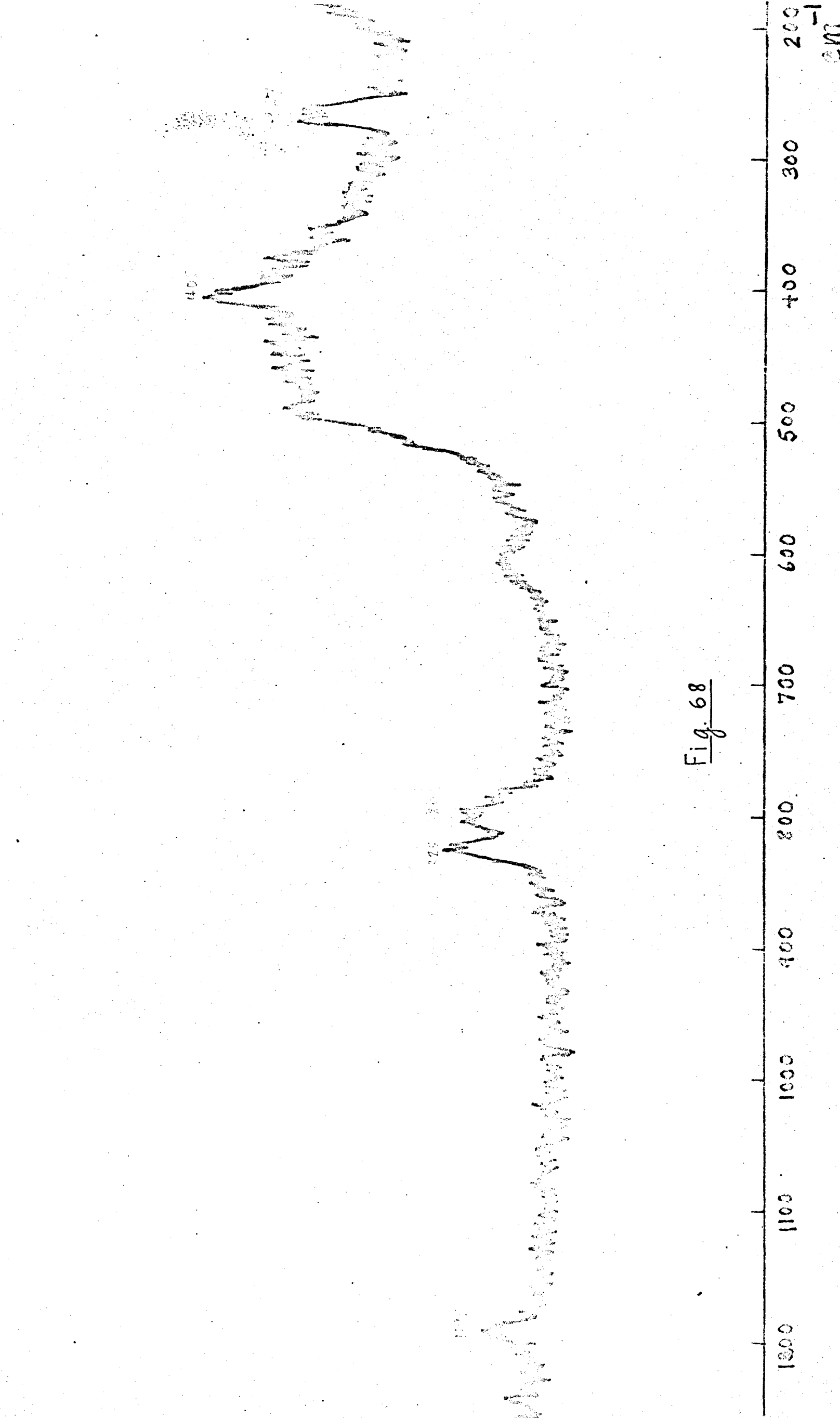

$-18$

$\stackrel{\circ}{8}$

0
0
+

8
0
6

0
3

$+8$

8

3

요

10 


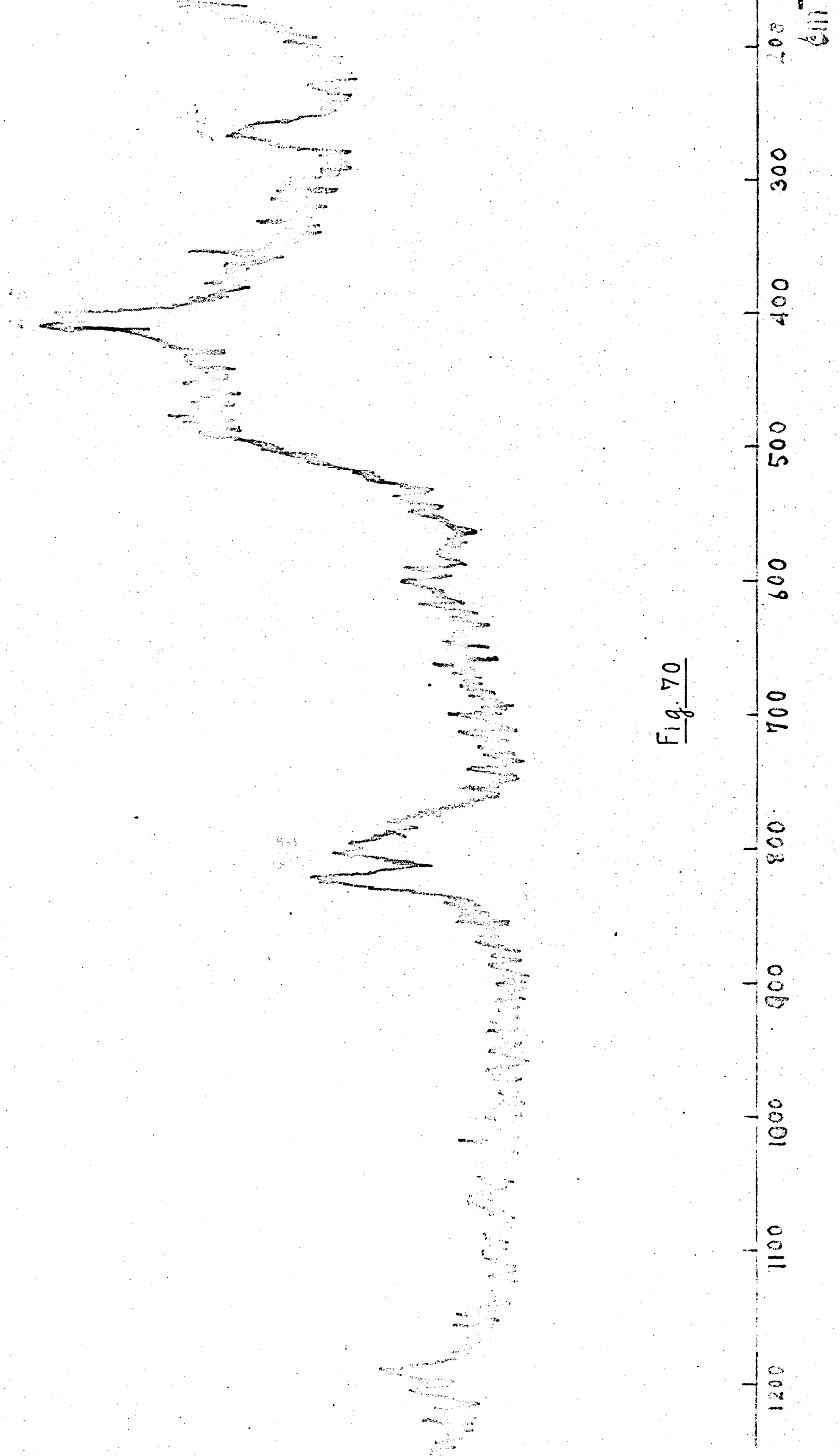


Fig. 71

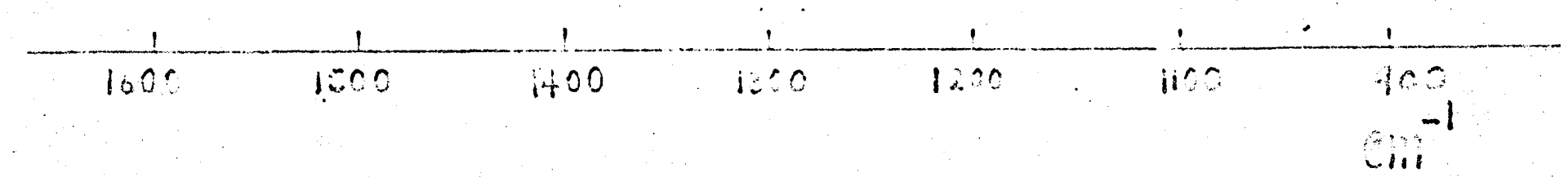



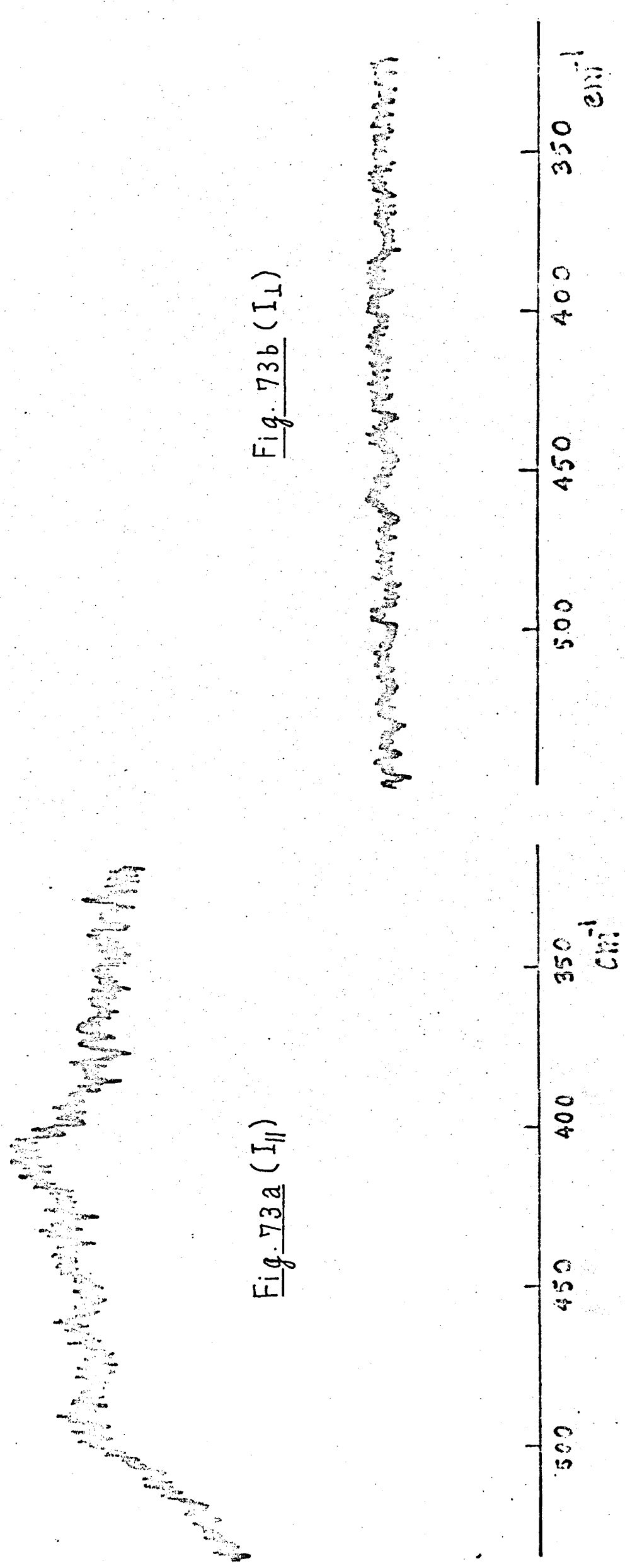

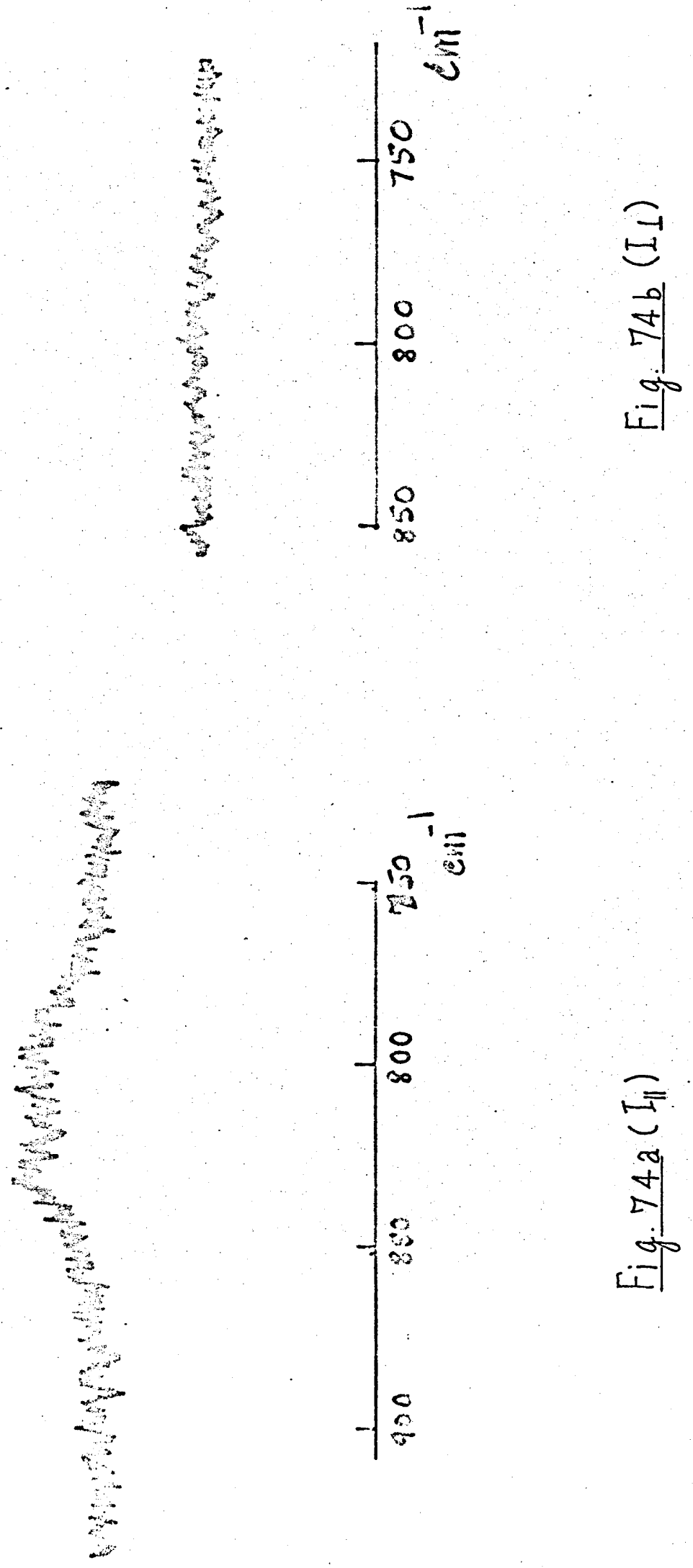

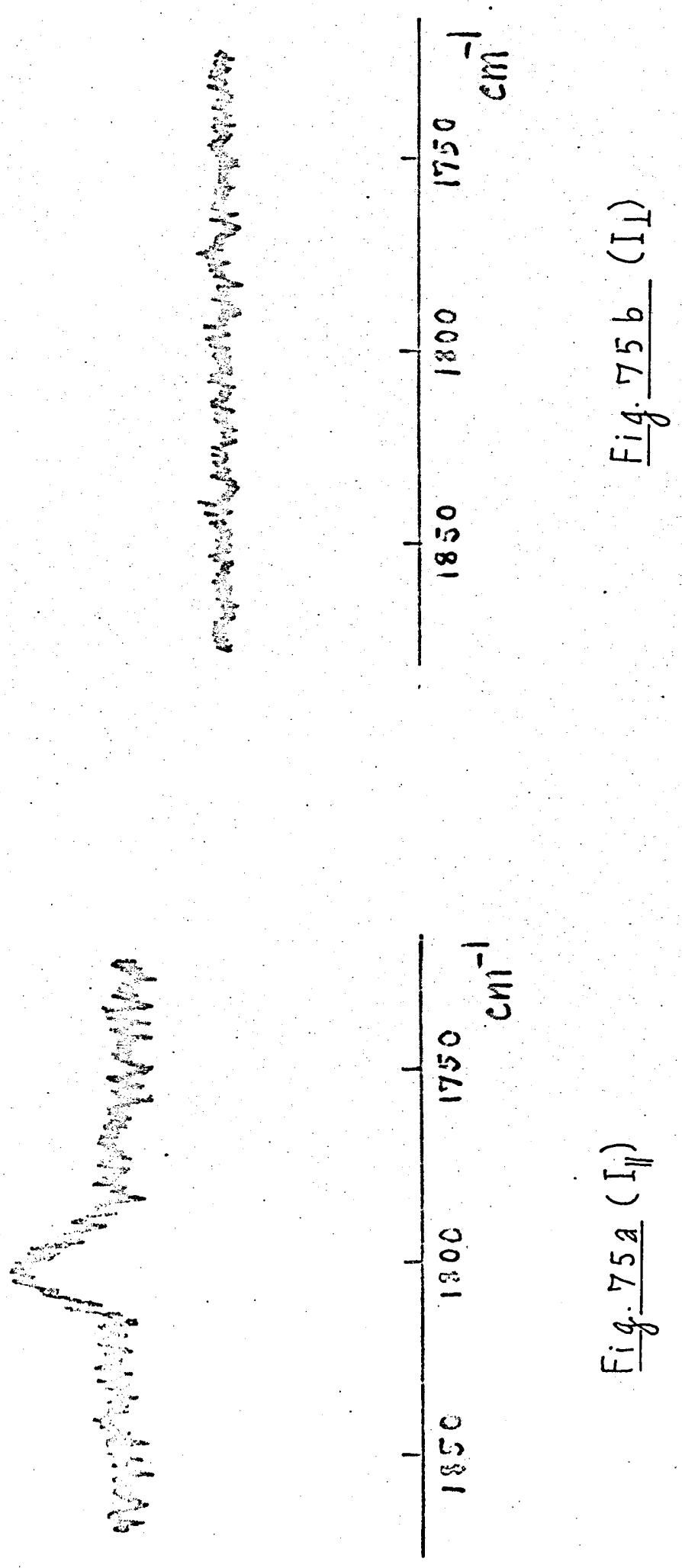


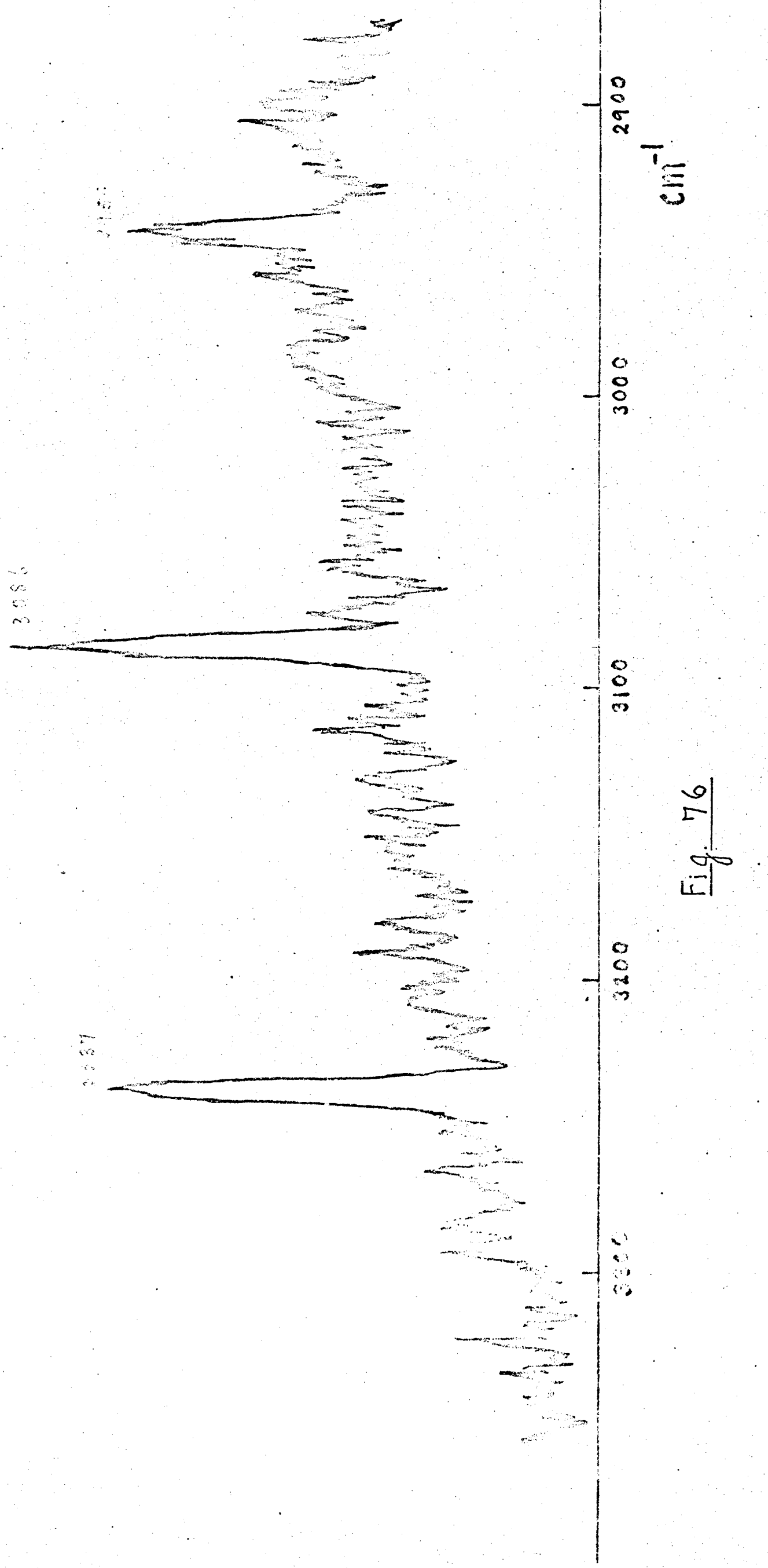




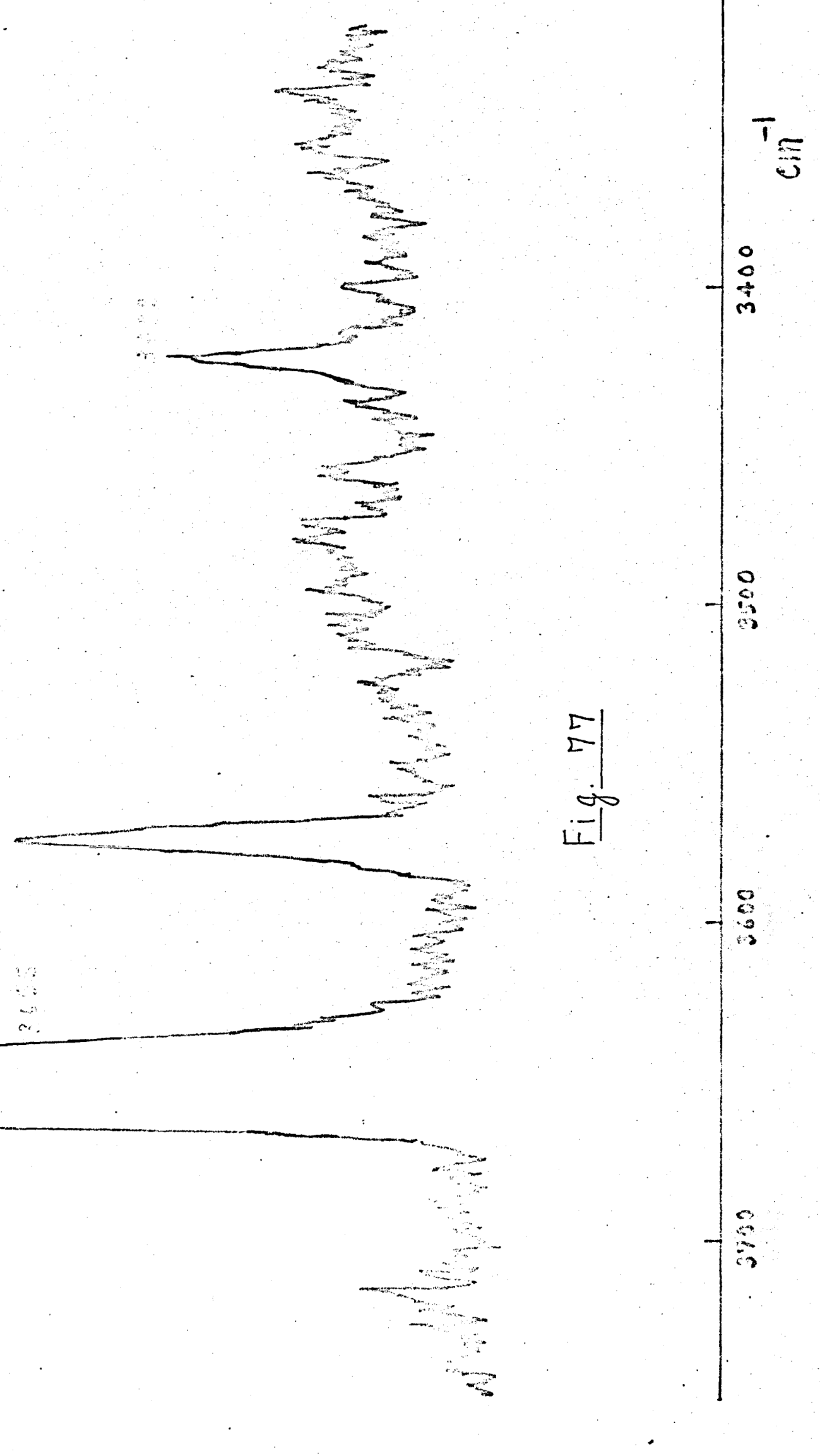




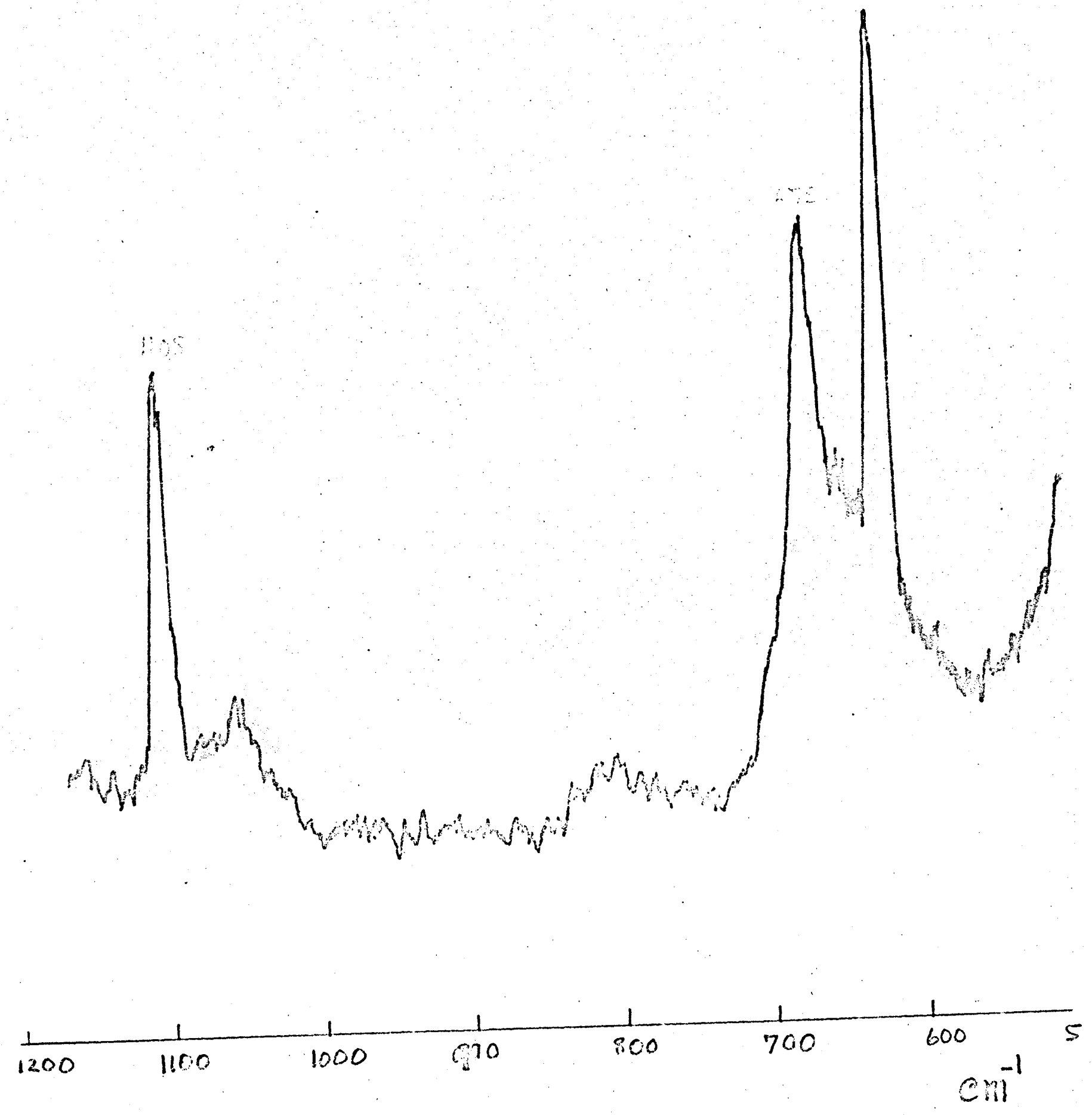

Fig 78 


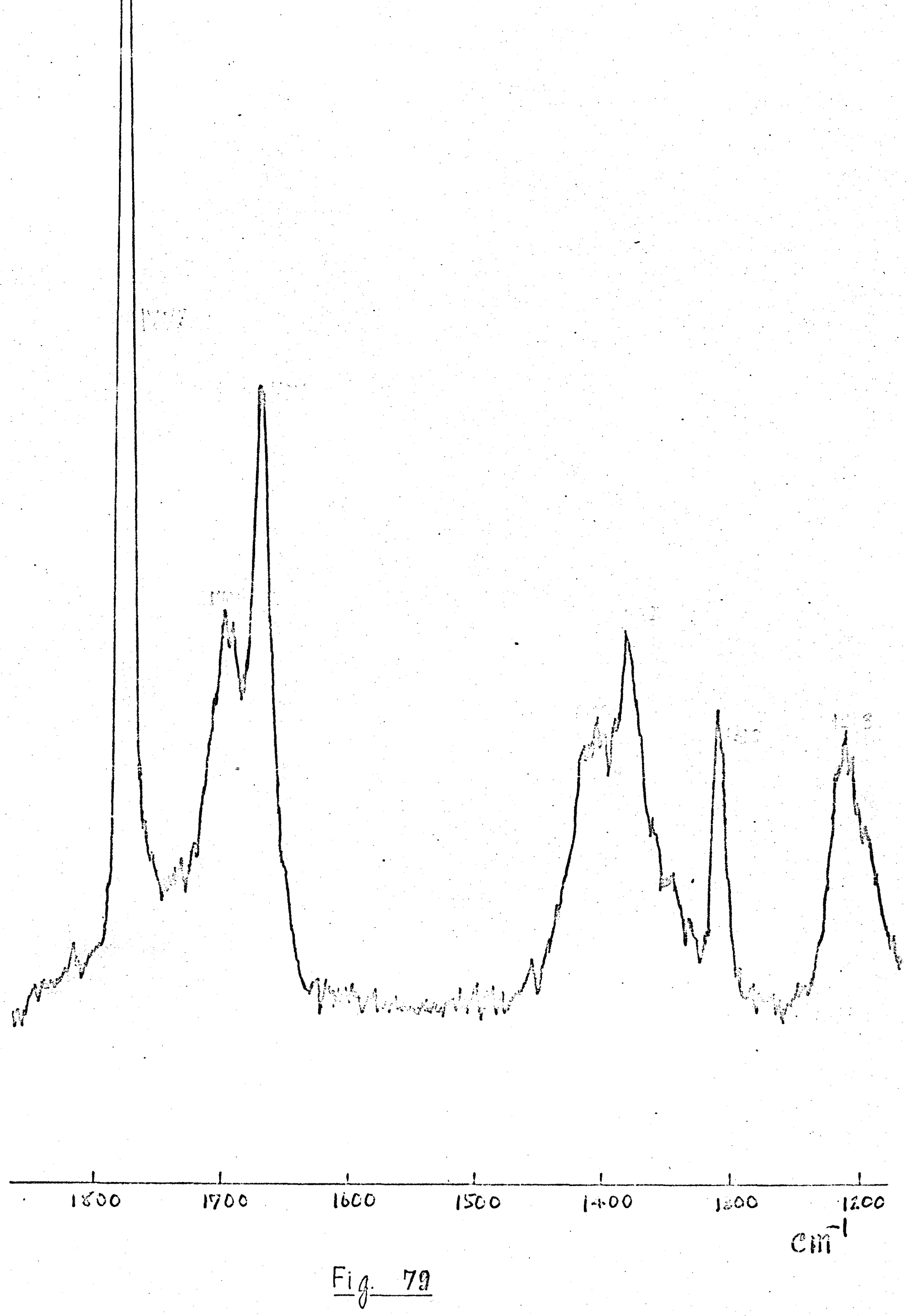




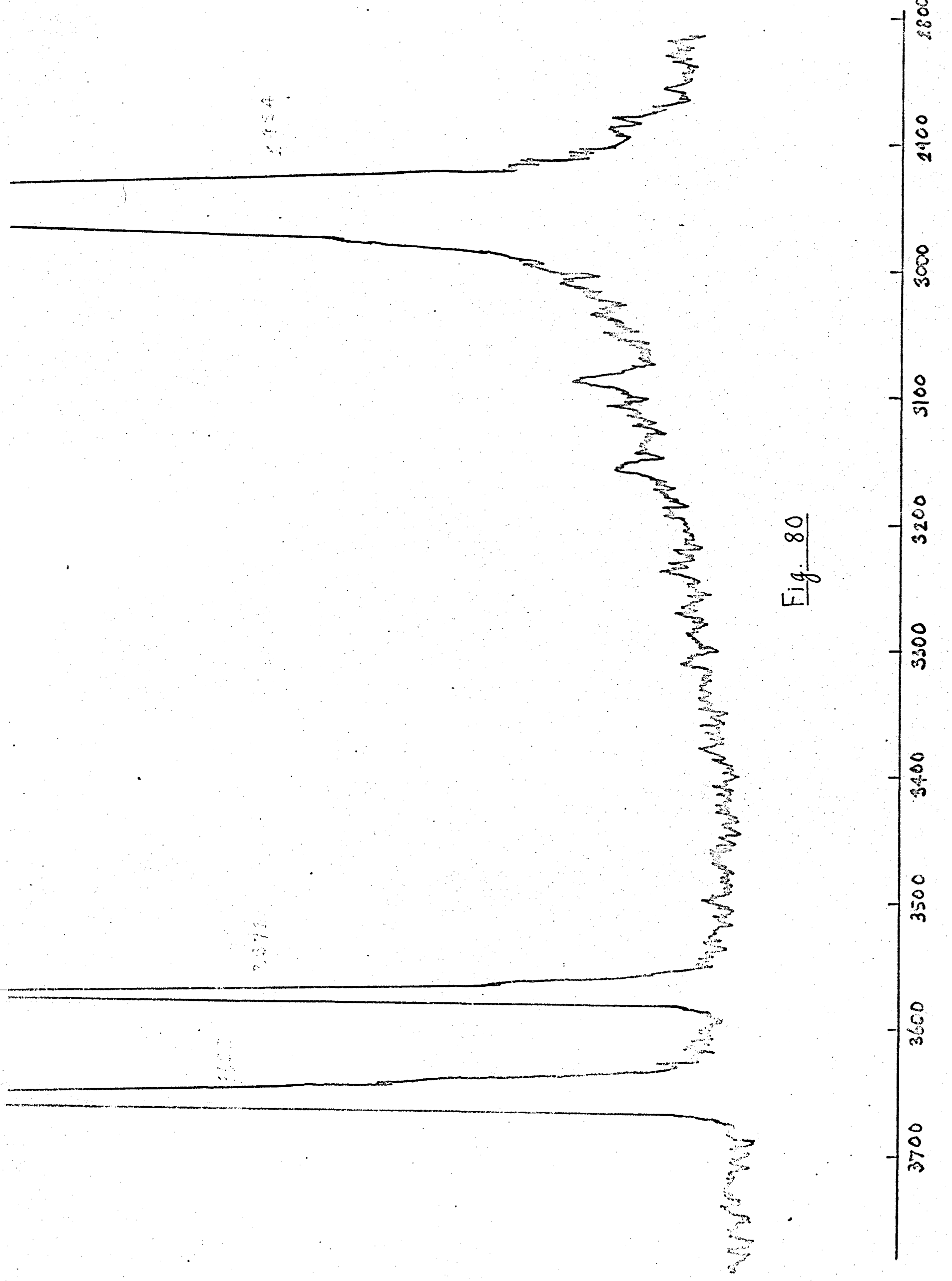



Assigmant of the gaseous oxalic acid bands in the Raman spectrum was easily made because

(1) Oxalic acid band shapes are very distinctive from those of $\mathrm{\infty}_{2}, \infty, \mathrm{O}_{2}, \mathrm{~N}_{2}$, and $\mathrm{H}_{2} \mathrm{O}$.

(2) Between $200 \mathrm{~cm}^{-1}$ to $1900 \mathrm{~cm}^{-1}$ no fomic acid bands and non lasing lines wore observed in the spectra of gaseous oxalic acid.

(3) Gaseous oxalic acid bands at $405 \mathrm{~cm}^{-1}, 812 \mathrm{~cm}^{-1}$ and $1800 \mathrm{~cm}^{-1}$ are strong enough for depolarization measurements. As shown in figures $73,74,75$ these bands are completely polarized. Thus, they were assigned to $\mathrm{Ag}$ inplane fundanontals, symetric $\left(-\mathrm{C}_{5}\right)$ inplane angle bencing, symetric $\mathrm{C}-\mathrm{C}$ stretch, and symetric $\mathrm{COO}$ stretch respectively.

Although the band at $1195 \mathrm{~cm}^{-1}$ is weak and thus a depolarization moasurement cannot bo made, it was assigned to inplane fundamental $\mathrm{Ag}, \mathrm{C}-\mathrm{O}-\mathrm{H}$ symatric anglo bending, $v_{7}$, because no out of piane fundanentals of gaseous oxalic acid are expected at this frequency. The band at $1423 \mathrm{~cm}^{-1}$ is very weak and the existence is doubtful because the peak height is not much above the noise level. As shown in fig. 71 , this band sits at the beso of $1410 \mathrm{~cm}^{-1}$ line of $\mathrm{C}_{2}$. It was assigned to the fundamentel inplane symetric c-0 stretch, $v_{3}$, of gasoous oxalic acid for the following reasons:-

(1) According to my calculations there is a calculated Ag fundamental at approximately this position.

(2) It could probabiy be that this band corrosponds to the $1450 \mathrm{~cm}^{-1}$ band which was reported in the spectrum of solution and solid oxalic acid (51), (124). 
(3) It is reasonable to expect a $\mathrm{C}-0$ symetric stretching band to occur at this frequency.

20.5 Comparison between infrared and Raman experiments

Infrared and Raman experiments of the gaseous oxallc acid face different problems as follows:-

(1) In Ranan there is no interference caused by solid oxalic acid whereas in infrared a condensation of solid oxalic acia on the $\mathrm{KBr}$ windows is one of the main problems. (2) In infrared the heated cell was purmed continuously to eliminate interforence caused by decomposition products. In Raman it was found that:

(a) No gaseous oxalic acid bands were observed if the cell was punped continuously.

(b) Ho obvious interference was caused by the docomposition products. Wain products such as $\mathrm{CO}_{2}$, $\infty$, and $\mathrm{H}_{2} \mathrm{O}$ gave sharp and strong bands which are useful for frequency measurenents.

(3) Raman bands begin to appear at about $140^{\circ} \mathrm{e}$ whereas infrared absorption bends begin to appear at approxinately $65^{\circ} \mathrm{C}$.

(4) Raman band shanes are very distinctive from the others and thus they are onsily identified.

(5) Raman bands are much more difficult to detect than the infrered absorption bands and thus the Raman experinent needs

(a) a very accurate lining up;

(b) a powerful laser;

(c) a higher tomperature heated cell. 


\section{CAPTER 21}

FAR-INFRARED EXIERIMETT

It was intended to carry out infrared absorption measurethents in the far-infrared using an R.I.I.C. Fourier Transform Spectrometer 520. An R.I.I.C. 1 m gas cell wes rodified as shown in Iig. 82. The polythene windows were replaced by TPX (joly 4 nothyl pontene 1) which can be used up to $140^{\circ} \mathrm{C}$ or higher. The cell was wound with electric heacing tape in such a way that the window temperature was about $5-10^{\circ} \mathrm{C}$ higher than the nidale of the cell. This was checked before the cell was fitted to the interferoweter. Two $1^{\text {th }}$ thick pieces of Syidanio were used to insulate the cell from the juterferomoter housing and the detector unit. These pieces of Syndanio were grooved to take the Viton 0-rings and drilled to take the bolts. They were vacuun impregated (comercially) with Hidland Silicone M.S. 2728 in order to make then vacuun tight. The piece between the cell was the interferometer was satisfactory but the piece botween the cell and the detector was not. The detector compartment which was in connection with this plece could not be punped down to a low enough prossure.

The attompt to obtain the far infrared spoctrum was abandoned as there was not time to obtain more Syndenio and carry out the nachining and ingregnacion. 
CANPTER 22

CALCULATION AND TIE ASSIGAMENT OF THE OBSERVED BANDS

OE GASEOUS OXALIC ACID FREOUEVCIES

\subsection{The inplane force constants and the calculated frequencies of} gaseous oxalic acid

Calculations for the gaseous oxallc acid molecule were made using the following data:-

(1) Molecular paraneters given by Nahlovska, Nahlovsicy and Strand (73) were used. Due to the possibility of different positions of 11 atons in this molecule, the calculations were thade using both models suggested by then (seo section 13.3).

(2) In this present work the force constants for the inplane vibrations of gaseous oxalic acid were taken fron the values for formic acid and acetic acid monomers reported by Nakaroto and Kishida (39). Both sets of force constants were used to calculate the inilane vibrational frequencies for gaseous oxalic acid. The set of force constants taken from acetic acid nonomor gave a better fit, thus, this set of force constants was selected as an initial set of force constants for gaseous oxalic acid. Several force constant refinemonts wero nado. It was found that the least squares method could not be applied because of singularity of the ( $\mathrm{J}^{t} \mathrm{WJ}$ ) matrix. With the aid of the computer the refinements can be nade successfully by trial and error in which small changes of force constants can be judged from the 
Jacobian matrix and the discrepancy between the observed and calculated frequencies.

At the beginning measurements of the variance of the calculated and observed values were rade using only 8 observed frequencies, were observed in the infrared at $3475 \mathrm{~cm}^{-1}$, $1812 \mathrm{~cm}^{-1}, 1325 \mathrm{~cm}^{-1}$, and $1275 \mathrm{~cm}^{-1}$, the others were observed in the Raran at $1800 \mathrm{~cm}^{-1}, 1195 \mathrm{~cm}^{-1}, 812 \mathrm{~cm}^{-1}, 405 \mathrm{~cm}^{-1}$. For the following reasons only 8 were used in the beginning:-

(a) These bands cortainly exist.

(b) They bolong to oxalic acid.

(c) They are all inplane fundamentals.

It was found that the observed bands at $1423 \mathrm{~cm}^{-1}$ and $660 \mathrm{~cm}^{-1}$ are fitted well with the calculated frequencies for gaseous oxalic acia first mode1. The bands at $1423 \mathrm{~cm}^{-1}$ and $460 \mathrm{~cm}^{-1}$ are fitted well with the calculated values for the second node1. Thus, these bands were also included in measurements of the variance. That is, for both models the variance is measured fron 10 observed fundamentals.

\subsection{Calculation using first model}

$G$ and $P$ matrices for gaseous oxalic acid first nodel have been constructed in Chapter 15 and Chapter 16 respectively. With the aid of tire computer the calculations were porformed and the results are shown in tables belon.

Table 23 shows 4 sets of force constants, A, B, C, D, used to calculate the corresponding sets of calculated frequencies shown in Table 24. 
Table 23

Sets of force coistants

\begin{tabular}{|c|c|c|c|c|}
\hline Typo of force constants & $\begin{array}{l}\text { Set } A \\
\left(\mathrm{~m} / \mathrm{A}^{\circ}\right)\end{array}$ & $\begin{array}{l}\text { Set } B \\
\left(\mathrm{md} d / \mathrm{A}^{\circ}\right)\end{array}$ & $\begin{array}{l}\text { Set } C_{\text {. }} \\
\text { (nd } / A^{\circ} \text { ) }\end{array}$ & $\begin{array}{l}\text { Sot } D \\
\left.\text { (nind/ } / A^{\circ}\right)\end{array}$ \\
\hline $\mathrm{K}_{\mathrm{C}-\mathrm{C}}$ & 3.2 & 3.2 & 2.5 & 2.3 \\
\hline$\pi_{C=0}$ & 10.9 & 10.9 & 10.5 & 10.3 \\
\hline$E_{C-O}$ & 6.0 & 6.0 & 6.0 & 6.0 \\
\hline$x_{0-H}$ & 6.5 & 6.5 & 6.5 & 6.5 \\
\hline $\mathrm{H}_{\mathrm{C}-\mathrm{C} \infty \mathrm{O}}$ & 0.7 & 0.7 & 0.7 & 0.7 \\
\hline${ }^{H} \mathrm{C}-\mathrm{C}-\mathrm{O}$ & 0.2 & 0.2 & 0.2 & 0.2 \\
\hline $\mathrm{H}_{\mathrm{O}-\mathrm{C} \times \mathrm{O}}$ & 0.4 & 0.4 & 0.45 & 0.44 \\
\hline $\mathrm{H}_{\mathrm{C}-\mathrm{O}-\mathrm{H}}$ & 0.5 & 0.4 & 0.42 & 0.42 \\
\hline $\mathrm{F}_{\mathrm{C}_{1} \mathrm{O}_{3}}-\mathrm{F}_{\mathrm{C}_{2} \mathrm{O}_{6}}$ & 1.2 & 1.2 & 1.2 & 1.2 \\
\hline$F_{C_{1} O_{4}}=F_{C_{2} O_{7}}$ & 0.7 & 0.7 & 0.7 & 0.7 \\
\hline $\mathrm{F}_{\mathrm{O}_{3} \mathrm{O}_{4}}=\mathrm{F}_{\mathrm{O}_{6} \mathrm{O}_{7}}$ & 0.7 & 0.7 & 0.7 & 0.7 \\
\hline $\mathrm{F}_{\mathrm{O}_{4} \mathrm{O}_{6}}=\mathrm{F}_{\mathrm{O}_{3} \mathrm{O}_{7}}$ & 0.2 & 0.02 & 0.02 & 0.02 \\
\hline $\mathrm{F}_{\mathrm{C}_{2} \mathrm{H}_{5}}=\mathrm{F}_{\mathrm{C}_{1} \mathrm{H}_{8}}$ & 0.55 & 0.55 & 0.55 & 0.55 \\
\hline
\end{tabular}


Table 24

Sets of calculated frequencies

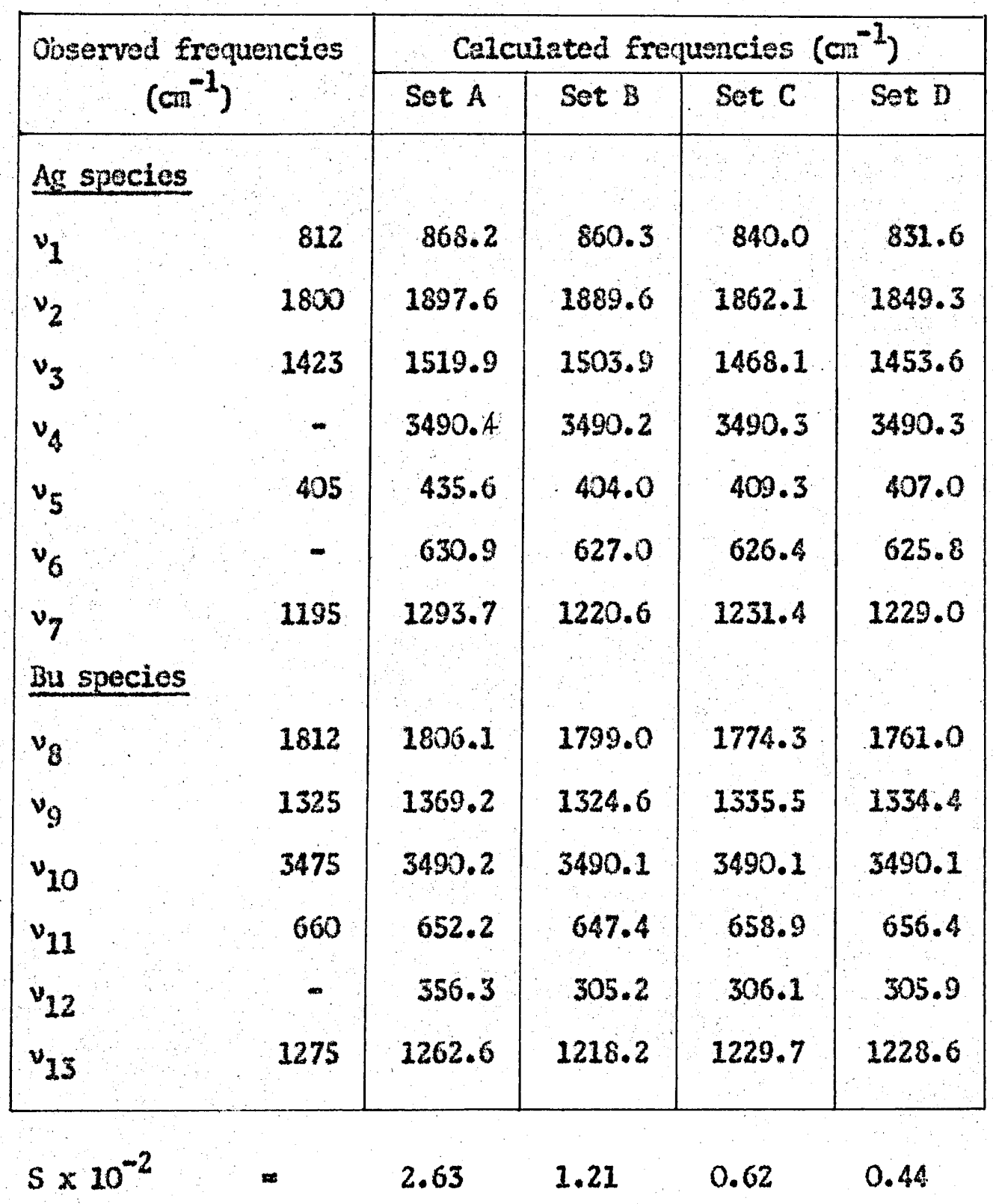


Measurenents of best fit are shown under Table 24. The variance $S$ is deftined as the sum of the squares of the percentage discrepancies between the calcuiated and the observod frequencies. Trat is

$$
s=\sum\left\{\frac{v_{\mathrm{cal} .}-v_{\text {obs. }}}{v_{\text {obs. }}} \times 100\right\}^{2}
$$

having a snaller variance set $D$ becomes a better set than the others. Thus, this set was used for the band assigmant and was also used to calculate tho potential energy distribution and the nodes of vibration.

The above refinements wore made by the trial and orror mothod in which small changes of force constants were adjusted with the help of the Jacobien natrix shown in Table 25. This matrix was constructed using the method described by Menn, Shinanouchi, Meal and Fano (24) (see section 7.3). 
22.3 Assignment of tho observed infrared and Raman bands, potential energy distribution and normal modes

Assignment of the observed infrared and Raman bands of gaseous oxalic acid to fit the calculated frequencies for the first model is shom in Table 26.

Table 20

\begin{tabular}{|c|c|c|c|c|c|}
\hline \multicolumn{2}{|c|}{$\begin{array}{l}\text { Observed frequencies } \\
\qquad\left(\mathrm{cm}^{-1}\right)\end{array}$} & \multicolumn{2}{|c|}{ Assigamenc } & \multirow{2}{*}{$\begin{array}{l}\text { Calculated } \\
\text { froquencies } \\
\qquad\left(\mathrm{cm}^{-1}\right)\end{array}$} & \multirow{2}{*}{$\Delta v=v_{\text {calc. }}-v_{\text {obs. }}$} \\
\hline Infrared & Raman & & & & \\
\hline 3560 & & $v_{2}+v_{8}$ & Bu & - & \\
\hline 3475 & & $v_{10}$ & $\mathrm{Bu}$ & 3490.1 & 15.1 \\
\hline 1812 & & $v_{8}$ & Bu & 1761.0 & -51.0 \\
\hline 1325 & & $v_{9}$ & Bu & 1334.4 & 9.4 \\
\hline 1275 & & $v_{13}$ & $\mathrm{Bu}$ & 1228.6 & -46.4 \\
\hline 660 & & $v_{11}$ & Bu & 656.4 & -3.6 \\
\hline 620 & & & $\mathrm{Au}$ & - & \\
\hline 460 & & & $\mathrm{Au}$ & - & \\
\hline & 1800 & $v_{2}$ & $\mathrm{Ag}$ & 1849.3 & 49.3 \\
\hline & 1423 & $v_{3}$ & $\mathrm{Ag}$ & 1453.6 & 30.6 \\
\hline & 1195 & $v_{7}$ & $\mathrm{Ag}$ & 1229.0 & 34.0 \\
\hline & 812 & $v_{1}$ & $\mathrm{As}$ & 831.6 & 19.6 \\
\hline & 405 & $v_{5}$ & $\mathrm{Ag}$ & 407 & 2.0 \\
\hline
\end{tabular}

Potential onorgy distribution

The potential energy distribution of different vibrations was calculatod using the mothod of Vorino and kuchitsu (30) and the results are in Tablo 27. 


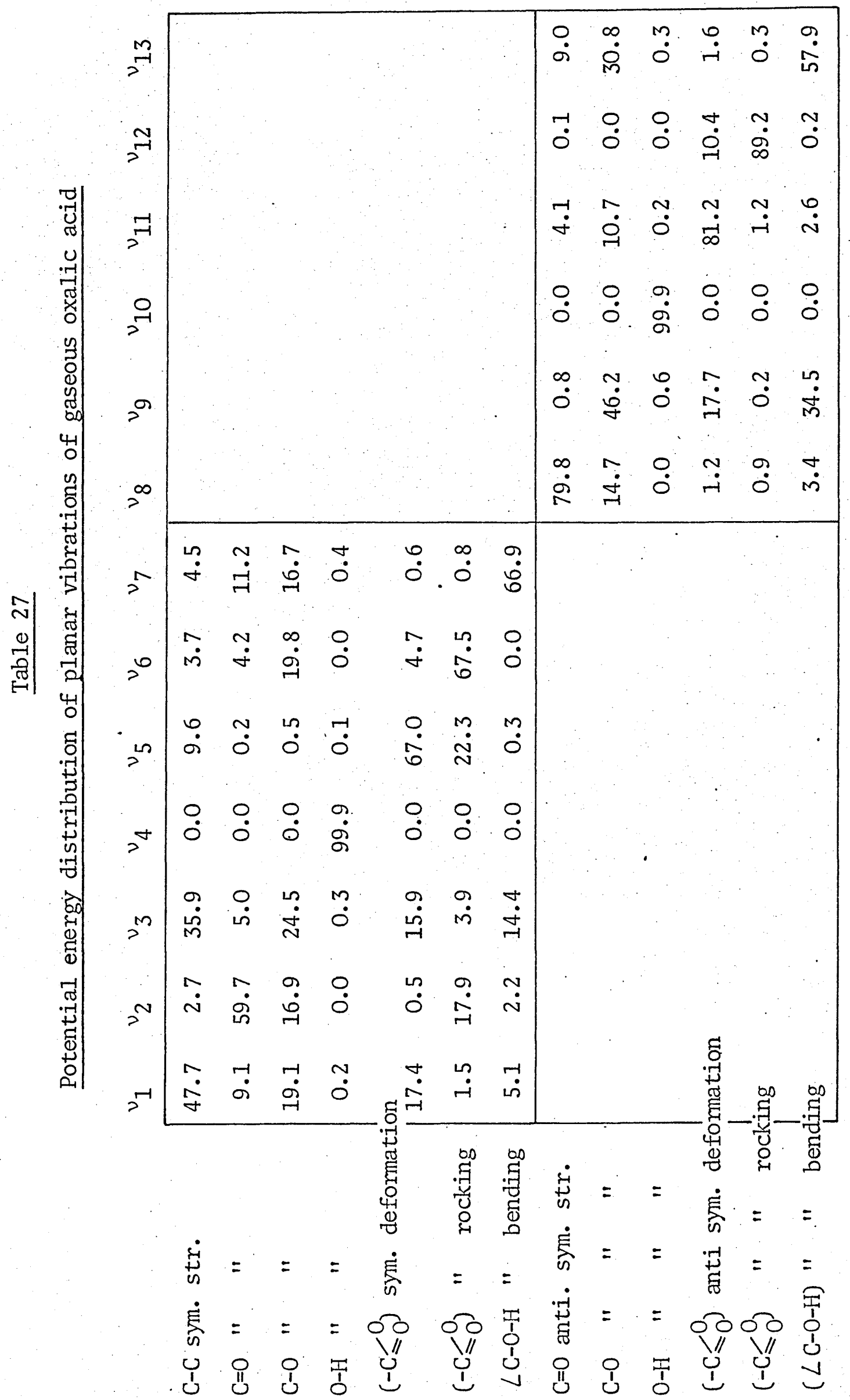


Modes of vibration

The $\&$ vectors in mass adjusted displacenent co-ordinates were calculated and the 13 inplane nodes of vibration were plotted by the computer as shom in the following figures. 


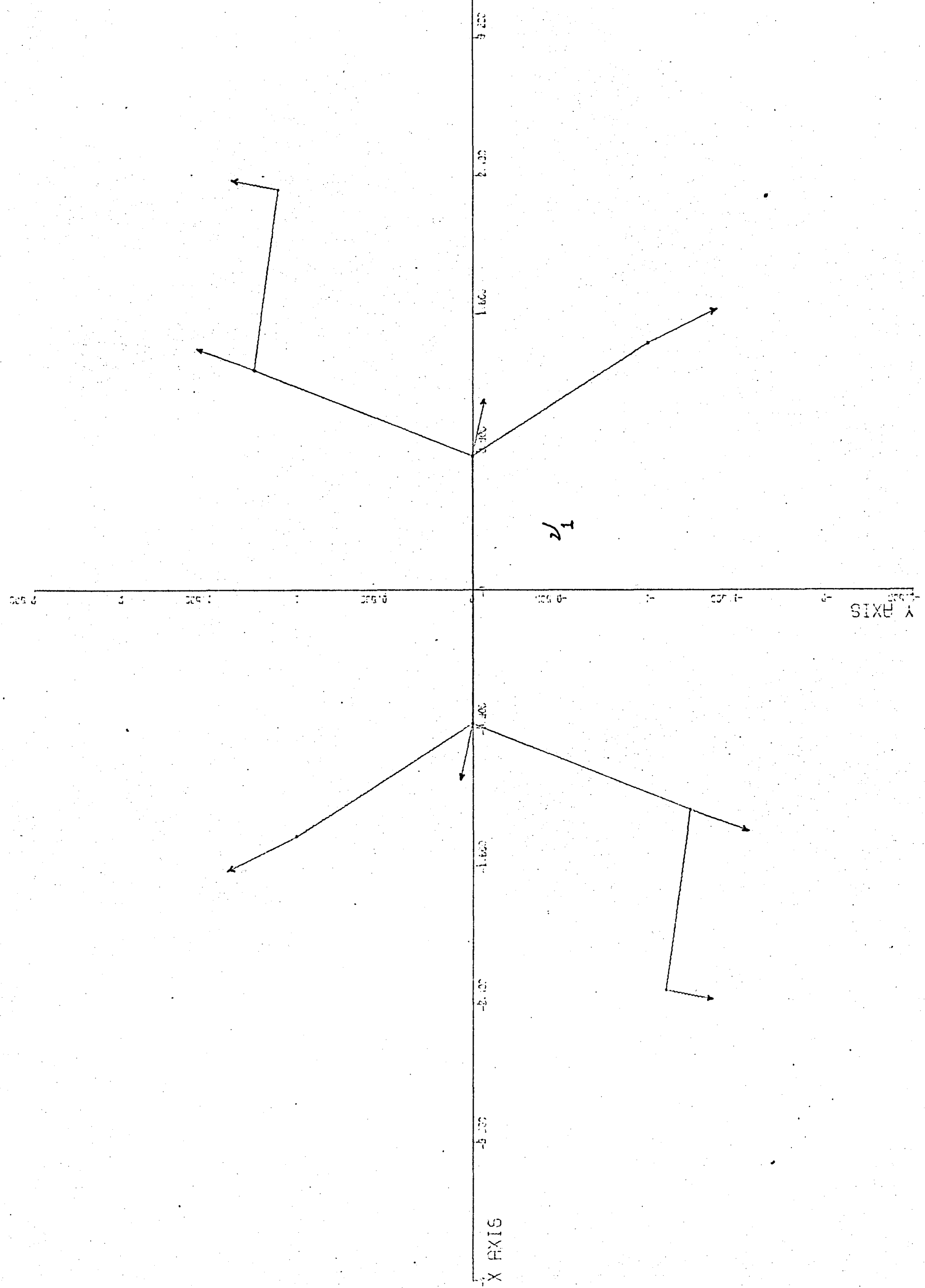




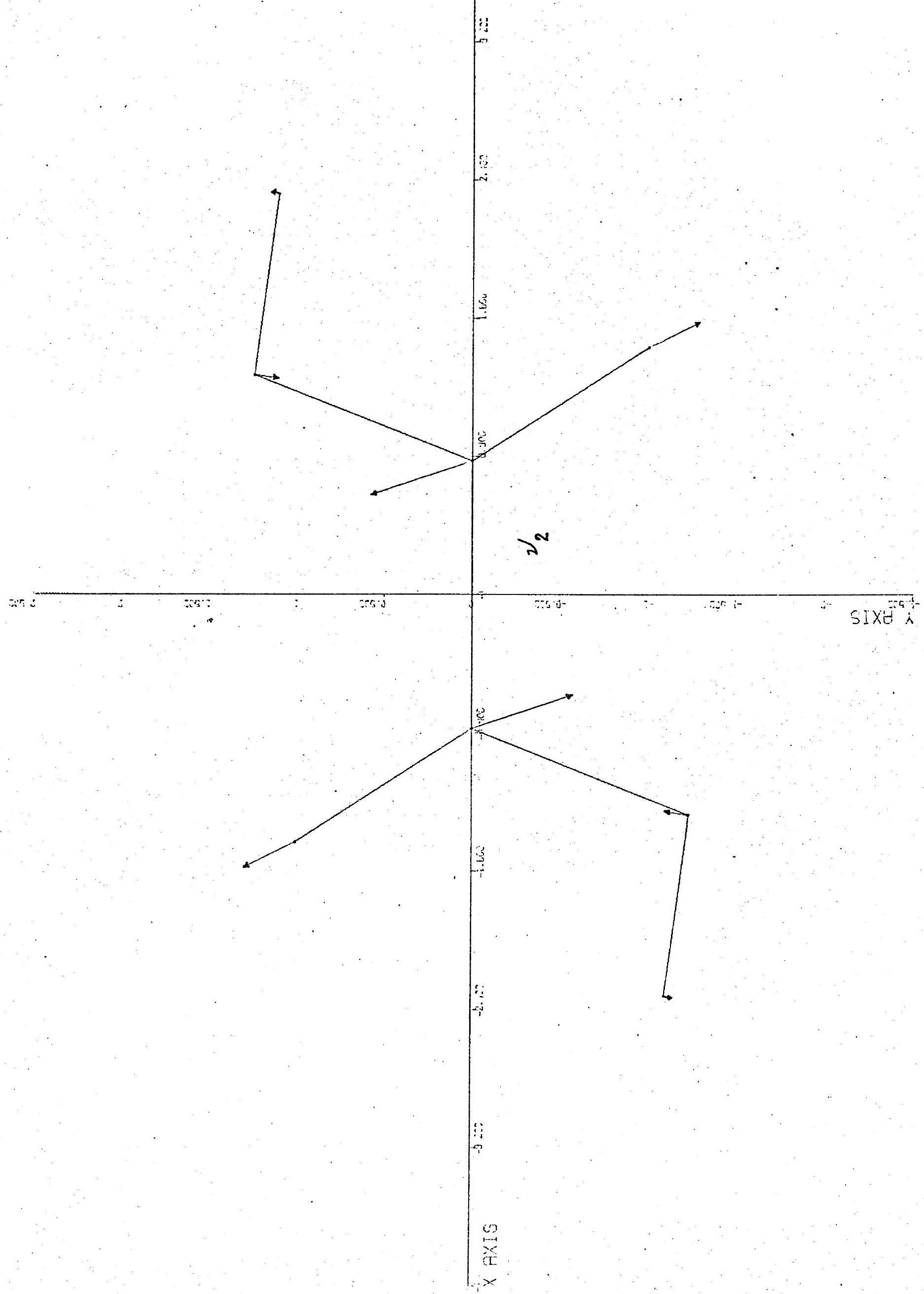




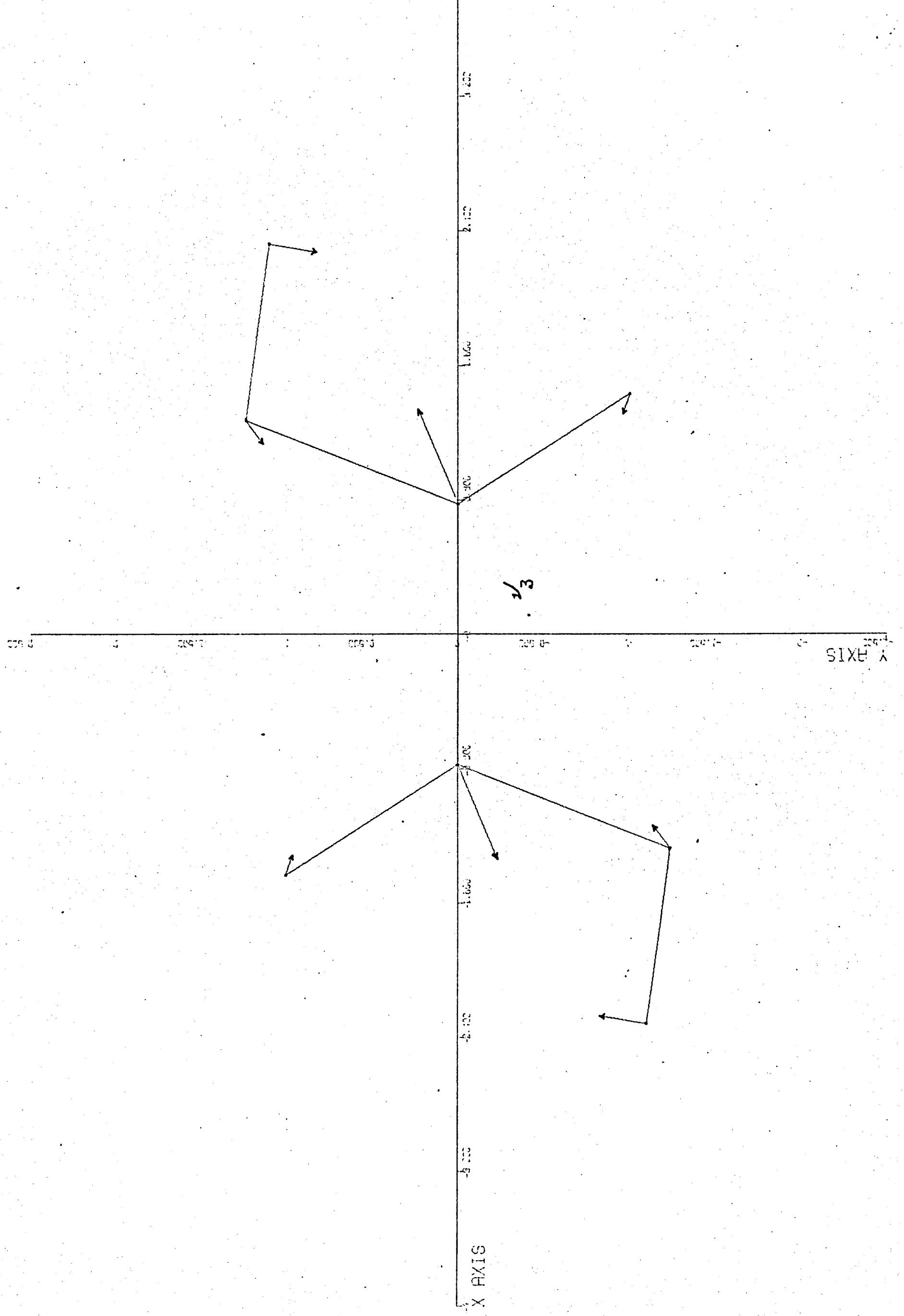




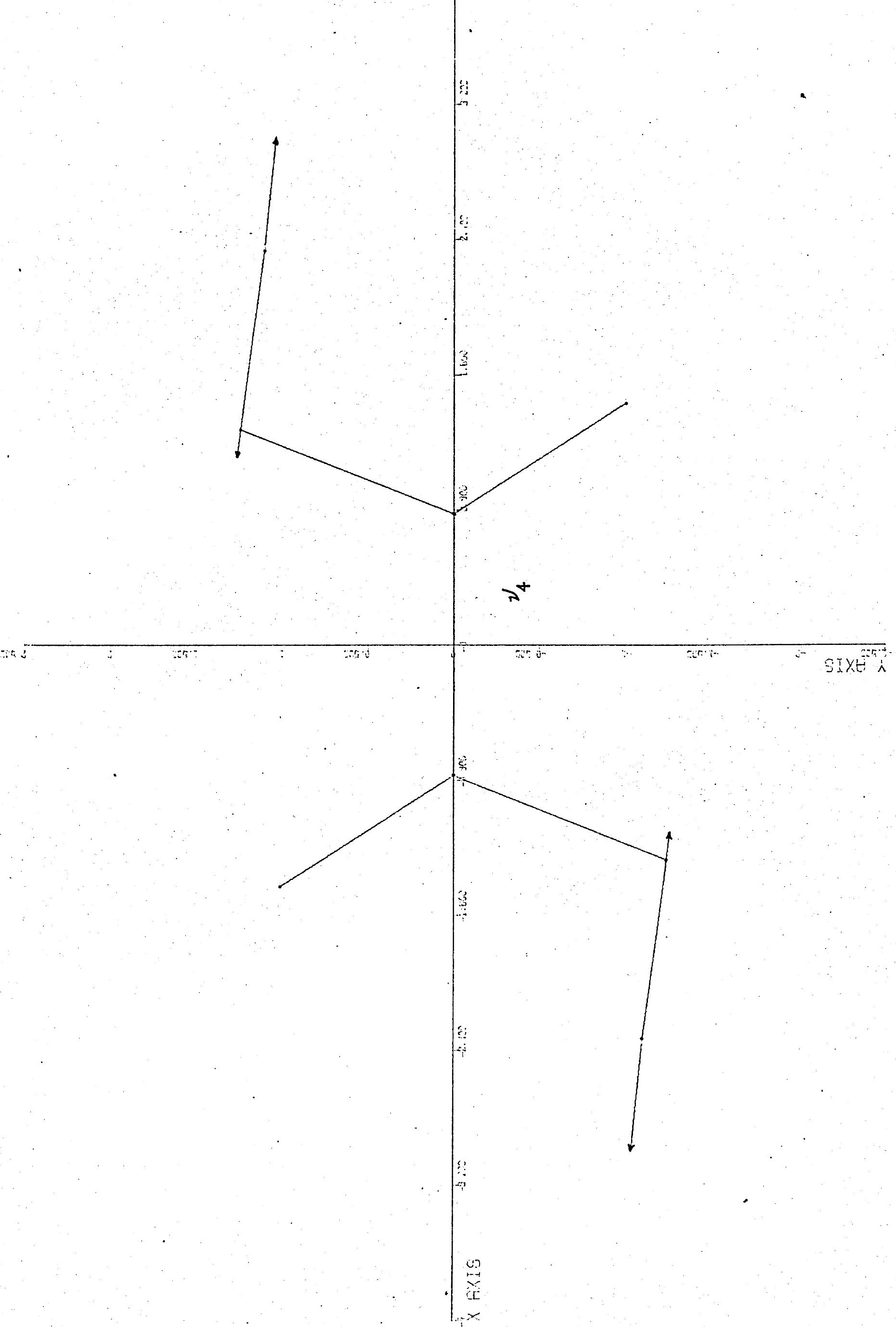




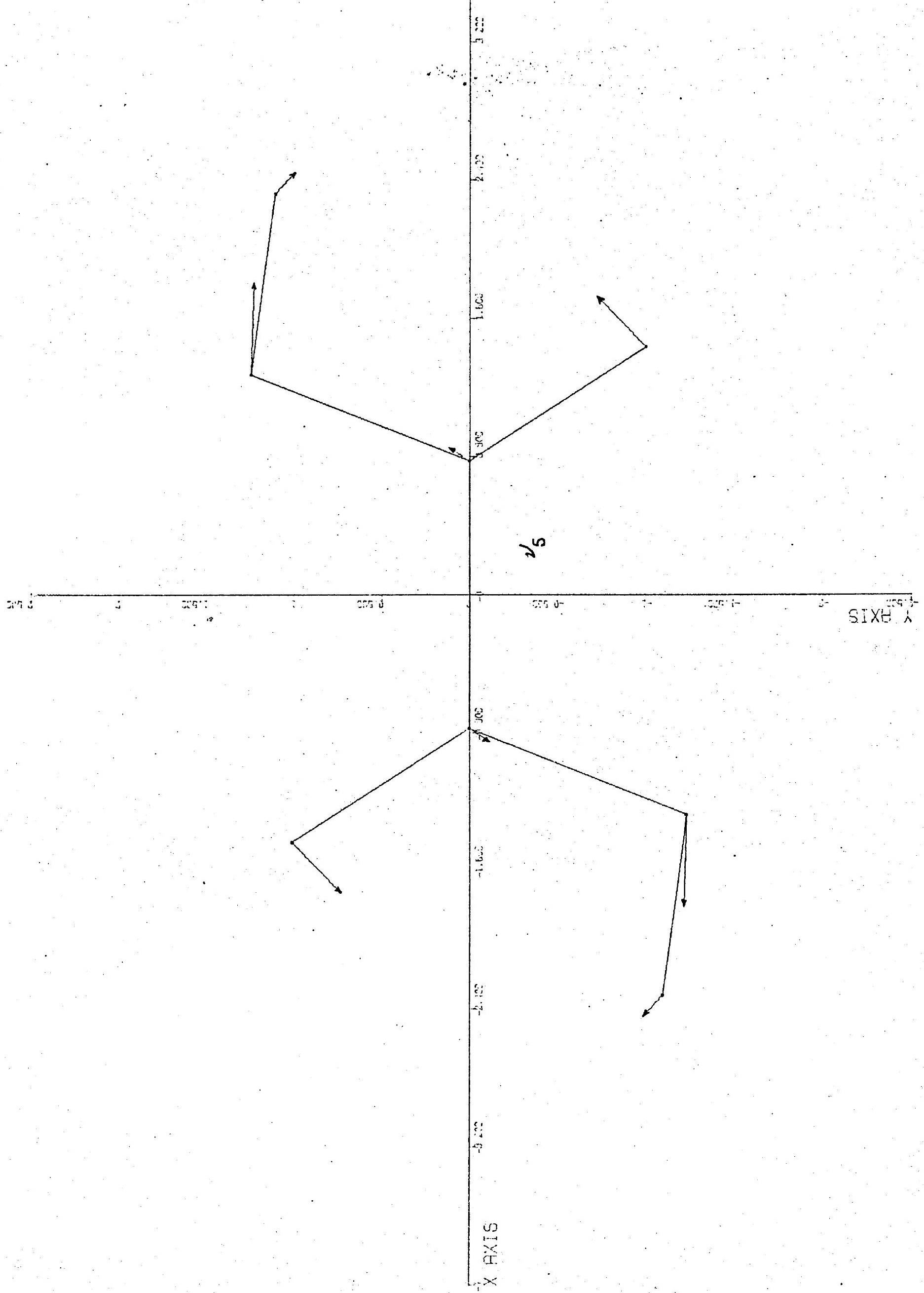




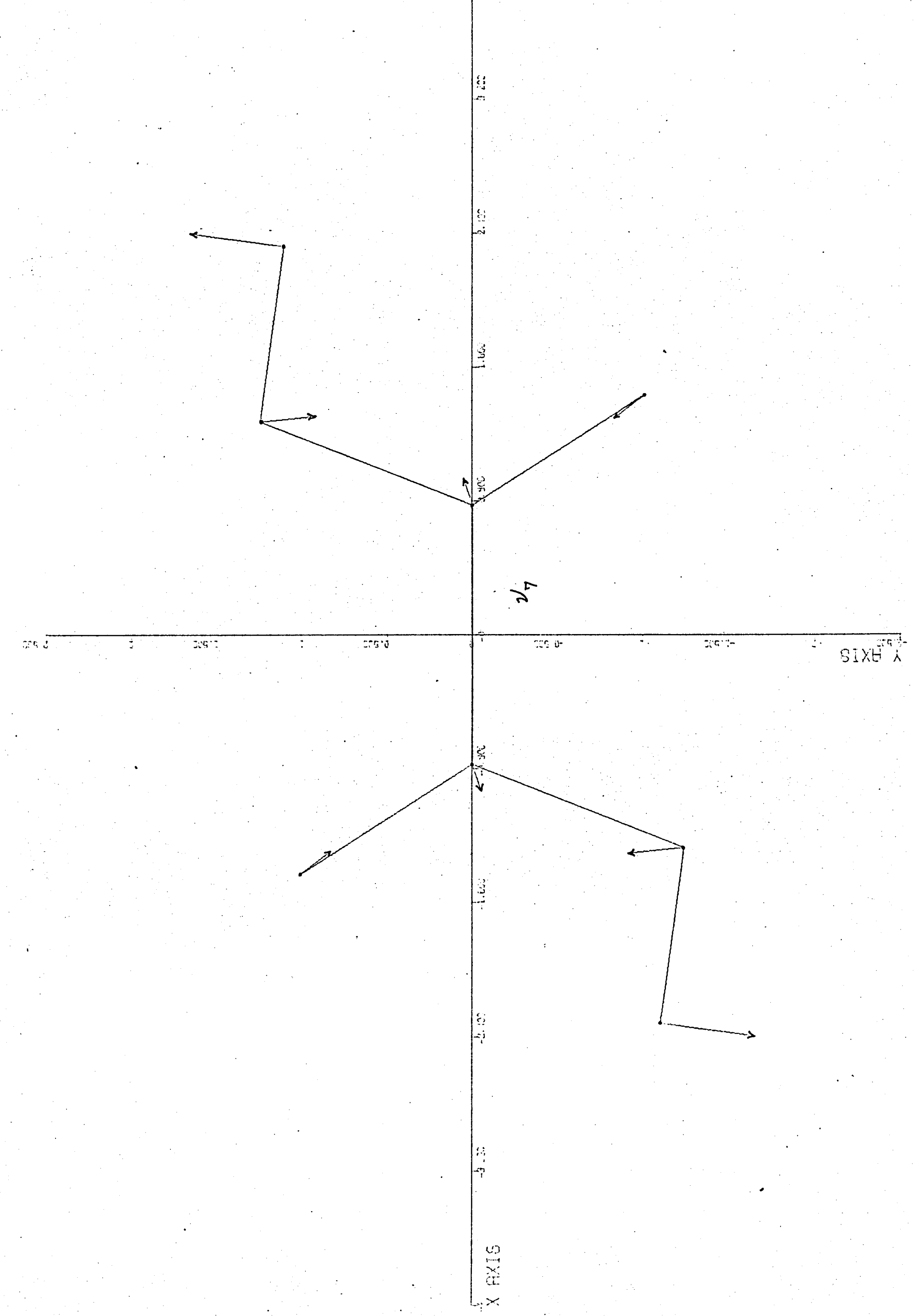




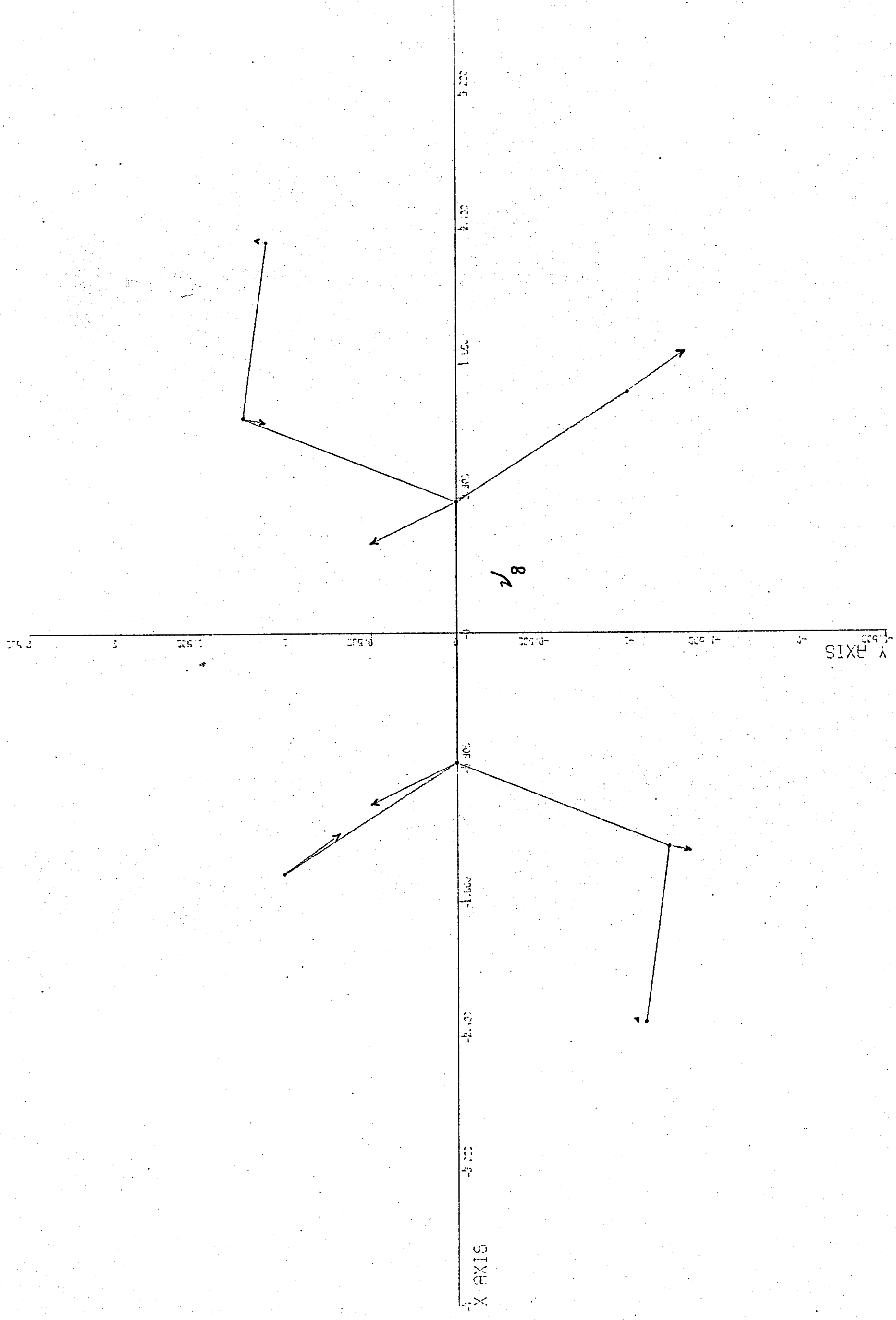




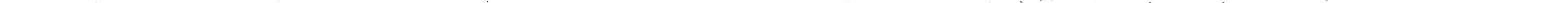




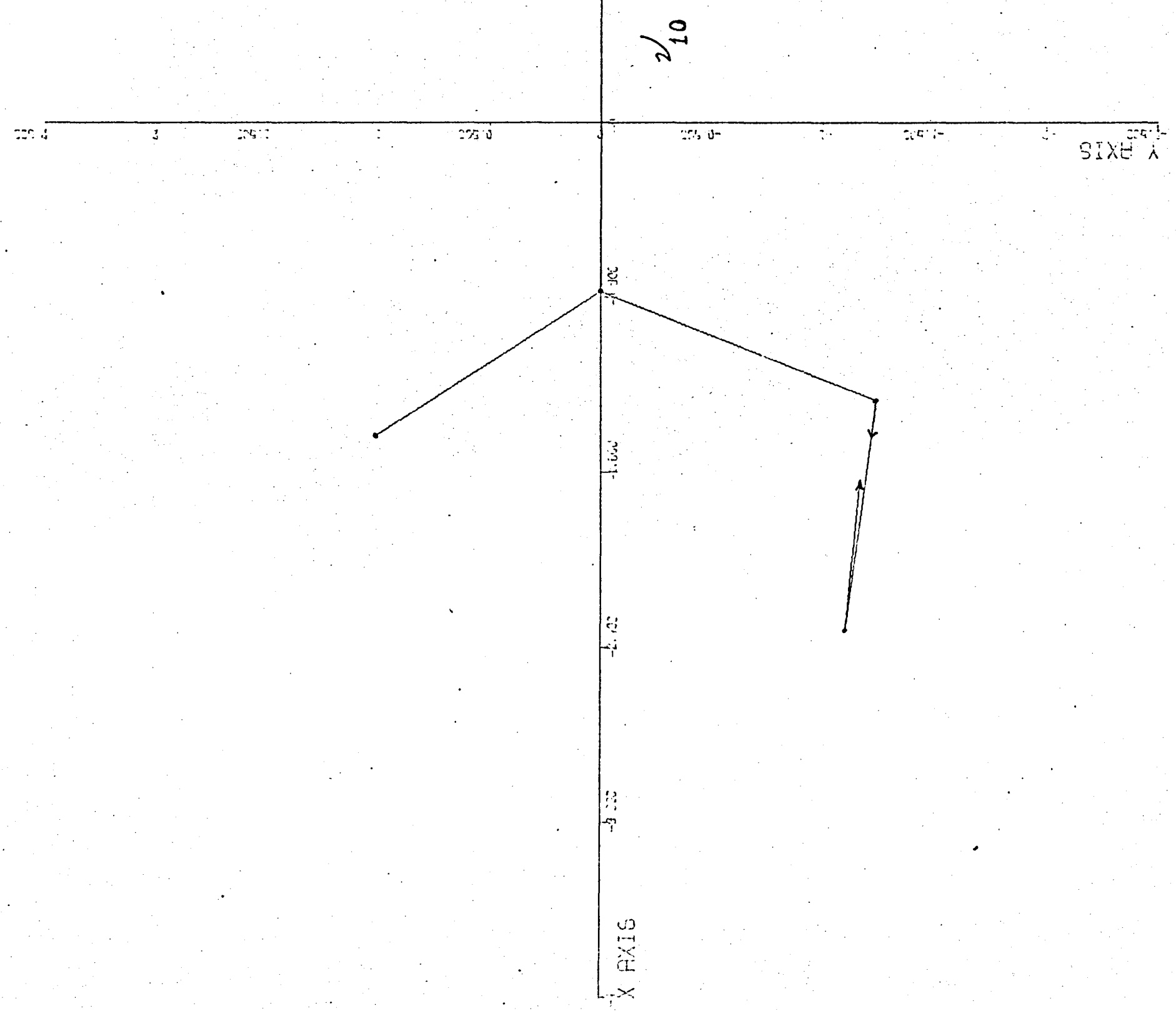




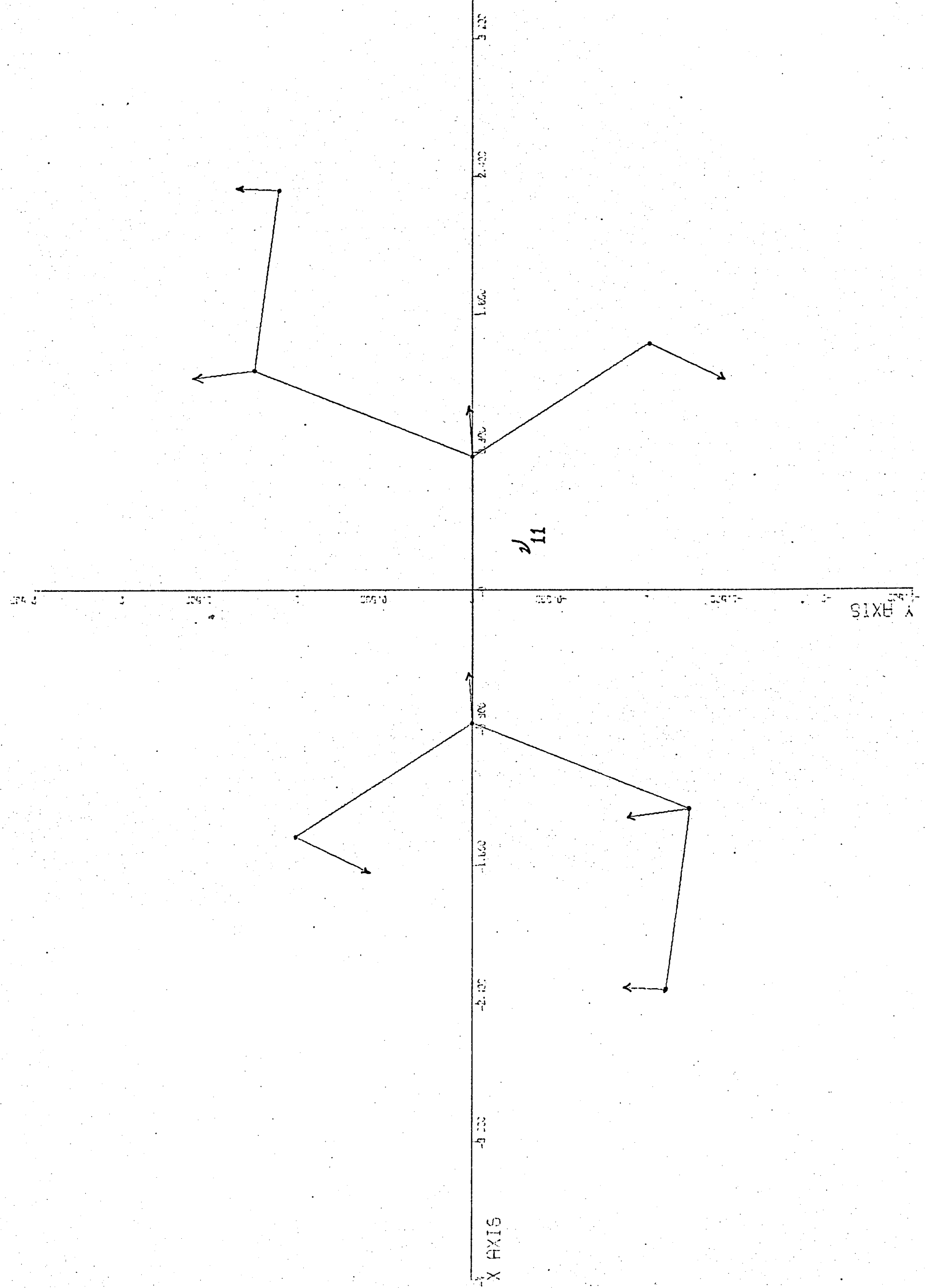




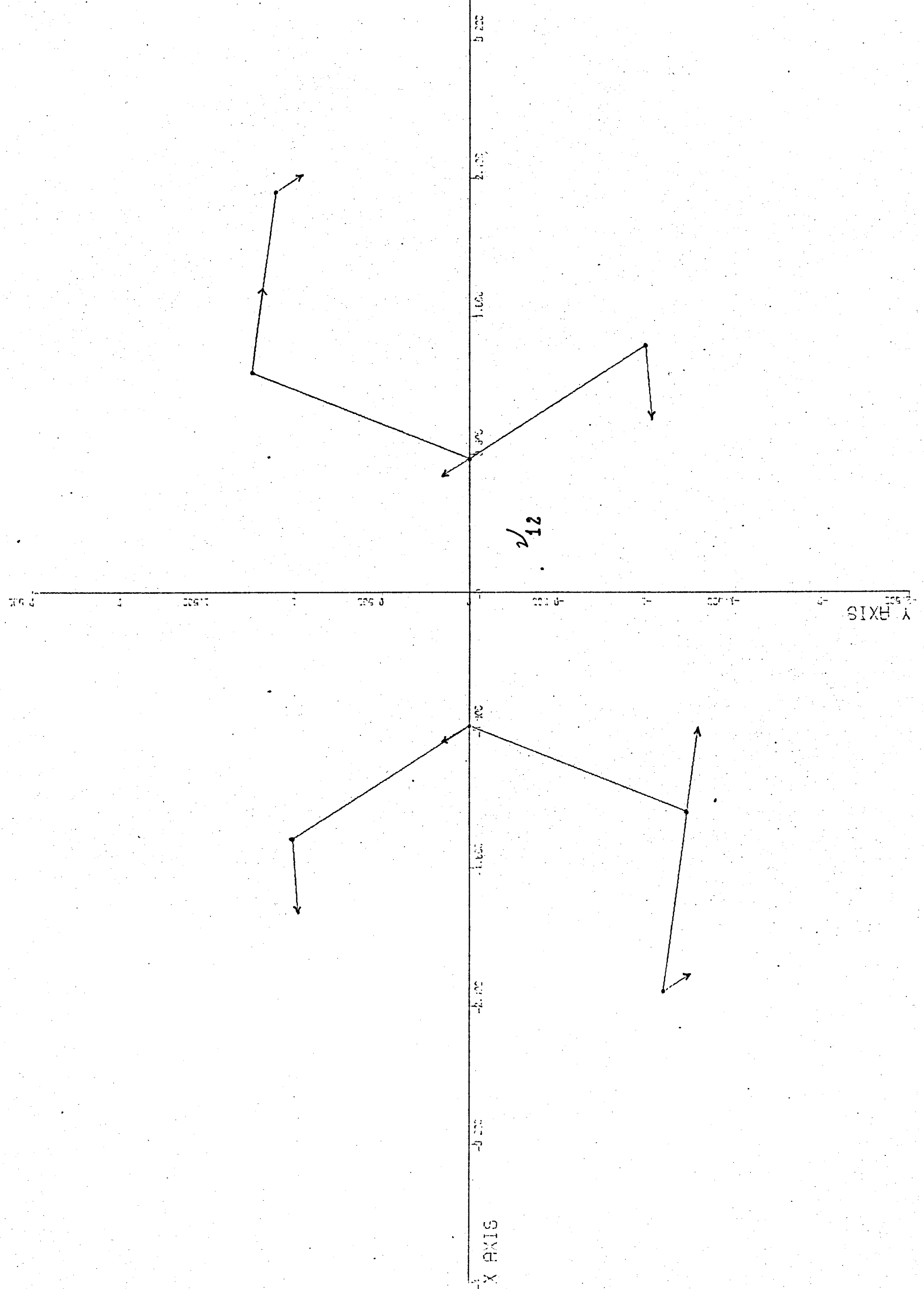




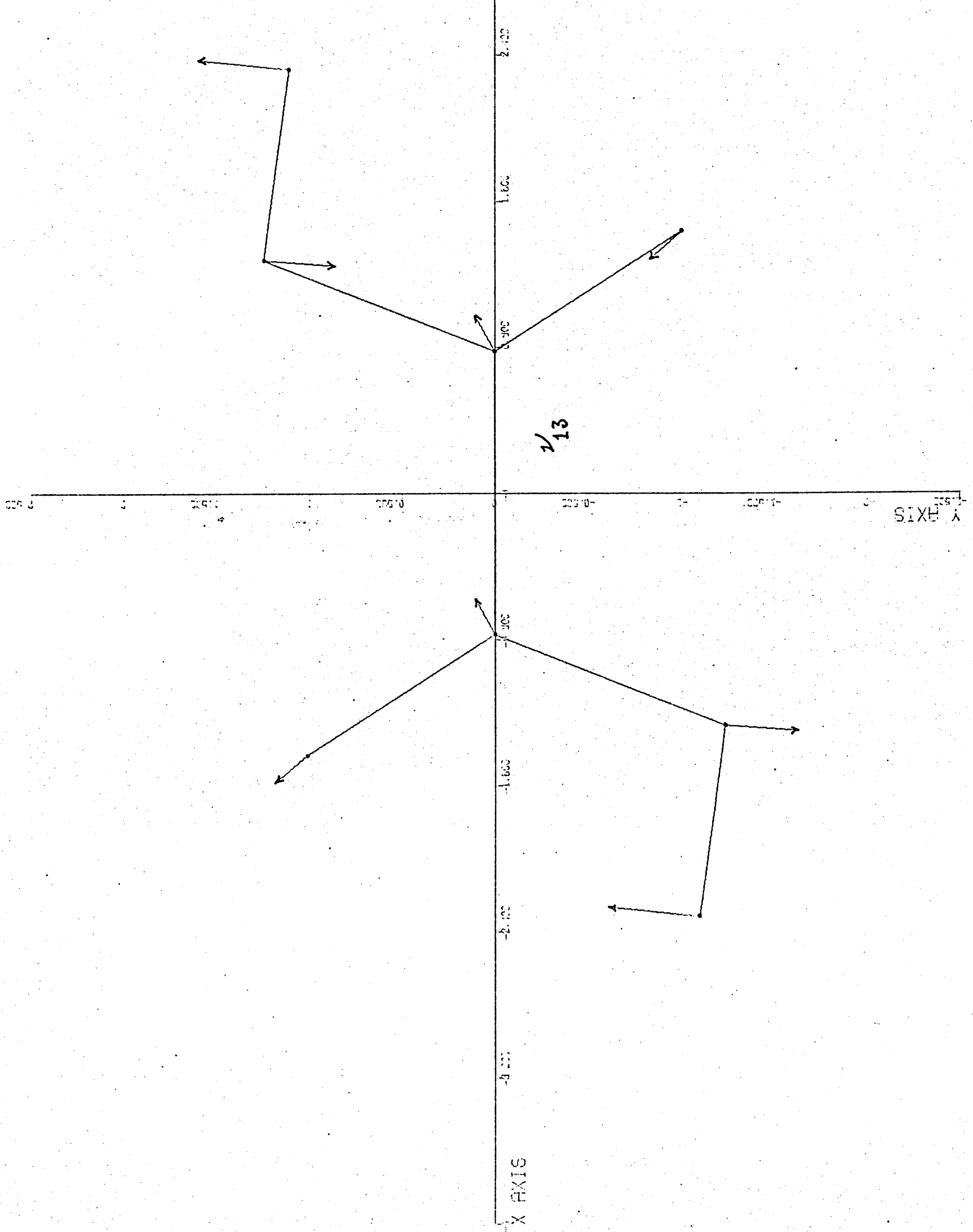


22.4 Calculation using $2 \mathrm{nd}$ mohel

A11 calcialations nore rapeatea using $G$ and $F$ matrices for gaseous oxalic acid and molel which have been constructed in Cinpters 17 and 18 respoctively.

The Jacobian matrix is given in Table 30 . Table 28 shous 4 sets of force constants $A, B, C, D$, used to celculate the corresionding sets of the calculated frequencies in Table 29.

Table 28

Sets of Forco Constants

\begin{tabular}{|c|c|c|c|c|}
\hline \multirow{2}{*}{$\begin{array}{l}\text { Type of } \\
\text { force constant }\end{array}$} & \multicolumn{4}{|c|}{ Foree constants $\left(\mathrm{mu} / \mathrm{A}^{\circ}\right)$} \\
\hline & Set $A$ & $\operatorname{set} B$ & Sot $C$ & Set $D$ \\
\hline$K_{C C}$ & 3.2 & 3.2 & 3.2 & 2.8 \\
\hline $\mathrm{x}_{\mathrm{C}}=\mathrm{O}$ & 10.5 & 10.5 & 20.5 & 10.9 \\
\hline$k_{C} \rightarrow 0$ & 6.5 & 6.5 & 6.5 & 6.5 \\
\hline$x_{0-i}$ & 6.5 & 6.5 & 6.5 & 6.5 \\
\hline$h_{0 . . . h}$ & 0.45 & 0.45 & 0.45 & 0.45 \\
\hline${ }^{\mathrm{H}} \mathrm{C}-\mathrm{CoO}$ & 0.45 & 0.15 & 0.45 & 0.45 \\
\hline${ }^{H_{C}-C-O}$ & 0.3 & 0.3 & 0.3 & 0.3 \\
\hline $\mathrm{H}-\mathrm{COO}$ & 0.05 & 0.2 & 0.1 & 0.1 \\
\hline $\mathrm{H}_{\mathrm{C}-\mathrm{O}-\mathrm{H})}$ & 0.85 & 0.35 & 0.85 & 0.85 \\
\hline$H_{0-3 . . .0}$ & 0.02 & 0.02 & 0.02 & 0.02 \\
\hline$H_{13} \ldots . O=C$ & 0.01 & 0.01 & 0.01 & 0.01 \\
\hline $\mathrm{F}_{\mathrm{C}_{1} \mathrm{O}_{3}}=\mathrm{F}_{\mathrm{C}_{2} \mathrm{O}_{6}}$ & 1.2 & 1.2 & 1.2 & 1.2 \\
\hline $\mathrm{F}_{\mathrm{C}_{1} \mathrm{O}_{4}}=\mathrm{E}_{\mathrm{C}_{2} \mathrm{O}_{7}}$ & 0.8 & 0.8 & 0.8 & 0.8 \\
\hline
\end{tabular}


Table 29

Sets of calculated frequencies

\begin{tabular}{|c|c|c|c|c|c|}
\hline \multirow{2}{*}{\multicolumn{2}{|c|}{$\begin{array}{l}\text { Observed trequencies } \\
\qquad\left(\mathrm{cm}^{-1}\right)\end{array}$}} & \multicolumn{4}{|c|}{ Calculatcd frequencies $\left(\mathrm{cm}^{-1}\right)$} \\
\hline & & Sot $A$ & Set $B$ & Set C & $\operatorname{set} D$ \\
\hline \multicolumn{6}{|c|}{ Ag species } \\
\hline$v_{1}$ & 812 & 830.6 & 832.9 & 832.1 & 820.95 \\
\hline$v_{2}$ & 1800 & 1785.7 & 1789.6 & 1789.3 & 1810.90 \\
\hline$v_{3}$ & 1423 & 1420.8 & 1426.5 & 1424.6 & 1409.56 \\
\hline$v_{4}$ & - & 3471.8 & 3471.9 & 3471.9 & 3471.85 \\
\hline$v_{5}$ & - & 8.2 & 8.5 & 8.4 & 8.37 \\
\hline$v_{6}$ & 405 & 401.3 & 420.7 & 414.4 & 414.56 \\
\hline$v_{7}$ & 1195 & 1256.0 & 1259.8 & 1258.6 & 1246.20 \\
\hline \multicolumn{6}{|c|}{ Bu species } \\
\hline$v_{8}$ & 1812 & 1786.8 & 1787.2 & 1787.0 & 1812.48 \\
\hline$v_{9}$ & 1275 & 1230.7 & 1236.8 & 1234.7 & 1236.99 \\
\hline$v_{10}$ & 3475 & 3471.8 & 3471.9 & 3471.9 & 3471.85 \\
\hline$v_{11}$ & - & 302.0 & 302.1 & 302.0 & 302.03 \\
\hline$v_{12}$ & 460 & 432.0 & 465.0 & 454.4 & 454.91 \\
\hline$v_{13}$ & 1325 & 1323.1 & 1324.2 & 1323.8 & 1324.37 \\
\hline
\end{tabular}

Aeasurements of fit show that force constants in Set $D$ are better than the others. Thus, this set is used to calculate the potential onergy distribution and the nodes of vibration. 
22.5 Assignmant of the observed infrared and Raman bands,

potential onorgy distribution and normal nodes

Assigament of the observed infrared and Reman bands to fit

the calculeted froquencies for gaseous oxalic acid 2 nd godel is given in Tablo $\mathbf{3 1}$

Table 31

\begin{tabular}{|c|c|c|c|c|c|}
\hline \multicolumn{2}{|c|}{$\begin{array}{l}\text { Ouserved frequencies } \\
\qquad\left(\mathrm{cm}^{-1}\right)\end{array}$} & \multicolumn{2}{|c|}{ Assignrnent } & \multirow{2}{*}{$\begin{array}{l}\text { Calculated } \\
\text { frequencles } \\
\left(\mathrm{cm}^{-1}\right)\end{array}$} & \multirow{2}{*}{$\Delta v=v_{\text {calc. }}-v_{\text {obs. }}$} \\
\hline Infrarod & Raman & Mode & Species & & \\
\hline 3560 & & $v_{2}+v_{8}$ & $\mathrm{Bu}$ & - & - \\
\hline 3475 & & $v_{10}$ & $\mathrm{Bu}$ & 3471.9 & 3.1 \\
\hline 1812 & & $v_{8}$ & $\mathrm{Bu}$ & 1812.5 & 0.5 \\
\hline 1325 & & $v_{13}$ & $\mathrm{Bu}$ & 1324.4 & -0.6 \\
\hline 1275 & & $v_{9}$ & Bu & 1237.0 & -38.0 \\
\hline 660 & & & $\mathrm{Au}$ & & \\
\hline $620^{\circ}$ & & $\begin{array}{l}\text { conbin } \\
\text { band }\end{array}$ & tion & & \\
\hline 460 & & $v_{12}$ & Du & 454.9 & -5.1 \\
\hline & 1800 & $v_{2}$ & $\mathrm{Ag}$ & 1810.9 & 10.9 \\
\hline & 1423 & $v_{3}$ & $A g$ & 1400.6 & -13.4 \\
\hline & 1195 & $v_{7}$ & $\mathrm{Ag}$ & 1246.2 & 51.2 \\
\hline & 812 & $v_{1}$ & $\mathrm{Ag}$ & 821.0 & 9.0 \\
\hline & 405 & $v_{6}$ & $\mathrm{Ag}$ & 414.6 & 9.6 \\
\hline
\end{tabular}

20 The band at $620 \mathrm{~cm}^{-1}$ overleps the bend at $660 \mathrm{~cm}^{-1}$ which was assigned to the out of plane fundaristal, Au species. If the 
$620 \mathrm{~cm}^{-1}$ band was of species Au it would interact with the $660 \mathrm{~cm}^{-1}$. Au fundamental. As this has not occurred it was assigned to the Bu species. This band can not be fitted to the calculated valus for the inplene fundamentals, and thus it was assigned to combination band. There are tro possibilities:

(a) It could be $\left(v_{6}+v_{11}\right)$ in which the sum of both calculated values is $716.6\left(\mathrm{~cm}^{-1}\right)$. As the value for the antmoaicity shift is too high, the other possibility is preferable.

(b) It colld be the combination of two of the out of plane fundarentals with different species $(A \mathrm{u} \times \mathrm{Bg}=\mathrm{Bu})$. The attempt to fit this band as a differonce band was also tried but no suitable pair of fundamentals are bnow.

Potential energy distribution

The potential energy distribution for the planar vibrations for gascous oxalic acid 2nd rodel was calculated and tho rosults are in Table 32 . 

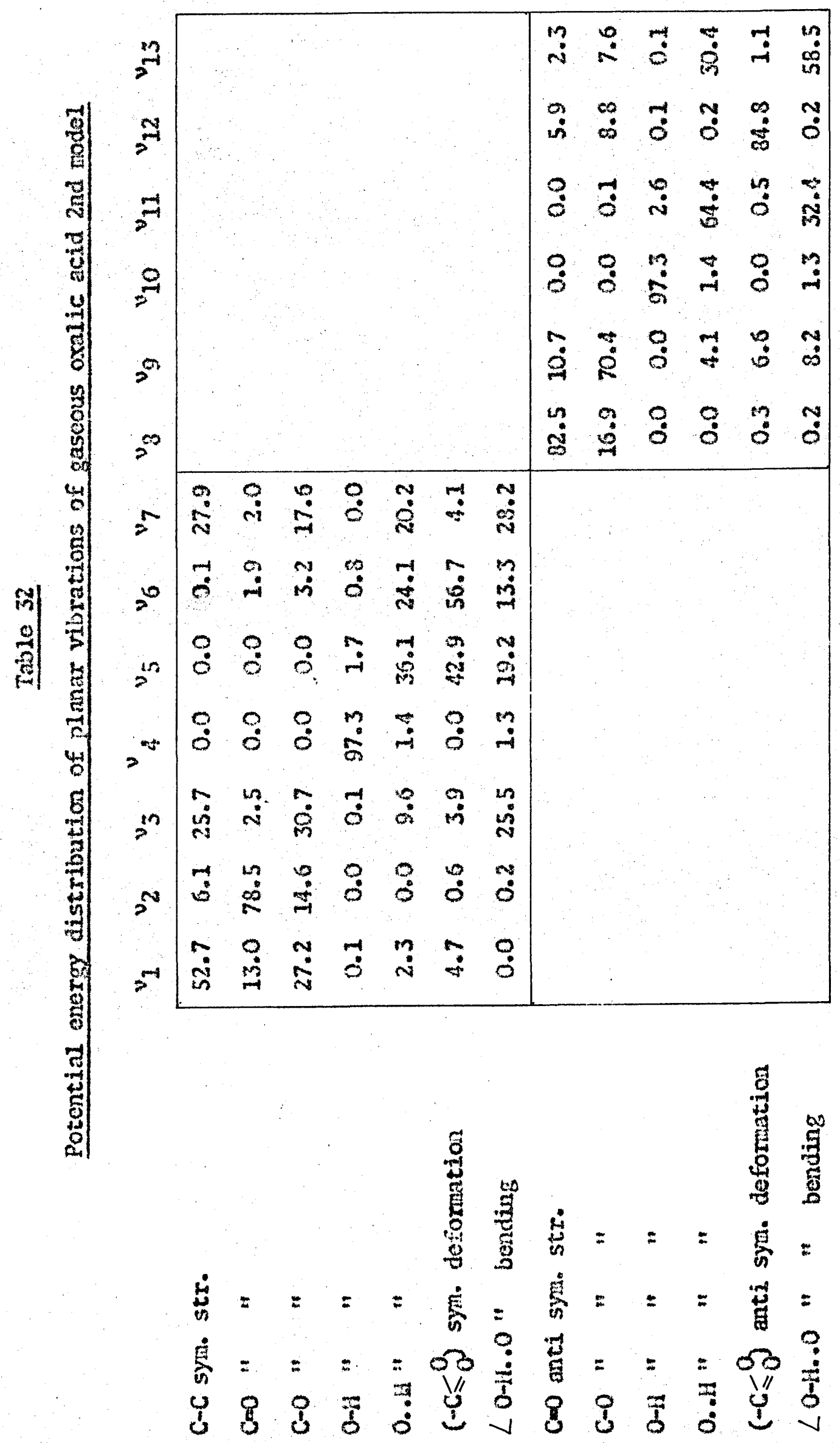
Wodos of vibration

The \& vectors in mass adjusted displacement co-ordinates for gaseous oxalic acid and molel were calculated and 13 inplane modes of vibratioa were plotted by the computer as in the following figuros. 


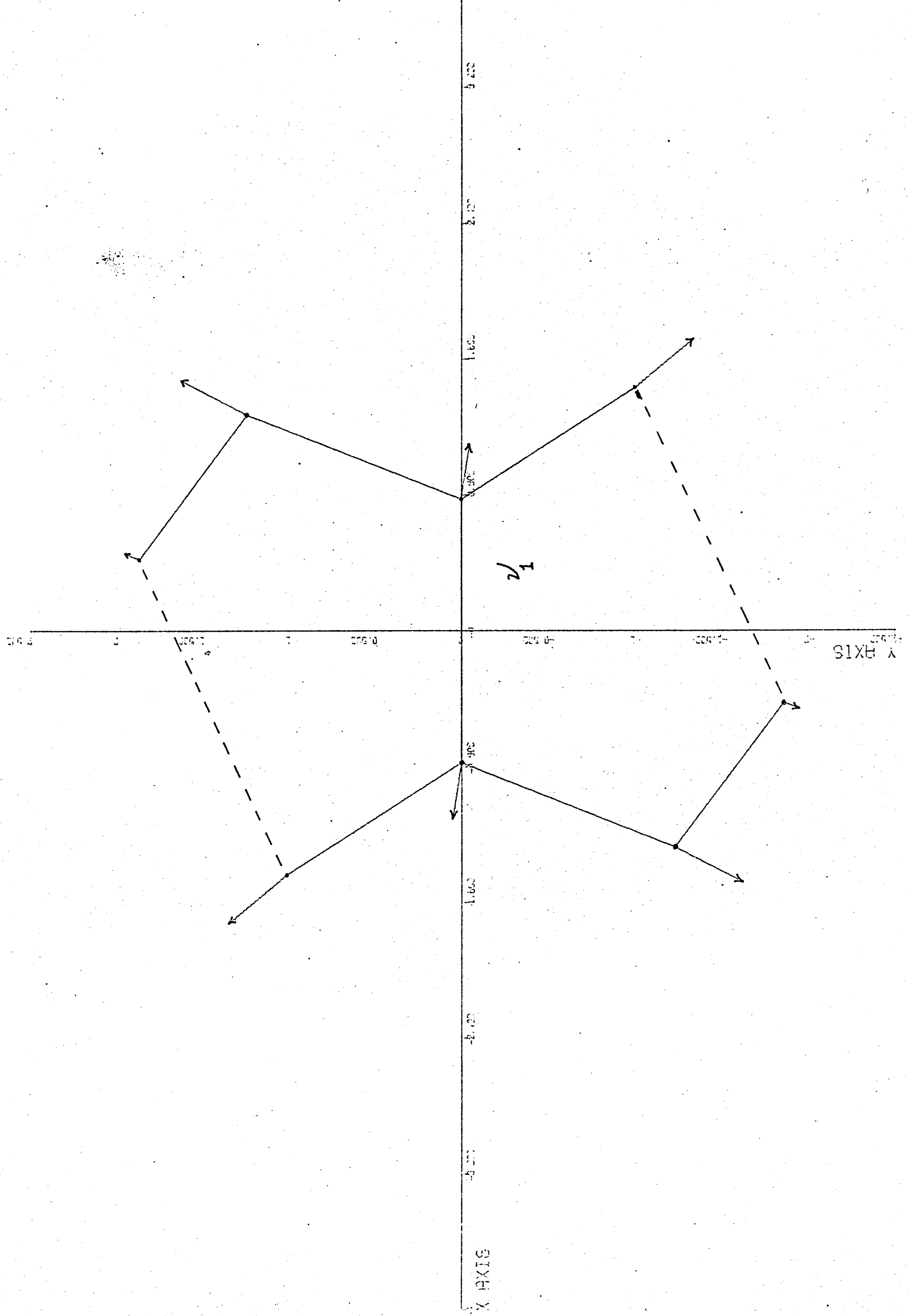




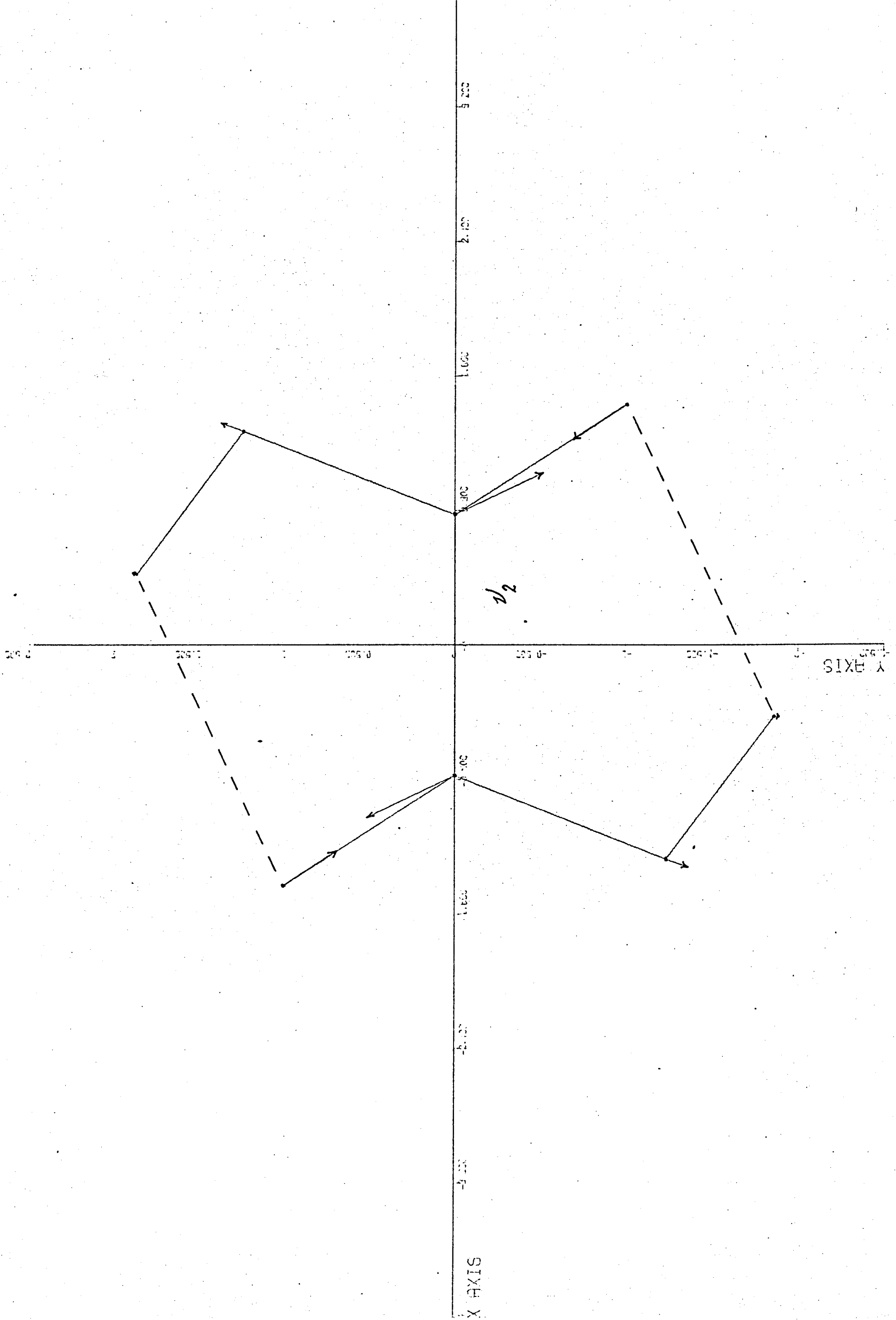




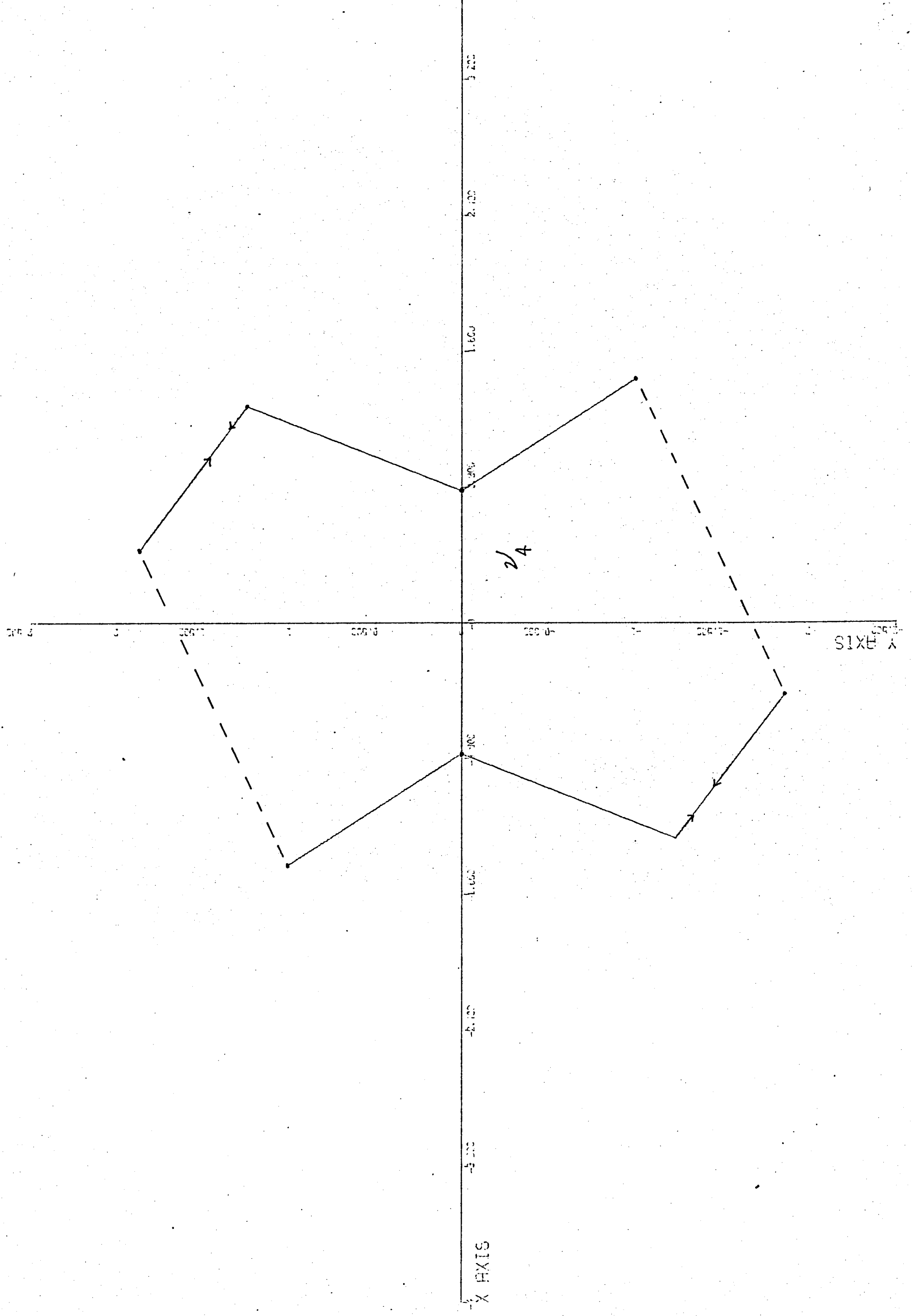




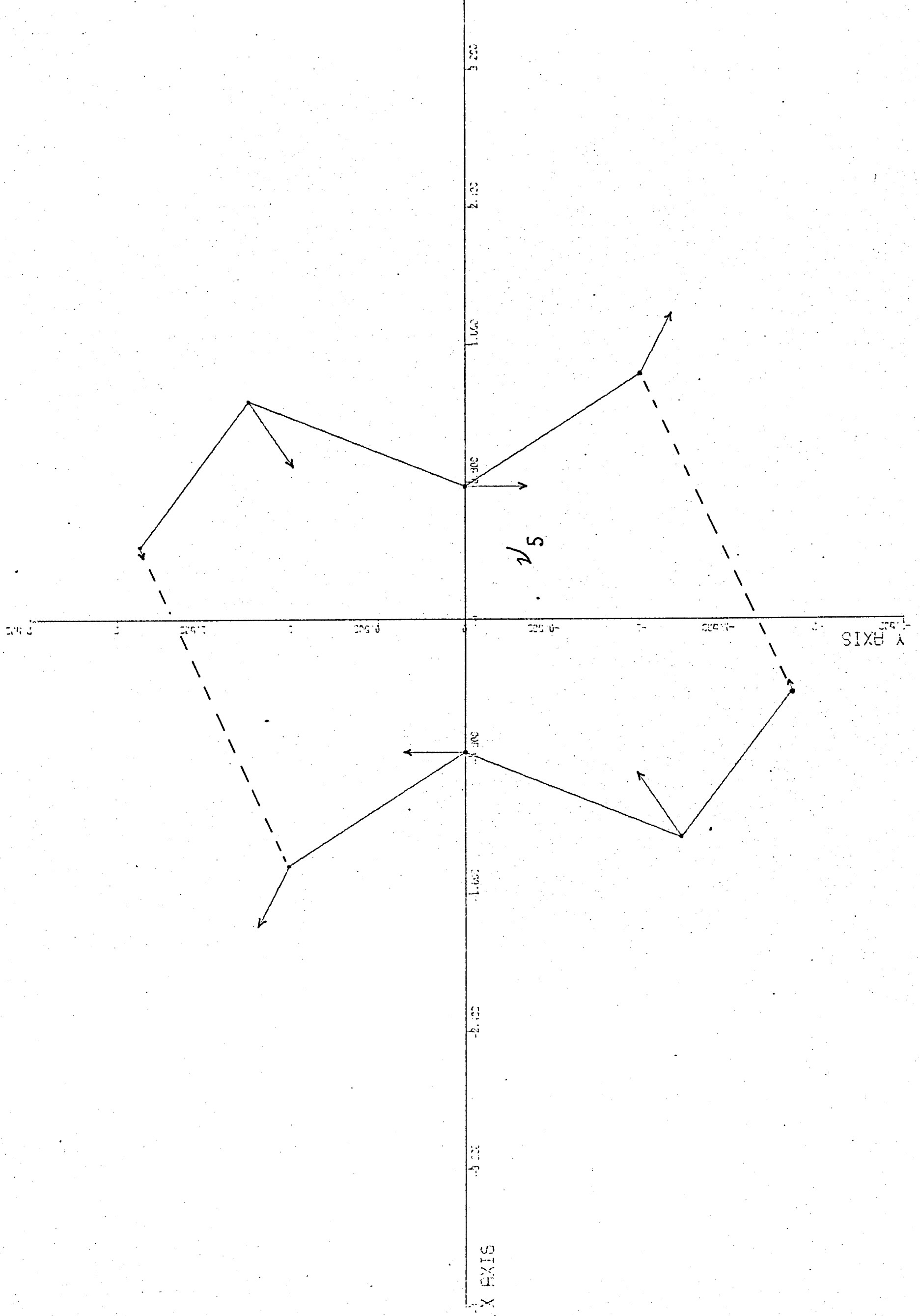




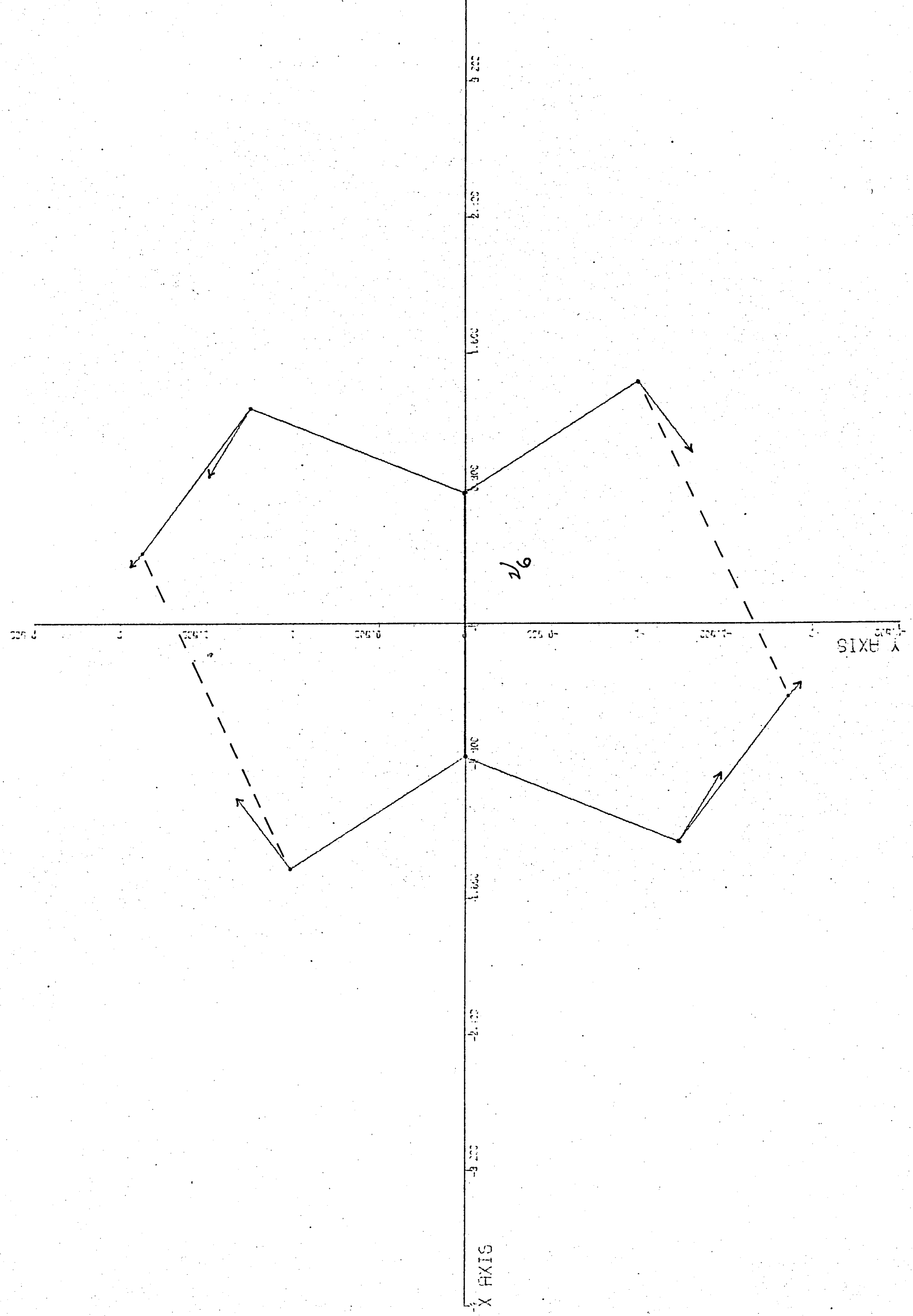




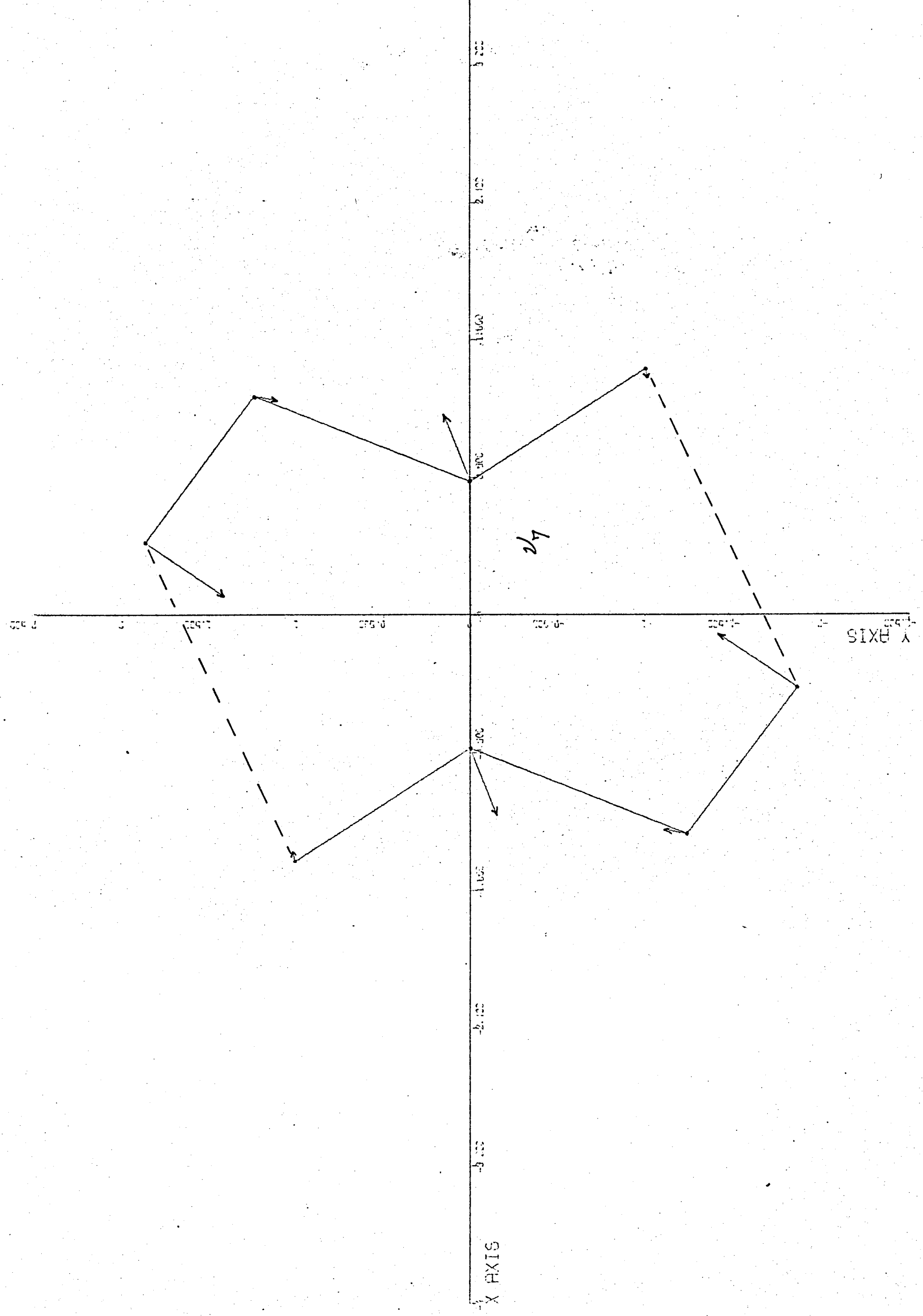




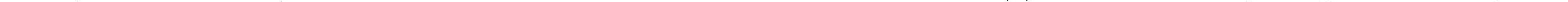




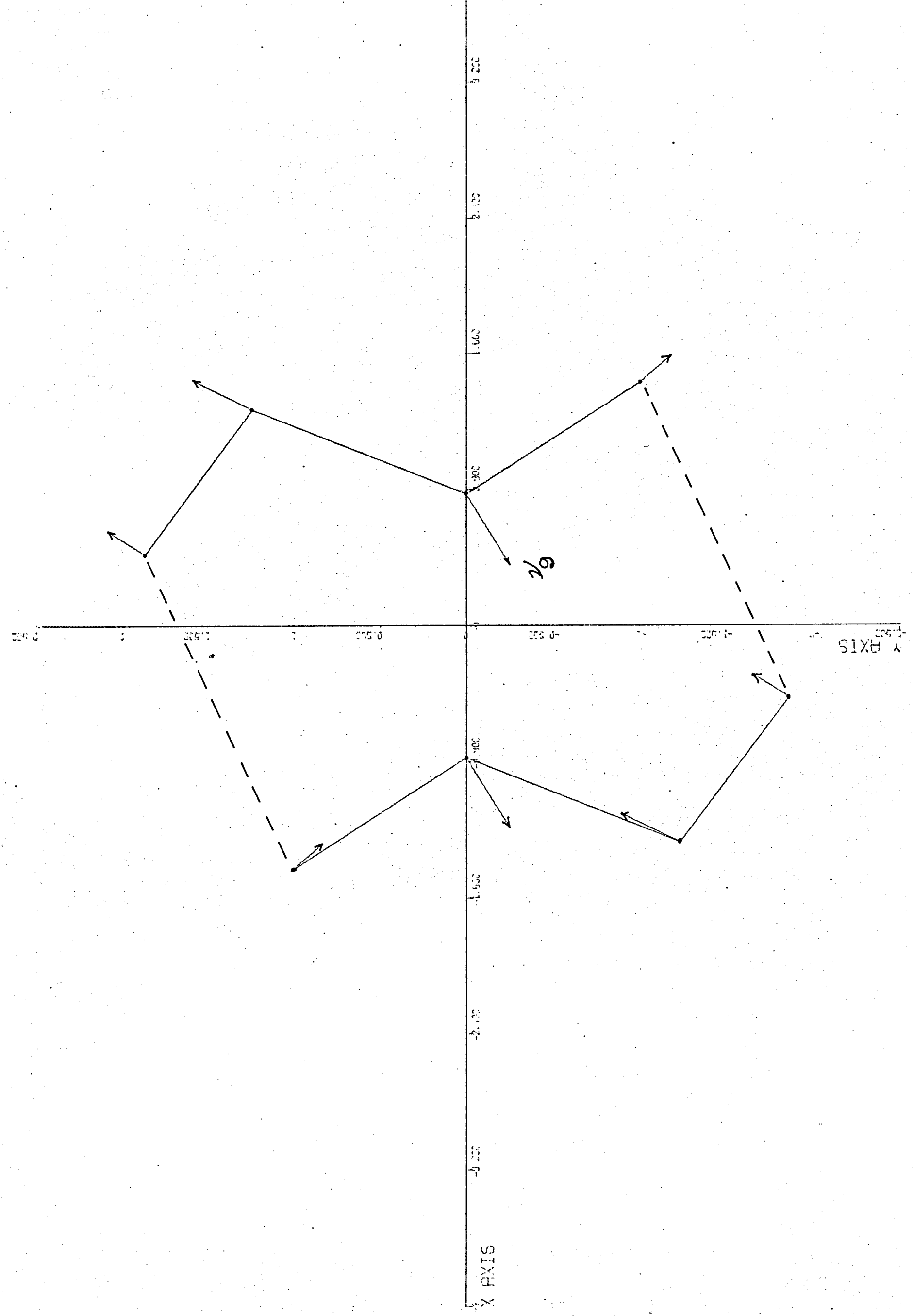




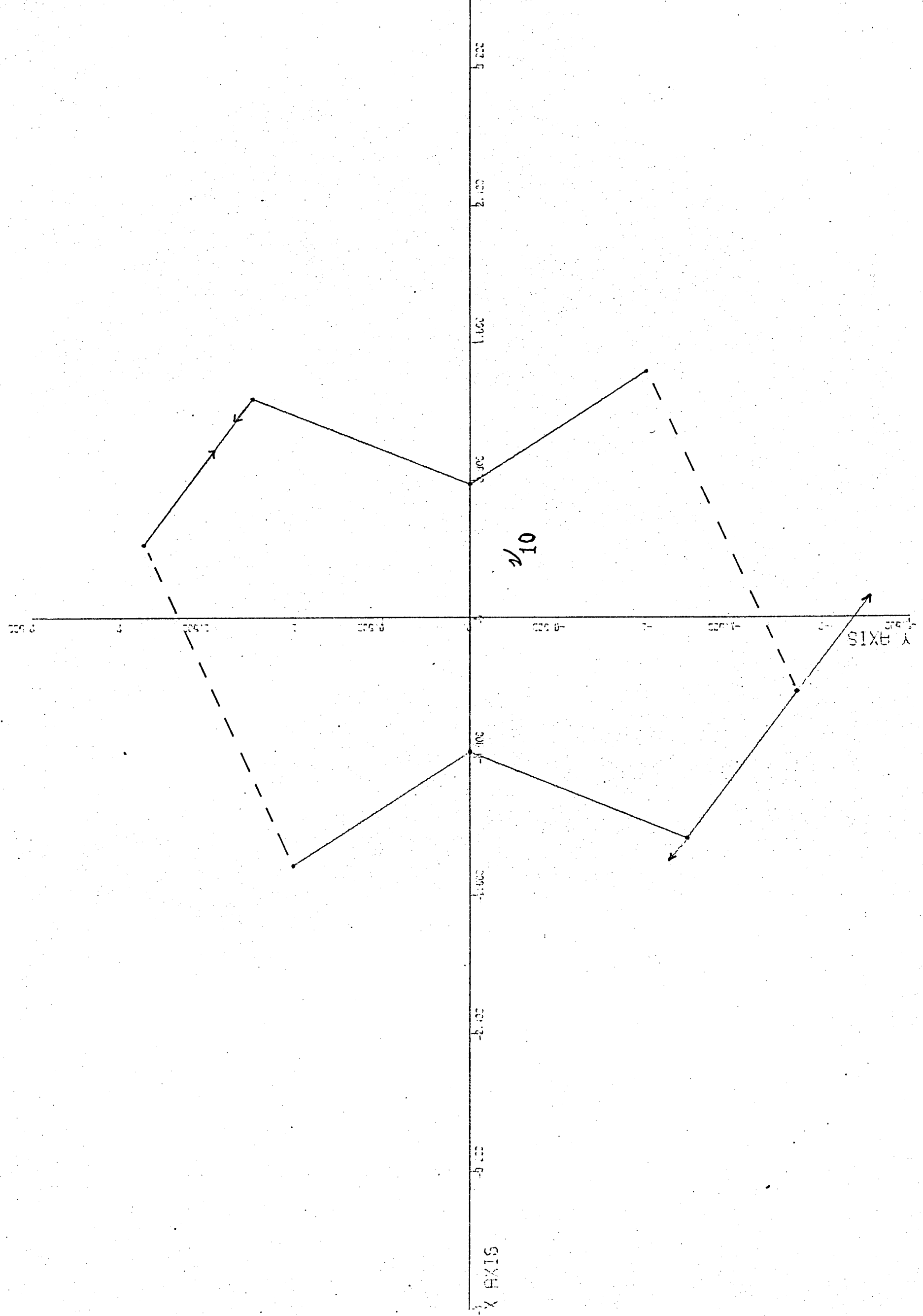




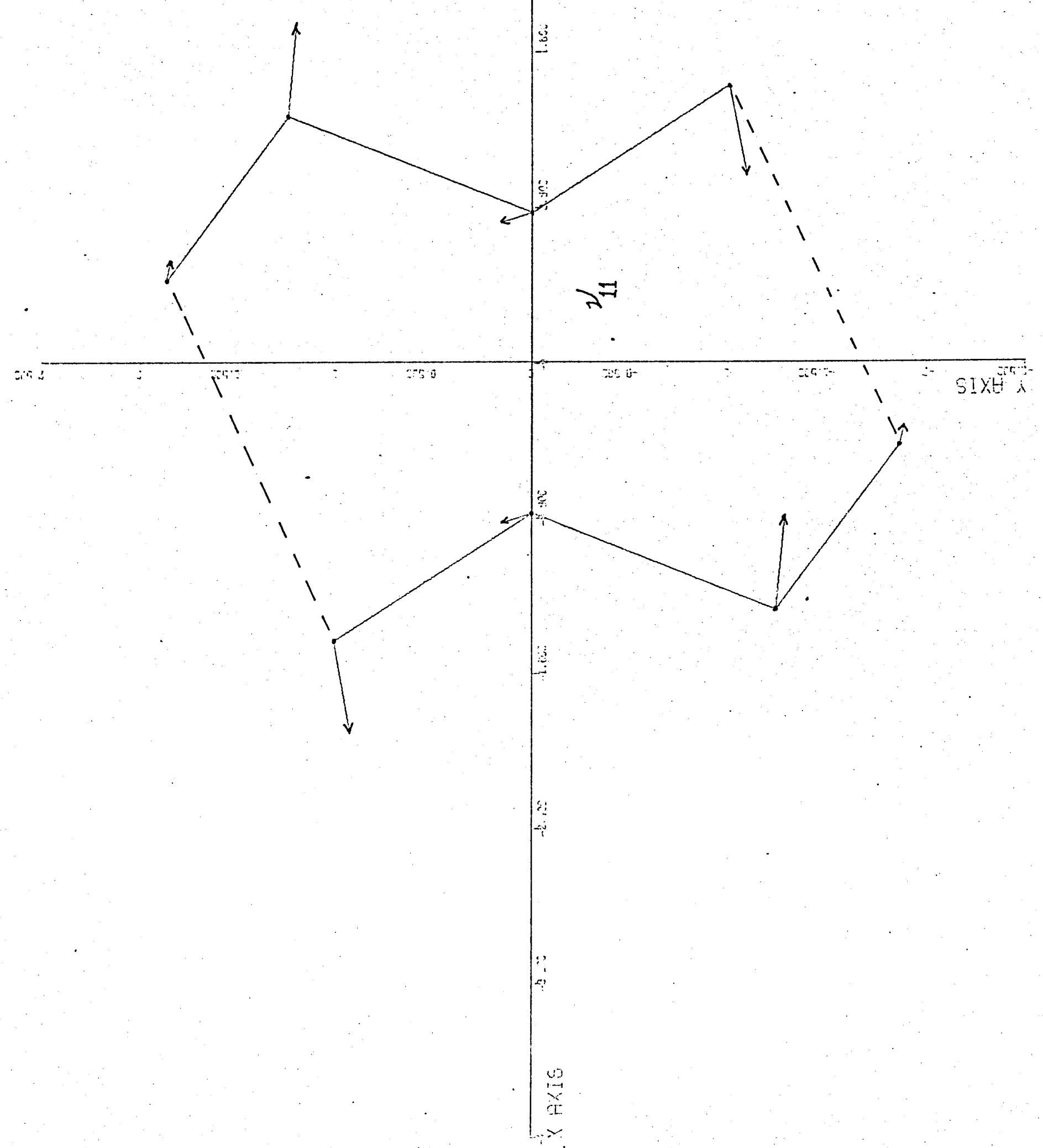




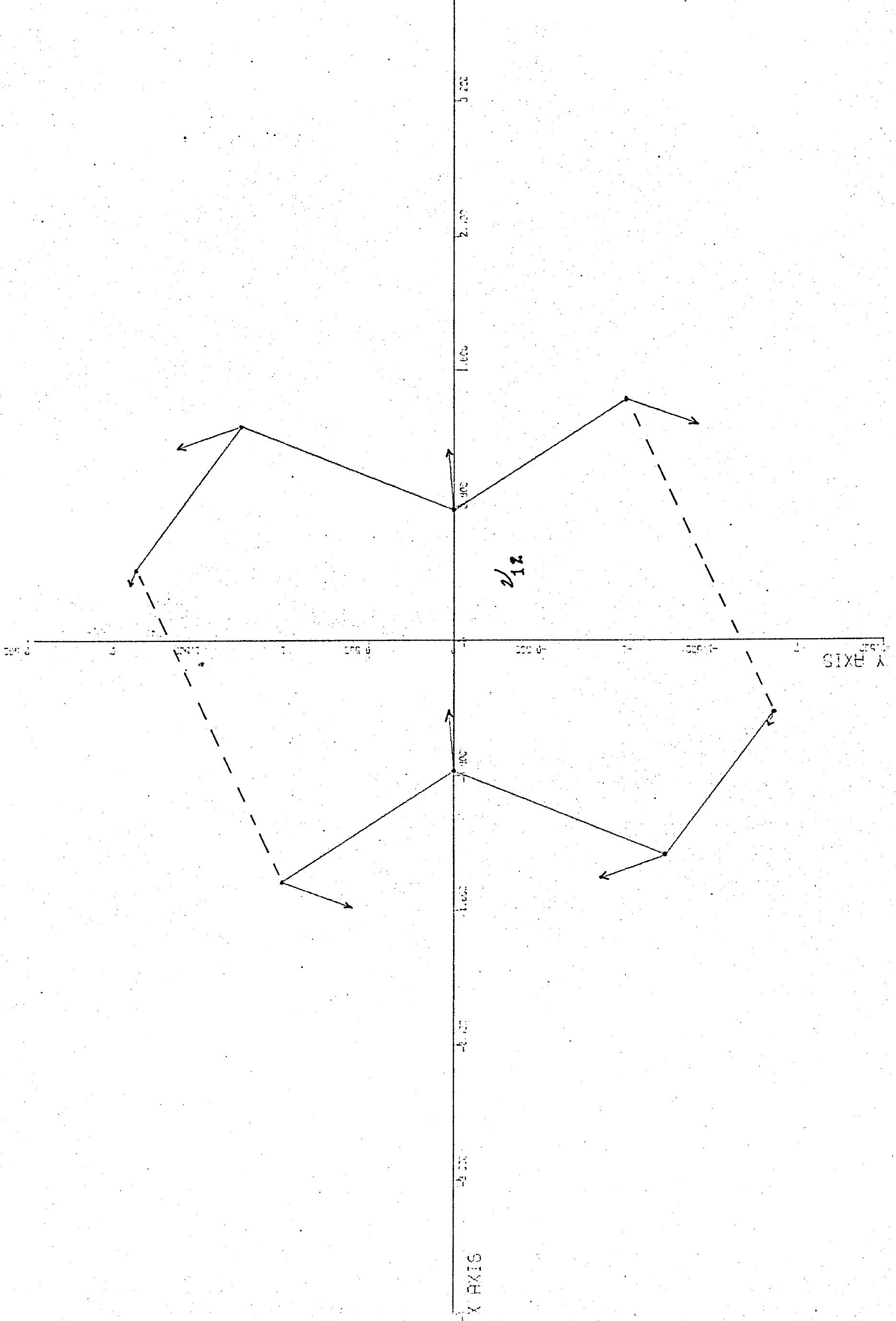




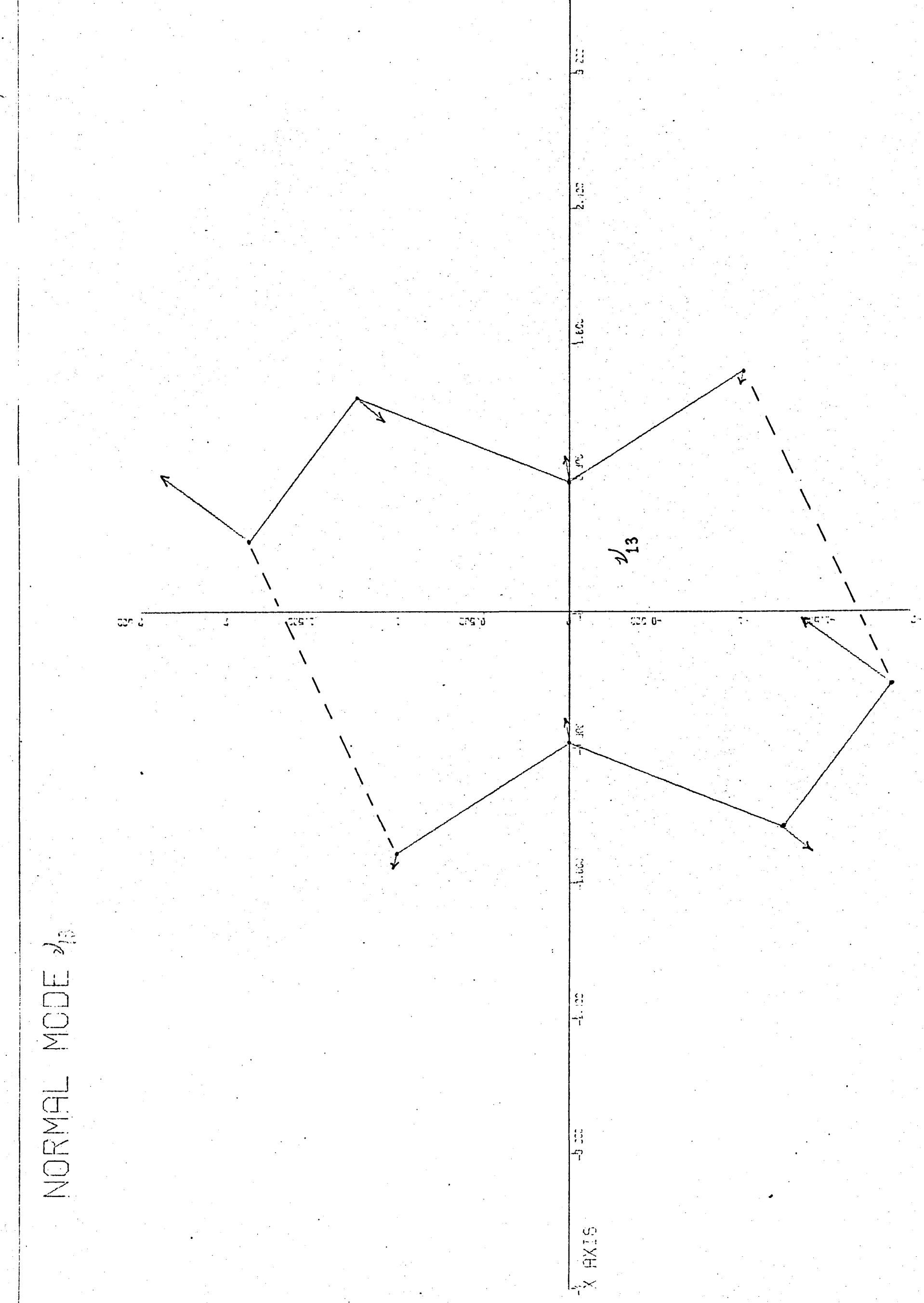


22.6 The expected frequencies of deuterated oxalic acid mononer

If we assume that the goonetry of the deuterated ocalic acid monomer is the same as that for gaseous oxalic acid, the expected frequencies of the deuterated oxalic acid nonomer nay be obtained by solving the secular equation using the sems 6 and E ratrices excopt here the mass of the hydrogen is replaced by that of deuterium.

Using 1st nodel

Tables 33 and 34 show the expocted frequencies and tho potential onergy distribution of deuterated oxalic acid monongr. They were calculated using gaseous oxalic acid force constants for Ist model.

\section{Tabie 33}

The calcuiated frequenc $=3$ oxalic acid mononer

$$
\text { using } 2 \text { st model }
$$

Calculated frequencies

Oxalic acid deuterated oxalic acid

$\begin{array}{rrrr}v_{1} & 831.6 & 750.2 & 81.4 \\ v_{2} & 1849.3 & 1847.5 & 1.8 \\ v_{3} & 1453.6 & 1423.5 & 30.1 \\ v_{4} & 3490.3 & 2537.4 & 952.9 \\ v_{5} & 407.0 & 396.4 & 10.6 \\ v_{6} & 625.3 & 621.7 & 4.1 \\ v_{7} & 1229.0 & 1017.9 & 211.1 \\ v_{8} & 1761.0 & 1758.1 & 2.9 \\ v_{9} & 1334.4 & 1297.2 & 37.2 \\ v_{10} & 3490.1 & 2537.1 & 953.0 \\ v_{11} & 650.4 & 614.5 & 41.9 \\ v_{12} & 305.6 & 295.7 & 9.9 \\ v_{13} & 1228.6 & 983.7 & 244.9\end{array}$

Colculeted shift

$$
H \rightarrow D
$$

1.4

1.8

30.1

10.6

4.1$$
2.9
$$

37.2

41.9

9.9

244.9 

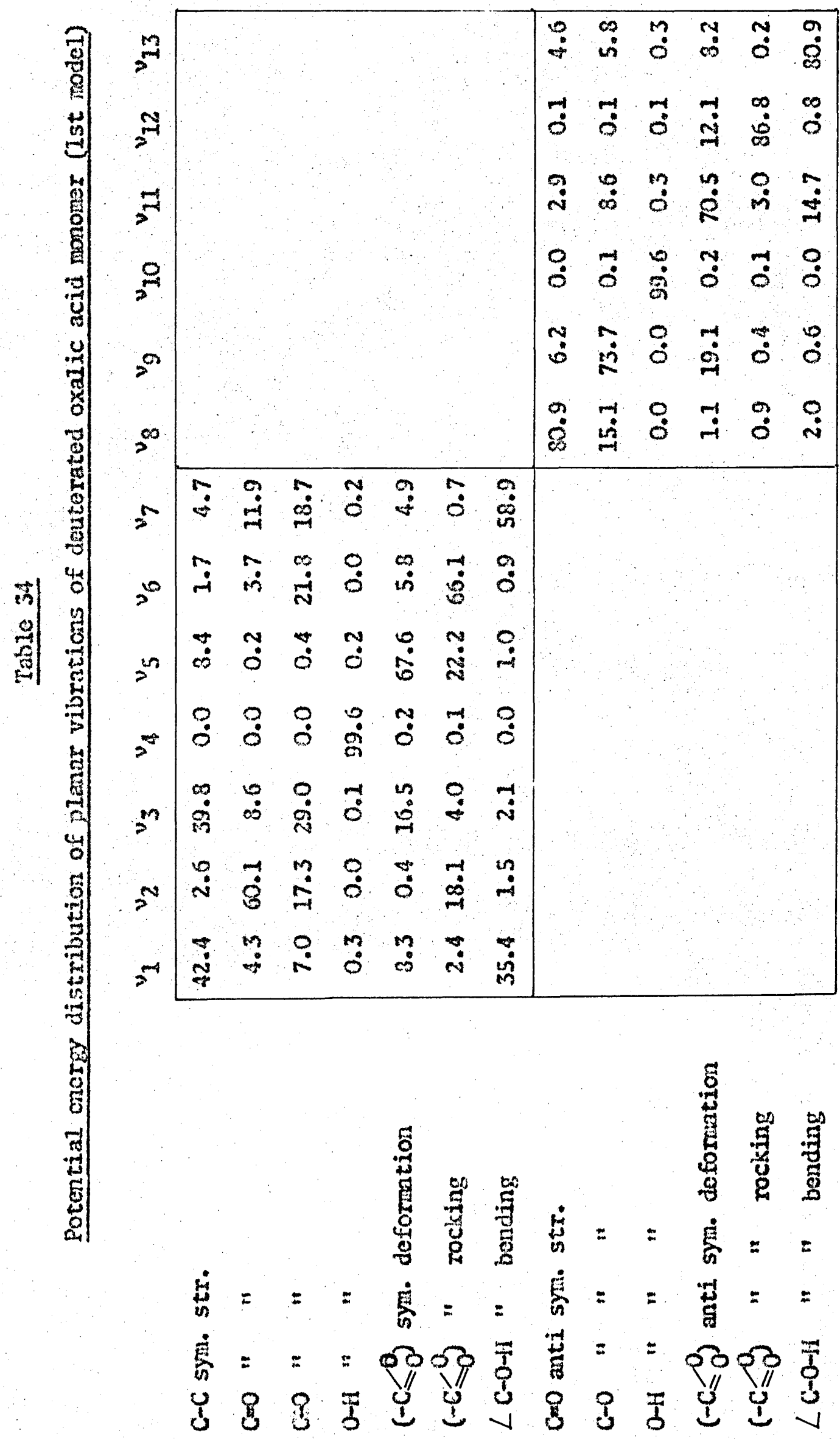
Using Znd modei

The expected frequencies and the potential energy distribution for deuterated oxalic acid wero calculated using the constants fron gascous oxalic acid 2nd nodel. The results are in Tables 35 and 35 .

\section{Table 35}

Calculated frequencies of deuterated oxalje acid nonorar

znd nodel

Calculated frequencies $\left(\mathrm{cm}^{-1}\right)$

oxalic acid deutorated cxalic acid

$v_{1} \quad 820.9$

$v_{2} \quad 1810.9$

$v_{3} \quad 1409.6$

$v_{4} \quad 3471.9$

$v_{5} \quad 8.4$

$v_{6} \quad 414.6$

$v_{7} \quad 1246.2$

$v_{5} \quad 1812.5$

$v_{9} \quad 1237.0$

$v_{10} \quad 3471.9$

$v_{11} \quad 302.0$

$v_{12} \quad 454.9$

$v_{13} \quad 1324.4$
804.5

1810.

1358.7

2523.7

8.3

400.7

937.3

1811.6

1250.2

2523.8

298.9

451.4

933.7 $\frac{\text { Calculated shift }\left(\mathrm{cm}^{-1}\right)}{1+D}$

16.4

0.5

50.9

948.2

0.1

4.9

308.9

0.9

$-13.2$

948.1

3.1

3.5

385.7 


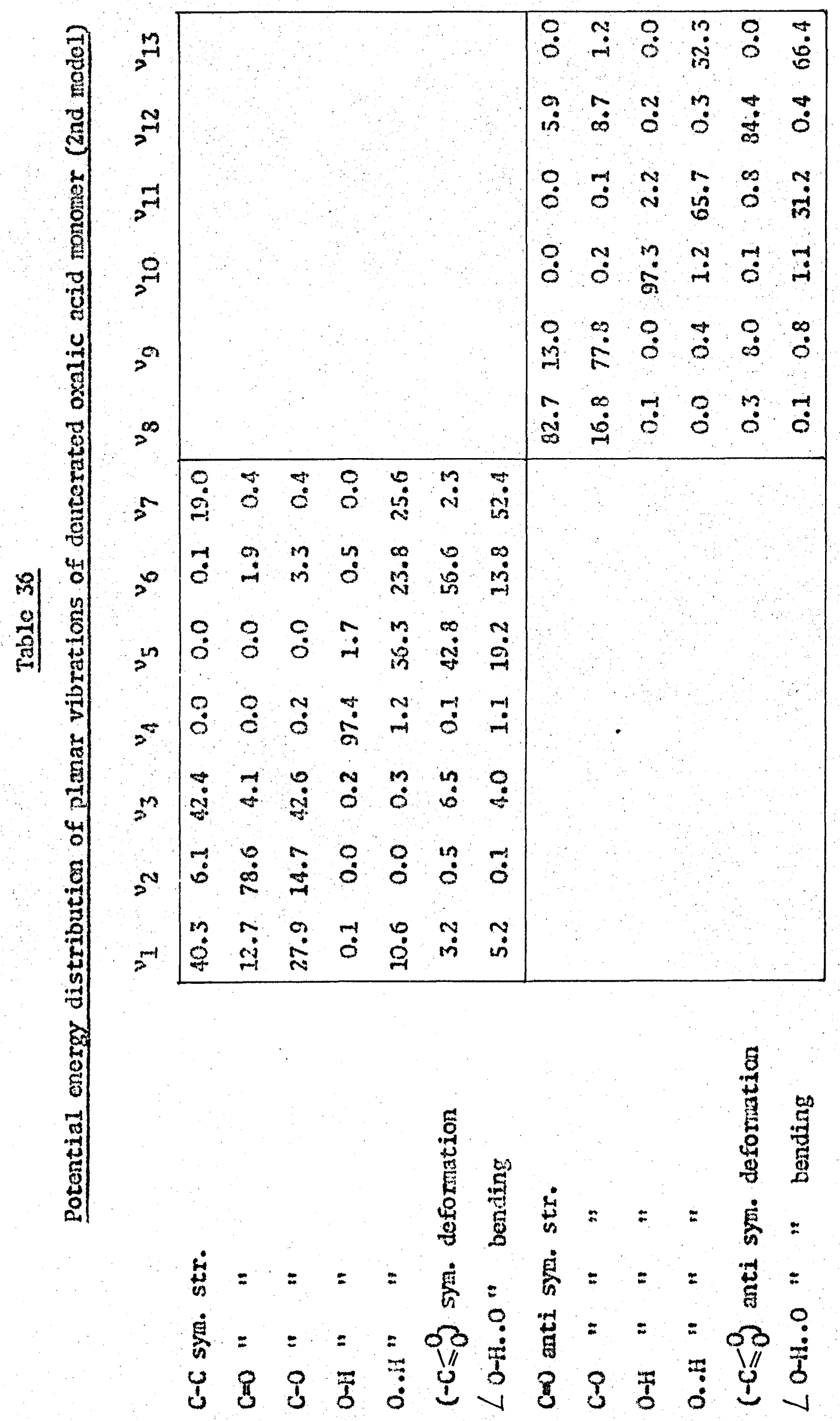




\subsection{The out of plane frequencies}

The expected out of plane frequencies consist of 3 infrared and 2 Ranan active modes. As only 1 or 2 infrared bands could possibly be assigned to out of plane vibrations, and as no out of plane Raman bands were observed, it was considered to be pointless at this stage to make any calculation for the fundamontals. 


\section{CHAPTER 23}

CONCLUSTONS AND SUGGESTIONS FOR FUTURE WORK

Oxalic acid is an asymetric top molecule of $\mathrm{C}_{2 \mathrm{~h}}$ point group. It has 18 nodes of vibration, 13 inplane and 5 out of plame fundarentals as follows:

$$
r=7 \mathrm{Ag}+6 \mathrm{Bu}+2 \mathrm{Bg}+3 \mathrm{Au}
$$

$7 \mathrm{Ag}$ and $6 \mathrm{Bu}$ are inplane vibrational nodes, the others are out of plane.

13 bands of gasoous oxalic acid were observed, 8 in the infrared and 5 in the Raman. 10 were assigned to inplane fundamentals, 2 were assignod to out of plane fundarantals, and only one was assigned to a conbination node.

As a highly asymotric cop nolecule of point group $C_{2 h}$ the infrared bands are expected to have $A, B, C$ and hybrid type envelopes. In the spectra recorded only 2 banks showed $a$ separation of $\mathrm{P}$ and $\mathrm{R}$ branches.

The theoretical band envelopes were studied for the roason that the band assignment could possibly bo made and the out of plane vibrations could be picked out. The attempt to nake the band assignment by comparing the observed band contours of gascous oxalic acid to that of theoretical band contours given by Uedo and Shimanouchi (71) was made and it was found thet

(a) The theorotical band contours for $A$ and $C$ typo bands are usually similar, and thus it is inpossible to identify the out of plane modes. 
(b) Most of the gaseous oxalic band shapes are not similar to the expected band contours. This is because most observed band envolopes are hybrid bands.

Thus it is not possible to pick out the out of plane vibrations. Nor is it possible to -distinguish botween the two models from the observed band contours obtained in this work because the moments of inortia are so sinilar.

Infrared and Raman experinents face different problens. In the infrared the main problens are the condensation of the sample on the $\mathrm{KBr}$ windows and the interference caused by decomposition products. Both problens give a high background, and thus, some gaseous oxalic acid bands may be undetected. Reduction of both problems is achieved by keeping the window temperatures slightly higher than the cell body and by ovacuating the cell continuously.

In the Raman no condensation problen arises and the decomposition products do not interfere so much with the gaseous oxalic acid bands. The gaseous oxalic ecid Raman bands are very distinctive from the others. Most of the Renan bands are strong enough for depolarization measurements. Thus, the assignuent of the Raman bands was made easier than those in the infrared.

However, as the Raman bands are much nore difficult to detect than the infrared, the Ranan experiment needs

(a) very accurate lining up;

(b) a higher tomperature heated coll;

(c) powerful laser and detector. 
The calculations were made using both models of gaseous oxalic acid which were suggested by Nahlouska, Nahlovsky and Strand (78). They published their work on electron diffraction of gaseous oxalic acid during the course of this work.

It was hoped that some useful information might be obtained, and thus the position of the $H$ atoms in this molecule could be distinguished.

Unfortunately, from the results obtained it is difficult to distinguish between the two models for the following reasons:-

(1) The asymotric O-II stretching band is shifted to lower frequancy without band broadening.

(2) The out of plane fundanentals can not be picked out.

(3) Not all the fundamentals ware observed.

(4) The observed values can be fitted quite well to the celculated values for both models.

In order to be able to conplete the work the following future work on

(a) Infrared and Raman spectra of deuterated oxalic acid monomer;

(b) Far infrared for gaseous oxalic acid;

(c) Higher resolution infrared spectra and hybrid profile calculations

is required.

Further inprovement for the infrared experinent can bo made by increasing the pathlength by using a folded path multiple travorsal gas cell and heating to a lower temperature to reduce decomposition. 
1. Steole, D., Theory of Vibrational Spectroscopy, Saunders, London, 1971.

2. Herzberg, G., Infrared and Raran Spectra of Polyatomic Bolecules. Van Nostrand, Now York, 1945.

3. Wilson, E. B., Decius, J. C., and Cross, P. C., Molecular vibrations. MeGrav-liill, New York, 1955.

4. Colthup, N. B., Daly, L. H., and Wiberley, S. E., Introduction to Infrared and Raman Spectroscopy. Acadenic Press, New York, 1964.

5. Davies, M., Infrared Spectroscopy and blecular Structure. Elsovier, London, 1963.

6. Miller, R. G. J., and Stace, B. C., Laboratory Nethods in Infrared Spectroscopy. Heydon and Son, London, 1972.

7. Herzberg, G., Electronic Spectra of Polyatonic lolecules. Van Nostrand, Now York, 1966.

8. Sugden, T. M., and Kenney, C. N., Microvave Spectroscopy of Gases. Van Nostrand, New York, 1965.

9. Bellamy, L. $\mathrm{J}$. The Infrared Spectra of Complex Holecules. Nethuen, London, 1962.

10. Bellany, L. J., Advances in Infrared Group Frequencies. Methuen, London, 1968.

11. Tobin, M. C., Laser Reman Spectroscopy. Wiley Interscience, New York, 1971.

12. Mizushina, S., Structure of Molecules and Internal Rotation. Acadenic Press, New York, 1954. 
13. Nakamoto, $K_{.}$, Infrared Spectra of Inorganic and Co-ordination Compounds. Wiley and Sons, New York, 1963.

14. A1len, H. C., and Cross, P. C., Mblecular Vib-rotors. Wiley and sons, New York, 1963.

15. Hackforth, H. L., Infrared Radiation. McGran-Hill, New York, 1960.

16. Martin, A. 日., Infrared Instrumentation and Techniques. Elsevier, London, 1966.

17. Willians, D. H., and Fleming, I., Spectroscopic Methods in Organic Chemistry. WeGraw-Hi11, New York, 1966.

18. King, G. W., Spectroscopy and holecular Structure. Holt, Rinehart and Winston, How York, 1964.

19. Atkin, R. H., Mathematics and Wave Nochenics. Heinemann, London, 1964.

20. Kinzitt, M. F., Far Infrared Techniques. Pion, London, 1970.

21. Pimentel, G. C., and MeClellan, A. L., The Hydrogen Bond. Freeman, San Francisco, 1960.

22. Hamilton, W. C., and Ibers, J. A., Hydrogen Bonding in Solids. Benjemin, New York, 1968.

23. Margenau, H., and Murphy, G. M., The Mathenatics of Physics and Chenistry. Van Nostrand, New York, 1968.

24. Marn, D. E., Shimanouchi, T., Meal, J. H., and Fano, L., J. Chen. Phys. 27, No. 1, 43-59, 1957.

25. Overend, J., and Schoror, J. R., J. Chem. Phys, 32, No. S, 1289-1303, 1960.

26. Badger, R. M., and Zumsalt, L. R., J. Chem. Phys. 6, 711-717, 1938. 
27. Cross, P. C., Hainer, R. H., and King, G. W., J. Chem. Phys., 12. No. 6, 210-221, 1944.

28. Durig, J. R., and Hanum, S. E., J. Giem. Phys, 54, No. 6, 2367-2374, 1971.

29. Wilson, E. B., J. Chem. Phys. 3, 276-285, 1935.

30. Morino, Y., and Kuchitsu, K., J. Chena. Phys. 20, 1809, 1952.

31. Kakiuti, Y., and Shimanouchi, T., J. Chen. Phys. 25, No. 6. 1252-1254, 1956.

32. Shimanouchi, T., Kakiuti, Y., and Gamo, I., J. Chen. Phys. 25, No. $6,1245-1251,1956$.

33. Miller, F. A., and Crawford, B. L., J. Chen. Phys. 14, No. 4, $282-292,1946$.

34. Wilson, E. B., J. Ciem. Phys. I, 1047-1051, 1939.

35. Costain, C. C., J. Chom. Phys. 29, No. 4, 864-874, 1959.

36. Bratoz, S., and Haázi, D., J. Chen. Phys. 27, No. 5, 991-997, 1957.

37. Collke, R. C., Mills, I. Mo, Person, W. B., and Crawford, B., J. Chem. Phys. 25, No. 6, 1266-1275, 1956.

38. Scherer, J. R., and Overend, J., J. Chen. Phys. 33, No. 6, $1681,1960$.

39. Kishida, S., and Nakamoto, K., J. Chem. Phys. 41, No. 6, $1554-1563,1964$.

40. Millikan, R. C., and Pitzor, K. S., J. Chen. Phys. 27, No. 6, 1305-1308, 1957.

41. Delaplane, R. G., and Ibers, J. A., J. Chem. Phys. 45, 345I, 1966.

42. Laviel, J. L., and Marechal, Y., J. Chem. Phys, 54, No. 3, 1104-1107, 1971.

43. Lemer, R. G., Dailey, B.P., and Friend, J. P., J. Chen. Phys., 26, No. 3, 680-683, 1956. 
44. Wilnshurst, J. K, J. Chan. Phys. 25, No. 6, 1171-1173, 1956.

45. WiImshurst, J. K., J. Chen. Phys. 25, No. 3, 478-480, 1956.

46. Karle, I. L., and Karle, J., J. Chen. Phys. 22, No, 1, $43-45,1954$.

47. Heanan, R. C., J. Chern. Phys. $8,252-258,1940$.

48. Bonner, L. G., and Hofstadler, R., J. Chem. Phys. 6, 531-543, 1938.

49. Miyazawa, T., and Pitzer, K. S., J. Chem. Phys. 30, No. 4, 1076-1086, 1959.

50. Torok, F., and Pulay, P., J. Nol. Struct. 3, 1-9, 1969.

51. Murata, H., and Kawai, K., J. Chem. Phys 25, 589, 1956.

52. Scherer, J. R., Spectrochin. Acta. 24A, 747-770, 1968.

53. Alti, G., Calasso, V., and Costa, G., Spectrocinin. Acta. 21, $649-658,1965$.

54. Yamaguchi, A., Ichishime, I., and Mizushina, S., Spectrochin. Acta. 12, 295-298, 1958.

55. Seth-Paul, W. A., and Dijkstra, G., Spectrochim. Acta. 23A, 2851-2870, 1967.

56. Hollas, J. H., Spectrochin. Acta. 22, 81-95, 1956.

57. Susi, Ho, and Scherer, J. R., Spectrochin. Acta. 25A, 1243$1263,1969$.

58. Blumenfeld, S. M., and Fast, H., Spectrachin. Acta 24A, 1449-1459, 1968.

59. Carlson, G. L., Witkowski, R. E., and Fatelcy, W. G., Spectrochim. Acta 22, 1117-1123, 1966.

60. Susi, H., and Zell, T., Spectrocinim. Acta 19, 1933-1945, 1963. 
61. Evans, J. C., and Overend, J., Spectrochim. Acta 19 , $701-704,1963$.

62. Bellany, L. J., and Pace, R. J., Spectrochim. Acta 19, $435-442,1963$.

63. Jakobsen, R. J., Makawa, Y., and Brasch, J. H., Spoctrochim. Acta 23A, 2199-2209, 1967.

64. Buteler, M. J. and kckean, D. C., Spectrochins Acta 21, $465,1965$.

65. Flotcher, H. H. and Thompson, W. T., J. Hol. Spec. 25, $240-268,1968$.

66. Cold, Ro, Dowling, J. M., and Moister, A. G., J. Mbl. Spec. 2. $9-26,1958$.

67. Begun, G. Mo and Fletcier, W. H., J. Ko1. Spec. 4, 388-397, 1960.

68. Mills, I. M., J. 161. Spec. 5, 334-340, 1960.

69. Parkin, J. E., J. MbI. Spec. 15, 483-501, 1965.

70. Tominson, G. E., Cumutte, B., and hathaway, C. E., J. No1. Spec. $36,26-33,1970$.

71. Ueda, T. and Shimanouchi, T., J. Mo1. Spec. 28, 350-372, 1968.

72. Jones, R. L., J. Mol. Spec. 11, 411-421, 1963.

$$
\begin{aligned}
& \| \quad " \quad \quad \quad \quad \quad \quad, 460-463,1961 . \\
& \|\quad\| \quad 2,581-586,1958 .
\end{aligned}
$$

73. Kirk-0ciner Encycl. Chen. Techno1., 2nd ed. 14, 356-373, 1967 (Eng.).

74. Strand, T. G., Acta Chem. Scand. 21, 2111-2118, 1967.

75. Dah1, T. and Hasse1, O., Acta Chem. Scand. 22, 2851-2866, 1968.

76. Cyvin, S. J., Alfhoin, I., and Hagen, G., Acta Chem. Scand. 24, $3038-3042,1970$.

77. Altheim, I., and Cyvin, S. J., Acta Chem. Scand. 24, 3043-3049, 1970. 
78. Nahlovska, Z., Nahlovshy, B., and Serand, T. G., Acta Chen. Scand. 26(7), 2617-2628, 1970.

79. Grvin, S. J. and Althein, I., Acta Chon. Scand. 24(7), 2648-2650, 1970.

80. Parrett, F. W., Record of Chenical Progress 30(2), 135-146, 1969.

81. Shinanouchi, T., Pure App1. Chen. Z(1), 131-145, 1963.

82. Stepanov, A. V. and Pazukhin, E. M., J. Inorg. Crem. 15(6), $761,1970$.

83. Coulson, C. A. and Colebiowski, A., J. Chem. Soc., 4948-4952, 1960.

84. Hadzi, D. and Kobilerov, N., J. Cheza. Soc. A, 430-445, 1966.

85. Hadzi, D., J. Chom. Soc. 5128-5138, 1962.

86. Cox, E. G., Dougi11, M. W., and Jeffrey, G. A., J. Chem. Soc. 4854, 1952.

87. Bradloy, R. S. and Cotson, S., J. Chem. Soc. 1684-1689, 1953.

88. Dolaplane, R. G. and Ibers, J. A., Acta Cryst. B25, 2425, 1969.

89. Iwasaki, F. F. and Saito, Y., Acta Cryst. 23, 56, 1967.

90. Iwasaki, F. F., Iwasaki, H., and Saito, Y., Acta Cryst. 23, $64,1967$.

91. Almal, F. R. and Cruickshank, D. H. J., Acte Cryst. 6. 385, 1953.

92. Coppeas, P. and Sabine, T. M., Acta Cryst. B25, 2442, 1969.

93. Sabine, T. M. and Cox, G. W., Acta Cryst. B25, 2437, 1969.

94. Nakanura, J. X. and Schwarz, S. E., Appliod Optics Z(6), 1073-1078, 1968.

95. Tobin, M. C., Appliod Optics $9(2), 502-503,1970$.

36. Morton, G. A., Applied optics Z(1), 1-10, 1968.

97. Brools, W. V. Fo and Haas, C. M., J. Phys. Chem. 71 (3), 650-655, 1967. 
98. Pava, B. M. and Stafford, E. E., J. Phys. Cren. $\underline{72}$ (13), $4628-4630,1968$.

99. Saunders, J. E., Bentley, F. F. and Katon, J. E., Appl. Spoc. $22(4), 286-294,1968$.

100. Rendall, H. M., Demnison, D. M., Ginsburg, N., and Weber, L. R., Phys. Rov. 52, 160-174, 1937.

101. Urey, H. C. and Bradloy, C. A., Phys. Pev, 38, 1969-1978, 1931.

102. Wang, S. C., Phys, Rev. 34, 243-252, 1929.

103. Gerhard, S. L. and Dennison, D. H., Phys. Rev. 43, 197-204, 1933.

104. Shibate, S. and Kimura, 11., Bul1. Cron. Soc. Japan 27, $485,1954$.

105. Durig, J. R. and Hannum, S. B., J. Cryst. Bol. Struct. 1, 131-137, 1971.

106. Peldus, J. and lirabo, P., Theort. Chin. Acta 11, 401-410, 1968.

107. Heuric, Ko and lovak, A., C. R. Aced. Sc. Paris Ser, B, 694-697, 1957.

108. Bardet, L. and Fleiry, M. G., C. R. Acad. Sc. Paris Ser. B, $983-985,1967$.

109. Bardet, L., Meury, M. G. and Tabacik, V., C. R. Acad. Sc. Paris Ser. B, 1277-1280, 1970.

110. Lorenze11i, M. V. and Alemagna, A., C. R. Acad. Sc. Paris $3626-3528,1963$.

11z. Varsányi, Gy., Sz"oke, S., and Farago', T., Acta Chim. Acad. Sci. Hungary 34, 411, 1962.

112. Varsányi, Gy., Acta Cnin. Acad. Sci. Hungary 25, 255, 1960.

113. Clagus, D. and Novai, A., J. Mo1. Struct. 5 , 149-152, 1970. 
114. Miyazas, T. and pitzer, K. S., J. Aa. Chen. Soc. 81, $74,1959$.

115. Alfano, R. H. and Ociman, N., J. Opt. Soc. Amer. S8(1), 90-05, 1958.

116. Kraitchmen, J., Nator. J. Phys. 21, 17-24, 1953.

117. Vonkateswarlu, R., Marfam, 5, and Girijavallabhan, C. P., Bur1. Soc. Roy. Se1. Latge, 9-10, 576-582, 1967.

118. Butenfold, S. M., leddy, S. P. and Wo1sh, L. P., Canad. J. Phys. $48,513-520,1970$.

119. Staco, B. C., perscanl corminication.

120. Rey, B. S., Z. Physik 76, 74, 1932.

221. Fing, G. W., habuer, R. H. and Cross, P. C., J. C. P. II, $27,1943$.

122. Pleezek, Go and Teller, E., Z. Physik E1, 209, 1933.

123. Aldous, J. and 澈11s, I. $H_{*}$, Spectrochs. Aeta 18, 1075-1091, 1052.

124. Marrignan, M.R. and Bardet, M. L., C. R. Acad, Sci. Paris t235, 1022, 1951 .

125. Seth-paul, H. A. and atseyer, H., J. No1. Struct. 3, 11-20, 1959.

126. Seth-Paul, W. A., J. Wol. Struct. 3, 405-427, 1969.

127. Dieke, G. H. and Xistiakowsky, G. B., Phys. Rov. 45, 4, 1934.

128. Welt, D., Infrared Vapour Spectra. Hoyden mal son, London, 1970.

129. Piorson, R. \#., Metchor, A. N., and Gantz, E. St. C., Analyt. Chom. 28, No. 8, 1218-1239, 1955.

130. Loador, J., Basic Laser karinn Spectroseopy. Hoyken and son, Londor, 1970. 